\title{
Sport Sciences for HealTh
}

Founded by the School of Exercise Sciences, University of Milan

Official Journal of the Italian Society of Exercise and Sport Sciences

\section{Editor-in-Chief}

L. Luzi, Milano

\section{Co-Editor}

A. Veicsteinas, Milano

\section{Associate Editor}

F. Esposito, Milano

\section{Advisory Board}

A. Concu, Cagliari

G. Fanò-Illic, Chieti

G. Ferretti, Brescia

G. D’Antona, Pavia

P. DeFeo, Perugia

A. Deligiannis, Thessaloniki

A. Lenzi, Roma

M.V. Narici, Manchester

M. Olfert, Morgantown, West Virginia

RS. Richardson, Salt Lake City, Utah

F. Schena, Verona

G.I. Shulman, New Haven, Connecticut

\section{黛 Springer}




\section{SpORT SCIENCES FOR HEALTH}

\section{Alims and scope}

The journal Sport Sciences for Health publishes reports of experimental and clinical research on the physiology and pathophysiology of physical exercise, with special focus on the mechanisms through which exercise can prevent or treat chronic-degenerative disease, contributing to health maintainance in the population. The journal welcomes original contributions on biochemical, physiological pathophysiological and clinical aspects of physical exercise in the prevention of diabetes mellitus, insulin resistance syndrome, cardiovascular diseases, inflammation and some forms of cancer, as well as reports of studies identifying the "dose" of physical exercise suitable for prevention and that suitable for treatment of specific diseases, for the different sports activities. A special section of the journal is the Pharmacoeconomics Forum, which publishes contributions on the effects of prevention and exercise programs on health maintainance costs. Reports are published in the form of origina articles, short communications and letters to the Editor; invited reviews and editorials are also published. Finally, workshop reports are also welcome in Sport Sciences for Health.

Manuscripts submitted for publication must contain a statement to the effect that all human studies have been reviewed by the appropriate ethics committee and have therefore been performed in accordance with the ethical standards laid down in an appropriate version of the 1964 Declaration of Helsinki. It should also be stated clearly in the tex that all persons gave their informed consent prior to their inclusion in the study. Details that might disclose the identity of the subjects under study should be omitted. Reports of animal experiments must state that the "Principles of laboratory animal care" (NIH publication No. 86-23, revised 1985) were followed, as well as specific national laws (e.g. the current version of the German Law on the Protection of Animals) where applicable. The Editor-in-chief reserves the right to reject manuscripts that do not comply with the abovementioned requirements. The author will be held responsible for false statements or for failure to fulfil the above-mentioned requirements.

The journal is open for publication of supplements and for publishing abstracts of scientific meetings. Condition can be obtained from the Editor-in Chief or the publisher.

\section{Copyright}

Submission of a manuscript implies: that the work described has not been published before (except in form of an abstract or as part of a published lecture, review, or thesis); that it is not under consideration for publication elsewhere; that its publication has been approved by all coauthors, if any, as well as - tacitly or explicitly - by the responsible authorities at the institution where the work has been carried out. The publisher will not be held legally responsible should there be any claims for compensation. Transfer of copyright to the publisher becomes effective if and when the article is accepted for publication. The copyright covers the exclusive right (for U.S. Government employees: to the extent transferable) to reproduce and distribute the article, including reprints, translations, photographic reproductions, microform, electronic form (offline, online) or other reproductions of similar nature.

All articles published in this journal are protected by copyright, which covers the exclusive rights to reproduce and distribute the article (e.g., as offprints), as well as all translation rights. No material published in this journal may be reproduced photographically or stored on microfilm, in electronic data bases, video disks, etc., without first obtaining written permission from the publisher. The use of general descriptive names, trade names, trademarks, etc., in this publication, even if not specifically identified, does not imply that these names are not protected by the relevant laws and regulations.

An author may self-archive an author-created version of his/her article on his/her own website and his/her institution's repository, including his/her final version: however his/she may not use the publisher's PDF version which is posted in www.springerlink.com. Furthermore, the author may only post his/her version provided acknowledgement is given to the original source of publication and a link is inserted to the published article in Springer website. The link must be accompanied by the following text: "The original publication is available at http://www.springerlink.com". Please use the appropriate URL and/or DOI for the arti cle. Articles disseminated via SpringerLink are indexed, abstracted and referenced by many abstracting and information services, bibliographic networks, subscription agencies, library, networks, and consortia.

While the advice and information in this journal is believed to be true and accurate at the date of its publication, neither the authors, the editors, nor the publisher can accept any legal responsibility for any errors or omissions that may be made. The publisher makes no warranty, expressed or implied, with respect to the material contained herein.

Special regulations for photocopies in the USA. Photocopies may be made for personal or in-house use beyond the limitations stipulated under Section 107 or 108 of U.S. Copyright Law, provided a fee is paid. All fees should be paid to the Copyright Clearance Center, Inc., 21 Congress Street, Salem, MA 01970, USA, stating the ISSN 1824-7490, the volume, and the first and last page numbers of each article copied. The copyright owner's consent does not include copying for general distribution, promotion, new works, or resale. In these cases, specific written permission must first be obtained from the publisher.

The Canada Institute for Scientific and Technical Information (CISTI) provides a comprehensive, world-wide document delivery service for all Springer journals. For more information, or to place an order for a copyright-cleared Springer document, please contact Client Assistant, Document Delivery, CISTI, Ottawa K1A 0S2, Canada (Tel.: +1-613-9939251; Fax: +1-613-9528243; e-mail: cisti.docdel@nrc.ca).

3 Subscription information

ISSN 1824-7490 (print version)

ISSN 1825-1234 (electronic version)

\section{Subscription rates}

For information on subscription rates please contact: Customer Service

The Americas (North, South, Central America and the Caribbean)

journal-ny@springer.com

Outside the Americas

subscriptions@springer.com

Orders and inquiries

The Americas (North, South, Central America and the Caribbean)

Springer Journal Fulfillment

P.O. Box 2485

Secaucus, NJ 07096-2485, USA
Tel. 800-SPRINGER (777-4643)

Tel. +1-201-348-4033 (outside US and Canada) Fax +1-201-348-4505

e-mail: journals-ny@springer.com

Outside the Americas

via a bookseller or

Springer Distribution Center $\mathrm{GmbH}$

Haberstrasse 7, 69126 Heidelberg, Germany

Tel. +49-6221-345-4304

Tel. +49-6221-345-4304

Fax +49-6221-345-4229

e-mail: subscriptions@ springer.com

Business hours: Monday to Friday

8 a.m. to 8 p.m. local time and on German public holidays

Cancellations must be received by September 30 to take effect at the end of the same year.

Changes of address. Allow six weeks for all changes to become effective. All communications should include both old and new addresses (with postal codes) and should be accompanied by a mailing label from a recent issue. According to $\S 4$ Sect. 3 of the German Postal Services Data Protection Regulations, if a subscriber's address changes, the German Post Office can inform the publisher of the new address even if the subscriber has not submitted a formal application for mail to be forwarded. Subscribers not in agreement with this procedure may send a written complaint to Customer Service Journals, within 14 days of publication of this issue.

Back volumes. Prices are available on request.

Microform editions are available from: ProQuest. Further information available at: http://www.il.proquest.com/umi/

4 Electronic edition

An electronic edition of this journal is available at springerlink.com.

Managing director

Antonella Cerri, Milano, Italy

Production editor

Paola Gregori

Springer-Verlag Italia Srl

Journal Department

Via Decembrio 28, I-20137 Milano, Italy

Tel.: +39-02-54259770

Fax: +39-02-54259701

e-mail: paola.gregori@springer.com

Address for courier, express and registered mail: Springer-Verlag Italia Srl

Via Decembrio 28, I-20137 Milano, Italy

Responsible for advertisements

Carlotta d'Imporzano

Springer-Verlag Italia Srl

Via Decembrio 28, I-20137 Milano, Italy

Tel.: +39-02-54259704

e-mail: carlotta.dimporzano@springer.com

Typesetter

Ikona S.r.1., Milano, Italy

Printer

Geca SpA

Cesano Boscone (MI)

Printed on acid-free paper

Registrazione del Tribunale di Milano

Distribution in Italy occurs according to the Italian Law 196/2003

Springer

is a part of

Springer Science+Business Media

springer.com

(C) Springer-Verlag Italia 2012

Via Decembrio 28, I-20137, Milano, Italy

Printed in Italy 


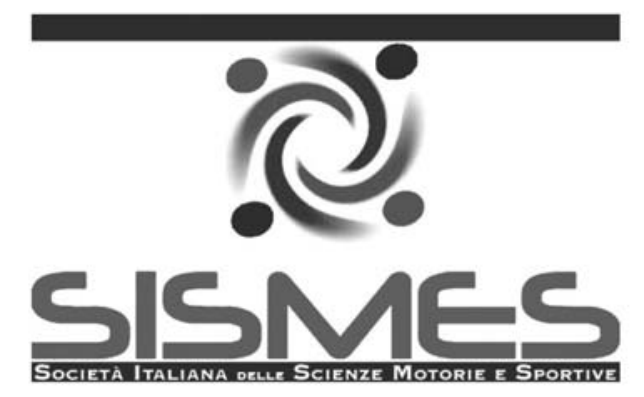

\section{National Congress}

Palermo

5 - 7 October 2012

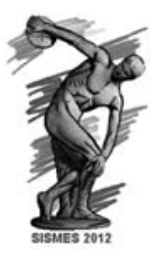

\section{ABSTRACT BOOK}

Congress President

FEDERICO SCHENA

Scientific Committee

ANTONIO PALMA (Chair)

LAURA CAPRANICA

MARCELLO FAINA

CLAUDIO ROBAZZA

ARSENIO VEICSTEINAS

LINA BUONO

MARCELLO TRAINA

Local Organizing Committee

GIUSEPPE ALAMIA

NATALE SALVO
Scientific Secretary

ANTONINO BIANCO

GIUSEPPE BATTAGLIA

MARIANNA BELLAFIORE
Organizing Secretary

ANNA RITA GULLI

MARGHERITA RACCUGLIA

SALVATORE PALAZZOLO 


\section{Springer}

\section{springer.com}

\section{Books Highlights in Sport Medicine}

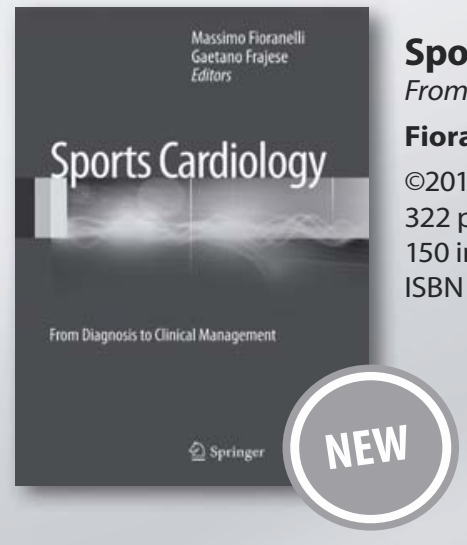

\section{Sports Cardiology}

From Diagnosis to Clinical Management

Fioranelli, Massimo; Frajese, Gaetano (Eds.)

() 2012, Hardcover

322 p 200 illus.,

150 in color.

ISBN 978-88-470-2774-9

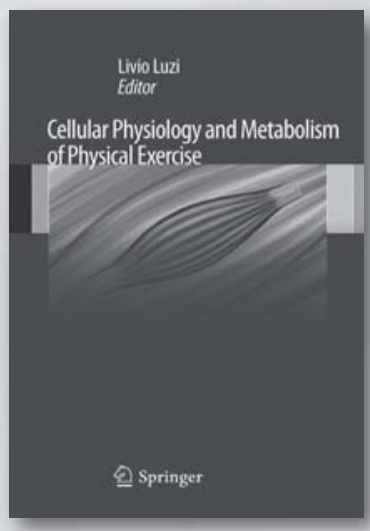

Cellular Physiology and Metabolism of Physical Exercise Luzi, Livio (Ed.)

(c) 2012, Hardcover

$X X, 180$ p. 50 illus. in color.

ISBN 978-88-470-2417-5

\section{Functional Neuroimaging in}

\section{Exercise and Sport Sciences}

Henning Boecker. Charles H. Hillman tukas Scheef - Heiko K. Struder Editors

Functional

Neuroimaging in

Exercise and Sport

Sciences
Boecker, H.; Hillman, C.H.; Scheef, L.;

Strüder, H.K. (Eds.)

(C)2012, Hardcover

XIX, 520 p. 105 illus., 75 in color.

ISBN 978-1-4614-3292-0

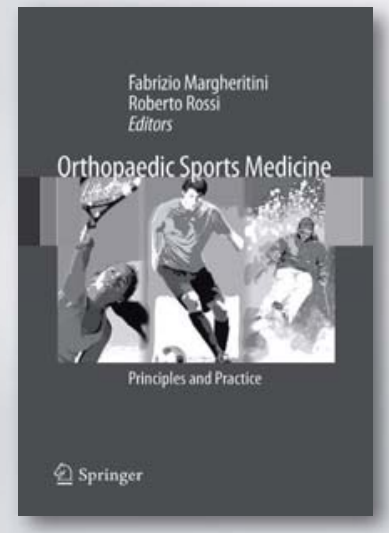

Orthopedic Sports Medicine Principles and Practice

Margheritini, Fabrizio; Rossi, Roberto (Eds.) (c) 2011, Softcover 376 p. 210 illus. ISBN 978-88-470-1701-6 


\section{ORAL PRESENTATIONS}

\section{Friday, October $5^{\text {th }}, 2012$}

\section{Session 1. Motor Learning and Development}

\section{Motor development and physical self-perception of overweight and obese children. Assessment of motor intervention}

\author{
D. Colella ${ }^{1}$, M. Morano $^{1}$, I. Rutigliano ${ }^{2}$, A. Campanozzi $^{2}$
}

${ }^{1}$ Department of clinical and experimental medicine, University of Foggia, Italy

${ }^{2}$ Department of medical and surgical science

Introduction: The motor activities contribute to the child's educational process 1 , promote the prevention of many diseases 2 and promote the development of self-efficacy perceived 3. In the Apulia region to $39,2 \%$ of children of 8 years has excess weight (2010) 4 ; targeted interventions are needed to counteract the sedentary habits5; reduce body weight and increase the self-perception, through a wide repertoire of motor activities.

Methods: It has been carried out an intervention of extracurricular physical activities for 6 months. Motor test have been proposed (T0 vs T1)6: standing long jump; 20 -m speed; $1 \mathrm{Kg}$ medicine ball throw; the self-report: Perceived Physical Ability Scale for Children (PPAS_C)7 to 28 children divided into overweight and obese according to cutt off of Cole8, attending primary school $(\mathrm{F}=12$; age: $\mathrm{M} \pm \mathrm{ds}=9.76 \pm 0.84$; BMI: $\mathrm{M} \pm \mathrm{ds}=24,28 \pm 2,13 ; \mathrm{M}=16$; age: $\mathrm{M} \pm \mathrm{ds}=9.20 \pm 1.54$; $\mathrm{BMI}$

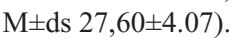

Results: Apart from the descriptive statistics, it was carried out the ANOVA 2 analysis of the variance (test: T0 vs T1) x 2 (sex) repeating the measurements for the last factor, in order to highlight the significant differences related to the dependent variables taken into consideration.

The significativity index was set to $p<0,05$. Some differences emerged with respect to the test-retest within the tests: standing long jump (Female: $\mathrm{p}=0,003$; Male, $\mathrm{p}=0,001$ ); $1 \mathrm{Kg}$ medicine-ball throw (Female: $\mathrm{p}=0,02$; Male: $\mathrm{p}=0,003$ ); in the scale of PPAS_C (Female: $\mathrm{p}=0,000$; Male: $\mathrm{p}=0.04$ ).

We highlight gender differences in the standing long jump: females have higher performance than males ( $p=0,02)$; in the scale of PPAS_C not show differences.

Males and females show a reduction in BMI (T0 vs T1 ): F: $\mathrm{M} \pm \mathrm{ds}=$ $24,28 \pm 2,13$ vs $23,39 \pm 2,03$; M: $27,60 \pm 4,07$ vs $27,38 \pm 4,18$.

Conclusion: The study found positive effects on levels of motor performance and physical self-perception after the program extracurricular. The experiences of success, measured in terms of increased motor abilities of real and perceived, are essential to prevent childhood overweight. Recent studies have shown that physical activity interventions, combined with healthy eating habits, at school and in extracurricular, can reduce children's BMI9.

References

1. Bailey R (2006), JSH,76, 397-401

2. Brambilla $P$ et al. (2010), Int J Obes , 35,16-28

3. Feltz DL et al (2008), Champaign, IL: HK

4. http://www.epicentro.iss.it/territorio/puglia.asp

5. Biddle SJH et al. (2011), Br J Sports Med, 45, 937-942

6. Morrow JR et al. (2005), Champaign, IL: HK

7. Colella D et al. (2008), SBP, 36,841-8

8. Cole TJ et al. (2000), BMJ, 320,1240-3

9. Lavelle HV et al.(2012), J Public Health, 20,1-10
The psychobiosocial state inventory: preliminary evidence of factorial validity

\section{Bortoli, M. Bertollo, C. Robazza}

Ehavioral Imaging and Neural Dynamics (BIND) Center Dept Medicine and Aging Sciences, D'Annunzio University, Chieti, Italy

Aim: The purpose was to provide preliminary evidence of the factorial validity of a new measure intended to assess psychobiosocial (PBS) states in sport. PBS states are situational, multimodal, and dynamic manifestation of total human functioning that include eight interrelated modalities: cognitive, affective, motivational, volitional, bodily, motor-behavioural, operational, and communicative (Hanin, 2010). Earlier PBS inventory versions were administered in a few studies (e.g., Bortoli \& Robazza, 2007).

Methods: Participants were 183 youths ( 80 girls and 103 boys) aged 13-16 years $(\mathrm{M}=14.8, \mathrm{SD}=0.8)$ involved in individual or team sports. Assessment was conducted in nearby training facilities before a regular practice session. The PBS inventory consists of a 20 -item list of functional descriptors (10 items) and dysfunctional descriptors (10 items) to assess PBS states in the context of sport. An item is composed of three, four, or five descriptors to convey a straightforward representation of an emotional experience. Each item is rated on a five-point intensity scale, ranging from $0=$ "not at all" to $4=$ "very, very much", thinking of "how you usually feel prior to an important competition". Results: Variables were screened for missing values, distribution, and potential outliers. The factorial validity of the inventory (i.e., 10 functional and 10 dysfunctional items included in pleasant and unpleasant dimensions) was examined through confirmatory factor analysis using AMOS 20. Maximum likelihood estimation was employed to estimate the model. Inspection of the Lagrange modification indices suggested model modification with the addition of freely estimated parameters to improve the model fit. The errors of two items of the functional subscale of the PBS descriptors and two items of the dysfunctional subscale were left to covary because they appear to convey the same feeling state. The hypothesized model fitted the data, $2(165)=449.99$, $2 / \mathrm{df}=2.73, \mathrm{GFI}=0.90, \mathrm{CFI}=0.93 ; \mathrm{RMR}=0.04, \mathrm{RMSEA}=0.07$ (0.06-0.08).

Conclusions: Findings of this study suggest that the PBS inventory can be used to assess the multimodal experience of global human functioning, whereby emotional reactions to sport competition are a central component of affective and non-affective experiences.

References

- Bortoli, L., \& Robazza, C. (2007). Dispositional goal orientations, motivational climate, and psychobiosocial states in physical education. In L. A. Chiang (Ed.), Motivation of exercise and physical activity (pp. 119-133). New York: Nova Science Publishers.

- Hanin, Y. L. (2010). Coping with anxiety in sport. In A. Nicholls (Ed.), Coping in sport: Theory, methods, and related constructs (pp. 159-175). New York: Nova Science Inc.

Physical activity levels and motor performance in the middle school

G. Semprini ${ }^{1}$, R. Di Michele ${ }^{1,2}$, F. Merni ${ }^{1,2}$

${ }^{1}$ Facoltà di Scienze Motorie, Università di Bologna, Italia

${ }^{2}$ Dipartimento di Istologia, Embriologia e Biologia Applicata,

Università di Bologna, Italia

Previous studies highlighted the importance of performing physical 
activities in the youth age, showing a relationship between the amount of motor activity performed within a time period and health level. The aim of this study was to relate the quantity of physical activity performed in sports clubs or in the free time with the performance in conditional and coordinative tests in middle school students. 424 students (age: 11-14 years; M:201, F:220) attending a middle school in Bologna performed a survey concerning the time devoted to sports and motor activities, and were classified into three categories, namely low (LML), medium (MML) and high (HML) level of activity. Then, the students performed five conditional and coordinative motor tests: standing long jump, ball throwing from a seated position, launched 10-m running, balance test on a quadrangular basis, and comma test to assess the frequency of manual movements. A three-way analysis of variance was carried out to asse! ss the differences between genders, age groups, and activity levels. Significant differences between genders and age classes were noticed for all the tests. Concerning the physical activity levels, significant differences among all the levels were noticed in the running test, with mean values of $1.69 \pm 0.2 \mathrm{~s}$ (LML), $1.64 \pm 0.2 \mathrm{~s}$ (MML), and $1.58 \pm 0.2 \mathrm{~s}(\mathrm{HML})$. In the standing long jump, there were significant differences between the low activity group $(153 \pm 25 \mathrm{~cm})$ and the other two groups (MML: $163 \pm 28 \mathrm{~cm}$; HML: $168 \pm 24$ ). In the ball throwing test, a significant difference was found between the LHL $(531 \pm 108 \mathrm{~cm})$ and the HML $(534 \pm 112 \mathrm{~cm})$ groups. No differences were noticed concerning the coordinative tests. In conclusion, the differences between groups observed for the running test may be explained considering that this kind of skill is frequently required in many sports disciplines and in free-time activities. The d! ifferences between the LML and the other groups in the standing long $\mathrm{j}$ ump may be attributed to the frequent use of the lower limbs in sports and extrasport activities. Concerning the upper limbs strength, the differences noticed only between the LML and HML subjects may be explained considering that the upper limbs are highly used, but in a less marked way by sportsmen. In coordinative tests, the absence of actual differences between groups may be explained by the fact that such capacities (balance, frequency and accuracy of movements) are often neglected in many sports disciplines and in free-time activities. In fact, these capacities are routinely trained only in some sports as gymnastics and roller skating.

Effect of an ecological school-based physical activity programme on different factors of health and fitness in children aged 9-12 years

\section{Moisio, M. Lanza, F. Schena}

Dip. Scienze Neurologiche, Neuropsicologiche, Morfologiche e Motorie, Università di Verona, Italia.

School can be a powerful influence on promoting youth health but few studies investigated the impact of an "ecological school-based program" for promotion of health-related physical capacities and behavior. The majority of research investigated only selected aspects like body composition or fitness (A Gentile, 2009) or interventions targeted only to children while, in this field, the "ecological approach" include interventions with children, family, schools and, hopefully, community. This study aimed to assess the effect of a 6-month physical activity programme on different factors of health and fitness in children: anthropometric parameters, physical fitness level, physical abilities and psychological aspects.

Methods: One hundred eighty-nine children aged 9-12 years were randomly assigned to Intervention Group (IG - 107 children) or Control Group (CG - 82 children). The programme included information meetings for scholastic headmasters and parents, two physical activity ses- sions per week in addiction to standard physical education, three education meetings about lifestyle for children.

Before and after six months of intervention, anthropometric parameters (BMI, skinfolds, waist and hip circumferences), physical fitness performance (balance, flexibility, arms-legs-abdominal strength, endurance), were assessed. In addition, the following questionnaires were administered: Physical Activity Questionnaire for older children (PAQC), Physical Activity Stage of Change (PASCQ), Perceived Physical ability Scale (PPAS), Perceived Physical Activity Enjoyment Scale (PACES).

Two way ANOVA with repeated measures was used to compare prepost and IG-CG data; one way ANOVA was used to compare pre-post differences between IG and CG.

Results: After the programme, IG shows positive changes in all anthropometric parameters: BMI $(1.5 \% ; \mathrm{p}<0.001)$, waist circumference $(1.9 \% \mathrm{p}<0.05)$, waist-hip ratio $(2 \% \mathrm{p}<0.001)$, Sum of skinfolds $(5.5 \%$; $\mathrm{p}<0.05)$.

The fitness parameters were significantly improved in IG: Balance $(31 \% \mathrm{p}<0.05)$, flexibility $(5.2 \% \mathrm{p}<0.05)$, leg strength $(6.8 \% \mathrm{p}<0.05)$; abdominal strength $(25 \% \mathrm{p}<0.05)$; endurance $(9.7 \% \mathrm{p}<0.05)$; despite the arm strength increase in both group, the modification of IG is significantly higher $(10.3 \% \mathrm{p}<0.05)$.

Finally, also psychological and behaviour factors increased in IG: the PACES $(6.23 \%$; $<0.001)$, PPAS $(9.4 \% \mathrm{p}<0.05)$ and PAQ-C $(23.5 \%$ $\mathrm{p}<0.05$ ). The physical activity stage of change (PASCQ) increased in both groups but in higher values in IG $(66.8 \% \mathrm{p}<0.05)$.

Conclusions: Data confirm that an ecological school-based programme has able to induce positive changes in anthropometric characteristics, quality of lifestyle, physical fitness performance and psychological aspects contributing to increase health in schoolchildren.

\section{A priori evaluation in physical activity promotion. A Delphi tecnique to design a workbook for primary school children}

\section{Rodorigo ${ }^{1}$, M. Scatigna ${ }^{1}$, M. R. Di Gregorio ${ }^{2}$, A. Di Giuliol, M. G. Vinciguerra ${ }^{1}$}

${ }^{1}$ University of L'Aquila, Italy

${ }^{2}$ University of Chieti-Pescara, Italy

Last decades a huge amount of scientific literature showed a negative trend of motor capacities and a parallel increasing burden of risk factors in young people, first of all overweight and its consequences. That arises out from lack of Physical Activity (PA), particularly free play and active transport.

Health promotion advocates an ecological multiple approach, based on different strategies and settings, first of all the school. It should be necessary not only expand Physical Education (PE) classes, but also: to provide active recess time; to make school facilities accessible before and after school time; to involve family and community. In this perspective, researchers recognize active Homework (HW) in PE as a good instrument to make children more active and aware of health advantages of an active lifestyle.

Within the activities of 'Movimentiamoci Project', carried out in 2012 in a number of primary schools of Abruzzo region (middle Italy), we created a PE Workbook (WB). That is an educational booklet containing different purposes of cognitive, affective and active HWs targeted to 6-8 years old children. It contains 20 modules, each composed of 3 units classified in 8 categories: Cognitive Learning, Self-Awareness, Creativity, Tradition, Swap it, Family, Affective Learning, Physical Exercise.

The objective of present study has been to evaluate the WB before using it, with the aim of improving it and forecasting the concrete impact 
on PE teachers' work and children's and families' attitudes and behaviours towards PA.

An 'a priori' evaluation based on experts' judgment, is being carried out. The Delphi procedure includes a series of questionnaires in subsequent rounds till the panel opinion will become definitively acceptable. At the moment 22 experts filled in the first questionnaire (Q1) answering 5-points Likert scales.

Statistical analysis compared median values and interquartile ranges with fixed cut-offs to establish the position and the agreement of judges' opinion on each issues.

Results from Q1 indicated that only 3 units have to be rejected, 32 units have to be changed with major revisions and 7 with minor revisions. The panel recognize 'Family' units very liking for children but less feasible in practice. The 'Tradition' category has been the most appreciated under all investigated aspects.

Overall, WB has been judged complete and very attractive for children in respect to iconographic character while the verbal language has to be improved.

Forecasting opinions were very positive about possibility that WB should increase actual children's PA, should improve children's enjoyment and attitude towards PA and should help teachers in performing PE program. They were less positive about parents' involvement and their behaviours change.

In conclusion, our study, with a very rigorous subjective opinion analysis, depicted WB as a good health promotion instrument and provided some important suggestions for improving it.

\section{Preventive use of physical activity education trial}

\section{A. Casagrande, M. Quarantelli, C. Galbusera}

\section{Facoltà di Scienze Motorie, Università San Raffaele Roma, Italia}

Back pain is one of the main causes of work disability. The regular practice of an adequate exercise program may reduce the incidence or, at least, the discomfort caused by back pain. The low level of motivation and the wariness towards any physical activity typical of subjects with rachialgia may prevent successful outcome of motor training programs and awareness of body movements. The aim of this study was to assess whether the practice of a specific and standardized training program in subjects with rachialgia leads to significant changes in pain, posture, spine flexibility and mobility, and activities of daily living (ADLs). Forty consecutive subjects with rachialgia were randomly assigned to a treatment group $(\mathrm{T})$ or to a control (C) group. Treatment consisted in "Pilates" training program. All subjects were evaluated by means of specific tests at the beginning and end of the trial. The subjects in the T group followed the assigned activity once a week for 10 weeks, and the evaluation tests were performed before and after each session. Sessions consisted in 45 min of Pilates activities. Subjects in the $\mathrm{C}$ group maintained their normal daily activities without any special training. Program and without the follow-up evaluations. Neck, back and low-back pain were evaluated by visual analog scale (VAS), range 0-10; functional disability by Quebec Back Pain Disability Scale (QBPDS). The tests performed were: bending test, spine rotation range, Unterberger test, anterior and posterior scapulum, hip asymmetry measurement. Statistical analysis was performed by paired t-test (within groups) and Anova (between groups). No difference was found between groups in gender distribution, age, height and body mass index. In the following lines some of the most representative results are shown as mean values \pm SEM

VAS for Neck pain changed from $3,80 \pm 0.97$ to $4,25 \pm 0,96$ (ns) in C, and from $6.18 \pm 0.81$ to $2.15 \pm 0.52(\mathrm{P}<0.001)$ in $\mathrm{T}$. VAS for back pain changed from $1,33 \pm 0.53$ to $1.65 \pm 0.50$ (ns) in $\mathrm{C}$, and from $2.63 \pm 0.73$ to
$0.85 \pm 0.36(\mathrm{P}<0.001)$ in $\mathrm{T}$. VAS for low-back-pain changed from $4.55 \pm 0.77$ to $5,33 \pm 0.84$ (n.s) in $\mathrm{C}$, and from $5.25 \pm 0.67$ to $2.08 \pm 0.39$ $(\mathrm{P}<0.01)$ in $\mathrm{T}$. Bending test changed from $-8.82 \pm 2.28$ to $-10.14 \pm 2.46(\mathrm{P}$ $<0.05)$ in $\mathrm{C}$, and from $-8.22 \pm 2.11$ to $-3.11 \pm 1.68(\mathrm{P}<0.01)$ in $\mathrm{T}$. QBPDS changed from $22.25 \pm 2$. to $27.50 \pm 2.65(\mathrm{P}<0.01)$ in $\mathrm{C}$, and from $19.95 \pm 3.14$ to $15.70 \pm 2.75(\mathrm{P}<0.01)$ in $\mathrm{T}$.

We conclude that a standardized training program may have positive effects on pain, functional disability and quality of life in back pain related to muscle imbalance, stiffness and posture defects. Standardized trials of physical activity education may play a role in a preventive dimension. Other training methods are being evaluated to be compared with the present data.

\section{Physical activity and self-esteem in childhood}

\section{Alesi ${ }^{I}$, A. Pepi ${ }^{I}$}

\section{${ }^{1}$ Dip. Psicologia, Università di Palermo, Italia}

Numerous developmental and educational studies assume that the selfesteem emerges from first school years when the child is required to compare his self-worth with peers and his actual performance on a variety of tasks such as physical or learning achievement. Anyway, this process of comparison between the perceived Self and the ideal Self develops during the life span and takes on crucial importance during pre-adolescence and adolescence. A variety of studies underlie the close and mutual relationship between self-esteem and body image from primary school on (Biddle and Asare, 2011; Sluijs, Kriemler and McMinn, 2012). According to Harter (1999), individuals make domain-specific judgements as well as global judgements of their selfworth and typically the evaluations they make vary across domains. Self-evaluations of physical appearance have been shown consistently to contribute strongly to global self-esteem. They represent important predictors of wellbeing as we age.

Body image is proved to be a significant developmental concern for adolescents and young adults, in light of physical, psychological and social transitions occurring during these periods and associations between body satisfaction and positive psychological and social functioning are empirically supported.

According to Harter's developmental model, this research aims to examine in more detail the relationships between the Self-esteem (Interpersonal, Body and School Self-Esteem) and the Self-Perception Profile in childhood as a function of gender.

The subjects in this study were 104 (59 males and 45 females) with an average chronological age of 11.26 years, practicing sport activities such as football, volley, swimming, basket.

Children were given the TMA (Bracken, 1992) to measure the self-esteem and the Self-Perception Profile Test (Harter, 1999). Were administered three subscales of TMA related to Interpersonal, Body and School Self-Esteem and all six subscales of Self-Perception Profile Test related to Scholastic competence, Athletic competence, Physical appearance, Peer acceptance, Behavioural conduct and Global competence.

On the whole, results show significant differences in Interpersonal, Body and School Self-Esteem as function of gender. Moreover were found positive correlations between body self-esteem and self-perception concerning athletic competence, physical appearance and global competence.

In conclusion, association between physical activity and psychological well-being in young people is evident, but studies in this domain are limited. Additional research is needed to examine in deep the role of educational agencies (family and school) to promote an "active sport model" from childhood. 
Time of day - effects on motor coordination and reactive strength in elite athletes and untrained adolescents

\author{
A. di Cagno ${ }^{1,2}$, C. Battaglia ${ }^{2}$, M.Piazza ${ }^{3}$, G. Fiorilli ${ }^{1}$, A. Giombini ${ }^{1}$, \\ G. Calcagno ${ }^{1}$, F. Pigozzi ${ }^{2}$, P. Borrione ${ }^{2}$
}

${ }^{1}$ Department of Medicine and Health Sciences, University of Molise, Campobasso, Italy

${ }^{2}$ Department of Health Sciences, Italian University of Sport and Movement of Rome, Italy

${ }^{3}$ Department of Anatomy, Histology and Forensic Medicine, University of Florence, Italy

The issue of time-of-day effects on performance is crucial when considering the goal of reaching peak results in sport disciplines. The present study was designed to examine time-of-day effects in adolescents on motor coordination assessed with Hirtz's battery and neuromuscular components of strength evaluated with reactive strength tests. Forty-two elite female gymnasts, aged $13.3 \pm 0.5$ years, were recruited for the study. Fifty healthy female students (aged $12.8 \pm 1.7$ years) served as the control group. All subjects underwent the testing sessions over two days at two different times of day in a randomized order. Half of the subjects were assessed early in the morning (8.30-9.30 a.m.) and subsequently in the late afternoon (7.00-8.00 p.m), while the remaining subjects were tested first in the late afternoon and then early in the morning. Oral temperature was measured at the two times of the day and a significant diurnal variation $(p<0.01)$ in both groups was found. MANOVA revealed significant group differences in the overall tests $(p<0.01)$. The gymnast group showed no significant differences in the coordination tests with respect to the time of day, but significant differences were observed for reactive strength as assessed with the vertical jump tests $(\mathrm{p}<0.01)$. Gymnasts attained better results in the afternoon in the reactive strength tests [flight time $(F 1.90=17.322 p<0.01)$ and ground contact time $(F 1.90=8.372 ; p<0.01)$ of the hopping test]. The temperature effect was more evident in the reactive strength tests and orientation test, especially in the gymnast group in which this effect added to their usual training time effect. The timesince-awakening influenced coordination performances in complex tasks more than reaction strength tests in simple tasks. The main outcome of the study was that we did not observe time-of-day effects on coordination skills in elite gymnasts and in untrained adolescents. The time of day in which athletes usually trained these skills could influence these results.

\section{References}

- Drust B, Waterhouse J, Atkinson G, Edwards B, Reilly T. (2005). Circadian Rhythms in Sports Performance - an Update. Chronobiol Int. 22:21-44.

- Souissi H, Chaouachi A, Chamari K, Dogui M, Amri M, Souissi N. (2010b). Time-of-day effects on short-term exercise performances in 10- to 11-year-old boys. Pediatr Exerc Sci. (4):613-623.

- Waterhouse J. (2010) Circadian rhythms and cognition. Prog Brain Res.185:131-53.

\section{Session 2. Functional Adaptation to Exercise}

\author{
Assessment of static and dinamic balance, spatio-temporal \\ and kinematics parameters, strength and power of lower limbs \\ muscle in a sample of elderly
}

F. D'Aliesio, D. Marandola, B. Federico, G. Capelli

Department of Human Sciences, Society, and Health University of Cassino and Southern Lazio, Via Sant'Angelo Località Folcara - 03043 - Cassino, Italy
Introduction and objectives: Aging is often associated with an increased risk of frailty related to gait disorders, loss of balance, gait tilting and a higher incidence of falls. The aims of study were the assessment of static and dinamic balance, spatio-temporal and kinematics parameters, strength and power of lower limbs in a sample of elderly.

Methods: This is a cross-sectional study, conducted on 45 elderly (12 men and 33 women), mean age 71.6 (range 59-82), attending a course of gymnastics in Cassino city (FR). The following parameters were analyzed: anthropometric features (weight and height), static and dinamic balance and walking ability (Tinetti test), physical and mental health (SF12 questionnaire), physical activity (PASE questionnaire - Physical Activity Scale for Elderly), strength and power of lower limb (Myorest Pro accelerometer), spatial and temporal parameters such as gait speed, stride length and step length (software Dartfish PRO 5.5).

Results: Gait speed was positively correlated with mental health and the power of the lower limbs muscle (Pearson's r 0.54 and 0.37 , respectively). Tinetti scale was weakly correlated with mental health and lower limbs muscle power $(r=0.24$ and $r=0.23$, respectively) and inversely correlated with age $(\mathrm{r}=-0.37)$. Physical activity was inversely correlated with BMI $(r=-0.46)$ and positively correlated with strength $(r=$ $0.34)$. BMI was inversely related to physical health $(r=-0.32)$ and positively to muscle strength $(\mathrm{r}=0.60)$.

Conclusions: The observed correlations were of medium or in low intensity. Longitudinal studies are needed to assess which parameters are associated with a lower fall risk in elderly.

\section{Effects of rowing training on airway responsiveness in asthmatics}

\section{Zangla ${ }^{1}$, N. Scichilone ${ }^{2}$, G. Morici ${ }^{3}$, R. Arrigo ${ }^{2}$, I. Cardillo $^{2}$, M. R. Bonsignore ${ }^{2}$}

\section{${ }^{1}$ Dip.to DISMOT, University of Palermo, Italy ${ }^{2}$ Dip.to DIMINIS, University of Palermo, Italy ${ }^{3}$ Dip.to BIONEC, Università di Palerm, Italy}

We previously reported that after a short course of rowing training the responsiveness to methacholine (Mch) in the absence of deep inspiration (DI) decreased in healthy subjects. We assessed whether a similar beneficial effect of exercise on airway responsiveness could occur in asthmatics. Nine patients (M/F: $3 / 6$; mean age \pm SD: $24 \pm 2$ yrs) with mild untreated asthma (FEV(1): $100 \pm 7.4 \%$ pred, $\mathrm{FEV}(1) / \mathrm{VC} 90 \pm 6.5 \%$ ) underwent a series of single dose Mch bronchoprovocations in the absence of DI in the course of a indoor rowing training consisted of two to three sessions per week for 10 weeks (total 28 sessions). Each session included a warm-up period for $20 \mathrm{~min}$ (running and stretching), and a specific training on rowing ergometer (Concept II, Morrisville, VT) for 40 to 70 minutes. In order to document power output, strokes, and mean maximal speed for each individual, a 1000-m all-out rowing test was first performed after a 20 min warm-up. Based on the performance, watt stroke1 and strokes min-1 recorded in the 1000-m all-out test, a certified European rowing coach created personalized training programs. At week 0,5 and 10,1000- and 2000-m all-out rowing tests were performed to evaluate performance and re-assess the individualized workload. The 10 -week training program included $20 \%$ of all training time at maximal power output, $10 \%$ of all training time at $75-90 \%$ of maximal power output and $70 \%$ of all training time at $60-65 \%$ of maximal power output. All sessions were supervised by a certified training instructor and a pulmonologist. The single dose Mch was established as the dose able to induce at least $15 \%$ reduction in IVC, and was administered to each subject at every challenge occasion. Five asthmatics (M/F: 1/4; mean age \pm SD: $26 \pm 3 \mathrm{yrs}$ ) with similar baseline lung function (FEV1: $102 \pm 7.0 \%$ pred, and $\mathrm{FEV}(1) / \mathrm{VC}: 83 \pm 6.0 \% ; \mathrm{p}=0.57$ and $\mathrm{p}=0.06$, respectively) not participating in the rowing training program, served as controls. In the trained 
group, the Mch-induced reduction in IVC from baseline was $22 \pm 10 \%$ at week $0,13 \pm 11 \%$ at week $5(\mathrm{p}=0.03), 11 \pm 8 \%$ at week $10(\mathrm{p}=0.028)$. The Mch-induced reduction in FEV(1) did not change with exercise $(p=0.69)$. The reduction in responsiveness induced by exercise was of the same magnitude of that previously obtained in healthy subjects $(50 \%$ with respect to pre-training). Conversely, Mch-induced reduction in IVC in controls remained unchanged after 10 weeks ( $\%$ reduction IVC at baseline: $21 \pm 20 \%$; after 10 weeks: $29 \pm 14 \%, p=0.28$ ). This study indicates that a short course of rowing training is capable of reducing airway responsiveness in mild asthmatics.

Effect of a long-term football training program on the expression of biomarkers related to oxidative metabolism in muscle biopsies

A. Alfieri ${ }^{1,2}$, P. Krustrup ${ }^{3}$, D. Martone ${ }^{1,2}$, A. Mancini $^{1,2}$, E. Imperlini ${ }^{2,4}$,

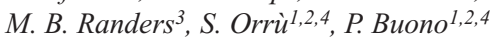

${ }^{1}$ Dept. of DiSIST, University "Parthenope", Naples, Italy

${ }^{2}$ CEINGE-Biotecnologie Avanzate s.c.arl., Naples, Italy

${ }^{3}$ Dept. of Exercise and Sport Sciences, University of Copenhagen, Copenhagen, Denmark

${ }^{4}$ SDN-IRCCS Foundation, Naples, Italy

Introduction: It is well known that the physical capacity is an important predictor of the risk of cardiovascular diseases (Pedersen and Saltin, 2006). Recent findings provide evidence that 2-3 weekly 1 hour football training sessions over 12 weeks have significant cardiovascular and muscular effects in untrained men; these positive adaptations are maintained over a 15 months period with a reduced training frequency (Krustrup et al. 2009 and 2010). We report here the effects of the long-term football program (8-a-side-football training program for 15 months), on the expression of biomarkers related to aerobic capacity and oxidative metabolism in muscle biopsies.

Methods: 5 young healthy untrained men were recruited by Prof. Krustrup's group. The participants carried-out a 64-week (15 months) football moderate-intense training program ( $80 \% \mathrm{Fc} \max ): 2.4$ sessions/week over the first 12 weeks, after which the training frequency was reduced to 1.3 times/week over the following 52 weeks. A muscle biopsy was obtained from the muscle vastus lateralis at $\mathrm{T} 0$ (before training) and after the intervention program (T1). Total RNA was extracted from muscle biopsies; quantification of gene expression was obtained by RTqPCR using the 2- $\Delta \Delta \mathrm{Ct}$ method as described in (Liss et al, 2002).

Results: The expression levels of PPAR $\gamma$, TFAM, NAMPT, PGC $1 \propto$, AMPK $\propto 1$ and AMPK $\propto 2$, SIRT1, Adiponectin and FABP4 messengers were determined on RNA from muscle biopsies of subjects at T0 and T1. Significant up-regulation of SIRT1 $(p<0.01)$ and PPAR $(p<0.0005)$ involved in the metabolism of glucose and fatty acids; TFAM $(\mathrm{p}<0.001)$, NAMPT $(p<0.01)$ and PGC1 $(p<0.01)$ directly or indirectly involved in the oxidative metabolism and in biogenesis and mitochondrial function; AMPK $\propto 1(p<0.01)$ and AMPK $\propto 2(p<0.0005)$, that enhanced oxidative metabolism and mitochondrial biogenesis in trained subjects (T1) to respect to untrained (T0) was found. No significant increase in Adiponectin and FABP4 expression levels at T1 to respect to T0 was found.

Quantitative expression level of selected biomarkers in muscle biopsies from untrained (T0) and 15-months trained subjects (T1).

Data represent the mean (+/-SD) of 3 different experiments. Data are analyzed with the Student's t-test. Differences were considered significant at $\mathrm{p}$-value $<0.05$.

$\mathrm{p}<0.01, \mathrm{p}<0.001$ and $\mathrm{p}<0.0005$ represent statistical differences of the expression level of each messenger at T1 vs T0. Arbitrary value 1 was assigned for the level expression of each messenger at $\mathrm{T} 0$.

In conclusion, a moderate-intense long-term football training program is able to provide health benefits by inducing in the skeletal muscle the upregulation of specific biomarkers directly or indirectly involved in the aerobic oxidative capacity and in the oxidative metabolism of glucose and fatty acids.

Combined effects of temperature manipulation and fatigue on skeletal muscle electrical and mechanical characteristics

\author{
E. Cè, E. Limonta, S. Rampichini, L. Agnello, F. Esposito
}

Department of Biomedical Sciences for Health, University of Milan, Milan, Italy

Muscle cooling and fatigue are two factors which induce similar effects on muscle sarcolemmal propagation properties and force output, thus affecting the electrochemical and mechanical characteristics of a contracting muscle. The aim of the study was to assess the combined effect of muscle temperature $(\mathrm{Tm})$ manipulation and fatigue on skeletal muscle electromechanical characteristics during isometric contraction. After maximum voluntary contraction (MVC) assessment, 16 participants performed brief isometric tasks of different intensities in low (TmL), high $(\mathrm{TmH})$ and neutral $(\mathrm{TmN})$ temperature condition, before and after a fatiguing exercise. During contraction, the surface electromyogram (EMG), mechanomyogram (MMG) and force (F) signals were recorded from the biceps brachii muscle. The root mean square (RMS) and conduction velocity (CV) of EMG signal, the peak-to-peak (p-p) of MMG signal, the rate of force development (RFD) and the half-relaxation time (HRT) of F signal were calculated. The electromechanical delay (EMD), the latencies between EMG and MMG ( $\Delta$ t EMG-MMG, electrochemical component of EMD), and MMG and F ( $\Delta \mathrm{t}$ MMG-F, mechanical component of EMD) were also identified. After the fatiguing exercise: (i) MVC,RFD, HRT, EMG RMS, MMG p-p, EMD and its components were affected to a similar extent by fatigue $(\mathrm{P}<0.05)$; (ii) $\Delta \mathrm{t}$ EMG-MMG lengthened significantly under $\mathrm{TmL}$ compared to $\mathrm{TmN}$ and $\mathrm{TmH}(\mathrm{P}<0.05)$; (iii) $\mathrm{CV}$ decreased to a further extent in TmL compared to $\mathrm{TmN}$ and $\mathrm{TmH}(\mathrm{P}<0.05)$. The fatigue-induced $\Delta \mathrm{t}$ EMG-MG lengthening and the larger $\mathrm{CV}$ drop in TmL suggest that cooling and fatigue have a combined and additional effect on sarcolemmal propagation properties but not on muscle mechanical behavior and maximum force generating capacity.

\section{Analysis of skeletal age's differences and the relative age effect between two level of selection (national and regional) of young athletes}

\section{A. Brighenti ${ }^{1}$, A. Savoldelli ${ }^{1}$, R. Modena ${ }^{1}$, B. Pellegrini ${ }^{1}$, F. Schena ${ }^{1}$ \\ ${ }^{1}$ CeRiSM (Research Centre of Sport Mountain and Health) University of Verona, Italy}

Introduction: It could be hypothesized that the biological athlete's development influences the selection level of national and regional high performance selected athletes. The aim of this study is to compare the skeletal age (SA) and chronological age (CA) of several athletes selected in national (ITA) and regional (TN) teams, and then to observe the quarterly distribution of the birth date to examine the hypothetic effect of relative age on results[1].

Methods: 96 athletes of the Italian teams ( 67 female and 29 male age $15.54 \pm 1.22 \mathrm{y}$ ) and 46 athletes of the regional teams ( 35 female and 11 male age $14.35 \pm 1.38 \mathrm{y}$ ) both belonging to different sports federations participated to the study. The SA data were collected with Bone Profiler® (I.G.E.A. Italy) in CeRiSM (University of Verona) and the data analysis was made through student T.Test. 
Results:: SA was found to be $0.79 \pm 0.98$ y and $1.11 \pm 0.88$ y higher than CA respectively for TN and ITA and the significance of the difference between this two groups was $\mathrm{p}=0.0504$.

The results of the analysis of the quarterly distribution of birth dates are expressed in percent of the total: $1^{\circ}$ quarter $(29 \% \mathrm{ITA}-35 \% \mathrm{TN}) ; 2^{\circ}$ quarter $(20 \% \mathrm{ITA}-24 \% \mathrm{TN}) ; 3^{\circ}$ quarter $(24 \% \mathrm{ITA}-27 \% \mathrm{TN}) ; 4^{\circ}$ quarter (27\%ITA - 14\%TN).

Conclusion: the results suggest that the athletes selected in the national teams presented an earlier biological maturation with respect to the regional teams, however the significance was not reached and this leads to refute the initial hypothesis.

The relative age effect was detected only in regional teams. We could hypothesized that the greater number of people who practice sports in the whole nation compared to in a reduced area, permits to find athletes biologically developed born at any time of the year.

The next step would be to measure a group of not sport selected people of the same CA to deepen the matter.

References

1. Mujika, I., et al., The relative age effect in a professional football club setting. Journal of sports sciences, 2009. 27(11): p. 1153-1158

\section{Effect of 4 and 8 weeks of play-based physical activity} on metabolomic signatures in overweight adolescents

\author{
M. Meucci ${ }^{1}$, C. D Curry ${ }^{2}$, C. Cook ${ }^{2}$, C. Baldari ${ }^{1}$, L. Guidetti ${ }^{1}$, W. Jia ${ }^{3}$, \\ S. Collier $^{2}$ \\ IDept of Health Science, University of Rome "Foro Italico", Rome, \\ Italy \\ ${ }^{2}$ Dept of Health, Leisure, and Exercise Science, Appalachian State \\ University, Boone, NC \\ ${ }^{3}$ Dept of Nutrition, University of North Carolina at Greensboro, \\ Greensboro, NC
}

Background: Recently, The American College of Sports Medicine related the obesity epidemic in children to a sedentary behavior, which may be more prevalent during the summer break from school. Play-based activities may be the best strategy to increase activity throughout summer, yet the metabolic signatures and the dose response of activity has never been elucidated. Urinary metabolic signatures are a non-invasive method to study the shifts in metabolic which may identify the mechanisms underlying the physiological changes from play-based activity.

Purpose: The aim of this study was to elucidate the effect of 4 and 8 weeks of play-based activity on urinary metabolomic signatures in overweight adolescents.

Methods: Twelve recreationally active adolescents were recruited in two weeks from the local community via flyers and only healthy, recreational active adolescents from 8 to 12 years old were chosen. Participants were randomly divided into a 4 or 8 week supervised activity group $(4 \mathrm{w}$, $\mathrm{n}=6$ or $8 \mathrm{w}, \mathrm{n}=6$ respectively) with exercise activities aimed to teach new skills and increase strength, flexibility and cardiovascular fitness through play-based activity. Subjects reported twice to the Vascular Biology and Autonomic Studies Laboratory, before and after 4 or 8 weeks depending on their group assignment. During each visit, urinary samples were collected followed by anthropometrics and a peak oxygen consumption (VO2peak) measurement. Pre- and post-exercise samples in both $4 \mathrm{w}$ and $8 \mathrm{w}$ groups were analyzed through a partial least squares-discriminant analysis (PLS-DA) models and a 2x2 (group by time) ANOVA with repeated measures was employed on VO2peak data.

Results: A distinct metabolic shift between pre- and post-exercise conditions was observed in the PLS-DA model after 8 weeks of exercise $(\mathrm{R} 2 \mathrm{X}=0.388, \mathrm{R} 2 \mathrm{Y}=0.862, \mathrm{Q} 2=0.144)$ supported by a significant increase in VO2peak $(\mathrm{P}=0.001)$. No valid PLS-DA models or significant changes in VO2peak were obtained between pre- and post-exercise subjects of the 4-week group.

Conclusion: 4 weeks of play-based activity may not be sufficient to affect the metabolic signatures that we assayed, however 8 weeks of activity significantly affected metabolic pathways resulting a distinct shift in the metabolome with a concomitant improvement in aerobic fitness.

\section{Neuromuscular electrical stimulation is able to counteract Sarcopenia? A case report}

\section{S. Fulle, T. Pietrangelo, R. Mancinelli, G. Fanò-Illic}

Dipartimento di Neuroscienze \& Imaging, Università " $G$. d'Annunzio", Chieti-Pescara, Italy

Unità Operativa di Fisiologia Clinica, CRC-CeSI, Fondazione

"G. d'Annunzio", Chieti-Pescara, Italy

Istituto Interuniversitario di Miologia, Università “G. d'Annunzio", Chieti-Pescara, Italy

The neuromuscular electrical stimulation (NMES) has been acknowledged as an efficient modality leading to significant improvements in isometric maximal voluntary strength, while the effects of NMES on muscle fatigability are still poorly understood and required further investigations. NMES is also employed in rehabilitation protocols to treat muscle atrophy deriving by different physiological (sarcopenia) or pathological (disuse, cachexia., ecc..) reasons in which, however, the regenerative skeletal muscle system is involved. Aims of this paper is to analyze the behavior of the regenerative system of the VL muscle (satellite cell pool) after NMES training in a 64 years old, healthy (but sarcopenic), volunteer. Training consisting in 24 sessions during 8 weeks to $18 \mathrm{~min}$ per session (40 contractions 6.25 seconds each) Parameters were: frequency $75 \mathrm{hz}$; pulse duration $400 \mathrm{msec}$; intensity variable set at $60 \%$ maximal isometric voluntary contraction. Needle biopsies $(4-10 \mathrm{mg})$ were taken on VL muscle before and after the training. Results: The NMES actived recruitment of satellite cells that showed a very high proliferative rate with a pdl index higher than the control. The recruitment and the activation of satellite cells is also demonstrated by the presence of a greater number of myocytes (and probably fibers) pax-7 positive. In additions, very interesting data derived from antioxidant scavenger system analysis which not only was activated by NMES but was also able to offer synergies with the presence of natural antioxidants such as cranberry extract. Molecular analysis of transcriptome seems to confirm this scenario.

\section{Neuromuscular activation profile of vastus lateralis in soccer players during a competitive match}

\section{Montini, A. Rossetta, F. Felici, M. Sacchetti, I. Bazzucchi}

Department of Human Movement and Sport Sciences, University of Rome "Foro Italico", Italia

Introduction: The physical aspects of soccer have been studied intensively using time-motion analysis. The current match-analysis techniques allow detailed tracking of player movement but the onset and development of muscle fatigue during the match are difficult to establish.

Aim: The present study aimed to investigate the neuromuscular activation profile throughout a competitive soccer match.

Methods: Ten amateur soccer players (age 18.1 \pm 0.7 years; height $1.78 \pm 0.07 \mathrm{~m}$; mass $69.0 \pm 9.5 \mathrm{~kg}$ ) were recruited for this study. Electromyographic (EMG) activity of the vastus lateralis muscle was 
recorded during four unofficial matches. Signals were collected simultaneously from the players by means of 10 EMG micro-amplifiers. Moreover, players were requested to perform a first maximal voluntary isometric contraction before the match (MVCpre) and a second immediately after the end of the match (MVCpost). The root mean square (RMS) of the EMG signal recorded during the match was analysed offline and normalized for the maximal RMS obtained during the MVCpre (100\%RMSmax). Six categories were created in order to represent the \%RMS distribution during the match (1st: 0-20\% RMSmax; 2nd: $20-40 \%$ RMSmax; 3rd: 40-60\% RMSmax; 4th 60-80\% RMSmax; 5th $80-100 \%$ RMSmax; 6th: 100-120\% RMSmax).

Results: Knee extensors torque showed a non significant decrease ($5 \%$ on average) after the match. Conversely, RMS recorded during the MVCpost was significantly lower $(p<0.01)$ than RMS at MVCpre $(-$ $14 \%$ on average). The mean total distribution of $\%$ RMS of the players during the matches was: $85.3 \pm 1.7 \%$ in the 1 st category, $8.3 \pm 1.5 \%$ in the $2 \mathrm{nd}, 3.5 \pm 0.2 \%$ in the $3 \mathrm{rd}, 1.7 \pm 0.2 \%$ in the 4 th, $0.8 \pm 0.2 \%$ in the 5 th and $0.4 \pm 0.1 \%$ in the 6 th.

Conclusion: This is the first study which assessed EMG signals throughout a soccer match-play. Neuromuscular activation profile of the match will help to organize training protocol, since there is a consensus in sport science that the most effective training for preparing athletes for competition is that which most closely replicates competitive performance conditions.

\section{ORAL PRESENTATIONS}

\section{Saturday, October $6^{\text {th }}, 2012$}

\section{Session 3. Training \& Performance}

\section{Decision making in handball players: situational probabilities and contextual information in complex problem solving}

S. Nicolosi, S. Cilia, R. Schembri, S. Di Stefano, M. Lipoma

Facoltà di Ingegneria, Architettura e Scienze motorie, Università di Enna Kore, Italia

Introduction: Research on decision making in sport investigated the relationships between perception, cognition and performance, the study of pattern recall or recognition, and choice-reaction paradigms. Few studies attempted to analyze the knowledge of situational probabilities and its relationship with decision-making processes in sport (Williams and Ward, 2007).

Objective: The purpose of this study was to analyze the reasoning ability about probabilities in the processing of situational and contextual informations and to analyze the strategies used by handball players in solving problems sport-related. It aimed to verify how experts, compared to less experienced, judiciously determine the importance of each potential option presented, effectively seeking new information and extracting them from observation.

Method: 45 subjects, including 22 females and 23 males, who play handball in series A1 and A2 were involved. Video on 4 action game, played by top professionals, and observation schedule sheets were used to record and assess the responses of the players.

Videos selected, and evaluated by the team coaches, were concerned four situational game problems, such as attack/defense related to the numerical control of the opponents (inferiority/equality/superiority numerical conditions) and were administered with alternate combinations.

Participants were asked to examine sequences associated with the game, predicting the possible actions related to a player, who was indicated in the video with a red arrow.

Other instruments were used to assess cognitive skills in attention, working memory, and reasoning, including: Block Design and Arithmetic subtests of WAIS-R by Wechsler (1981), Attention and Concentration Test: Choice-reaction Times, Digit Span, and Shifting Attention Tasks by Di Nuovo (2000).

Results: Skilled players were more accurate in description of actions and technically more proficient in predicting actions, when problem was more complex (situation 4). Results provide a qualitative description of situational probabilities and decision making processes sport-related. Findings confirm previous research that has been demonstrated expert superiority in different aspects of decision making process (BarEli et al., 2011; Mann et al, 2007; Baker et al. 2003; Ripoll et al.,1995). However, little is yet known about how such abilities develop and how we can effectively train them. In fact there are few longitudinal studies that observe these aspects. Further studies could assess the effectiveness of possible training methods of anticipation, reasoning and decision of athletes with different levels of expertise. 


\section{Evaluation of a shuttle running test in young soccer players}

G. Condello, F. Di Giuseppe, R. Messina, A. Tessitore

Department of Human Movement and Sport Sciences, Università degli Studi di Roma “Foro Italico”, Rome, Italy

Introduction: Rapid accelerations and decelerations, as well as directional changes, are fundamental abilities in soccer players1. Moreover, their improvement is necessary for young athletes to reach a high level of movement mastery. Thus the purpose of this study was to assess a shuttle running test and its relationship with a straight sprint.

Methods: Thirty soccer players (age $=12$ years) performed a $15 \mathrm{~m}$ straight sprint (15SS) and a $15 \mathrm{~m}$ shuttle running test (15SRT) comprised of two splits of $7.5 \mathrm{~m}$ distance with a $180^{\circ}$ change of direction. Total time for each test as well as first split (S1), inversion (INV) and second split (S2) time for 15SRT, were recorded by means of a dual infrared photoelectric cells system. Relationship between the tests was calculated using Pearson's correlation coefficient. One way ANOVA was applied to verify the difference between the two splits of 15SRT.

Results: A significant correlation was found between 15SS and 15SRT $(r=0.66)$. No difference emerged between splits.

Discussion: The two tests shared only $44 \%$ of common variance suggesting that the two movements cannot be considered as similar abilities. There was a lack of a significant difference between split times, even if they involved different tasks. In fact, S1 required coupling of an acceleration and deceleration phase while $\mathrm{S} 2$ consisted of a progressive acceleration. It might be speculated that the athletes had poor abilities to rapidly accelerate and decelerate based on growth and maturation aspects. In fact, previous studies suggested an approximate age period from 13.5 to 14.5 years during which an increase in physical performances (i.e., speed, agility, explosive power, aerobic endurance, anaerobic capacity) is reasonable2,3. Similar finding emerged for INV. In fact, the range values $(0.35-0.88 \mathrm{sec})$ revealed how some players already had a good ability to quickly change direction while others still had to develop it. However it can be considered acceptable that a high level of movement mastery has not yet been achieved at this stage of age. Thus, a multi-year youth training plan has to provide the correct exercise progression to increase the ability to perform efficient accelerations, decelerations, and changes of direction.

References

1. Tessitore A, Picerno P, Meeusen R, Zok M, Capranica L. Relationship between sprinting in straight line, changing direction, and alternating quick accelerations and decelerations in soccer players. Book of Abstracts of "VIII World Congress of Performance Analysis of Sport", 2008

2. Philippaerts RM, Vaeyens R, Janssens M, Van Renterghem B , Matthys D, Craen R, Bourgois J, Vrijens J, Beunen G, Malina RM. The relationship between peak height velocity and physical performance in youth soccer players. J Sports Sci. 2006;24:221-230

3. Gil S, Ruiz F, Irazusta A, Gil J, Irazusta J. Selection of young soccer players in terms of anthropometric and physiological factors. J Sports Med Phys Fitness. 2007;47:25-32

\section{Differences in adaptations to slope in classical cross-country ski- ing techniques}

\author{
B. Pellegrini ${ }^{1,2}$, C. Zoppirolli ${ }^{1,2}$, L. Bortolan ${ }^{1,2}$, P. Zamparo ${ }^{2}$, F. Schena ${ }^{1,2}$ \\ ${ }^{1}$ CeRiSM ( Research Center of Sport Mountain and Health), \\ University of Verona, Rovereto, Italy \\ ${ }^{2}$ Department of Neurological, Neuropysichological, Morphological \\ and Movement Sciences, University of Verona, Italy
}

Introduction: In classical cross country skiing the main techniques are diagonal stride (DS), that is performed by pushing alternatively with skis and poles and double poling (DP) in which the propulsion is demanded by a synchronous action of both poles. DP is adopted on flat and for fast skiing, and its importance has increased in recent years, however DS is preferred on uphill tracts. The aim of this study was to describe the cost of locomotion as well as the timing and the amount of propulsion for the two techniques and to analyse the adaptations that occur when the slope increases.

Methods: Ten cross-country skiers were asked to perform DP and DS with roller-skis on a treadmill at $10 \mathrm{~km} / \mathrm{h}$ at 5 slopes from $0^{\circ}$ up to $4^{\circ}$. The cost of locomotion (C) was calculated by measuring oxygen uptake at steady state and blood lactate at the end of each bout. The motion of skis and poles were acquired using a motion capture system and the force exerted through the poles was measured by means of load cells inserted into the poles. The cycle time (CT), the absolute and relative duration of poling phase (PT, $\mathrm{PT} \%$ ), the duration of the leg thrust phase (TT, TT\%, for DS only), the force exerted trough the poles (PF) were computed. A 2 way (slope $\mathrm{x}$ technique) ANOVA was applied; level of significance was set at 0.05 .

Results: The C was lower for DP than for DS on flat $(2.29 \pm 0.33 \mathrm{Jkg}-$ $1 \mathrm{~m}-1$ vs $3.03 \pm 0.46 \mathrm{Jkg}-1 \mathrm{~m}-1)$; it increased linearly with slope with an higher rate for DP $\left(1.0 \mathrm{Jkg}-1 \mathrm{~m}-1{ }^{\circ}, \mathrm{R} 2=0.99\right)$ than for DS $(0.674 \mathrm{Jkg}-$ $\left.1 \mathrm{~m}-1 /{ }^{\circ}, \mathrm{R} 2=0.99\right)$. CT was about $1.2-2 \mathrm{~s}$ and decreased linearly with slope with higher rate for DP $\left(0.20 \mathrm{~s} /{ }^{\circ}, \mathrm{R} 2=0.97\right)$ than for DS $\left(0.04 \mathrm{~s} /{ }^{\circ}\right.$, $\mathrm{R} 2=0.92)$. PT values doesn't changed with the slope and were longer for DP than for DS ( $\sim 0.58 \mathrm{~s}$ and $\sim 0.46 \mathrm{~s}$ respectively). PT\% increased linearly with the slope, with higher rate for DP $\left(3.5 \% /^{\circ}, \mathrm{R} 2=0.97\right)$ than for DS $\left(1.14 \% /{ }^{\circ} \mathrm{R} 2=0.90\right)$. TT and TT\% increased linearly (R2 $=0.97$ and $\mathrm{R} 2=0.98$ ). The value of $\mathrm{PF}$ at each slope and rate of increase were higher for DP than DS. The percentage values at $4^{\circ}$ with respect to $0^{\circ}$ was $269 \%$ and $186 \%$ for C, $320 \%$ and $150 \%$ for PF, $61 \%$ and $88 \%$ for CT, $148 \%$ and $110 \%$ for PT $\%$ for DS and DP respectively, $152 \%$ for TT and $170 \%$ for TT $\%$ for DS.

Conclusion: The increase of workload induced by the increase of slope is faced by changes in the cost of locomotion, in the timing and force of propulsion that are different between the techniques. DP is the less expensive technique on flat on flat but the energy cost of this technique rises more rapidly than for DS. This is associated with a greater adaptation of the parameters related to the propulsive action trough the pole for DP with respect to DS. In particular, the greater increase of PF in DP could be the limiting factor in the use of this techniques for uphill skiing. In DS, the contribution of leg trust phase, which duration increased with slope, led to a lower increase of $\mathrm{C}$ and to a better sustainability of this technique at increasing slopes.

\section{Can the knowledge of chronotype be useful for the motivation and the training plans?}

\section{J. Vitale ${ }^{1}$, D. Formenti ${ }^{2}, A$. Weydahl $^{3}$, G. Alberti ${ }^{2}$, F. $_{\text {Carandente }}^{2}$}

${ }^{1}$ Faculty of Exercise and Sports Science, Università degli Studi di Milano, Italia

${ }^{2}$ Department of Biomedical Sciences for Health, Università degli Studi di Milano, Italia

${ }^{3}$ Finnmark University College, Alta, Norway

Introduction: General knowledge of "time of day" effects on exercise might not be enough, since everyone can have a different circadian propensity (chronotype) based on their position on a MorningnessEveningness Scale (ranges from a morning-type = M-type to eveningtype $=\mathrm{E}$-type, or neither-type $=\mathrm{N}$-type) (3). In this project we studied the response to the same physical activity performed in the morning 
and evening by different chronotypes.

Methods: Each of 22 subjects (12 males and 10 females, age: $23.2 \pm$ 3.6, BMI: $22.45 \pm 2.7$ ) filled out the Horne-Ostberg MorningnessEveningness Questionnaire (MEQ) and the results have been compared to the Morningness-Eveningness Scale to determine their chronotype (3). They then performed, at their own voluntary speed, a walking session, consisting of three repetitions uphill and downhill, first in the late afternoon at 16:30h and then the next morning at 8:30h. The performance time was recorded by an EKT-orienteering system. Each subject wore a HR monitor (PolarTeam 2) to record their HR during each entire walking session and reported their perceived exertion (Borg scale 6-20) upon completion (1).

Results: $\mathrm{MEQ}=14 \mathrm{~N}$-types, 4 E-types and 4 M-types. Results from one M-type, one E-type and two N-types (as controls) are discussed in detail for the case-study. The M-type walked faster in the morning than in the late afternoon, but showed no difference in HR response. Conversely, the E-type walked faster in the evening than in the morning, with similar HR in both sessions. The N-types showed increased HR when performance time decreased.

Conclusion: This case-study showed that response to physical activity can be influenced by chronotype, in agreement with other reports in the literature (2),(4). While a follow-up study with more subjects is necessary, the results suggest that when trying to increase the health and well-being in the population, it would be advisable to arrange physical activity classes both in the morning and in the evening, and have the public join the classes according to their chronotype to achieve the most benefit.

References

1. Borg, G. A. (1982). Psychophysical bases of percieved exertion. Medicine and Science In Sports and Exercise, 14(5), 377-381

2. Brown, F. M., Neft, E. E., \& LaJambe, C. M. (2008). Collegiate rowing crew performance varies by morningness-eveningness. J Strength Cond Res, 22(6), 1894-1900

3. Horne, J. A., \& Ostberg, O. (1976). A self-assessment questionnaire to determine morningness-eveningness in human circadian rhythms. Int J Chronobiol, 4(2), 97-110

4. Sugawara, J., Hamada, Y., Nishijima, T., \& Matsuda, M. (2001). Diurnal variations of post-exercise parasympathetic nervous reactivation in different chronotypes. Jpn Heart J, 42(2), 163-171

\section{Performance characteristics of a high-level fencer, competing proposals for the training, analysis of workout activities}

\section{Omeri ${ }^{1}$, C. Galbusera ${ }^{2}$, M. Quarantelli ${ }^{2}$ \\ ${ }^{1}$ Società Sportiva Scherma Brescia, Italia \\ ${ }^{2}$ Università San Raffaele Roma, Italia}

The present study proposes a recognition of the literature regarding the definition of a performance model of fencing.

In particular, it analyzes the latest researches that promote reflection and reconsideration of the performance model of the High-level fencer that was always identified as a "sport of skill with a strong muscular component."

It is presented a study that defines a new protocol and valid evaluation tests specifically for fencing, which has the aim to investigate the endurance of the fencer.

The findings help us to focus on "integrated model of fencing performance", which seems to express organic, neuromuscular, sports-specific components. Also allows to consider that stimuli motor, cognitive activity alone fencing does not appear sufficient to ensure adequate support to the needs of training and competition for High Level athletes. The second part presents a testing protocol designed with a High-Level
Athlete whose results appear to confirm definitely the most current information.

The reflections from the deepening induced by the literature and experimentation should prompt professionals (coaches, trainers) and the athletes to reconsider content, strategies, planning of training in relation to an effective integration of specific stressors, special and general.

\section{Repeated bout effect induced by eccentric isoinertial device}

G. Coratella ${ }^{1}$, A. Chemello ${ }^{1}$, F. Schena ${ }^{1,2}$, F. Impellizzeri ${ }^{2}$

${ }^{1}$ Dipartimento Scienze Neurologiche, Neuropsicologiche, Morfologiche e Motorie, Università di Verona, Italia

${ }^{2}$ Centro Ricerche Sport, Mountain and Health, Rovereto, Italia

Muscle damage is proved to occur after eccentric training (Clarkson, 2002). Moreover, it confers protection to a subsequent session, i.e.: repeated bout effect (Nosaka 2001). A new technology (YoYo technology) has been developed in order to enhance negative phase using inertia (Tesch 1994). Aim of the study is evaluate if lengthening contraction enhanced by inertial flywheel squat will cause symptoms of muscle injury and following protection.

Twelve healthy males performed 100 maximal squat repetitions using inertial device. At baseline, after training and up to 4 days after CK blood concentration, Knee extensors strength, muscle soreness and jump performance were measured as markers of muscle damage. Same protocol was repeated after 3 weeks. Statistical analysis was performed using repeated measures ANOVA, using factor Session and Day.

No significant Session X Day interaction occurred in all parameters. Single factor Session and Day were significant except in [CK]. Post hoc analysis revealed, compare to baseline and after first trainng, [CK] and soreness increments respectively up to 3 and 4 days, while strength loss and jump height were affected only post training. The second bout revealed significant markers decrements compared to the first one in CK (-50\% and $-43 \%$ after 2 and 3 days), muscle soreness $(-2,8 ;-2,6$; 3,$4 ;-2,6$ A.U. respectively after $1,2,3$ and 4 days) and strength ( $12 \%$; $9 \% ; 15 \% ; 15 \% ; 14 \%$ respectively immediately after training, after 1,2 , 3 and 4 days).

Inertial flywheel squat induce classical symptoms of muscle injury, even if strength loss and performance could be positively influenced by neural patterns. CK blood activity is relatively lower compared to single joint exercise because of load is divided among more muscles. However, eccentric overload does protect muscle at least up to 3 weeks. Trainers and conditioners should take in account the recovery time course after eccentric inertial squat.

References

- Clarkson PM et al. (2002) Exercise Induced Muscle Damage in Humans. American journal of physical medicine \& rehabilitation, 81(11)S: S52-69

- Nosaka K et al.(2001) How long does the protective effect on eccentric exercise induced muscle damage last?. Medicine \& Science in Sports \& Exercise, 33(9): 1490-95.

- Tesch P et al.(1994) A gravity-independent ergometer to be used for resistance training in space. Aviation, space, and environmental medicine, 65(8): 752-6

\section{The incidence of ball handling on swimming times in women waterpolo}

D. Tursi, S. Napolitano, P.A. Di Tore, G. Raiola

Facoltà di Sceinze della Formazione, Università di Salerno, Italia 
The purpose of this study was to assess the impact of the ball handling on swimming times in women waterpolo, in order to obtain useful information to define training methods. Eleven high-level athletes were enrolled, which was required to perform the test speed of 300 ( 15 repetitions of 20 meters), once with the ball, and a second time without the ball.

For each athlete has been calculated the mean and standard error for test, with and without the ball. The analysis was carried out individually for each athlete, and in total for each test. The comparison of the results indicates a high variability, and indicates a non-mechanical incidence of ball-handling swimming times.

Analysis of the data collected shows that the water polo players of the highest level (Starace and Valkai) do not have significant changes in chronometric, while, for the athletes coming from competitive swimming (Guillet and Giuliani), the ball-handling has a clear and negative impact on swim development.

For the athletes coming from water polo (Anastasio, Pilgrim), ball-handling affects swimming times in a positive way (results indicate fastest times in the tests carried out with the ball), while for the other athletes the results do not show significative changes.

The results show that this study will help the coach to develop a training methodology effective in improving performance. Coaches is suggested to increase the use of ball-handling in all conditions of training.

\section{The effect of the step frequency manipulation on the step length in marathon runners}

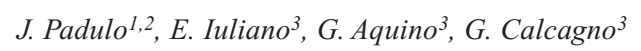

${ }^{I}$ Faculty of Medicine and Surgery, University of "Tor Vergata" Rome, Italy

${ }^{2}$ CONI - Italian Regional Olympic Committee, Sardinia, Cagliari, Italy

${ }^{3}$ Department of Medicine and Health Sciences, University of Molise, Italy

The present study investigated the effects of step frequency manipulation during training in slopes $(2 \%)$ on footstep at Iso-Efficiency Speed (IES) (Padulo et al., 2012). Twenty-four male marathon were randomly allocated to one of two training groups for three weeks: step frequency manipulation group (SFM, $\mathrm{n}=12$ ) and free step frequency group (SFF, $\mathrm{n}=12$ ). The SFM performed with 5 sets of 5 minutes of running $2 \%$ slope at IES with a manipulated step frequency (Padulo et al., 2011) (calculated in order to replicate the same step length elicited during level) while in SFF the step frequency was freely chosen. A digital cameras $(210 \mathrm{~Hz})$ was used to record and software (Dartfish 5.5Pro) was used to perform a $2 \mathrm{D}$ video analysis. ANOVA with repeated measures and Bonferroni post hoc showed significant differences between both training groups in step length: $F(1,22)=28.09, p<0.001(\eta 2=0.569)$, and the interaction training type $\mathrm{x}$ time $\mathrm{F}(1,22)=58.50, \mathrm{p}<0.001(\eta 2=$ $0.727)$. In SFM group showed a increased in step length $4.30 \%$ ( $<<$ 0.001 ) while in SFF was $<1 \%$ (n.s.) after the training vs baseline conditions. These findings coincide with characteristics of better running performances. The results from the study could help coaches to devise training methods which could improve an athlete's performance through increasing step length. The method provided may aid faster race times for athlete's.

\section{References}

- Padulo, J., Annino, G., Migliaccio, G. M., D’Ottavio, S. \& Tihanyi, J. (2011). Kinematics of Running at Different Slopes and Speeds. J Strength Cond.Res., 26, 1331-1339

- Padulo, J., Annino, G., Smith, L., Migliaccio, G. M., Camino, R., Tihanyi, J. \& D’Ottavio, S. (2012). Uphill Running at Iso-Efficiency Speed. Int J Sports Med
The Performance analysis in volleyball: study on the usefulness of the libero

\section{P. Mango, F. Sgrò, M. Barresi, S. Pignato, M. Lipoma}

Engineering, Architecture and Physical Activities Faculty, University of Enna, Enna, Italy

Introduction: In modern volleyball the player called "libero" represents one of the targets of the international federation to give a shape to an overspecialized player in defensive skills. Libero's performances, during a match, should improve the team's performance in catching and defensive actions.

Aims: The following research questions were defined considering the restrictions to libero's actions:

-)What is the relevance of an overspecialized player in volleyball?

-)Does libero represent a strong point for team's performance?

Methods: Results presented were obtained applying a sport performance analysis methodology on five matches of an italian male team attended to the latest A2 Serie championship. We used a computerized match analysis methodology in order to compare the libero's performances with the ones of his teammates, analyzing catching and defensive fundamentals. The ground was divided into eighteen cells and the qualitative analysis of each action was codified using categorical labels. For our purpose we used the following software: Kinovea, Scout Line, and Microsoft Excel.

Results and discussion: Findings showed that libero's performance don't strengthen team outcome, on the contrary the restriction of his actions wouldn't justify the presence of a player specialized in defensive skills .

In order to generalize our results we are currently expanding the sample, hypothesizing a comparison according to different level and gender.

\section{Central and peripheral contribution in neuromuscular fatigue during ultra-endurance road cycling: a case study}

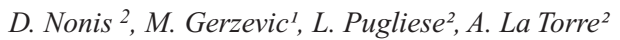

${ }^{1}$ Institute of Kinesiology Research, Science and Research Centre of Koper, University of Primorska

${ }^{2}$ Department of Biomedical Sciences for Health, University of Milan, Italy

Introduction: Ultra-endurance (UE) cycling is a very demanding discipline poorly studied, considering a few data published in the literature with increased numbers of amateurs involved. The fatigue is usually considered as a reduction of maximal force that a muscle can exert, divided to central and peripheral fatigue in function where the impairments are allocated (1). The aim of this case study was to investigate the neuromuscular fatigue induced by an UE cycling exercise.

Method: One recreationally well-trained (not elite) man subject (26 yrs, $80 \mathrm{~kg}, 187 \mathrm{~cm}$, VO2max: $64.8 \mathrm{ml} / \mathrm{O} 2 / \mathrm{kg}, 315 \mathrm{~W}$ at $3 \mathrm{mmol} / \mathrm{L}$ ) cycled $692 \mathrm{~km}$ in non-stop exercise from Freiburg (D) to Koper (SLO) with difference in altitude of $2700 \mathrm{~m}$. The assessment of knee extensor neuromuscular parameters began with evaluation of central fatigue, peak torque during Maximal Voluntary Contraction (MVC) was recorded and interpolated twitch method (superimposed double twitch over MVC delivered by electrical stimulus via femoral nerve) was used to evaluate Voluntary Activation level (VA). The peripheral fatigue was assessed by recording peak torque during electrical stimulation at low and high frequency ( $20 \mathrm{~Hz}$ and $80 \mathrm{~Hz}$ respectively, $0.5 \mathrm{~s}$ long) in relaxed muscle state via femoral nerve. 
Results: Drop in power output (PO) was observed, despite low intensity was performed along $28 \mathrm{~h} 23 \mathrm{~m}$ without rest (HR: $120.7 \mathrm{bpm}, 67 \%$ HRmax, PO: $177.7 \mathrm{~W}, 49 \%$ POmax and VO2max: $2.360 \mathrm{l} / \mathrm{m}, 45 \%$ VO2max). We observed a decrease in MVC $(320.9 \mathrm{~N} / \mathrm{m},-3.62 \%$, absolute and relative data respectively) soon after the exercise. VA level increased a little after the cycling event $(87.08 \%,+9.52 \%)$ and the torque decreased at low frequency $(207 \mathrm{~N} / \mathrm{m},-14.76 \%)$ and decreased at high frequency $(352.92 \mathrm{~N} / \mathrm{m},-1.7 \%)$.

Discussion: The lack of data does not allow making any reliable comparison with this case study. To date only one paper of longlasting exercise (five hours of cycling) is available (1) concerning neuromuscular impairments. The small difference $(-1.7 \%)$ it could be neglect the high frequency fatigue. The muscle impairment compound at low frequency stimulus direct to state low frequency fatigue (-14.76). Unexpectedly the VA level $(+9.52 \%)$ increased soon after UE, the MVC decreases a little $(-3.62 \%)$. An overview of the data presented, lead to retain that an additional motor drive it could be verified in order to compensate the peripheral impairments. Concluding, the data suggest the neurophysiological impairments cannot explain entirely the drop in performance (power output), accordingly the motivational state and energy supply could have been influenced the UE cycling performance (2).

\section{References}

1. Leper R. 2002 doi:10.1152/japplphysiol.00880.2001

2. Marcora 2010 EJAP doi 10.1007/s00421-01 0-1418-6

\section{Session 4. Physical Activity \& Health}

\author{
Walking training effects on DHEA-S and TNF- , \\ in post-menopause: role of $\mathrm{ACE} \mathrm{I} / \mathrm{D}$ polymorphism
}

\author{
P. Izzicupo ${ }^{1}$, M. A. D'Amico' ${ }^{1}$, A. Di Fonso ${ }^{1}$, A. Bascelli' ${ }^{1}$, S. Gallina ${ }^{2}$, \\ A. Di Baldassarrel
}

${ }^{1}$ Dept Medicine and Aging Sciences, University of Chieti-Pescara, Italy ${ }^{2}$ Dept Neurosciences and imaging, University of Chieti-Pescara, Italy

Introduction: Angiotensin-converting-enzyme (ACE) insertion/deletion (I/D) polymorphism was suggested to influence inflammatory cytokine levels as well as adrenal androgens production. Post-menopausal women are characterized by both a systemic chronic inflammation and a decline of Dehydroepiandrosterone sulfate (DHEA-S) levels. Recently, several studies have highlighted the effects of physical activity on the immune system and adrenal activity, with active people showing lower levels of inflammatory markers and higher levels of DHEA-S.

The aim of this study was to investigate the effect of walking training (WT) in post-menopausal women on both plasma DHEA-S and tumor necrosis factor alpha (TNF- ) levels among different ACE I/D genotype. Methods: Thirty-two sedentary post-menopausal women $(55.9 \pm 4.2$ years) were enrolled in the study. Before and after 3 months of WT at moderate intensity (40-50 min; 4 days/week), they were evaluated for TNF- and DHEA-S plasma levels. The ACE I/D polymorphism was analyzed by polymerase chain reaction.

Results: On the bases of the I allele presence, subjects were divided in two genetic groups (DD: n 16; ID/II: n 16). Before the intervention program, ACE DD genotype showed lower DHEA-S plasma concentration $(\mathrm{P}=0.03)$ as well as a tendency to higher levels of TNF- $\alpha$ respect to ID/II individuals. The WT program induced in both genetic groups an increase of DHEA-S and a decrease of TNF- $\alpha$ plasma concentration $(\mathrm{P}=0.005, \mathrm{P}=0.01$, respectively), independently on $\mathrm{ACE} \mathrm{I} / \mathrm{D}$ polymorphism. However, there were differences between the genetic variants, I allele carriers being characterized by higher DHEA-S and lower TNF- $\alpha$ plasma levels $(\mathrm{P}=0.03, \mathrm{P}=0.05$, respectively).
Conclusion: ACE I/D polymorphism influences both DHEA-S and TNF- $\alpha$ levels in both basal condition and after an aerobic training at moderate intensity, with I carriers showing a more favourable adrenal activity level and systemic inflammatory profile. The introduction of an exercise program positively influences the menopause immune-neuroendocrine alterations independently of ACE I/D genotype.

\section{Core strength synergy and its influence in non chronic LBP} A. R. Calavalle, D. Sisti ${ }^{1}$, G. Andolina
, M. Gervasi
M. C. C. Spineto

${ }^{1}$ Dipartimento di Scienze Biomolecolari, Sezione di Scienze Motorie e della Salute, Università degli Studi di Urbino "Carlo Bo",

Urbino (PU), Italia

Introduction: LBP is often associated to the job related activities and the lack of core strength. Firefighters have a high percentage of risk in job related LBP, such as many other kind of job. The purpose of this study was to evaluate the influence of the core muscles strength in non chronic LBP. Methods: 18 male firefighters (age 42,9 $\pm 7,4$; BMI 25,6 $\pm 3,0$ ) from the Urbino Fire Department, PU (Italy) were tested by the Roland Morris Questionnaire (RMQ) and a core stability test. The RMQ test consisted in 24 items to describe the influence of LBP on your own day life activities. The core stability tests consisted in four different static plank positions, subjects' whole body on a straight line, rested on their elbows: front, reverse and both sides positions. The time was recorded at the moment the subjects lost his best position. Data were analyzed using multiple linear regression analysis. Predictor variables were: duration of plank test in front, reverse, sides positions and ratio between frontal and reverse value. Dependent variable was Roland Morris questionnaire score.

Results: data reported a positive correlation between the RMQ values and the ratio front/reverse core stability tests values $(p=0,02)$. Interestingly there were no significantly correlation comparing RMQ ant the core tests separately.

Discussion: This abstract shows that as ratio between reverse plank and front plank rises in favour of the former ones, a rise in RMQ scoring is obtained. The firefighters that had a lack of synergy in the trunk muscles (front $v s$ reverse), had a LBP influences in their day life activities. In conclusion, a simple method aimed at checking LBP risk has been proposed; larger sample will be necessary to validate the proposed method.

Aerobic and resistance exercise have different short-term effects on glucose levels in type 2 diabetes subjects

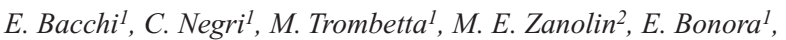 \\ M. Lanza ${ }^{3}$, P. Moghetti ${ }^{1}$
}

${ }^{1}$ Section of Endocrinology and Metabolism, Department of Medicine, Azienda Ospedaliera Integrata Universitaria and University of Verona, Verona, Italy

${ }^{2}$ Department of Public Health and Community Medicine, University of Verona, Verona, Italy

${ }^{3}$ School of Sports and Exercise Sciences, Department of Neurological, Neuropsychological, Morphological and Movement Sciences,

University of Verona, Verona, Italy

Aerobic (AER) and Resistance (RES) exercise similarly improve HbAlc levels in type 2 diabetes subjects. However, it is still unknown whether exercise-induced acute changes in blood glucose differ according to exercise type. Our aim was to compare glucose level changes after a single 
bout of AER or RES exercise in trained diabetic subjects.

Twenty-five subjects participating in the RAED2 Study, a RCT designed to compare the effects of AER and RES training in diabetic patients, were submitted to a continuous glucose monitoring system during a 60min exercise and the following 47-h. Glucose concentration areas under the curve (AUC) during exercise, the subsequent night, and the 24-h period following the exercise, as well as the corresponding periods of the non-exercise day, and the low (LBGI) and high (HBGI) blood glucose indices, which summarize duration and extent of hypoglycaemia or hyperglycaemia, respectively, were assessed.

AER and RES groups showed similar HbAlc improvements after training. However, comparison of glucose AUC during the 60-min exercise and the corresponding period of the non-exercise day showed different behaviors between groups (time-by-group interaction, $\mathrm{p}=0.04$ ), glucose being lower during exercise in the AER but not in the RES group. Similar differences between groups were observed in the comparison of nocturnal periods of exercise and non-exercise days ( $\mathrm{p}=0.02$ ). Consistently, in the AER group nocturnal LBGI was higher in the exercise day than in the non-exercise day $(\mathrm{p}=0.012)$, whereas there were no differences in the RES group.

Although AER and RES training show similar long-term metabolic effects in diabetic subjects, the short-term effects of single bouts of these exercise types differ, with a potential increase in risk of late-onset hypoglycaemia after AER exercise. These findings suggest that, in diabetic subjects, medications and diet adjustments to exercise should take into account the scheduled type of physical activity.

\section{Heart rate alteration during a constant load exercise} in the different menstrual phases

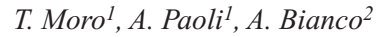 \\ ${ }^{1}$ Dip. Scienze Biomediche, Università di Padova, Italia ${ }^{2}$ DISMOT - Università di Palermo, Italia}

In fitness centres, the most common way to modulate exercise's intensity is heart rate (HR) control and therefore training at constant heart rate (CHR) is widely used but this method assume that exercise's response to exercise should be always the same; Instead there are conflicting results about menstrual cycle's effects on physiological response to exercise (Jense de Jonge 2003). Many researches have investigated the influences of estrogenic hormone levels in sport performance (Sarwar 2006; Phililps 1996), muscle strength and heart rate during maximal and submaximal exercise (Oosthuyse 2005; Kishali 2006). No study has attempt to verify if CHR correspond to a constant load of endurance exercise throughout the 28 days of menstrual cycle and therefore if this method could be suitable to right size training stimulus also in the different phases of menstrual cycle.

20 women $(25 \pm 8,66$ years old an $56,1 \pm 8,82 \mathrm{~kg})$ was tested at cycloergometer in each phases of menstrual cycle (follicular (FOL) days 1-13, ovulation (OV) days 14-15, luteal (LUT) days 15-21, menstruation (MES) days 21-28); subjects didn't take oral contraceptive during the experiment period. Cycle phases were preliminary confirmed by temperature and LH urine concentration measurement (Lebrun 2003). HR, blood lactated and RPE value was collected and analysed during every training session (FOL, LUT, OV, MES).

The results showed a significantly lower HR during the MES phase compared to the other phases whilst there were no significant changes in other variables: blood lactate and RPE values, confirming the steadfastness of the internal load.

These results suggest that ovarian hormones variation during menstrual cycle could influence heart response to constant load endurance exercise. In conclusion our data suggests that CHR could underestimate exertion during the menstrual phase therefore female subjects would be forced to increase the external load to maintain the same (supposed) relative training intensity.

References

- Janse de Jonge XA. Sports Med. 2003;33(11):833-51

- Sarwar R et al. J Physiol. 1996 May 15;493 ( Pt 1):267-72

- Philips SK et al. J Physiol. 1996 Oct 15;496 ( Pt 2):551-7

- Oosthuyse T et al. Eur J Appl Physiol. 2005 Jun;94(3):268-76

- Kishali NF et al. Int J Neurosci. 2006 Dec;116(12):1549-63

- Lebrun CM et al. Br J Sports Med. 2003 Aug;37(4):315-20

\section{Successful treatment of muscle tone in facial paralysis: a multidisciplinary study}

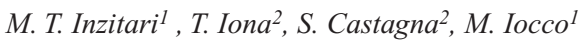 \\ ${ }^{1}$ Department of Physical Medicine and Rehabilitation -University of Magna Graecia, Catanzaro, Italy \\ ${ }^{2}$ School of Medicine, University of Magna Graecia, Catanzaro, Italy}

Introduction: Communication through language is a noble and peculiar ability of the human genre, necessary to make individuals feel part of a social context. This ability can be seriously compromised by the persistent deficit due to a facial lesion resulting from a concussion or a tumor extirpation. The purpose of this study is to re-establish the balance of orofacial muscle tone by training of the fonoarticulatory functions of tongue and stimolations of anterior and posterior neck muscles in order to give to an individual with facial paralysis a normal social life.

Methods: 21 subjects were enrolled (mean 48,9 years; 6 males and 15 females).

For all subject EMG Biofeedback were measured at the start and end of the treatment and the assessment of the force muscle according to the classification MRC (Medical Research Council) and was used the evaluation scale FIM (Functional Independent Measure) to know the severity of disability. Training program (five 40-min sessions per week-1, for three months) included proprioceptive exercise, postural and strength orofacial and chest muscular exercise.

Results: $95 \%$ of treatments showed increasing of orofacial muscle tone balance (MRC $\geq 3$ ), and a consequent decrease of the FIM . 12 subjects $(63 \%)$ achieved functional recovery in the short time, while $36.8 \%$ of the subjects needed more time.

Conclusion: Despite different clinical situations, data show a functional and psycosocial trend enough satisfactory. This suggests the need to work with a global medical and trainer team in order to increase the speed of functional recovery of subjects.

\section{References.}

- Migne R., Diagnostic at traitmement des doulores communes d'origine rachidenne, Parigi, Espansion Scientifique Francaise, 1989

- Granger C.V., Perrucca L., Francchioni F.P., Battaglia M.A., Russell C.F., The FIM Instrumental in United States and Italy, Am J. Phys.Med.Rehabil., 2002

- Cristofaro M.G., Inzitari M.T., Riabilitazione delle funzioni compromesse dei muscoli mimici, Ed.Marrapese, 2007

Validation of a method for symmetry line detection: the analysis of posture by means of a geometric method for the determination of the symmetry line in the vertebral column

\section{Di Angelo ${ }^{\text {, }}$ P. Di Stefano Paolo ${ }^{1}$, P. Raimondi ${ }^{2}$, A. Di Giulio ${ }^{2}$, M.G. Vinciguerra ${ }^{2}$}

${ }^{1}$ Dept. of Industrial and Information engineering and Economics, University of L'Aquila 
${ }^{2}$ Dept. of Biotechnological and Applied Clinical Sciences, University of L'Aquila

In this work a new method for symmetry line recognition, from 3D scanned data of a subject's back, is presented. The new method is validated by comparison with traditional techniques based on cutaneous marking. For this purpose, the upright standing and sitting postures of a sample of 75 subjects, who usually perform different sports activities, are analysed. Error in symmetry line detection is measured as the distance between the estimated symmetry line and the position of the markers. The proposed method is compared with another one described in literature which has been validated in clinical field. Results are analysed and critically discussed.

\section{Increases of bone density in cART-treated persons after 12 weeks} of brisk walking

\section{Bonato ${ }^{\text {, S. Sossolasco }}$, L. Galli ${ }^{2}$, G. Pavei ${ }^{3}$, G. Merati $^{3}$, A. La Torre', P. Cinque ${ }^{2}$}

${ }^{1}$ Department of Biomedical Sciences for Health, University of Milan, Italy

${ }^{2}$ Department of Infectious Diseases, San Raffaele Scientific Institute, Milan, Italy

${ }^{3}$ Human Physiology Department, University of Milan, Italy

Moderate intensity aerobic activity has a potential in preventing bone loss in general population. Aim of this study was to evaluate the effects of brisk walking, with or without strength exercise, on bone mineral density in HIV-infected treated persons.

Twenty-eight HIV-infected, cART-treated, sedentary subjects with $\mathrm{VL}<50 \mathrm{c} / \mathrm{mL}$ were enrolled in a 12 -week outdoor training protocol, consisting of 3 sessions/week of $60 \mathrm{~min}$ walking at $67-70 \%$ of HR (Heart Rate) $\max \pm 30$ min circuit training at $65 \%$ of $1-\mathrm{RM}$ (Repetition Maximum). Subjects were examined at baseline (BL) and 12 weeks (W12) by 6-minute walking test (6MWT); 1-RM Test; maximal number of crunches in $30 \mathrm{sec}$ and by dual energy X-ray absorptiometry (DEXA) to evaluate lumbar spine and femoral bone mineral density, and t- and zscores - in addition to morphometric (BMI, waist, hip and leg circumference) and blood examination (cytometry, fasting total, HDL and LDL cholesterol, triglycerides, glucose, insulin; AST/ALT, ALP, gGT, creatinine, CPK, HbA1c; CD4+ and CD8+, plasma HIV-RNA). Differences over time were tested by Wilcoxon-signed rank test and between groups by Mann-Whitney test; results are expressed as [median (Q1-Q3)].

Twenty-seven (96\%) participants [19M, 8F; age 48 (43-54) yrs.; CD4+ 624 $(478-708) / \mu \mathrm{L}$; ART with PI: 13 patients, with NNRTI: 7 patients, and including TDF: 15 patients] completed the outdoor 12-week training program with a median adherence of $61 \%(50-70 \%)$ : 18 in the "walk" group and 9 in the "walk and strength" group. At W12, participants showed significant improvement of distance by 6MWT [658 (605-691) $\mathrm{m}$ vs 715 $(690-830) \mathrm{m},<0.0001]$, and of performance in all strength exercises (crunch $\mathrm{p}=0.023$, lat machine $\mathrm{p}=0.016$, chest press $\mathrm{p}=\mathrm{p}=0.016$, leg extension $\mathrm{p}=0.016$, sitting calf $\mathrm{p}=0.008$, leg press $\mathrm{p}=0.016$ ). DEXA spine L1-L4 (z-score) improved significantly in the whole group [-1.15 (-1.7/ - 0.7$)$ vs $-1.05(-1.4 /-0.3), \mathrm{p}=0.002]$, and femoral neck [-0.8 (-1.4 / -0.2) vs $-0.5(-$ $1.2 /-0.2), \mathrm{p}=0.047]$ femoral ward $[-1(-1.9 /-0.6)$ vs $-0.75(-1.4 /-0.2)$, $\mathrm{p}=0.004] \mathrm{z}$-scores in the "walk" only group. There was no z-score difference at BL between patients with/out PIs, NNRTIs or TDF. However, spine $\mathrm{z}$-score improved significantly in patients receiving TDF [-1.4 (-1.7 / -0.7) vs $-1.3(-1.55 /-0.5), \mathrm{p}=0.002]$. At W12 BMI [25.7 (24.6-26.3) $\mathrm{kg} / \mathrm{m} 2$ vs $24.9(21.4-26) \mathrm{kg} / \mathrm{m} 2$ ], waist circumference [93 (87-100) $\mathrm{cm} v s$ $92(85.5-98.5) \mathrm{cm}, \mathrm{p}=0.029)$ and LDL [126 (113-154.5) mg/dL vs $116(96-$
137.5) $\mathrm{mg} / \mathrm{dL}$ ] were also significantly improved in the whole group, whereas no significant changes were observed for the other variables, The above 12-week program improved fitness and bone density in HIVinfected treated subjects, in addition to some morphometric variables and serum LDL. Brisk walking, with or without strength exercise, might help control the long-term consequences of cART.

\section{Evaluation spatio-temporal gait parameters with and without a maternity support for the treatment of low back pain in pregnancy}

\section{A. Quirino Lucciola ${ }^{1}$, C. Cortis ${ }^{1}$, D. Lacitignola ${ }^{2}$, P. Mondini ${ }^{3}$, A. Rodio ${ }^{1}$}

${ }^{1}$ Department of Human Sciences, Society and Health, University of Cassino and Southern Lazio, Italy

${ }^{2}$ Department of Electrical and Information Engineering, University of Cassino and Southern Lazio, Italy

${ }^{3}$ ErgonixART s.r.l. - San Cesareo (RM), Italy

Introduction: The numerous physical and hormonal changes occurring during pregnancy may lead to an altered postural balance causing an increased frequency of musculoskeletal pain at low back level and the risk of falling. Thus, the purpose of this study was to evaluate if an innovative bodysuit (ComfortBody $(\mathrm{C})$ designed to reorganize the augmented load from the lumbar area to the entire back, would result in spatio-temporal gait parameters changes during walking when compared to a placebo bodysuit in pregnant women.

Methods: After given their written consent, 13 pregnant women (age: $31.7 \pm 3.4$ years; weight gain: $10.5 \pm 2.5 \mathrm{~kg}$ ) in their second and third trimester of pregnancy took part in a single-blind, randomized, placebocontrolled, crossover study. Participants were asked to walk 10-min on a treadmill (speed: $3 \mathrm{~km} / \mathrm{h}$; slope: $0 \%$ ) during four experimental sessions (familiarization-F, wearing the ComfortBody@-B, placebo-P, or without support-WS) with a 30-min recovery between sessions. Experimental sessions were assessed on sessions 2 and $3(\mathrm{C}$ and $\mathrm{P})$ through permutedblock randomization. Session 4 (WS) was optional and used to assess neuromuscular adaptation of the specific motor task (walking on a treadmill). Computerized stride analysis was used to measure the spatial (stride length) and temporal (stride duration, single and double-limb support duration as measured as \% of gait cycle) parameters of the walking pattern. ANOVA for repeated measures was used to assess differences $(0.05)$ in experimental sessions.

Results: B and P experimental conditions showed no significant differences in stride length (B: $1.12 \pm 0.06 \mathrm{~m} ; \mathrm{P}: 1.11 \pm 0.07 \mathrm{~m}$ ), stride duration (B: $1.34 \pm 0.08 \mathrm{~s} ;$ P: $1.34 \pm 0.08 \mathrm{~s}$ ), single-limb (B: $65.8 \pm 5.5 \%$; P: 66.5 $\pm 5.4 \%$ ) and double-limb (B: $34.2 \pm 5.5 \%$; P: $33.5 \pm 5.4 \%$ ) support. On the other hand, significant $(\mathrm{p}<0.05)$ differences emerged between $\mathrm{F}$ and WS conditions in stride length (FS: $1.10 \pm 0.07 \mathrm{~m}$; WS: $1.13 \pm 0.07 \mathrm{~m}$ ), stride duration (FS: $1.32 \pm 0.09 \mathrm{~s}$; WS: $1.36 \pm 0.08 \mathrm{~s}$ ), single-limb (FS: $66.7 \pm 5.3 \%$; WS: $65.0 \pm 4.9 \%$ ) and double-limb (FS: $33.3 \pm 5.3 \%$; WS: $34.9 \pm 4.9 \%$ ) support.

Discussion and conclusion: Results indicate that ComfortBody $₫$ might not induce significant differences in the spatio-temporal gait. However, data seem to show a tendency to increase the double-limb support and decrease the single-limb support duration when wearing the body compared to the placebo condition. Investigations are hence in progress to support our speculation regarding the stabilizing effect of the ComfortBody $₫$ on walking activities. The amount of time spent in double-limb support may in fact be a useful indicator of balance control during walking (1), probably reducing the risk of fall in pregnant women, while the reduction in time of single support could reduce the muscular effort caused by weight gain. 


\section{Survey on the eating habits of high-level gymnasts practicing rhythmic gymnastics}

\author{
G. Berlutti ${ }^{1}$, A. Tinto ${ }^{1,2}$, M. Micheletti Cremasco ${ }^{2,3}$, M. $_{\text {Piazza }}{ }^{1,4}$ \\ ${ }^{1}$ Italian Gymnastics Federation \\ ${ }^{2}$ S.U.I.S.M. - School of Physical and Sports Education - University \\ of Turin \\ ${ }^{3}$ Department of Life Sciences and Systems Biology - University of \\ Turin \\ ${ }^{4}$ Department of Anatomy, Histology and Forensic Medicine, University \\ of Florence
}

Introduction: The research was carried out during the course of the XXIV European Rhythmic Gymnastics Championships in Turin in 2008. The initiative was proposed and implemented by the Federazione Ginnastica d'Italia, the work was conducted jointly by the medical and the technical staff composed of federal coaches as well as professors at Italian university schools of motor sciences. The research was led by Dr. Giovanna Berlutti, the doctor in charge of the national gymnastics team of FGI.

Aim: The aim of this research is to perform a survey on the eating habits of high-level gymnasts practicing Rhythmic Gymnastics.

Methods: A group of 139 gymnasts were asked to take the survey consisting of 35 essay questions, prepared in three languages (Italian, English and Russian) under the assistance of multilingual tutors. The initial (7) questions addressed the sports they practiced. Then 3 questions regarded their menstrual cycles. And 17 specific questions were asked concerning the diet applied by the gymnasts.

Results: The results of the survey were stored on a database and analyzed. Only $28.6 \%$ of the gymnasts analyzed answered yes to the question "Do you follow a nutritional regimen?". Only a small part (7.1\%) follows a regimen under medical observation. The remainder follows regimens as advised by coaches or instructors. It was found that $93 . \%$ of the gymnasts have regular breakfast. A slightly lower percentage always has lunch and supper. Percentages drop significantly as to how many of them have mid-morning snacks, i.e. only $15 \%$ of the gymnasts consume them regularly. Fewer gymnasts $(11.5 \%)$ consume afternoon snacks and even fewer $(4.3 \%)$ have evening snacks. Water intake during work-outs is fairly proper ( $95 \%$ said yes). $28.8 \%$ takes fluid-electrolyte and/or vitamin and/or energy supplements every day, whereas $15 \%$ of the gymnasts uses such supplements twice or three times a week. It turns out that ice cream is a rat her popular food, and it is consumed by the athletes as dessert after meals (57\%) whereas some of them eat ice cream instead of lunch and others eat ice cream as snack.

Conclusions: The data represent rather correct eating habits, even if the daily requirements could be met by consuming foods by distributing them into more frequent meals throughout the day without the need to eat them at the major meals of the day necessarily. The data confirm fully that excessive thinness is absolutely not necessary to practice this discipline at high levels. The results accompanying this survey confirm this affirmation.

\section{Symptomatic and asymptomatic overuse injuries in recreational golf players}

\author{
S. Bellagamba ${ }^{1}$, F. Lucertini ${ }^{2}$, A. Federici ${ }^{2}$, K. Quagliani ${ }^{1}$, M. Rinaldi ${ }^{2}$, \\ C. Bartolucci ${ }^{1}$ \\ ${ }^{1}$ Faculty of Sport and Exercise Science, University of Urbino \\ "Carlo Bo", Urbino (PU), Italy \\ ${ }^{2}$ Department of Biomolecular Sciences, Division of Exercise \\ and Health Sciences, University of Urbino "Carlo Bo", Urbino (PU), \\ Italy
}

Golf is classified as a physical activity of low-to-moderate intensity with a metabolic cost ranging from 2.5 to 6 METs, thus suitable for most people. However, as many other sports, and particularly those whose fundamentals need asymmetric movements - such as the 'swing' - golf leads to a high incidence of overuse injuries of the muscle-skeletal system in middle and high-level players. Although this injuries may be clinically significant, sometimes they are asymptomatic, thus leading to a probable 'hidden' worsening of the clinical picture. Among golf players, this is particularly true for what concern hand injuries.

In order to evaluate the incidence of symptomatic and asymptomatic overuse injuries, 60 male adults $(53.2 \pm 12.1 \mathrm{y})$ recreational golf players underwent a face-to-face questionnaire and a sonographic assessment. The questionnaire was concerned about both golf practice/training - how many years, average weekly frequency, and single session duration - and injuries status - symptoms, pain localization (by means of the Pain Drawing) and intensity (by means of the Visual Analog Scale, VAS). Acute injuries and those clearly not related with golf practice were not retained for later descriptive analysis. Sonographies of the elbow, wrist, and hand of both arms of each player were made by means of a portable ecocolor powerdoppler sonographer with 12 to $18 \mathrm{MHz}$ linear transducers. Results were classified in 3 categories of years of practice ( 0 to 5,6 to $10,>10 \mathrm{yrs})$ and monthly training hours (no training, $<8, \geq 8 \mathrm{~h} / \mathrm{mo}$ ).

Pain drawing results evidenced that only $38 \%$ of the recreational golf players did not report any pain symptom, whereas the remaining symptomatic $62 \%$ suffered from low back pain (34\%) and felt pain in the elbow $(12 \%)$, shoulder $(8 \%)$, hand (3\%), and 'other' (5\%) regions (hip, lower limb, knee, foot). VAS results, on average, evidenced values ranging from $4.1 \pm 1.6$ (shoulder) to $5.3 \pm 1.4$ (elbow). Sonographies confirmed both wrist and elbow synovial expansions in all the symptomatic players reporting pain in those regions. Sonographies of the asymptomatic players revealed that about $50 \%$ were actually not injured, whereas, of the remaining players, $46 \%$ suffered from synovial thickening and palm fibrosis (hand), 18\% from tendon cysts and fluid films (wrist), and $36 \%$ from synovial thickening, epitrocleitis and epicondylitis (elbow).

On average, as long as of golf practice years increase, elbow and low back pain occurrences rose, while shoulder and 'no pain' occurrences decreased. As for training monthly time, average pain occurrences rose in elbow, shoulder, and low back regions, as well as 'no pain' reports. Average VAS results seemed to be not affected neither by golf practice years nor by training monthly time.

In conclusion, recreational golf players may be at risk of symptomatic and asymptomatic overuse injuries, particularly in the low back, elbow and hand areas, and years of practice may increase the incidence of those overuse injuries. 


\section{ORAL PRESENTATIONS}

\section{Saturday, October $6^{\text {th }}, 2012$}

\section{Session 5.1. Functional Adaptation to Exercise}

\author{
Influence of skeletal age on anthropometric characteristics \\ and performance in young soccer players
}

\author{
R. Modena ${ }^{1}$, A. Brighenti ${ }^{1}$, A. Savoldelli ${ }^{1}$, B. Pellegrini ${ }^{1,2}$, F. Schena ${ }^{1,2}$ \\ ${ }^{1}$ CeRiSM, Research Center of Sport Mountain and Health, University \\ of Verona, Rovereto, Italy \\ ${ }^{2}$ Department of Neurological, Neuropsychological, Morphological \\ and Movement Sciences, University of Verona, Italy
}

Background: Several studies showed that physical performance is related with biological maturation in young athletes and this relationship may be stronger from 13 years (1).

The estimate of biological maturation in sport was usually performed by indirect methods such as evaluation of sexual maturation or by using $\mathrm{x}$ rays. To our knowledge no study has used ultrasound methods to estimate skeletal age in youth soccer players.

Aim: This study proposes to investigate the effect of skeletal age, assessed by ultrasound, on physical performance and anthropometric characteristics of young soccer players. The relationships between anthropometric characteristics and performance on one side and skeletal (SA) and chronological age (CA) on the other side were studied in 58 young soccer players of an Italian professional team, divided in four categories (Under 16 (U16), n=11; Under 14 (U14), n=12; Under 13 (U13), $n=19$ and Under 10 (U10), $\mathrm{n}=16$ ).

Methods: We evaluated SA of players by quantitative ultrasonography of the proximal phalanges of the hand using a sonograph (DBM Sonic BP IGEA) (2). We also assessed two important parameters related to soccer performance: muscular explosive power evaluated by means of one-leg vertical jump height (OLVJ) and specific aerobic capacity evaluated taking into account the distance covered during the Yo-Yo Intermittent Recovery Test Level 1 (YYIRT1) (3).

Results: The weight and height were correlated with SA in U14 $(\mathrm{r} 2=0.69, \mathrm{p}<0.01$ and $\mathrm{r} 2=0.55, \mathrm{p}<0.01$, respectively), U13 ( $\mathrm{r} 2=0.36$, $\mathrm{p}<0.01$ and $\mathrm{r} 2=0.3, \mathrm{p}<0.05)$ and $\mathrm{U} 10(\mathrm{r} 2=0.46, \mathrm{p}<0.01$ and $\mathrm{r} 2=0.37$, $\mathrm{p}<0.05)$. The BMI was correlated with SA in $\mathrm{U} 14(\mathrm{r} 2=0.48, \mathrm{p}<0.01)$ and $\mathrm{U} 13(\mathrm{r} 2=0.26, \mathrm{p}<0.05)$.

Just the height was weakly related with CA in $\mathrm{U} 13(\mathrm{r} 2=0.23, \mathrm{p}<0.05)$ and $\mathrm{U} 10(\mathrm{r} 2=0.22, \mathrm{p}=0.06)$.

The SA was correlated with OLVJ in three categories (U16 r2 $=0.4$, $\mathrm{p}<0.05$; $\mathrm{U} 14 \mathrm{r} 2=0.48, \mathrm{p}<0.05$; $\mathrm{U} 13 \mathrm{r} 2=0.53, \mathrm{p}<0.01$ ) but not in younger players, while no correlation was found between CA and OLVJ. Neither CA nor SA were correlated with distance covered in YYIRT1.

Discussion and Conclusion: Results showed that the SA is more related with the anthropometric characteristics than with CA in soccer players, before the age of the puberty.

Furthermore the SA is more useful then CA to compare the results in OLVJ except in 10-years old players.

The performance in YYIRT1 doesn't seem to related neither by SA nor by CA.

The assessment of anthropometric characteristics and OLVJ in young soccer players is less effective and accurate if related to CA than SA.

References

1. Carling C. et al. Journal Sports of Sciences, 2011, 1-11

2. Vignolo M. et al. The Open Bone Journal, 2010, 2, 38-42

3. Kustrup P. et al. Med Sci Sports Exerc. 2003;35(4):697-705
Effect of different impact loading sports on bone quality and body composition in pre-pubertal girls

C. Milanese ${ }^{1,2}$, F. Piscitelli $^{1,2}$, V. $^{\text {Cavedon }}{ }^{1,2}$, C. Zancanaro $^{1,2}$

${ }^{1}$ Dip di Scienze Neurologiche, Neuropsicologiche, Morfologiche e Motorie, University of Verona, Italy

${ }^{2}$ Faculty of Motor Sciences, University of Verona, Italy

Adaptations of bone to exercise are dependent in part on the magnitude and type of loading stimulus and the timing of the loading exposure across the life cycle. Women's artistic gymnastics and volleyball are Olympic disciplines practiced by millions worldwide, which are characterized by high impact loading (3-6 times the body weight) and muscle strength requirement. The aim of this study was to compare the effect of different impact loading sports on bone quality and body composition of pre-menarcheal girls. A total of 30 pre-pre-menarcheal athletes (aged $11.1 \pm 1.33$ [SD]y) participating in volleyball $(\mathrm{n}=10)$ and artistic gymnastics at high (HGYM, n=10) and low (LGYM, n=10) intensity training underwent total body DXA analysis. Output measurements were bone mineral content (BMC), areal bone mineral density (aBMD), fat free soft tissue mass (FFST), fat mass (FM), and percentage FM at the total body and regional level (arms, legs and trunk) as well as specific axial skeleton sites (thoracic and lumbar spine, pelvis). After adjusting for body size, total body composition measurements were all significantly $(p<0.01)$ different in the three groups of pre- pubertal athletes. VOLLEY showed lower BMC and FFST, and greater FM and \%FM vs both HGYM and LGYM. The main difference between LGYM and HGYM was greater $\% \mathrm{FM}$ in the former. Similar results were found at the regional level (but for similar BMC in the legs) and at lumbar spine and pelvis. Linear regression analysis showed that a model using FFST mass the as independent variable is able to explain more than $90 \%$ of variance in total body less head BMC in the whole group of impact loading athletes, whereas introducing \%FM in the model did not improve it predictive power at all. The findings of this study provide evidence that greater bone mineral accrual takes place in pre-menarcheal impact loading athletes participating in artistic gymnastics $v s$ volleyball and the dose of impact loading activity mainly affect $\% \mathrm{FM}$; moreover, results suggest that $\mathrm{BMC}$ in the weight-bearing skeleton is strongly affected by FFST.

\section{Cardiorespiratory fitness impairment in polycystic ovary} syndrome

\section{E. Bacchi ${ }^{1}$, C. Negri ${ }^{1}$, D. Di Sarra ${ }^{1}$, F. Zambotti ${ }^{1}$, M. Dall'Alda ${ }^{1}$, F. Tosi ${ }^{1}$, F. Schena ${ }^{2}$, P. Moghetti ${ }^{1}$}

${ }^{1}$ Section of Endocrinology and Metabolism, Department of Medicine, Azienda Ospedaliera Integrata Universitaria and University of Verona, Verona, Italy

${ }^{2}$ School of Sports and Exercise Sciences, Department of Neurological, Neuropsychological, Morphological and Movement Sciences,

University of Verona, Verona, Italy

Polycystic Ovary Syndrome (PCOS) is a common endocrine disorder characterized by chronic anovulation, hyperandrogenism and frequent insulin resistance. Preliminary data have shown that subjects with PCOS have a reduced cardiopulmonary capacity. This study aims at evaluating cardiorespiratory fitness in PCOS compared with healthy subjects. Twenty-seven PCOS women (mean \pm SD: age $23.0 \pm 4.1 \mathrm{yr}$, BMI $24.8 \pm 4.5$

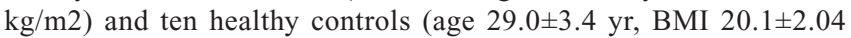
$\mathrm{kg} / \mathrm{m} 2$ ) were enrolled. We assessed anthropometric features, hormonal and metabolic parameters and insulin sensitivity (by glucose clamp technique). Cardiorespiratory fitness was assessed by a maximal exer- 
cise testing (ramp protocol of $15 \mathrm{Watt} / \mathrm{min}$ ) to evaluate peak oxygen consumption (VO2peak), oxygen uptake at aerobic threshold, and the maximal workload. BMI, waist circumference, testosterone were higher in PCOS subjects compared with healthy women, while insulin sensitivity was significantly reduced in PCOS women. VO2peak and maximal workload were also significantly reduced in PCOS women $\left(25.6 \pm 5.3\right.$ vs $35.4 \pm 5.4$ mLâ ${ }^{\top}{ }^{\mathrm{TM}} \mathrm{kg}-1 \hat{\mathrm{a}}^{\wedge} \mathrm{TM} \min -1, \mathrm{p}<0.0001 ; 136 \pm 21 v s$ $168 \pm 37$ Watt, $\mathrm{p}=0.002$, respectively). Similarly, oxygen consumption at aerobic threshold was reduced in PCOS subjects. Similar results were obtained when normal weight PCOS women $(n=13)$ were compared with normal weight healthy subjects $(n=10)$. In the entire cohort of subjects, VO2peak was negatively associated with BMI, testosterone, fat mass, insulin levels and positively associated with insulin sensitivity. In multivariate models, VO2peak was independently predicted by PCOS diagnosis, fat mass and insulin levels $(\mathrm{p}<0.0001, \mathrm{R} 2=0.68)$. In conclusion, our data show that PCOS subjects have a striking alteration of cardiorespiratory fitness. This abnormality is associated with PCOS status independently of insulin resistance and obesity.

\section{Relationship between dynamic balance, as measured by the Eight} Balance Test, BMI and age, in the middle childhood

\section{F. Magno ${ }^{1}$, G. Bardaglio', D. Marasso ${ }^{1}$, G. Musella ${ }^{1}$, E. Rabaglietti ${ }^{1,2}$,} S. Ciairano ${ }^{1,2}$

${ }^{I}$ Motor Science Research Centre, Research Unit Motor Skills and Development, SUISM, University of Torino, Italy

${ }^{2}$ Department of Psychology, University of Torino, Italy

In the current society, with respect to the past, children have lesser active lifestyles. It is known that sedentary lifestyles contributed to increase the proportion of childhood obesity (Veiga, 2009). This reduce the possibility of developing an adequate level of motor skills (Pühse, 2005), including the coordinative motor skills. In particular, balance is one of the most important coordinative motor skill and it is essential for the control and execution of each movement (Deforche, 2003; Weineck, 2009). The present study aims to investigate the relationship between the dynamic balance, BMI and age.

Dynamic balance was measured by the Eight Balance Test (EBT), in term of number of falls from a modified beam walking. Participant were 164 children attending the second $(47 \%)$ and third class $(53 \%)$ of two primary schools of Turin $(49 \%$ males, mean age $=7.9$ years, $\mathrm{SD}=0.6)$. The main results (obtained by linear regression) showed a positive relationship between BMI and the total falls made on EBT. That is to say, that children with higher values of BMI have a lower dynamic balance, expressed in terms of a greater number of falls on EBT. We also found a negative relationship between age and the total falls made on EBT. This means that older children have more stability while walking than younger children. These results invite reflection on the promotion of physical activity programs targeted on primary school children, enabling them to develop their coordinative motor skills and reduce the spread of a relatively inactive lifestyle and obesity.

Differences in physical activity level in italian high-school adolescents according to sex and age

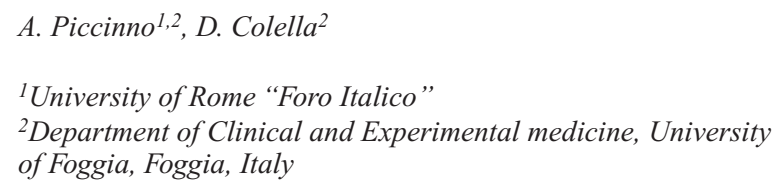

Introduction: Physical activity is an important life-style factor for health promotion. A major health threat for young people in the 21 st century is physical inactivity in conjunction with the elevated prevalence of sedentary behaviour, overweight and obesity $(1,2)$. Despite this evidence, a considerable proportion of children and youth exhibit low levels of activity $(3,4)$. The aim of this study want to examine the differences in physical activity level in Italian high-school adolescents according to sex and age.

Methods: The sample ( $\mathrm{n}=194)$ was composed by males and females attending the 2nd and 3rd class of secondary school located in Foggia and was divided by sex and age groups: 15 years old group (boys: $n=45$, $171,5 \pm 6,3 \mathrm{~cm}, 63,3 \pm 10,3 \mathrm{Kg}$; girls: $\mathrm{n}=36,159,0 \pm 5,0 \mathrm{~cm}, 58,8 \pm 9,1 \mathrm{Kg}$ ), 16 years old group (boys: $\mathrm{n}=57,172,7 \pm 4,9 \mathrm{~cm}, 69,3 \pm 12,5 \mathrm{Kg}$; girls: $\mathrm{n}=31,159,5 \pm 7,0 \mathrm{~cm}, 59,3 \pm 10,4 \mathrm{~kg}$ ), 17 years old group (boys: $\mathrm{n}=15$, $172,4 \pm 4,0 \mathrm{~cm}, 71,9 \pm 10,9 \mathrm{Kg}$; girls: $\mathrm{n}=10,160,2 \pm 5,9 \mathrm{~cm}, 57,1 \pm 11,6$ ). Physical activity level was evaluated by self-report questionnaire IPAQA (5).

Anova One-way was performed to examine the effect of age and sex on walking, moderate and vigorous activity ( $\mathrm{min} /$ week) within each age group. Data were analyzed by SPSS ver.13 and significance was set at $\mathrm{p}<0.05$.

Results:Between 15-16, 16-17 and 15-17 years old groups, significant differences were found in favour of boys in vigorous physical activity $(15-16$ and 16-17: $\mathrm{p}<0.0005 ; 15-17, \mathrm{p}<0.01)$.

In males, between 15-17 years old group, significant differences were found in walking activity with 17 years old group showed better performance than 15 years old group $(\mathrm{p}<0.01)$.

In females, no significant differences were found in all ages in walking, moderate and vigorous physical activity.

Discussion: Physical activity is of crucial importance for physical, motor, mental and social development (6). Prospective study have demonstrated that moderate to vigorous physical activity seems to be associated with health benefits (7). According to several research, this study showed that boys was more active than females in vigorous physical activity and underlined no differences in moderate and vigorous physical activity in all ages.

It's necessary to pay greater attention to this social group: to plan health programmes and to improve moderate and vigorous physical activity in adolescents using correct methodologies.

References

1. Blair S.N (2209) Br J Sports Med, 43, 1-2

2. WHO, 2004

3. Michalopoulou et al. (2011) JSSM, 10, 215-221

4. Spence et al. (2010) JPAH, 7,176-183

5. Hagströmer et al. (2008) Int. J. Obesity, 32, S42-S48

6. Bergier et al. (2012) Ann. Agr. Environ. Med. 109-115

7. Barengo et al. (2007) Hypertens. Rev, 3, 255-263

\section{Effects of an intensive week training program on elderly people}

\author{
E. Padua ${ }^{1-2-5}$, M. Panzarino ${ }^{2-3}$, P. Lebone $^{2-3}$, R. Manno $^{2-6}$, \\ S. D'Ottavio ${ }^{2}$, G. Melchiorri ${ }^{2-4}$, M. Lombardo ${ }^{1-2}$, A. $^{\text {Bellia }}{ }^{1-2}$, \\ B. Ruscello ${ }^{2}$, V. Tancredi ${ }^{2}$, G. Annino ${ }^{2}$
}

${ }^{I}$ Facoltà di Scienze Motorie, Università Telematica San Raffaele, , Roma, Italia

${ }^{2}$ Corso di laurea in Scienze Motorie, Università degli studi di Roma

"Tor Vergata", Italia

${ }^{3}$ Accademia Nazionale di Cultura Sportiva, Roma, Italia

${ }^{4}$ Fondazione Don Carlo Gnocchi IRCCS, Roma, Italia

${ }^{5}$ Scuola dello Sport, Coni-Sicilia, Italia

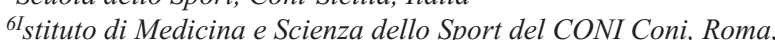

Italia 
To gain benefits from physical performance on older people it is important to establish an optimal training program in terms of volume, intensity, and weekly frequency. WHO recommends aerobic exercise at least 30 minutes on possibly most days of the week and strength training for 2-3 days a week (1). Actually the training programs, commonly used, include a frequency of 2-3 days a week with a duration of 50 minutes each: performing aerobic, strength, balance and flexibility exercise. The aim of this study is to investigate the effects on balance, muscular strength and the flexibility of two different physical activity programs performed within one week. Forty-nine healthy and trained elderly persons, (14 males and 35 females), participating in this study (71,9 $\pm 5,9$ age, 156,8 $\pm 8,3$ height, 74,1 $\pm 11,4$ body weight and 30,2 $\pm 4,38 \mathrm{BMI}$ ), were divided randomly into two groups. The first group (Experimental Group, EG) followed an intensive daily program for one week - twice a day for 45 minutes each session, maintaining the same intensity level of the other group. The training standard session of this group included balance, walking and strength exercises. Moreover, the people of this group performed also two team activity (circuit training with errors and ball games). The second group (Control Group, CG) performed the typical training program, characterized by a frequency of 2 days a week with a duration of 50 minutes each: performing aerobic, strength, balance and flexibility exercise. The subjects of the Experimental Group did not report any physical discomfort neither during nor after the training program. Both groups were tested before and after the training period by investigating their flexibility with the finger to tip floor test (FTTF test), the leg extensors muscles strength with the stand chair test, and balance with one leg stand test. An analysis of variance (ANOVA) for repeated measures with the Newman-Keuls test was used to compare the data between different measurements. The results of the EG showed an improvement in the flexibility of $12,9 \%(\mathrm{p}<0,005)$, in the balance of $38,7 \%$ $(p<0,005)$ and in the leg extensors muscles strength of $24,7 \%(p<0,001)$, whereas the CG showed no significant differences. The results of this study, according to the guidelines of WHO, demonstrate that daily and weekly physical activities (EG) have a beneficial effect on elderly persons. The improved physical performance within a short period of time shows more benefit than the standard physical activities, without comprising the health status (2).

\section{References}

1. WHO, Keep Fit for Life: Meeting the Nutritional Needs of Older Persons, World Health Organization, Geneva, Switzerland, 2002

2: Hrobonova E, Breeze E, Fletcher AE. (2011) Higher Levels and Intensity of Physical Activity Are Associated with Reduced Mortality among Community Dwelling Older People. J Aging Res. Mar $7 ; 2011: 651931$

\section{Hand grip strength in elderly living in residential care facilities: the effects of exercice}

D. Magistro, M. Davanzo, F. Novero, M. Roppolo, A. Mulasso, M. Liubicich

Motor Science Research Centre, Research Unit Motor Skills and Development, SUISM, University of Torino, Italy

With increasing age is usually observed a decrease in motor skills (Stelmanch and Ketcham, 2001). This decrease is often associated with psychological changes (Shumway-Cook and Woollacott, 2000), characterized by a individual variability in the management of activities of daily living (Spirduso, Francis and MacRae, 2005). The reduction of muscular strength which is often associated with ageing may be one of the links between reduced physical performance and functional decline. In fact, the performance of strength of hand function maybe used to identify old people with functional limitations, grade disability and estimate the risk of disability in non disabled people (Giampaoli et al, 1999).
Some older people enter a condition of frailty and lose their independence and living in residential care facilities. The benefits of a regular physical activity for the psychological and physical condition of older people have been already widely acknowledged (ACSM, 2009). In accordance with the guidelines of American College of Sports Medicine (ACSM, 2000) the program of physical activity addressed to older people in the residential care facilities was aimed at preserving the abilities of daily living, which are necessary for conserving a certain independence as long as possible. The present study aimed at investigating the changes between pre-test and post-test after a specific program of physical activity (two session, each of 1 hour per week for 12 weeks) on functionality of upper limbs and hand grip strength, in a sample of institutionalized elderly people.

The sample was composed by 57 older people, 12 men $(21 \%)$ and 45 women (79\%), living in a residential care facilities in northern Italy. The median age is 84 years $(\mathrm{SD}=6,3)$. At the baseline the experimental group and the control group were homogeneous for investigated variables. All the participants live in the residential care facilities permanently and they were classified as partially or totally self-sufficient. We collected baseline and post test measurements for hand grip strength, by a dynamometer. The data were analyzed with T-test for independent samples and repeated measures ANOVA.

The result show that the grip strength of both hands increases between the baseline and post test in the experimental group compared to control group (right hand, $\mathrm{p}=.001$ and left hand $\mathrm{p}=.002$ ).

These results underlined the benefits of the participation at training of physical activity for institutionalized older people. Our findings suggest that the participation in such programmes may be especially important for the maintenance of specific components of physical functioning. However, these results showed that also in critical condition, as that of the older people living in residential care facility, the introduction of a relatively simple training may have positive effects on the individual functioning in short time.

\section{Analysis of heart rate variability in heart rate recovery to exercise in young athletes}

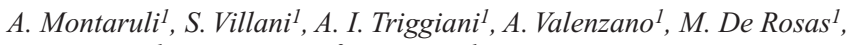
M. I. Roselli' ${ }^{1}$, L. Capranica ${ }^{2}$, G. Cibelli ${ }^{1}$

${ }^{1}$ Dept. Clinical and Experimental Medicine, University of Foggia, Foggia, Italy

${ }^{2}$ Department of Human Movement and Sport Sciences, University Foro Italico, Rome, Italy

Objective: The aim of this study was to examine the possible association between Heart Rate Variability (HRV) and the Immediate Recovery Index (IRI), an arbitrary score of physical fitness, based on evaluation of post-exercise Heart Rate Recovery (HRR).

Methods: Twenty male athletes $(23.2 \pm 4.2$ years $)$ partecipated in this study. Subjects underwent a standardized 3-min step test and IRI was determined. HRV was measured in a 5-min segments before (pre-) and after (post-) exercise, with subjects in supine position. Mean RR interval, standard deviation of RR intervals (STDRR), root mean square of differences (RMSSD) of successive RR intervals, low-frequency power (LF, 0.04-0.15 Hz), high frequency power (HF, 0.16-0.40 Hz), instantaneous beat-to-beat (SD1) and continuous beat-to-beat (SD2) variability were computed. Heart rate (HR) was recorded at the end of the test, (expressed as HRpeak and as percentage of the individual HRmax), within 60 (R1) and 90 (R2) sec post-exercise. IRI was calculated through the following equation: [100 x sec of exercise duration]/[5.5 x (HR1-HR2)]. Exercise intensity levels were determined by the Rating of Perceived Exertion (RPE) scale. Data were presented as $\mathrm{M} \pm \mathrm{SD}$. The Student's t test for paired 
samples was used to analyze the effect of the test upon variables. The Pearson product-moment correlation coefficient was used to study the associations between variables. Statistical significance was set at $\mathrm{P}<0.05$. Results: At the end of the test, mean HRpeak value was 191.7 beats min1, corresponding to $97.5 \%$ of individual HRmax,. Mean RPE was 3.7. Mean IRI was 62.0 (range 43.7-90.9), corresponding to an acceptable level of post-exercise HRR. Compared to pre-exercise, post-exercise lower mean RR (847.7 to $678.7 \mathrm{~ms}$ ), STDRR (53.4 to $31.1 \mathrm{~ms}$ ), RMSSD (36.9 to 13.1$)$, LF (1067.6 to $311.7 \mathrm{~ms} 2), \mathrm{HF}$ (628.7 to $84.0 \mathrm{~ms} 2$ ), SD1 (26.1 to $9.2 \mathrm{~ms}$ ) and SD2 (70.4 to $42.6 \mathrm{~ms}$ ) values were observed. The Pearson product-moment correlation coefficient showed significant associations between IRI and pre-exercise mean RR intervals $(r=0.61)$, STDRR $(r=0.47)$ and SD2 $(r=0.51)$. IRI significantly correlated to postexercise mean RR intervals ( $\mathrm{r}=0.87)$, STDRR $(\mathrm{r}=0.63)$, RMSSD $(\mathrm{r}=0.59)$, power LF $(\mathrm{r}=0.50)$, SD1 $(\mathrm{r}=0.59)$ and SD2 $(\mathrm{r}=0.62)$.

Conclusions: The major finding of this study was that, due to its intrinsic non-stationary character, the autonomic imbalance underlying postexercise HRR, monitored by IRI, was better evaluated through non linear HRV analysis as than conventional HRV parameters.

\section{The acute effects of isocapnic hyperpnea on spinal column flexibility}

\section{Gollin, L. Beratto, C. Scagliotti, M. Roppolo}

Motor Science Research Center, School of Exercise \& Sport Sciences, University of Turin, Italy

Aim: The study evaluated the variation in the flexibility of the spinal column after an acute respiratory training session based on isocapnic hyperpnea in a group of professional women soccer players.

Methods: Ten professional women soccer players took part in this study (age: $21 \pm 4$ years (mean \pm SD), height: $166 \pm 5 \mathrm{~cm}$, weight: $62 \pm 6 \mathrm{~kg}$, BMI: $22 \pm 2 \mathrm{~kg}$, and with $12 \pm 5$ years of training). The following devices were used:

- The Spirotiger ${ }^{\circledR}(\mathrm{ST})$, for respiratory training in isocapnic hyperpnea (MVM, Italy), which measures the total volume of air breathed by the athlete;

- The SpinalMouse ${ }^{\circledR}$ (SM), for morphological evaluation of the spinal column and of its joint mobility, which provides a number of parameters as listed in the following: 1) Chest segment (ThSp): the angle between the first ThSp1 and the last thoracic vertebrae ThSp12;2) Lombar segment (Lsp): the angle between the last thoracic ThSp12 and the first sacral vertebrae S1;3) Sacral angle (Sac/Hip): the angle created by the contour of the sacral segment with respect to the plumb line; 4) Inclination (Inc): the connection between ThSP1 and S1 defines the inclination line. The angle created between this line and the plumb line is the Inc angle. 5) Length of segment ThSp1 - S1 (Length): the length of the back measured from $\mathrm{C} 7$ to $\mathrm{S} 1$ expressed in millimetres (value corresponding to the trajectory of the instrument).

To avoid biased results between the conditions with (W) and without (WO) ST the counter balance design was used.

Results: Statistically significant differences (Wilcoxon test, WO vs W) were observed both in the ThSp $(\mathrm{P}<0,05,-16 \%)$ and in the length of the extended spinal column ( $\mathrm{P}<0,05,-3 \%)$, which were found to be lower after the acute respiratory training session.

Discussion/Conclusion: The acute application of respiratory training shows a reduction in spinal column flexibility, highlighting how training is a process to be carried out chronically.
Handgrip strength and 1RM bench press performance: a novel approach to evaluate upper body maximal strength

\author{
D. Filingeri ${ }^{1}$, E. Thomas ${ }^{2}$, M. Raccuglia ${ }^{2}$, A. Paoli ${ }^{3}$, A. Bianco $^{2,4}$, \\ A. Palma ${ }^{2,4}$ \\ ${ }^{1}$ Environmental Ergonomics Research Centre, Loughborough \\ University, $U K$ \\ ${ }^{2}$ University of Palermo, Department of Sport and Exercise Sciences \\ (DISMOT), Italy \\ ${ }^{3}$ University of Padua, Department of Biomedical Science, Italy \\ ${ }^{4}$ Scuola dello Sport, CONI Sicilia, Italy
}

In the last few years, handgrip strength has been widely recognized as a relevant indicator of physical function, nutritional status and quality of life in a clinical population. However, the scientific literature is still lacking of knowledge regarding the use of this indicator within a general healthy and physically active population. The possibility to use the handgrip strength as an indicative value of some specific physical qualities, such as the upper body maximal strength (expressed in terms of $1 \mathrm{RM}$ bench press), can be considered an unexplored field and its development might represent a relevant support for the strength and conditioning professionals. Therefore, we hypothesize that a significant association might be found between handgrip strength, body composition and upper body maximal strength and that this association might be used as a specific "performance predictor". Fifteen healthy subjects ( 8 men $/ 7$ women; age $25.53 \pm 7.14 \mathrm{yr}$;! body mass $65.58 \pm 13.26 \mathrm{~kg}$; height $167 \pm 10 \mathrm{~cm}$ ) without habitual intensive exercise participated in this pilot intervention. Body composition, handgrip strength and maximal body upper strength were evaluated. Subjects' body composition was estimated through a bioelectrical impedance analysis and resulted in a mean value of $22.12 \pm 8.64 \%$ of fat and $77.81 \pm 8.5 \% 1$ of lean mass. Handgrip (HG) strength was estimated for both dominant and not dominant side, respectively $(39.27 \pm 14.52 \mathrm{w} ; 37.59 \pm 13.49 \mathrm{w})$ through the use of a handgrip dynamometer. Subjects' upper body strength was tested using a bench press 1RM test with an already standardized protocol. Subjects' maximal strength resulted in an average of $49.21 \pm 23.87 \mathrm{~kg}$. HG and $1 \mathrm{RM}$ testing were overseen by the same investigator and conducted with the same equipment. Each subject was instructed to refrain from any strenuous activities for 72 hours before testing d! ay. STATISTICA Software package $\subset$ for Windows $\subset$ was adopted wh en appropriate. Overall the findings showed a strong relation (Pearson's $r=0.77$ ) between HG and 1RM bench press in both, male (0.41) and female $(0.80)$ but a low association with Free Fat Mass. Unexpectedly, female have higher association than males. These preliminary results shown that the handgrip strength might be associated to the individual maximal strength performance and it might be considered as a valid predictor of upper body strength performance within a sport/fitness context, though the presented pilot intervention we cannot draw any conclusion on the topic, due to the limited sample analyzed.

\section{Synaptic plasticity in hippocampal mice slices after exercise $t$ raining}

\section{G. D'Arcangelol, E. Padua ${ }^{2}$, T. Triossi ${ }^{1}$, L. Dal Tò̀ ${ }^{1}$, G. Melchiorri ${ }^{1}$, C. Frank ${ }^{3}$, V. Tancredi ${ }^{l}$}

${ }^{1}$ Dip. Medicina dei Sistemi, Univ. Tor Vergata, Roma, Italy ${ }^{2}$ Univ. San Raffaele, Roma, Italy

${ }^{3}$ Centro Nazionale per le Malattie Rare, ISS, Roma, Italy 
It is well known that exercise training (ET) plays a protective role in aging and neurodegenerative disorders. Hippocampal CA1 pyramidal cells are referred to as "place cells" because generate an internal representation of the external environment. In order to analyze the long-term effects of physical training on synaptic plasticity in the pyramidal layer of hippocampal CA1 region, a structure involved also in spatial learning, $\mathrm{BALB} / \mathrm{c}$ mice 40 days old were divided in four groups: the first one was used as sedentary control (SC), while the other groups were trained for 6 weeks, three times a week, by using the rotarod performance system. We employed three different protocols, consisting in incremental volume training (50 and $100 \%$ of volume) and in intermittent training. Muscular strength before and after the exercise training was also evaluated. The trained animals exhibited both a prolonged endurance ( 6 minutes versus 30 minutes) and an increased strength $(30 \%)$ in all the three trials tested respect to the sedentary controls. The strength was evaluated by hanging the animals to a force transducer. After the exercise training period the animals were sacrified and the hippocampal slices were prepared according to the conventional methods. High frequency stimulation induced long term potentiation (LTP) in slices obtained from sedentary mice, while trained animals exhibited different trends: in incremental trained ones the induction phase was affected while the maintaining phase was similar to the control slices. In the intermittence training group instead the LTP was totally blocked. These results indicate that long term exercise training acts in increasing strength and muscle endurance, but also suggest a modulatory role in synaptic plasticity that has to be deeply investigated.

\section{Does a specific genetic background influence endurance or power-orientated phenotypes?}

\section{P. Proia ${ }^{1,3}$, V. Contrò ${ }^{\text {, }}$ A. Bianco ${ }^{\text {I, G. Schiera }}{ }^{2}$, P. Saladino ${ }^{2}$, M. Traina ${ }^{1}$, A. Palma ${ }^{1}$}

${ }^{1}$ Dipartimento di Studi Giuridici, Economici, Biomedici, Psicosociopedagogici delle Scienze Motorie e Sportive, DISMOT, Università degli Studi di Palermo, Italy

${ }^{2}$ Dipartimento di Scienze e Tecnologie Molecolari e Biomolecolari, Università degli Studi di Palermo, Italia

${ }^{3}$ Department of Oncology and Diagnostic Sciences, University of Maryland, Baltimore, MD, USA

The purpose of this study was to determine the probability of individuals having the "best" mitochondrial biogenesis related polygenic profile that could increase performance.

We compared polygenic profile analyzing several polymorphisms on sixty professional italian soccer players, considered "power-oriented athletes" and thirty sedentary volunteers. Samples of venous blood were obtained by standard clinical procedures and anticoagulant-treated blood was used to prepare genomic DNA. The polymorphic sites were scanned using PCR-RFLP protocols with different enzyme. Furthermore, a cloted part of venus blood sample was used to obtain serum from which we analyzed total cholesterol, high density lipoprotein cholesterol, low density lipoprotein cholesterol and triglycerides, by using a kit in a chinetic enzyme analyzer.

We computed an "endurance genotype score" (EGS), related to mitochondrial biogenesis, from 0 to 100 , from the accumulated combination of the polymorphisms in the PPAR $\alpha$-PPARGC1A-NRF2 (A/G; C/T) pathway; moreover, we analyzed the ACTN3 polymorphism. Particularly, as regards PPAR $\alpha$ we also analyzed the lipid profile (total cholesterol, triglycerides, HDL and LDL) because it is an important factor that regulate the balance between fatty acid and glucose metabolism.

The results evidence an higher EGS and a variation of genotype distribution of the analyzed polymorphisms in professional soccer players compared with sedentary healty volunteers. Moreover, our study shows that $\operatorname{PPAR} \alpha$ genotype distribution is not related with a variation in the values of the lipid profile.

In conclusion professional soccer players possess "theoretically" a genetic background that is more suitable for mitochondrial biogenesis.

\section{Kinetics of cardiovascular responses to apnea during light-intensity exercise}

\section{A. Sivieri ${ }^{I}$, M. Capogrosso ${ }^{I}$, R. Perini ${ }^{1}$, G. Ferretti ${ }^{1}$ \\ ${ }^{1}$ Dept. Biomedical and Biotechnology Sciences, University. of Brescia, Italy}

The cardiovascular responses to breath-holding are such that, after an initial rapid decrease in heart rate (HR) and increase in blood pressure (BP) (phase I), these parameters remain stable for about $2 \mathrm{~min}$ (phase II). Then (phase III) i) HR further decreases; ii) BP continuously increases; iii) intrinsic variability of all parameters progressively becomes larger. Our hypothesis is that the start of phase III corresponds to the physiological breakpoint of apnea, occurring when arterial gas composition is such as to stimulate ventilation and involuntary diaphragmatic contractions. If this is so, phase II duration must be reduced during exercise, when metabolic rate (M') is augmented. To test this hypothesis, 8 male divers ( $36 \pm 7$ years) performed voluntary maximal apnea: A) sitting at rest and B) pedaling on a cycle ergometer at $30 \mathrm{~W}$. Before, during and after apnea, arterial pressure profiles were continuously recorded by means of a noninvasive cuff pressure recorder, together with arterial oxygen saturation ( $\mathrm{SaO} 2$, infrared spectrometry). Alveolar gas exchanges were determined before and after breath-holding by a metabolic card. In A, apnea lasted $227 \pm 62 \mathrm{~s}$. Three phases were recognized. In phase I ( $29 \pm 3 \mathrm{~s})$, HR decreased from $98 \pm 11 \mathrm{~b} / \mathrm{min}$ to $82 \pm 13 \mathrm{~b} / \mathrm{min}$, systolic BP (Ps) remained unchanged $(140 \pm 11 \mathrm{mmHg})$, diastolic $\mathrm{BP}(\mathrm{Pd})$ increased from $73 \pm 12$ to $82 \pm 10 \mathrm{mmHg}$. In phase II $(102 \pm 32 \mathrm{~s})$, all parameters remained stable. In phase III, HR fell to $57 \pm 5 \mathrm{~b} / \mathrm{min}$, while Ps and Pd increased, respectively, to $215 \pm 23 \mathrm{mmHg}$ and $105 \pm 16 \mathrm{mmHg}$ after $110 \pm 35 \mathrm{~s}$. SaO2 was $91 \pm 5$ $\%$ at the end of phase II and $75 \pm 13 \%$ at the end of apnea. In B, M' was twice as at rest. Apnea lasted $88 \pm 21 \mathrm{~s}$. Cardiovascular time courses showed two phases. During the first ( $24 \pm 7 \mathrm{~s}$, phase a), cardiovascular parameters remained unchanged. HR ( $112 \pm 9 \mathrm{~b} / \mathrm{min})$ was $15 \&$ plusm $\mathrm{n} ; 10$ $\mathrm{b} / \mathrm{min}$ above exercise steady state values; Ps was $167 \pm 15 \mathrm{~mm} \mathrm{Hg}$ and Pd $81 \pm 15 \mathrm{mmHg}$. In the following $64 \pm 22 \mathrm{~s}$ (phase b), a continuous fall of HR and increase in Ps and Pd occurred. At the end of apnea, HR was $55 \pm 10 \mathrm{~b} / \mathrm{min}, \mathrm{Ps} 244 \pm 24 \mathrm{mmHg}$ and $\mathrm{Pd} 112 \pm 13 \mathrm{mmHg}$. SaO2 started to drop after $\sim 30$ s of apnea and was $76 \pm 11 \%$ at the end. In conclusion, HR and BP time courses differed in the two conditions. Instability of cardiovascular values after phase a and the early, steep decrease in $\mathrm{SaO} 2$ suggest that during exercise apneas chemoreflex was activated in advance compared to resting apneas. The consequence was the progressive bradycardia and hypertension observed in phase $b$, to attain cardiovascular and $\mathrm{SaO} 2$ values similar to those of end of apnea phase III at rest. The absence of initial HR drop in apnea in exercise indicates that the level of autonomic activity affects the first cardiac response to breath-holding. 


\section{ORAL PRESENTATIONS}

\section{Saturday, October $6^{\text {th }}, 2012$}

\section{Session 5.1. Motor Learning and Development}

\begin{abstract}
The relationship between gross motor skills and math achievement during childhood: mediation effect of ADHD symptoms
\end{abstract}

G. Bardaglio I, D. Marasso ${ }^{1}$, F. Magno ${ }^{1}$, E. Rabaglietti ${ }^{1,2}$, G. Zucchetti ${ }^{2}$, S. Ciairano ${ }^{1,2}$

${ }^{I}$ Motor Science Research Centre, Research Unit Motor Skills and Development, SUISM, University of Torino, Italy

${ }^{2}$ Department of Psychology, University of Torino, Italy

Aim: The relationship between motor skills and academic achievemen has been especially investigated in children with atypical development (Viholainen et al., 2006). Research has shown that well-developed gross motor skills facilitate the development of children's academic abilities in general and their abilities in reading and math in particular (Son \& Meisels, 2006). However, it is important to also investigate this association outside the field of disability, as the present study aims, in order to verify whether motor skills have an equally important role on academic achievement, especially in math, and whether the presence of behavioral problems, such as symptoms of Attention Deficit Hyperactivity Disorder (ADHD), can mediate this relationship in children.

Methods: Sixty-three children who attended the third grade of primary schools in Piedmont, a region of northwest Italy, participated in the study $(52 \%$ boys, mean age $=8.4$ years, $\mathrm{SD}=0.4)$. The children's gross motor skills were measured by the Test of Gross Motor Development (Ulrich, 1985). Teachers completed a self-report questionnaire about the children's academic achievements and symptoms of attention deficit hyperactivity disorder (the ADHD Rating Scale for Teachers; Cornoldi et al., 1996). The linear regression method as well as Sobel's test were used for the analysis.

Results: The results show that gross motor skills have positive effects on any academic achievements, especially on math, due to the mediation of ADHD (Sobel's Z-value $=2.32 ; \mathrm{p}=.02$ ).

Conclusions: This findings suggest to individuate efficient intervention programs in order to promote motor skills and in this way contributing to improve school achievement in all children.

Conductive pedagogy as educational action for young people and adults with motor disability
D. Costantino ${ }^{1,2}$
${ }^{1}$ University of Palermo, Italy
${ }^{2}$ School of Sports Science CONI Sicily, Italy

Introduction: Conductive pedagogy is a particular application of education science. It is founded on the ideas, educational approaches and systems of views of András Petö. He set a perfect example of the conductive education system, thanks to a new holistic approach at life (a new Weltanschauung). This perspective built the principles of completeness and understood the intensive integrity of human existence. New holistic approach means that world and man are viewed in a complex fashion. Education has an impact on the entire person, concretely on the whole personality. Man, in his essence, is multifactorial and has a many-sided personality. If conductive pedagogy want to be a successful and effective treatment, it must be many-sided and multifactorial. Conductive pedagogy has an indisputably 'holistic' approach because the human body can be influenced and developed through the soul. The conductive pedagogy is a scientific item of study by Franz Schaffhauser of University of Budapest (my guide in this new field of studies).

Methods: The central endeavour in the implementation of the Peto method in the conductive education process is to conduct human being as a concrete person to realising their own intensive wholeness, to assist and support them in order to promote their own values and dignity. During the process the educator's interest is directed towards the fulfilment of the particular completeness and meaning of human life. Conductive education can be interpreted as a positive orientation towards experiencing entirety, towards a meaningful life, towards meaningfulness. Conductive education means shaping appropriate reference to meaning.

Results: Conductive pedagogy needs a realistic concept of man and organisation of educational procedures.

In summary, we can affirm that conductive pedagogy as an applied human discipline has its own philosophical-anthropological region of sources which is kept in evidence and serves as a basis of verification and legitimation (and a background of integration). Thanks to conductive pedagogy, people (young and adults with motor disability) can have the chance to look at themselves like a "wholeness" and like "persons", not giving in to despair (or to a temptation) to give out and surrender to their handicaps.

Discussion: Education has an impact on the entire person. Conductive pedagogy needs to be many-sided, if it want to be useful to persons. «Spiritual dimension is human dimension which penetrates the physical and spiritual dimension and makes man, every human being, complete and a person. Being a human means being a complete whole. This completeness, whole-ness is the foundation of the human person's, value and dignity» (F. Schaffhauser, 2012).

References

- Frankl, V. (1959/2006) Man's search for meaning, Beacon Press, Boston (5th Ed.)

- Moreno, J. L. (1953) Who shall survive?, Beacon House Inc., Beacon N.Y (2nd Ed.)

- Hari Maria (1997) A konduktiv pedagogia tortenete, MPANNI, Budapest

\section{Study of gross motor skill performance in kindergarten children}

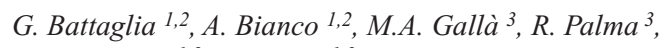
M. Bellafiore ${ }^{1,2}$, A. Palma ${ }^{1,2}$

${ }^{1}$ Department of Sports Science (DISMOT), University of Palermo, Italy

${ }^{2}$ Regional Sports School of CONI Sicilia, Italy

${ }^{3}$ Faculty of Sports Science, University of Palermo, Italy

Several studies show an high decline in coordinative motor skills in childhood (Roth et al., 2009). For this reason, promotion of physical activity and motor skills appear to be a valuable mean to improve children's development in today's kindergartens. The purpose of the investigation was to study the effects of a specific training program to train gross motor skills in kindergarten children. Forty-one preschool children (27 females and 14 males) were cluster randomized into either a control group [CG] (n: 19; age: $4.68+/-0.82$ years; height: $1.11+/-0.07 \mathrm{~m}$; weight: $21.51+/-4.88 \mathrm{~kg}$ ) and a trained group [TG] (n: 22 ; age: $4.55+/-$ 0.51 years; height: $1.12+/-0.06 \mathrm{~m}$; weight: $21.95+/-4.87 \mathrm{~kg}$ ). The Test of Gross Motor Development was used to test loco-motor abilities and object- control skills. The test evaluates 12 abilities of which seven locomotor skills (run, gallop, jump on the same foot, jump forward, long 
jump, little jumps forward and laterally) and five object-control skills (catch a ball with a tennis racket, bounce of the ball from stationary posture, catch the ball with two-hand, kick the ball running, throw a ball with a hand). Raw, standard and Gross Motor Development Quotient (GMDQ) were calculated for each participant. TG was trained for 4 weeks by two sessions/week. In particular, every trained session included: a warm up period ( $\sim 10 \mathrm{~min})$, a training period ( $\sim 40 \mathrm{~min})$ including exercises to train locomotor and object-control skills, cool down period ( $\sim 10 \mathrm{~min}$ ). CG did not perform any sport activity during the experimentation. All data were acquired before and after the experimentation. Data showed that TG improved raw, standard scores and GMDQ than CG after the training period. In particular we found a $90 \%$ and $137 \%$ improvement in locomotor skills and object-control skills respectively after 4 weeks of exercise training. So, our training program appear to be able to improve gross motor skill performance in preschool children in agreement with Matvienko's study (20 10) that showed as an intense and short school training program can generate sustainable improvements in motor skill and fitness levels in kindergarten and first-grade students.

References

- Matvienko O, Ahrabi-Fard I (2010) The effects of a 4-week afterschool program on motor skills and fitness of kindergarten and firstgrade students. Am J Health Promot. 24(5):299-303.

- Roth K, Ruf K, Obinger M, Mauer S, Ahnert J, Schneider W, Graf C, Hebestreit H (2010) Is there a secular decline in motor skills in preschool children? Scand J Med Sci Sports.;20(4):670-8.

\section{Peer education and management of sport teams: pedagogical proposal and educational praxis}

\section{Costantino ${ }^{I, 2}$ \\ ${ }^{1}$ University of Palermo, Italy \\ ${ }^{2}$ School of Sports Science, Italy}

Introduction: Peer Education is educational and teaching strategy. It aims at complete (human and character-forming) growth of person connected with "peer group» (i.e. people who share same world of values and Weltanschauungen). Peer Education can become real "pedagogical tool" to improve "education at health» and coordination/promotion of pupils and athletes. Thanks to Peer Education, it is more simple to set in motion natural exchange among "people of peer-condition and of peerstatus". They will put in common knowledges, emotions, experiences, creating tight ties leading to harmony and general agreement.

Methods: Thanks to conscious action of physical trainer, Peer Education helps young in knowing themselves, increasing their faculties of feeling in a critic way and developing self-consciousness. Individual (pupil or athlete) is a member of the group and, at the same time, necessary mean to permit the growth of peer-group. He hands down (from himself to peer-group) his world of emotions and feelings, aiding to socialize each other all member of the group. A group that - as time goes by - becomes "real team of peer".

Thanks to Peer Education, pupils (or athletes) become "main characters" of their pedagogical/educational iter, of their paidéia. Sure, at first step, physical trainer plays a fundamental role, but, little by little, pupil/athlete gains life skills and so he is able to enter in touch with all world round him. Educational praxis follows - without straining - curricular program.

Results: It is confirmed that Peer Education spurs pupils (and athletes) to look for more health-conscious ways of life. Richer ways of life that let young improve management of their bodies and self-consciousness. For young it is stimulating to make comparison with peer-group. After knowing the others, they are more ready to accept the differences that are in our world. There is no more fear to make wrong. It remains a will to learn, creating common itinera of growth.

Peer Education brings educational roles into play, and it is a keyword for creating new cultural forma mentis.

Discussion: «Education at movement and sport» plays two different roles, developing bodies and minds of pupils and athletes. Peer-group builds "a common world", comparing competences, knowledges, actions and tasks (in a new perspective of accountability). It will be the new classroom of peer-group that will build educational program and organize sports activities.

References

- Collier G. (1983), The Management of Peer-group learning: syndicate methods in higher education, Guilford, Society for research into higher education at the University of Surrey

- Falchikov N. (2001), Learning together: peer tutoring in higher education, Routledge, London-New York

- Pellai A., Rinaldin V., Tamburini B. (2002), Educazione tra pari: manuale teorico-pratico di empowered peer education, Trento, Erickson

Evolving role of physical education on psycho-motor development in children with disabilities

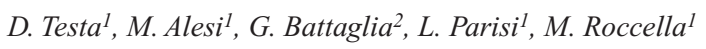

${ }^{I}$ Department of Psychology, University of Palermo, Italy ${ }^{2}$ DISMOT, University of Palermo, Italy

An educational intervention focused on the lived experience motor can promote the necessary feeling of security and autonomy, and to help the disabled child is in the evolutionary process in the path of integration. The physical education, centered on the size of the body, encourages the encounter of the other through physical contact and mutual perceptions, the routes of communication, relationship and experience, and this highlights the relationship between motor performance and development cognitive, which was and is an important object of study of psychology and pedagogy. Numerous studies have shown that, especially during the developmental age, they might be more precocious maturation of some parameters in subjects practicing intellectual-recreational activities and sports and how the logical operations are anticipated and facilitated from bodily experience. Other studies have shown that physical activities are under the control of thought processes and how various cognitive skills can be acquired and modified through movement. From a psychological standpoint, the sports training is seen as a type of cognitive learning, an area associated with emotional and a social. Processes are involved in learning motor attentional, perceptual, mnemonic and decision making. The exercise enhances the ability to analyze situations, select the relevant indices, organize and create plans for one or a series of movements and use information available retroactively, within the limits of development of each. Therefore in the context of disability, it highlights the importance for the acquisition of motor skills of independence, fundamental to the quality of life of the disabled person. If it gives the movement a mental dimension and interactive we can feel the motor learning as the acquisition of patterns and rules that create and control the action is assumed that cognitive and social character. The motor action seen as the result of a cognitive process appears to be an important tool for achieving autonomy, as well as developing the skills of reflection on experience, that raises from the town, the comparison and the verbalization of the motor done. The purpose of this paper is to identify the goals that physical education can and must stand, providing teachers and especially teachers to support the useful tools to identify the teleological and axiological dimension that must mean the educational act, through the analysis motor learning and its implications in cognitive development and the role of the body and the body in communication. 


\section{Motor control and motor learning paradigms in exergame design}

\section{P.A. Di Tore, G. Raiola}

DISUFF - Dept Human, Phylosophycal and Educational Sciences, University of Salerno, Italy

This paper documents the experimental stages of development and alpha testing of a didactic exergame designed at the University of Salerno. Software development has been inspired by the finding, based on literature review, of the lack of exergames designed specifically to support motor activities teaching. The state of the art survey on exergaming has shown that there are no products based on a solid theoretical framework oriented to motor learning. The motor learning definition which we refer is that proposed by Schmidt and Wrisberg:

"Motor learning is an internal process that reflects the level of individual ability and performance could be evaluated according to the relative stability of the execution of a task." (Schmidt \& Wrisberg, 2008)

The software module developed has as objects of study the reaction times (RT) in a digital environment. An exergame based on Microsoft Kinect has been developed, where the player controls an avatar on the screen. The target audience is the age group of primary school.

The game goal is to strike with the hands the clouds (stimuli) that appear randomly in the playing area and move to the right or left. If the player hits the clouds, he earns a point; otherwise, the clouds disappear from the board. The game ends when the player misses 10 clouds. As the player earns points, clouds speed increases.

Leveraging Kinect's ability to identify the user's body segments, the clouds-stimuli were divided into two categories and associated with the player's hands, so that some stimuli can be hit only with the left hand and others only with right hand. The avatar hands were stained corresponding to the two stimuli to simplify the recognition (red clouds to hit with red hand, green clouds to hit with green hand). The data collection form has been designed to distinguish high compatible stimuli from low compatible stimuli.

"Stimulus-response (S.R) compatibility is usually defined as the naturalness of the connection between the stimulus and the associated response. For example. using the right hand rather than the left to respond to a stimulus to move right is more compatible because both the stimulus and the hand are moving in the same direction (to the right)". (Schmidt \& Wrisberg, 2008)

The research hypothesis is that general laws regarding the relationship between the number of SR pairs and reaction times also apply in the virtual environment and that, therefore, reaction times increase systematically in presence of low compatible stimuli.

The alpha testing was conducted on a sample of 50 primary school students on Procida island, at the national event "W I bambini". The analysis of the collected data confirms the initial hypothesis. The statistical analysis was conducted for now using standard position indexes and we reserve subjecting the collected data to a test of hypothesis.

\section{References}

- Schmidt, R. A., \& Wrisberg, C. A. (2008). Motor learning and performance: a situation-based learning approach: Human Kinetics Publishers.

\section{Intellectual disabilities and physical education}

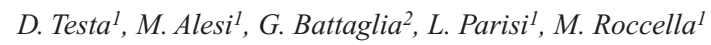

${ }^{1}$ Department of Psychology, University of Palermo, Italy

${ }^{2}$ DISMOT, University of Palermo, Italy

The intellectual disability is often related to difficulties or failure of some forms of learning and development, in which the sensory-perceptual-motor activities play an important role. Physical activity aimed at the sport becomes an important tool in the process of social integration, as it allows the educator to create strong ties between the pupil and the outside world. From a psychological standpoint, the sports training is seen as a type of cognitive learning, an area associated with emotional and a social. If we focus our analysis we see that the appearance of motor learning cognitive psychology of sport has carefully considered as a movement is learned and how this can be integrated with action patterns that create complex motor skill of the athlete. Processes are involved in learning motor attentional, perceptual, mnemonic and decision making. It can be argued that the ability to process information is in operation:

- At the entrance, sensory and perceptual operations;

- The central processes of organization and management of information subsequent to an activity of problem solving and decision making;

- Outgoing, motor function and retroactive use of the information.

The 'exercise enhances the ability to analyze situations, select the relevant indices, organize and create plans for one or a series of movements and use information available retroactively, within the limits of development of each. Another feature of the space motor is to be "symbolic space", and producer of shared meanings. The knowledge of the meanings attributed to the act, or play sports, gives a specific command such as the ability to assume the role of the other. Learning a gesture, the subject acquires also a system of meanings associated with it. Pursuing a motor activity aimed the student with intellectual disabilities can learn to master them or get more roles, to make the cognitive relaxation indispensable for the achievement of a result. In the activity in motor and behavioral and motor sports space is closely correlated with that representative. Each person creates a cognitive map, variable, its action and that of others, in some cases. This process of development of cognitive maps is not given by a factor of purely repetitive, but rather to relate to a reality based on goals and objectives that underlie different strategies and different abilities. According to the type of physical activity the person is having to operate within a system of multiple relationships. The motor action, or play sports, often involves the use of two integrated systems for coding spatial information intake:

1. Self-related, which could translate into self-organization: When the athlete prepares to jump an obstacle using the mechanisms of anticipation, planning, execution and control of the gesture.

2. Landmark-related or spatial organization: gives meaning to the situation where the athlete is immersed and which allows him to choose and arrange the appropriate motor action.

The proper use of the two methods can have a better sense of direction and space. Therefore in the context of disability, it highlights the importance for the acquisition of motor skills of independence, fundamental to the quality of life of the disabled person. The purpose of this paper is to identify through analysis of motor learning, its implications in cognitive development.

\section{Daily physical activity assessment in school children: 'Movimentiamoci Diary' validation study"}

\section{G. Rossi ${ }^{1}$, M. G. Vinciguerra ${ }^{1}$, P. Izzicupo ${ }^{2}$, R. D'Egidio ${ }^{2}$, V. Carnicelli ${ }^{l}$, M. Scatigna ${ }^{I}$}

${ }^{1}$ University of L'Aquila, Italy

${ }^{2}$ University of Chieti-Pescara, Italy

In a behavioural epidemiology perspective there are different dimensions for featuring and assessing physical activity (PA) profile: type, frequency, duration, intensity, correlates, outcomes. Scientific evidences on health and PA led to recommendations for children and adolescents. They should be at least moderately active for at least one hour a day with 
a wide range of possible activities: sports, physical education at school, free range play, active transport, dance, housework.

In 2012 we carried out a validation study to assess criterion validity of an Italian Daily Physical Activity Diary (IDPAD), targeted at primary school children and originally created from overseas examples. 51 pupils (10-11 years old) of Abruzzo region (middle Italy) filled in IDPAD for four consecutive days, registering everything they do from 7.00 am to $12 \mathrm{pm}$ in 68 intervals of 15 minutes. Using a coded system, they specified for each interval the kind of activity (e.g. sleeping, having lunch, getting dress, school lessons, sports, walking, homework, etc) and their perceived intensity (i.e. light, moderate, vigorous, very vigorous activity). We obtained METs-transformed data and the number of errors that occurred when reported intensity was too high or low compared with coded criteria.

In three out of four days, a subsample of 18 children wore in a belt around their waist an electronic device, the Liferecorder - Plus accelerometer, collecting objective measures of PA intensity (METs) and motion (steps).

For overall 54 days coupled data, self-report $v s$ objective measures have been compared by means of pairwise Spearman's Rho.

Correlations between METs values for single intervals have been found not sufficient (rho $\leq 3.0$ ) nor significant. However we found a significant sufficient correlation between the daily amount of bouts classified as LPA intense (Low PA, rho $=0.42, \mathrm{p}<0.01$ ) and MVPA intense (Moderate to Vigorous PA, $0.47, \mathrm{p}<0.001$ ).

MVPA level appears significantly higher in males than in females and it increases from week-days to week-end. At same time, sedentary activity (SA) increases in the week-end, interestingly confirming what recent scientific literature is showing: PA and SA have to be considered separated patterns of behavior, with different mechanisms of influence on health outcomes.

Our study demonstrated that the IDPAD has some limits due to subjective measures so it couldn't be considered an exact instrument for assessing absolute energy expenditure in a quantitative analyses (e.g. causal relationship evaluation in epidemiological studies). However it is valid as raw quantitative assessment of daily child's PA level. Nevertheless it makes subjects aware about what kind of activities they do more or less and how practically change their sedentary lifestyle. So, daily self-reporting PA by means of diary is a good instrument for health promoting interventions, from individual goal setting to impact assessment at school and population level.

\section{Mária Hári and her Conductive Pedagogy}

\section{S. Costantino}

Dip. Scienze pedagogiche e psicologiche “G. Catalfamo”, Messina, Italy

Introduction: The number " 2 " was very important in the life of Mária Hári, the follower and first helper of Dr. András Petö, the Hungarian inventor of Conductive Education in the first half of the past century. Mária was 22 years old when she met Petö and began to know about theories regarding his method of education and rehabilitation of patients with motor disabilities. And Mária was 44 years old when Petö, the fascinating and sometime rude doctor creator of Con-ductive Education, left this world. She continued the oeuvre of Petö with untiring patience and indefatigable work. With her intelligence and high-level skills Mária Hári was the "origi-nator of college-level conductor training" Petö Institut of Conductive Education of Budapest.

Methods: Conductive Education is a complex method of work on patients with motor disabilities, be-cause it exacts that conductive teacher -i.e. educator - has a deep trust in the philosophy that is fundamental for this method. For example, "To learn" for Conductive Education is not the simple verb that means "making someone copy prescribed movements". Learning is no "just copying and let himself to lead by the intervention of a teacher". Patients must understand that they are the principal subject that must learn, "abandoning passivity and putting together every fragments" of the actions they are doing.

Results: Conductive Education has given very good results in restoring and improving the disabili-ties of a very large number of in-patients and out-patients in the Petö Institut of Conductive Education of Budapest. With Petö's method a lot of patients with different illness (from ataxia, or diplegia until spina bifida or peripheral paralysis) have been nursed. The new cure have brought them a big relief. Naturally, the cultural skills and medical/pedagogical compe-tences of the conductor - the "conductive educator or conductive teacher" - are fundamental to obtain excellent results with patients of every age.

Discussion: Mária Hári was follower, fellow and helper of the great Dr. András Petö, the Hungarian in-ventor of Conductive Education. Thanks to her patience, power and skills, today we can use a new method of rehabilitation for patients with motor disabilities. The pedagogical method of Conductive Education requires competences that come from different scientific field like medicine, education, philosophy etc.

It is worth the effort of trying to apply conductive pedagogy best practices in different fields of training education for teachers and educators, who will have the responsibility of leading pre-school, school and adult groups in the most difficult situation of their life, i.e. while they are learning to live.

\section{References}

- Hári Mária (1997) A konduktiv pedagogia tortenete, MPANNI, Budapest.

- Balogh Margith (2007) Mária Hári and her Conductive Education, MPANNI, Budapest.

\section{Balance and cyclic rapidity adaptations in children aged from 6 to 11 after proprioceptive or coordinative training}

\author{
F.M. Botti ${ }^{1,2}$, A. Brunetti ${ }^{3}$, M. Procenesi ${ }^{2}$, A. Biscarini $^{2}$, O. Brunetti $^{1,2}$
}

${ }^{1}$ Department of Internal Medicine, Section of Human Physiology, University of Perugia, Italy

${ }^{2}$ LAMS, Perugia, Italy

${ }^{3}$ Department of Orthopaedic, Physical Medicine and Rehabilitation

University of Perugia, Italy

We compared the effect of a proprioceptive or a coordinative training on balance, cyclic rapidity and leg power in 59 children of age between 6 and 11 . They were randomly assigned to a proprioceptive training (13 males, 19 females), or to a coordinative program (14 males 13 females). The training sessions were performed twice a week for two months,. The motor capacities were measured one day before and one after the training period. Balance was evaluated as Center of Foot Pressure velocity (CFPv) in bipodal stance tests with eyes open (EO) and closed (EC). Rapidity was measured as foot contacts during a 15" "run in place" task; force as jump height in squat and countermovement jumps (SJ, CMJ). The results were statistically analyzed for the effects of age, gender, and training, the modifications were assumed significant if $\mathrm{p}<0.05$. Provided that no significant difference was found between males and females, the following results! are reported as mean values and SD independently of gender. In addiction, since proprioceptive training only determined significant effects, the post-training data refer exclusively to this intervention type.

SJ and CMJ, showed an age-related linear increment $(20 \pm 5 \mathrm{~cm}$ at age 6 , $28 \pm 7 \mathrm{~cm}$ at age 11 for CMJ; $16.5 \pm 6 \mathrm{~cm}$ and $26 \pm 5 \mathrm{~cm}$ at age 6 and 11 respectively for SJ). No effect of training was observed on these variables. $\mathrm{CFPv}$ in EC ranged from $1860 \pm 260 \mathrm{~mm} / \mathrm{min}$. at age 6 , to 
$1280 \pm 175 \mathrm{~mm} / \mathrm{min}$. at age 11 . In EO CFPv was $1370 \pm 150 \mathrm{~mm} / \mathrm{min}$. and $1100 \pm 175 \mathrm{~mm} / \mathrm{min}$. at age 6 and 11 respectively. CFPv exhibited an high rate of decrement between age 6 and 9 followed by a flat trend between age 9 and 11, data distribution could be fitted an exponential decay curve. The ratio between EC and EO CFPv (Romberg Quotient RQ) resulted higher (1.25 \pm 0.17$)$ in early ages and decreased to $1.09 \pm 0.15$ at age 11. The proprioceptive training induced a $10 \% \mathrm{CFPv}$ decrease in EC and $5 \%$ in EO. Rapidity values increased linearly with age, the contacts ranged from $27 \pm 8$ to $56 \pm 12$ for age 6 and 11 respectively; a $22 \%$ posttraining increment was observed. In! conclusion beside the linear increments in leg power and in cyclic rapidity, these results evidence a nonlinear profile in the development of balance control that reaches stable performances at age 8-9. As suggested by RQ profile, an increased efficiency in non-visual cues processing may be the substrate of the improved postural performances. The different efficacy between prorprioceptive and cohordinative training may be explained considering that proprioceptive exercises are performed under posturally demanding conditions requiring continuous closed loop corrections, the concomitant intense multisensory self-motion signals may stimulate the adaptation of the control system.

\section{ORAL PRESENTATIONS}

\section{Saturday, October $6^{\text {th }}, 2012$}

\section{Session 5.1. Coaching and Mental Training}

\author{
Self-esteem, body image and B.M.I. in people attending fitness \\ classes
}

M. Alesi ${ }^{1}$, A. Bianco ${ }^{2}$, S. Spina ${ }^{3}$, M. Raccuglia ${ }^{3}$, A. Palma ${ }^{2}$, A. Pepi ${ }^{1}$

${ }^{1}$ Dip. Psicologia, Università di Palermo, Italy

${ }^{2}$ Dip. DISMOT, Università di Palermo, Italy

${ }^{3}$ Laureato in Scienze delle Attività Motorie e Sportive

Over the last decade there have been several studies which support the role that physical exercise plays in the promotion of well-being and positive mental health. A crucial aspect in the well-being is the relationship between self-esteem and body-image. Anyway limited theoreticallybased longitudinal studies on body image and its relationship with selfesteem among adult men and women is documented (Grossbard, Lee, Neighbors, Larimer, 2009; Mellor, Fuller-Tyszkiewicz, McCabe, Ricciardelli, 2010).

Different cultural standards for ideal body types have been identified: for females a thin figure represents attractiveness and for males, a more muscular build symbolizes power and success. Body mass index (MBI) has proven to be the most reliable biological correlate of body image and studies show an association between elevated BMI and greater body dissatisfaction (Milligan, Pritchard, 2006).

Given these theoretical premises, the study aims to investigate the selfesteem and body-image of individuals practicing fitness as a function of gender and age. The study also examines the role of B.M.I. in the relationship between self-esteem and body-image.

The sample consisted of 100 participants (50 females and 50 males), with an average chronological age of 28.59 years (range $18-63$; $\mathrm{SD}=$ $10.2)$, subdivided into three groups of age: Group 1 ( $<20$ years), Group 2 (20-30 years), Group 3 (> 30 years).

At the beginning of the research subjects were given a Fitness Test aimed at evaluating parameters such as weight, tall, sit-up test, push-up test, squat-test... Then were administered the Self-Esteem Scale (Rosenberg, 1965) and the Body Weight, Image and Self-Esteem Evaluation Questionnaire (Awad, 2004) to measure the subjects' self-esteem and body image. Instruments were collectively administered during the regular fitness classes.

In summary, results indicate significant differences between males and females both for Self-Esteem (F 1, $99=6.694 ; \mathrm{p}<.05$ ) and for Body-image $(\mathrm{F} 1,99=11.708 ; \mathrm{p}<.01)$. In particular, males show higher levels of Self-Esteem and Body-image than females. Moreover the results demonstrate an association between B.M.I., Body-Image and Self-Esteem. Additional research should extend the sample with subjects practicing differentiated kinds of sport activities and a control group composed by sedentary subjects.

\section{Aspects of coach-athlete relationship: successful versus unsuccessful outcome in young soccer players}

R. Schembri, S. Pignato, S. Di Stefano, G. La Delfa, S. Nicolosi

Facoltà di Ingegneria, Architettura e Scienze motorie, Università di Enna Kore, Enna, Italy 
The coach-athlete relationship is an important part of the dynamic and complex coaching process (Jowett \& Cockerill, 2002). "Effective relationships include basic ingredients such as empathic understanding, honesty, support, liking, acceptance, responsiveness, friendliness, cooperation, caring, respect and positive regard" (Jowett \& Cockerill, 2003; Jowett \& Meek, 2000).

On the contrary ineffective relationships will bring usually to unsuccessful conclusions, such as lack of interest and positive emotions, not to achieve team goals, deceit, exploitation (Balague, 1999; Brackenridge, 2001; Jowett, 2003). Ames (1992) introduced the term motivational climate to capture all the overriding aspects of the social-psychological environment. The present study aims to analyse the coach-athlete relationship troughout the dispositional achievement goal orientations and the motivational climate in sport for young soccer players.

Our sample is composed by children and teenagers who attend soccer schools. Participants were administered a battery of instruments including:

Task and Ego Orientation in Sport Questionnaire (TEOSQ) by Duda et al. (1995), Italian version by Bortoli and Robazza (2003);

The Perceived Motivational Climate in Sport Questionnaire (PMCSQ-2) by Newton, Duda, and Yin (2000);

The Coach-Athlete Satisfaction Questionnaire by Jowett (2003).

Questionnaires were administered in established soccer schools located in Palermo and Catania (Sicily).

Previous studies showed that the perceived motivational climate and Motivational Orientation in Sport may affect the coach-athlete relationship. The study aimed to confirm the positive correlation among positive motivational climate, task goal orientation and good coach-athletes relationship. We detected positive and negative aspects of the interpersonal relationship and possible behavior consequences.

Levels of anxiety and self-esteem associated to sport activity in university students: a pilot study

G. Rappo, M. Alesi, A. Pepi

Dip. Psicologia, Università di Palermo, Italia

The literature supports the role that physical exercise plays in the promotion of well-being and positive mental health. Unfortunately, while the somatic benefits associated with physical exercise are well documented, such as the long term protection against somatic complaints including coronary heart disease, hypertension, a number of cancers, diabetes, osteoporosis, hard evidence to support an equivalent relation between exercise and psychological well being is quite small. Much of the existing literature on exercise and mental health has focused on changes in anxiety, self-esteem, depression, mood and stress reactivity. A pivotal aspect in the well-being is the relationship between self-esteem and anxiety associated to physical activity (De Moor, Beem, Stubbe, Boomsma, De Geus, 2006).

The current study aims to investigate the relationship between levels of global self-esteem, levels of anxiety of both state and trait, and the beliefs on the sport activities.

The sample consisted of 126 college students, 35 male and 91 female, attending Motor Sciences, Psychology and Architecture university courses. The average chronological age was of 21,5 years.

Students were tested on a whole battery including: 1) the Self-Esteem Scale (Rosenberg, 1965) to measure the global self-esteem; 2) STAY 1 and 2 (Spielberger, Gorsuch, \& Lushene, 1970) to measure anxiety of both state and trait; 3) Questionnaire concerning the beliefs on the sport activities articulated in 12 items.

In summary students attending Motor Sciences, practicing more sport activities than other students, show lower level of anxiety of both trait and state.

Moreover we found a negative correlation between self-esteem and trait anxiety.

These results confirm the role of sport activities in the promotion of mental well-being by reducing levels of anxiety.

\section{Active breaks and attention in primary school pupils: methodological concerns}

\section{A. Carraro, I. Ferri, E. Gobbi}

\section{Dipartimento FISPPA, Università di Padova, Italy}

Background: Children participation in physical activity is still a major concern of health education research. Because of the amount of sedentary time that children spend in classroom every day, the school environment could be a good opportunity to provide adequate levels of physical activity (Pate et al., 2006). Active breaks may occur during physical education, recess, classroom time, or with after-school programs and it has been seen that they positively influenced on-task behavior in children (Mahar, 2011). The purpose of this study was to examine the effect of active breaks on attention during school day in a group of primary school pupils.

Materials and method: Forty-six children in three classrooms of 4th and 5th grade of a school located in Veneto participated in the study. A baseline evaluation of selective attention allows us to select eligible cases for the analysis. Evaluations of attention was conducted independently for each classroom, in three days with different situations. The different conditions randomly proposed to the 3 classrooms consisted of a usual school day, a day with 3 inactive breaks (reading comics) and a day with 3 active breaks. (i.e. doing "energizers", a set of 5 to 8-minute long "movement oriented exercises" developed by Mahar and Colleagues in 2004 to promote physical activity and learning).Children's attention was measured with the Italian version of the Continuous Performance Test (CP, Marzocchi et al., 2010) after the first, the fifth and the eighth school hour. Moreover, children wore an accelerometer (ActiGraph, GT3X+) in order to measure physical activity quantity a! nd intensity.

Results: After the exclusion of 3 children from the analysis, because of their under cutoff baseline attention values, final sample consisted of 21 boys and 22 girls, mean age was 9.5 years $(\mathrm{SD}=0.6)$. RM-ANOVA shown that children's attention was significantly higher by classroom and by condition on the last administration of the school day and on the third condition proposed $(p<0.05)$ independently from its type. Concerning physical activity quantity, children of all classrooms reported significantly higher steps count per school day during active breaks condition $(\mathrm{F}(4,38)=18.71 ; \mathrm{p}<0.01)$, with a mean increment of $55 \%$. Also minutes of moderate to vigorous physical activity during activebreak days reported significantly higher values compared with inactive breaks and normal conditions $(\mathrm{F}(4,38)=12.33 ; \mathrm{p}<0.01)$.

Discussion: According to our experience, some methodological concerns should be considered when investigating the effect of active breaks on attention: attention improvement on the last administration of the $\mathrm{CP}$ test could be consequent to a learning effect; the CP test proposes a problem regarding the compromise speed/accuracy. So, it is suggested to consider alternative measurement approaches, such as collecting data with computer based tests, or adopting direct observation of behaviors. 


\section{ORAL PRESENTATIONS}

Saturday, October $6^{\text {th }}, 2012$

\section{Session 5.1. Adapted Physical Activity}

\author{
Physical fitness in psychiatric patients admitted to a residential \\ treatment
}

\author{
A. Carraro ${ }^{1,2}$, I. Ferri ${ }^{1,2}$, E. Gobbi ${ }^{1}$, A. Galardini ${ }^{2}$, I. Mondelli ${ }^{2}$, \\ A. Fiorellini ${ }^{2}$
}

${ }^{1}$ Department FISPPA, University of Padua, Italy

${ }^{2}$ Casa di Cura Parco dei Tigli, Villa di Teolo, Padova, Italy

Background: Despite exercise has been seen as a cost-effective intervention to increase both physical and psychological conditions and quality of life in people with mental health problems (Faulkner \& Biddle, 2002), there are increasing evidences that people suffering from psychiatric diseases adopt more sedentary behaviors and have less strenuous form of physical activity compared with general population (Faulkner \& Taylor, 2005; Probst \& Carraro, 2012).

The aim of this cross-sectional study was to explore the relations of physical activity habits, BMI and fitness conditions in a group of patients consecutively admitted to a residential multi-dimensional treatment for psychiatric disorders.

Materials and method: Participants were 120 psychiatric patients (men $=44$; women $=76$; mean age $=46.9, \mathrm{SD} \pm 11.1$ years; mean $\mathrm{BMI}=26.8$, $\mathrm{SD} \pm 6.1 \mathrm{~kg} / \mathrm{m} 2), 8$ were diagnosed with psychotic disorders. The group was representative of the population seeking care for mental health problems in residential clinics.

They filled in the IPAQ (International Physical Activity Questionnaire) and participated to a test battery to evaluate walking capacity and upper and lower limbs strength. The 6-minute walking test (6MWT), the handgrip test (HG) and the counter-movement-jump test (CMJ) were administered. Measures were collected during the first days of hospitalization, before patients started with exercise programs. Results were compared with data from general population and with those collected in a psychiatric clinic in Belgium.

Results: According to the IPAQ scores categories, $61.7 \%$ of participants reported low level of physical activity when they were at home, $26.7 \%$ moderate and $11.7 \%$ high level, with no significant differences by gender. Differences by gender resulted in the 6MWT $(\mathrm{p}<0.01)$, in the HG test ( $\mathrm{p}<0.001)$ and in the $\mathrm{CMJ}(\mathrm{p}<0.001)$, with men scoring higher than women. No significant differences in fitness tests were found by IPAQ categories. Significant correlations of the 6MWT with the BMI $(r=-.31$, $\mathrm{p}=0.001)$, the $\mathrm{HG}(\mathrm{r}=-0,49, \mathrm{p}<0.001)$ and the $\mathrm{CMJ}(\mathrm{r}=-0,30, \mathrm{p}=$ 0.001 ) tests were found. Correlates of the 6MWT were included in a stepwise multiple regression analysis, muscular fitness (HG and CMJ) and BMI were identified as independent predictors of the walking capacity. The model explained $64.8 \%$ of the variability.

Discussion: Data from 6MWT, HG and CMJ tests were below the average data reported for general population (Eurofit, 2008) and similar to those reported by patients treated in a psychiatric clinic in Belgium (Vancampfort et al., 2012; Vancampfort, Carraro et al., in press). BMI data highlighted that overweight and obesity represent a serious problem for psychiatric patients. Results underline that psychiatric patients are unfit compared with the general population and that specific exercise programs should be included in the treatment and prevention of mental health problems.
Exercise engagement of people with mental health problems: motivational aspects

A. Carraro, I. Ferri, E. Gobbi

Department FISPPA, University of Padua, Italy

Background: Physical activity (PA) has been recognized to positively affect prevention and treatment of mental disorders (Faulkner \& Biddle, 2002), unfortunately PA levels and fitness of psychiatric patients are usually very low (Vancampfort et al., 2011). Active lifestyle and regular exercise need to be supported by personal motivation, and people with mental diseases are often characterized by lack of motivation and poorer networks that negatively influence personal volition and coping abilities. Recent studies underlined the importance to promote intrinsic motivation to engage individuals in targeted behaviors, stimulating enjoyment and personal satisfaction rather than external reward (Choi et al., 2010).

The aim of this study was to evaluate the effect of a multidimensional approach on motivation, enjoyment and perceived benefits and barriers toward exercise, in a group of psychiatric inpatients.

Materials and Method: Participants were a mixed-gender sample of 53 psychiatric inpatients (men $\mathrm{n}=21$; women $\mathrm{n}=32$; mean age $=47.7$ years, $\mathrm{SD}=10.4$ years; mean $\mathrm{BMI}=26.5, \mathrm{SD}=5.4$ ) attending a multidimensional treatment program including group-based exercise programs. Before to enter the study participants completed the International Physical Activity Questionnaire (IPAQ), $58.5 \%$ of them reported a low level of habitual PA, $20.8 \%$ a moderate and $20,8 \%$ a high PA level. They filled in a package of questionnaires before and at the end of the intervention (corresponding with the discharge from the hospital). The Physical Activity Enjoyment Scale (PACES) was used to assess enjoyment. The Situational Motivation Scale (SIMS) was used to test motivational aspects. Perceived benefits and barriers of PA were investigated with the Decisional Balance Scale (DBS).

Results: At baseline participants did not report significant differences in questionnaires values, neither by gender nor by IPAQ categories. At baseline, significant negative associations were found between BMI and positive scales $(\mathrm{p}<0.01)$, in particular with PACES (pros scores), DBS and SIMS intrinsic motivation and identified regulation subscales. Positive associations were found between BMI and the negative subscales: SIMS amotivation and PACES-cons. Paired ttest shown significant differences in post versus pre scores of SIMS amotivation subscale $(\mathrm{t}(52)=-2.68, \mathrm{p}<0.05)$ and DBS $(\mathrm{t}(52)=3.36$, $\mathrm{p}<0.01)$.

Discussion: Data underlined that high BMI is associated with low level of enjoyment, perceived benefits, and intrinsic motivation toward exercise. Moreover, results suggested that a multidimensional approach, also when corresponding to a short-time intervention (hospitalization reported a 33-day mean length), could improve motivational attitude and the perception of benefits toward exercise among people with mental health problems. Further research is necessary to understanding longterm effect of exercise training by means of follow-up evaluations and objective measure of leisure time PA levels.

\section{A training protocols of transtibial amputee sprinting athlete: a pilot study}

\section{F. Sgrò, P. Mango, D. Riggi, R. Schembri, S. Pignato}

Facoltà di Ingegneria, Architettura e Scienze motorie, Università di Enna Kore, Enna, Italy 
Introduction: Research on training methods of sprinters with unilateral amputation is a field of considerable interest, although the limited literature is due to the small number of cases. The present study aims to carry out a pilot project focused on training techniques for development of the strength, a conditional skill essential for a sprinter with unilateral trans-tibial amputation, ICP class T44 (Bertini, 2005).

Method: The project refers to a specific methodology for development of the force. The analysis of sprinting demonstrate that athletes using dedicated sprinting prosthesis have a lower reaction strenght in the prosthesic limb than the healthy one.

In particular, the differences concern the symmetry of the propulsive thrusts in the two arts; a higher work of compensation of the hip joint prosthetic limb, due to the absence of the propulsive plantar flexion of the mechanical foot; the inferiority of the angle flexion of the knee. In the case of short stumps, in fact, the knee flexion is less than 20-30 degrees.

The athlete participated in a preliminary training of the force performing both under natural loads and in a gym, in two-hours daily sessions. In a following step they have made a strengthening training, using weightlifting exercises.

Conclusions: The training of an athlete using dedicated sprinting prosthese is centered on the development of force to $60 \%$, the speed for $20 \%$, and the resistance to $20 \%$. The muscular capacity is particularly important in the training process. Strength training is crucial for the sprinter, as it assumes the starting sprint and the subsequent acceleration skill, which can not occur if legs do not have enough strength. To understand how we can develop strength were applied, in particular, two techniques of training: Endurance to the Maximum Speed and Lactacid Power (C. Vittori, 2004).

The plan includes 3 weeks of training load, 1 week of training unload, and 6 training each weekend. Each training is divided into a warming-up phase (mild stroke, active and passive stretching, and joint mobility exercises) a technical rate phase, and a cool-down phase.

\section{ORAL PRESENTATIONS}

\section{Saturday, October $6^{\text {th }}, 2012$}

\section{Session 5.2. Training and Performance}

\author{
Development of core stability in young female athletes \\ of synchronized swimming
}

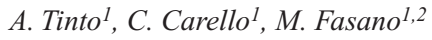 \\ ${ }^{1}$ S.U.I.S.M. - School of Physical and Sports Education - University \\ of Torino, Italy \\ ${ }^{2}$ Italian Swimming Federation
}

Aim: The problem encountered by coaches and trainers concerns the difficulty of controlling one's body during specific technical figures performed during progressions in water, in particular keeping the trunk in an inverted vertical position, basic element of this sport.

The test objective is the creation of a working protocol (alternative) aimed at the development of core stability, body section fundamental for technical elements of this sport

Methods: The sample group consisted of 20 female agonist athletes, age $10 \pm 1 \mathrm{yrs}$, weight $26 \pm 4 \mathrm{Kg}$, height $153 \pm 6 \mathrm{~cm}$. The group of athletes, who belong to the same sports club, who have the same anthropometric measurements and have followed the same training methodology, were divided into two groups, the control group (CG) and work group (EG). The control group has performed a normal training, whereas the work group performed a series of exercises prepared and studied by the coachs staff.

TRX which has already been in use for some years for the training of athletes with specific exercises on the ground, was used as research tool. This tool (TRX) is used to make dry exercises very similar to the model of exercises that are performed in water.

The total duration of the work was six months, with two training sessions a week, with each session lasting about 15 minutes. The protocol proposed included 5 exercises, characterized by different application procedures.

Results: Both groups underwent two assessments: one at the beginning (T0) prior to the commencement of the work, and one (T1) at the end of the experiment. The assessment tests are focused on the abdominal area, mainly transversus abdominis and obliques, since they are the main stabilizers of the core region. The Stabilizer was used (facing down and on one's back) during the first two tests. It is a pressure device used during reinforcement rehabilitation exercises. The third test was devised by McGill and evaluates ability to keep the trunk stable by means of physical resistance tests.

The analysis of the results suggests that both groups showed improvements, which were more evident in the work group. The statistical analysis (performed with PAIRED test, Wilcoxon Test and Mann-Whitney U test), revealed significant differences between the two groups in almost all of the tests.

Results: Supine stabilizer (significant differences for $\mathrm{EG}$ with $\mathrm{P}<0.0001$; non-significant differences for $\mathrm{CG}$ with $\mathrm{P}=0.1396$ ); Prone stabilizer (significant differences for $\mathrm{EG}$ with $\mathrm{P}<0.0001$; non-significant differences for $\mathrm{CG}$ with $\mathrm{P}=0.1482$ ); McGill test (significant differences for $\mathrm{EG}$ with $\mathrm{P}<0.0001$; non-significant differences for $\mathrm{CG}$ with $\mathrm{P}=0.1396$ ).

\section{Conclusions:}

The test results confirm the validity of the work method added to the training program. 


\section{Acute effects of static and dynamic stretching on explosive strength performance in basketball players}

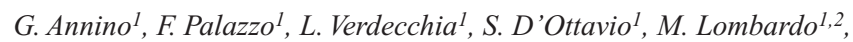
R. La Torre ${ }^{l}$, V. Tancredi ${ }^{l}$, E. Padua ${ }^{1,2,3}$

${ }^{1}$ Corso di laurea in Scienze Motorie, Facoltà di Medicina e Chirurgia, Università degli studi di Roma "Tor Vergata", Italia

${ }^{2}$ Facoltà di Scienze Motorie, Università Telematica San Raffaele Roma, Italia

${ }^{3}$ Scuola dello Sport, Coni-Sicilia, Italia

In the last decade negative effects of static stretching (SS) on explosive strength performance has been reported by many studies and dynamic stretching (DS) has been recommended to be more suitable as a part of warm-up (1). Almost all studies focused on the explosive strength performed immediately after the stretching exercises $(1,2,3)$, whereas in both training and competitions usually the stretching phase always follows a period of 10-15 minutes of basketball warm-up at increasing intensity and sport skills specificity. Aim of this study was to monitor the explosive strength performance before, immediately and after administration of a static stretching protocol (SSP), a dynamic stretching protocol (DSP) and after a sport specific pre-game basketball shooting phase of $15 \mathrm{~min}$.

Nine players (age $29 \pm 6.73$, height $192.67 \pm 7.75$, and weight $88 \pm$ 8.17) of a basketball team participating to the 4th Federal League National Level participated to this study. The cross-over method was adopted considering the low numbers of subjects. Players were randomly divided in two groups. Static (SS) and dynamic (DS) stretching protocols were administered in inverse order. Tests were performed weekly in the first day of two consecutively micro cycles. Experimental cross over design was 10 minutes of warm-up, SS, DS, $15 \mathrm{~min}$ of pregame basketball warm-up and jump test at the end. Four lower limbs muscles exercise of a partial duration of 40 s for each position and leg for a total duration of $8 \mathrm{~min}$ were included in both stretching protocol. The one-way repeated measures ANOVA ( level of $p<0.05$ ) were used to determine the effects of the different stretching protocol on jump performance.

Results of this study shows that SS potential decrease disappears after an enough long period of neuromuscular reactivation. Same increase in jump performance has been observed in DS protocol. Therefore, $8 \mathrm{~min}$ of static and/or dynamic stretching protocol followed by a sport specific pre-game shooting warm-up phase does not influence the neuromuscular performance before the beginning of the basketball practise session and/or competition.

\section{References}

1. Carvalho FL, Carvalho MC, Simão R, Gomes TM, Costa PB, Neto LB, Carvalho RL, Dantas EH (2011) Acute effects of a warm-up including active, passive, and dynamic stretching on vertical jump performance. JSCR

2. Cramer, J.T., Housh, T.J., Weir, J.P., Johnson, G.O., Coburn, J.W., Beck, T.W (2005) The acute effects of static stretching on peak torque, mean power output, electromyography, and mechanomyography. EJAP, 93, 530-539

3. Kokkonen J., Nelson A.G.,Cornwell A (1998) Acute muscle stretching inhibits maximal strength performance. RQES , 69,411-415

\section{Analysis of the correlation between core stability and performance}

\section{Dalla Libera, G. Coratella, F. Schena}

${ }^{1}$ Dip. Scienze Neurologiche, Neuropsicologiche, Morfologiche e Motorie, Università di Verona, Italia
It is known that core ability is involved in power exercises, allowing strength transfer from trunk to distal limbs (Sharrock, 20111). The aim of the study was to identify whether core stability can influence sports performance.

Fifty healthy students ( 30 males and 20 females; age: $20.6 \pm 1.4$ years; height $174.8 \pm 8.6 \mathrm{~cm}$; weight $68.1 \pm 13.4 \mathrm{~kg}$ ) performed twice all the tests. The independent variables were times of McGill test's maximal isometric endurance positions (MG): Back Extension (BE), Trunk Flexion (TF), Right Bridge (RB) and Left Bridge (LB) (Nesser 20082). The dependent variables were Standing Long Jump (SLJ), Star Excursion Balance Test (SEBT), Medicine Ball Throw with crossed legs, in standing and in dynamics (MBT1, MBT2, MBT3) and Accuracy of a tennis ball Throwing, executed with previous described modalities (AT1, AT2, AT3).

Statistical software (SPSS 16.0; Chicago, ILL) was used to perform statistical analyses. In order to test measurements reliability, Intra Class Coefficient (Cronbach ) was adopted with each parameter. Relationships between dependent and independent variables, as well as inter dependent variables were determined using multiple bivariate correlations, represented by Pearson correlation coefficient. Statistical significance was set at $\mathrm{p} \leq 0.05$.

McGill average ICC ranged from 0.880 to 0.961 (CI 95\%). Significant correlations were found between RB and SLJ ( $\mathrm{r}=0.440)$, MTB1 $(\mathrm{r}=0.508)$, MBT2 $(\mathrm{r}=0.495)$, MBT3 $(\mathrm{r}=0.473)$ and AT3, considering simultaneously both sprint time and the score of the launch $(\mathrm{r}=-0.288)$; between LB and SLJ $(r=0.533)$, the three modalities to execute the MBT $(\mathrm{r}=0.504, \mathrm{r}=0.481, \mathrm{r}=0.489)$ and AT3 $(\mathrm{r}=-0.281)$; finally, between $\mathrm{MG}$ and SLJ $(r=0.290)$, and MBT1 $(r=0.325)$.

In the light of the data we obtained, it is possible to affirme that core stabilizer muscles are significantly involved in long jump, throwing and toss accuracy in dynamic conditions, typical team sports tasks.

- Sharrock C et al. 1, "A pilot study of core stability and athletic performance: is there a relationship?", Int J Sport Phys Ther, 2011, 6(2): 63-74

- Nesser TW et al. 2, "The relationship between core stability and performance in Division I football players", J Strength Cond Res, 2008, 22(6): $1750-54$

\section{The effect of the step frequency manipulation on the step length in Race Walkers}

\author{
S. Vando ${ }^{1}$, J. Padulo ${ }^{1,2}$ \\ ${ }^{1}$ Faculty of Medicine and Surgery, University of "Tor Vergata" Rome, \\ Italy \\ ${ }^{2}$ CONI - Italian Regional Olympic Committee, Sardinia, Cagliari, \\ Italy
}

The present study investigated the effects of step frequency manipulation during training in slopes $(2 \%)$ on biomechanical parameters at IsoEfficiency Speed (IES). Twelve male race walkers were randomly allocated to one of two training groups for three weeks: step frequency manipulation group (RWM, n=6) and free step frequency group (RWF, $\mathrm{n}=6$ ). The RWM performed with 5 sets of 5 minutes race walking $2 \%$ slope at IES with a manipulated step frequency (calculated in order to replicate the same step length elicited during level) while in RWM the step frequency was freely chosen. ANOVA with repeated measures and Bonferroni post hoc showed significant differences between both training groups $\mathrm{F}(1,10)=32.856, \mathrm{p}<0.0001(2=0.767)$ respect to baseline condition. In RWM group showed a increased step length $4.54 \%$ (p < $0.0001)$, contact time $4.58 \%(\mathrm{p}<0.001)$ and decreased step frequency $4.44 \%$ ( $p<0.0001)$. Conversely, in RWF the effect of the training has showed a decreased in step length $1.18 \%(\mathrm{p}<0.0001)$, contact time 
$<1 \%(\mathrm{p}<0.0001)$ and increased step frequency $1.19 \%(\mathrm{p}<0.0001)$. These findings coincide with characteristics of better race walking performances. The results from the study could help coaches to device training methods which could improve an athlete's performance through increasing step length. The method provided may aid faster race times for athlete's.

\section{Anaerobic tests on rowing ergometer in young rowers}

\section{A. Cataldo, D. Cerasola, D. Bellavia, G. Russo, D. Zangla, M. Traina}

Dip. DISMOT, Università di Palermo, Italy

Purpose: Performance over $2000 \mathrm{~m}$ on a rowing ergometer is dependent upon the functional capacity of both the aerobic and anaerobic energy pathway. The purpose of this study was to assess the relationship between anaerobic power and indoor performance over $2000 \mathrm{~m}$ in youth rowers. Methods: Six youth rowers, 3 males and 3 females mean age 14 (range $13-15$ ), performed, on a separate occasion, two maximal ergometer power tests, on 20 " and on 60 ", and a maximal $2000 \mathrm{~m}$ ergometer time trial. All evaluations were performed on a Concept II model D air braked rowing ergometer (Concept2, Nottingham, UK). Average speed over $2000 \mathrm{~m}$ (V) and average power over 20" (W20) and over 60" (W60) were measured. The data is shown as mean values \pm standard error of the mean. Pearson's product-moment correlation and stepwise regression analysis were employed to examine the relationship between V, W20 and W60.

Results: The average of rowing speed over $2000 \mathrm{~m}$ was $4,26 \pm 0,17 \mathrm{~m} / \mathrm{s}$. The average power over 20" and over 60 " was respectively $366 \pm 46,94$ $\mathrm{W}$ and $353 \pm 41,35 \mathrm{~W}$. Significant correlations were observed between V and W20 $(r=0,95 \mathrm{p}<0.0005)$ and $\mathrm{W} 60(\mathrm{r}=0,98 \mathrm{p}<0.0005)$. Stepwise multiple regression identifies W20 and W60 as determinants of performance over $2000 \mathrm{~m}$ on a rowing ergometer, accounting for $96.80 \%$ of the variance, $\mathrm{P}<0.01:$ Speed $=2,795-(0,0005303 * \mathrm{~W} 20)+(0,004680 *$ W60). In order to use the model to predict actual performance over $2000 \mathrm{~m}$ on the ergometer, we cross-validated the prediction equation with an independent sample of young rowers. The results of the cross-validation showed that speed over $2000 \mathrm{~m}$ was predicted between limits from 0,12 to $0,11 \mathrm{~m} / \mathrm{s}(-2,74 \%$ to $2,78 \%)$ and demonstrated an extremely significant correlation between actual and predicted rowing speed $(\mathrm{r}=0,98$, $\mathrm{P}=0,0005)$.

Conclusions: The data of our study indicates that, in young rowers, anaerobic power tests correlate significantly with performance, expressed in speed over $2000 \mathrm{~m}$. Our model of the determinants of rowing ergometer speed over $2000 \mathrm{~m}$, based on W20, and W60, may predict actual performance on young rowers.

\section{Incidence of bodily communication in volleyball}

\section{P. A. Di Tore, G. Raiola}

DISUFF - Dept Human, Phylosophycal and Educational Sciences, University of Salerno, Italy

Currently, the process of training, its organization, and teaching methodology need more knowledge on the qualitative aspects of sports performance (Schmidt \& Wrisberg, 2008), including bodily communication. Volleyball is particularly suited to the study of bodily communication for the peculiarity of very limited time available in the neuropsychological processes in the mandatory mode of ball rejecting. (Gaetano Raiola \& Di Tore, 2012)

Focus of the study is to verify the incidence of performance analysis da- ta of bodily communication in volleyball. The sample was chosen to represent the scores obtained in three technical skills strongly influenced by bodily communication:

- The second ball goes to the opposite court instead of setting for attacking;

- The attack as fast as possible in the middle of the net;

- The off speed hit instead of power spike over the block.

The scientific aims of this research work is to analyse three specific skills relating the attacker role and the setter one in relationship to defenders, according to the principles of bodily communication (Argyle, 1988), the aspects of perceptive senses (Berthoz, 2000) and the neurobiological implications (Rizzolatti \& Sinigaglia, 2006) and to give a general plan for training that includes knowledge about the function of gestures, signs and mimicking.

The analysis is carried out during the match, always in contrast to the opponent wall, according to performance analysis techniques. The method used is the recruitment of the data with the use of Data Project software. The assignment of the data is attributed by the team's official analyst after sharing with the coach and the athlete. The data recruited were compared with data on total points in the set and with the overall results of the attack, first individually and then together, in order to determine the relationship among points attributable to bodily communication and the final score.

The results show as this study can help the coach to train the team for improving the analysed technical skills in different mode (G. Raiola, 2012), creating a methodological system training to enhance the performance.

References

- Argyle, M. (1988). Bodily communication: Methuen.

- Berthoz, A. (2000). The Brain's Sense of Movement: Harvard University Press.

- Raiola, G. (2012). Didactics of volleyball into the educate program for coaches/trainers/techniciansof Italian Federation of Volleyball (FIPAV). Journal of Physical Education and Sport, 12(1).

- Raiola, G., \& Di Tore, P. A. (2012). Statistical study on bodily communication skills in volleyball to improve teaching methods (Vol. 7).

- Rizzolatti, G., \& Sinigaglia, C. (2006). So quel che fai: il cervello che agisce ei neuroni specchio: R. Cortina ed.

- Schmidt, R. A., \& Wrisberg, C. A. (2008). Motor learning and performance: a situation-based learning approach: Human Kinetics Publishers.

\section{Body composition predictors of ball-throwing velocity in handball}

\author{
F. Piscitelli ${ }^{1,2,3}$, C. $_{\text {Milanese }}^{1,2}$, V. Cavedon $^{1,2,3}$, C. Zancanaro $^{1,2}$ \\ ${ }^{1}$ Faculty of Motor Sciences, University of Verona, Italy \\ ${ }^{2}$ Department of Neurological, Neuropsychological, Morphological \\ and Movement Sciences, University of Verona, Italy \\ ${ }^{3}$ Doctoral program in Multimodal Imaging in Biomedicine, University \\ of Verona, Italy
}

Ball-throwing velocity is a relevant factor in handball. While velocity depends importantly on an overarm throw requiring specific training and skills, prediction of ball velocity is a major concern for both coaches and athletes.

In this study we investigated the body composition predictors of ballthrowing velocity in male and female handball players.

Forty-six handball players (age 21.2 $\pm 4.16 \mathrm{y}$; stature $172.9 \pm 9.46 \mathrm{~cm}$; body mass $71.1 \pm 15.08 \mathrm{~kg}$; BMI $23.6 \pm 3.40 \mathrm{~kg} / \mathrm{m} 2 ; 22$ males and 24 females) participated in this study after signed informed consent. Ball velocity during an overarm throw in a standing position (as for penalty throws) was measured using a Bushnell Doppler-radar gun. Athletes were asked to throw the ball at $0.5 \times 0.5 \mathrm{~m}$ target located in the right or left upper 
corner of a standard handball goal located $7 \mathrm{~m}$ away. Body composition was measured with dual-energy X-ray absorptiometry (DXA). Linear regression analysis was used to identify predictors of ball velocity. Mean ball velocity was $19.2 \pm 4.77$ and $18.9 \pm \mathrm{m} / \mathrm{s}$ at the right and left target, respectively.

Standard linear regression analysis showed that using \%fat mass and fat-free soft tissue mass as the independent variables the resulting equation had a coefficient of determination (adjusted R2) of 0.783 for ball velocity at both the right and left target. Using the same parameters at the regional level (right arm \% fat mass and fat-free soft tissue mass) the coefficient improved slightly to 0.793 and 0.798 for ball velocity at the right and left target, respectively. The standard deviation of the estimate ranged $1.9-2.2 \mathrm{~m} / \mathrm{s}$. For all equations the variance inflation factor was between 1.2 and 1.7, therefore the models were not violated.

These results show that DXA analysis yields data enabling prediction of ball-throwing velocity in handball with reasonable accuracy.

\section{Rhythmic Gymnastics: an anthropometric approach}

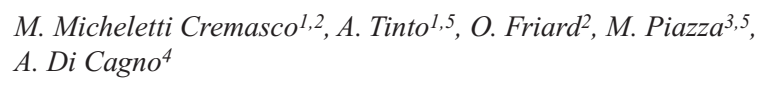

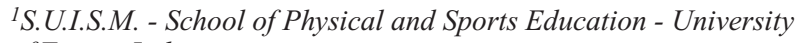
of Torino, Italy

${ }^{2}$ Department of Life Sciences and Systems Biology - University of Turin, Italy

${ }^{3}$ Department of Anatomy, Histology and Forensic Medicine, University of Florence, Italy

${ }^{4}$ I.U.S.M.- Universitary Institute of Motor Sciences - Rome, Italy

Italian Gymnastics Federation

The aim of this study is to describe anthropometric characteristics of elite athletes in rhythmic gymnastics and to evaluate if dimensional parameters have an effect on the success level in this sport. As frequently confirmed in the literature the rhythmic athletes generally respect a specific anthropometric profile required by the sport (Di Cagno et al., 2008; Douda et al., 2008; Gonçalves et al. 2010) but we would like to evaluate if the higher level athletes differ further compared to other athletes, however, referred to a high competitive level.

Methods: The data refer to research carried out during XXIV European Rhythmic Gymnastcs Championships (Torino 2008) consisting of 139 athletes (Junior and Senior) who competed in different competitions (Italian Gymnastics Federation, 2010). We selected 61 athletes as homogeneous sample by age, agonistic level and type of competition (all were exclusively individualist) to evaluate if, among all, athletes finalist have particular anthropometrical differences. Anthropometric measurements here considered were: height, weight and the calculated body mass index (BMI), fat mass, arms span; wrist, thigh, hips, chest and abdomen circumferences. The best score obtained by the gymnasts in the race was used to select the sample (therefore the finalists) and so to compare their dimensional characteristics with the rest of the sample.

Results: The 16 finalists tend to have anthropometric measures more contained for most of the variables, in comparison with 45 non-finalist, while they have a higher stature and wider arms span. In particular comparing the variances of two samples and evaluating the difference by TestF the best athletes result significantly higher and lighter, lower abdomen and thigh circumferences (F 0,03 and 0,04); while the difference is not significant about BMI, wrist, hips and chest circumferences ( $\mathrm{F}$ between 0,1 e 0,2$)$ and even less about the arms span. $(\mathrm{F}=0,4)$.

Conclusion: Seems to be a relationship between body measurements and physical performance (in term of competitive success) our study suggests a positive predisposition referable to higher stature and less weight but not so much to determine a relapse on BMI. Arms span and most of the body circumferences seem to be not relevant. These results are limited to junior sample but we will extend the study to all the rhythmic gymnasts with this "anthropometric measures/competitive success" approach evaluating athletes of different ages and different race specializations to confirm whether or not the assumptions made.

\section{Match analysis of basketball fast break actions: Italian vs Spanish National Team during Eurobasket 2011}

\section{Russo, F. Cairo}

Human Movement and Sport Science Faculty, L'Aquila, Italy

Introduction: Coaches use commonly notational analysis strategies to get physiological and technical information from matches (Barris 2008). Few data are available about fast break strategies both in international and national competitions (Russo 2010), and no data are present on the effects of a made or suffered fast break on offensive and defensive strategy. Aim of this study is to compare the offensive and defensive behaviors of Italian and Spanish National Team after making of suffering a fast break.

Methods: Matches from male Eurobasket 2011 were analyzed for the study: 5 for the Italian National Team and 5 for the Spanish one. The notational analysis for event recording (van der Mars 1989) was carried on through an iPad2 with the Dartfish EasyTag application. Number of actions and number of fast break for each game, causes of starting and ending of each fast break and its efficacy, analysis of the three following offensive and defensive actions after each fast break were measured during each game. Kolmogorov-Smirnov, ICC and T-Test were used for statistical analysis.

Results: There was no significant difference between Italy and Spain both for average number of offensive and defensive actions during a game and for the distribution of fast break during a game. Italy played $5 \%$ of offensive actions with fast break, while Spain $8 \%$. The causes of starting of each fast break were very similar between Italy and Spain. Italy showed great skills in catching the ball because a $79 \%$ of total fast break started from a turnover, while Spain showed a bigger attitude to fast break because a $6 \%$ of actions started from a baseline throw. After a fast break positively scored Italy and Spain showed a trend to fail the following three offensive actions and a trend to play good for Italy and to play bad for Spain in defense during the following three defensive actions. After a suffered fast break scored by the opponent there was a trend both for Italy and Spain to play good the following three offensive actions but to play not very well the following three defensive actions.

Discussion: Results suggest that fast break actions are not discriminating factors between a low level team and an European Champion team. At the same time it is doubtless that playing an offensive of defensive fast break action has got some kind of effects about the strategy adopted for the following actions both in attack and in defense. It is an innovative element that a coach must take into consideration during a match analysis protocol. A larger sample of matches and a longer follow up could confirm this point of view.

\section{References}

1. Barris S. et al. (2008) Sports Med, 38(12):1025-1043

2. Russo L. et al. (2010) J Sports Med Phys Fitness, Suppl 1 50(3):21-22

3. van der Mars H. in Darst PW et al. (1989) IL Champaign Human Kinetics

Relationships between Cooper Test and competition distances $(1000 \mathrm{~m})$ among young track and field athletes

G. Russo, P. Trevisson, F.A. Faure Rolland 
Scuola Universitaria Interfacoltà in Scienze Motorie, University of Turin, Italy

Aim: It is important among young track and field athletes to know about time and space of different distances of running matters in order to have a better knowledge of practice and training procedures; this is also important to know of what to expect from the performances of the single athletes. Through two tests we tried to investigate on which relationships we can find between an encoded test (Cooper Test: TC) and a distance of a typical competition of track and field (1000 m: 1000).

Methods: 23 subjects, 9 females and 14 males of age between 13-14 years $(14 \pm 1)$ involved with track and field have run two tests in a week: a timed run on $1000 \mathrm{~m}(1000)$ and a Cooper Test (TC). After the two run tests we have gotten the following times and distances.

Analysis: The subjects have shown an average result in the TC: $2431 \pm 216 \mathrm{~m}$, in the 1000: $253,8 \pm 27,9 \mathrm{~s}$. The female subjects have shown lower quality results compared to the male subjects in the TC $(2281 \pm 176$ $\mathrm{m} v s 2527 \pm 186 \mathrm{~m})$ and in the $1000(273,7 \pm 31,2 \mathrm{~s} v s 241,1 \pm 16,4 \mathrm{~s})$; the results shown by subjects younger than 13 years old were also lower (TC: $2396 \pm 159 \mathrm{~m}$ vs $2462 \pm 262 \mathrm{~m} ; 1000: 256,4 \pm 28,2 \mathrm{~s} v s 251,5 \pm 28,6 \mathrm{~s})$. The average speed of run is shown to be lower in the TC $(-0,61 \pm 0,18 \mathrm{~m} / \mathrm{s})$; the decrease of speed in the female group is lower $0,52 \pm 0,20 \mathrm{~m} / \mathrm{s} v s$ $0,66 \pm 0,14 \mathrm{~m} / \mathrm{s}$ ), whereas among different ages the results are similar

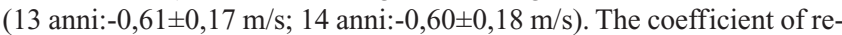
gression of speed, related to the logarithmic function $y=a \log (x)+b$ generated based on the speed run on 1000 and in the TC (Ranucci, Miserocchi, 1985), has an average "a" $=-2,45$. The variation of the average speed of the two tests is not statistically significant in matter subjects' age and sex (Mann-Whitney Analysis alpha $>0,05$ ). The analysis of the correlation with the Test Spearman has shown how the average speed of the two run tests are related $(\mathrm{r}=0,89 ; \mathrm{p}=0,001)$, while the coefficient " $\mathrm{a}$ " and the results of the TC are not related $(\mathrm{r}=0,068)$.

Results: The differences recorded in the TC referred to sex and age of the subjects are within the parameters shown by the authors (males vs females: $+200 \mathrm{~m}$; $14 \mathrm{vs} 13$ years old: $+50 \mathrm{~m}$ - Grosser, Bruggemann, Zitl 1986, in Weineck 2007). According to the exsisting relationships between VO2max and anaerobic threshold (Yoshita T, Chida M, Ichioka M, Suda Y, 1987) the scarce correlation between the coefficient"a" and the results of TC, in contrast with what enounced by Ranucci, Miserocchi 1985 confirms how in between the youngsters between 11 and 18 the parameters VO2max e TC are not related (Apor 1988, in Weineck 2007). The high correlation between the results in the TC and 1000 could indicate how the two tests demand similar energy processes from the examined subjects. The TC is a good indicator of the qualities that might be shown by on the 1000 meters distance for youngsters of age 13-14 of both sexes, but it would not show predictable parameters in the $1000 \mathrm{~m}$.

\section{Circadian variations of physical capacity in Greek Roman wrestlers}

\section{Gollin, A. Guerra}

Motor Science Research Center, University School of Exercise \& Sport Sciences, University of Turin, Italy

Aim: The research investigated, in a group of Greek-Roman wrestlers, whether the flexibility of the back and the hamstrings (MTF), the maximum strength of the forearms (HG) and the explosive elastic strength of the lower limbs are affected by circadian modifications comparing a specific training session at 10 a.m. (and one at 6 p.m.

Methods: Ten Greek-Roman wrestlers competing at national level (age
$19 \pm 3$ years, weight $76 \pm 14 \mathrm{~kg}$, height $176 \pm 10 \mathrm{~cm}$ and $7 \pm 3$ years of training) were involved. The measurements were performed at specific times: before warm-up (Pre-W); after warm-up (Post-W); after technical training (Post-At); after combat phase (Post-C); after cool-down (Post-D).

Results: Evening training (ET): In the standing long jump test (SLF) the results were: 1) Pre-W $v s$ Post-At (Friedman test, $\mathrm{P}<0.01$, post-hoc, $\mathrm{P}$ $<0.01,+8 \%$ ), 2) Pre-W vs Post-C (Friedman test, $\mathrm{P}<0.01, \mathrm{P}<0.01,+$ $9 \%)$.

Morning training (MT): in the standing long jump test the same results were obtained as in the evening: 1) Pre-W vs Post-At (Friedman test, $\mathrm{P}$ $<0.01$, post-hoc, $\mathrm{P}<0.01,+8 \%)$, 2) Pre-W vs Post-C $(\mathrm{P}<0.01,+9 \%)$. In the seat and reach test the results was: 1) Pre-W vs Post-C (Friedman test, $\mathrm{P}<0.01$, post-hoc, $\mathrm{P}<0,01,+15 \%$ ); 2 ) Pre-W vs Post-D (Friedman test, $\mathrm{P}$ $<0.01$, post-hoc, $\mathrm{P}<0,01,+13 \%)$. The comparison between ET and MT (Wilcoxon test) were: 1) Pre-W, SLF (ns ,-1,8\%), FMT (ns ,-8,5\%) and HG (ns, -6,4\%); 2) Post-W, SLF (ns, -2,5\%), FMT (ns, - -0,4\%) and HG (ns, $+0,3 \%$ ); 3 ) Post-At, SLF (ns, $-2,4 \%$ ), FMT (ns, $-2,5 \%$ ) and HG (ns , $-10,9 \%)$; 4) Post-C, SLF (ns , $-1,7 \%)$, FMT (ns, $+2,9 \%)$, HG ( $<<0,01$, $-12 \%)$; 5) Post-D, SLF (ns, $-1,6 \%$ ), FMT (ns, $+0,2 \%$ ) and HG (ns, $7,5 \%)$.

Discussion/Conclusion: In the HG the result indicates that athletes have to train at different times of the day to adapt to the competition time. The variation in FMT during MT stresses the importance of pre-competition warm-up.

\section{Body composition in professional and amateurs rugby players}

\section{F. Spiga, P. Brasili, S. Toselli}

Dipartimento di Biologia Evoluzionistica e Sperimentale, Università di Bologna, Italy

In rugby there is a close relationship between body size of athletes, body composition and sporting success, both individually and as a team. The determination of anthropometric characteristics may then become an important instrument for the selection of athletes by managers and coaches.

The analysis presented here aims to investigate the variability of body composition in athletes of professional and amateur level, taking into account the differences determined by role played by players. The results are evaluated considering also frequency and duration of training sessions conducted weekly by professionals and by amateurs.

The sample consists of 66 athletes, aged between 18 and 35 years. Professional athletes are 38 (28 Forwards and 10 Backs) and amateur athletes 28 (17 Forwards and 11 Backs).

The anthropometric characteristics presented here are height, weight and those that are useful to calculate body composition (skifolds: triceps, pectoral, axillary, subscapular, suprailiac, abdominal and thigh) using the equations of Jackson \& Pollock (1978), suggested as valid for athletes (Sinning 1996), and Siri.

Through a questionnaire has been possible to determine number and hours of weekly training.

Professional athletes show higher heights, less body fat and more muscular mass in comparison with amateur athletes. The differences between the roles denote higher values of height, weight, lean mass and also fat mass in forwards than backs.

In conclusion, we can detect a similar trend in anthropometric characteristics and body composition in the comparison of roles in the two grade levels.

These differences are more pronounced in professional athletes suggesting a guideline in considering the character issues and selective for the success of the discovery of new talents. 


\section{Karate vs. karate: differences between kata and kumite in word champions karateka}

\author{
F. Quinzi, V. Camomilla, P. Sbriccoli
}

Dip. Scienze del Movimento Umano e dello Sport-DISMUS. Università di Roma " Foro Italico", Roma, Italy

Introduction: Karate competitions are divided in combat (Kumite) and forms (Kata). The two competitions present a common technical background. Nevertheless, differences might be present between the techniques performed during Kumite and Kata due to the different demands of the two competitions. Among the various techniques, in karate, the Roundhouse kick (RK) is one of the simplest and it is taught from the very beginning of karate practice. The aim of the present study is to characterize from a neuromuscular point of view two athletes competing in Kumite and Kata while performing a RK.

Methods: Two male karateka competing in Kumite and Kata participated in this study. Both athletes were former world championship winner. Each athlete performed 20 RK continuously. Muscle activity of Vastus Lateralis (VL) and Biceps Femoris (BF) was assessed by means of a portable sEMG recorder (BTS-pocket EMG) in bipolar mode. Electrodes were placed in accordance with SENIAM (Hermens, 2000). Kicking leg kinematics was recorded with a stereophotogrammetric system (Vicon) operating at $100 \mathrm{~Hz}$. Marker placement was carried out according to ISB recommendations (Wu et al., 2002)

Kicking leg kinematics was obtained according to Grood and Suntay (1983), knee angular velocity was derived from the kinematics on the sagittal plane.

For each kick of the sequence, knee peak angular velocity during flexion (AV-fle) and extension (AV-ext) were detected using a custom written algorithm.

For each kick, the time interval ranging from the beginning of knee flexion to the end of knee flexion identified the loading phase (LP); the kicking phase (KP) was considered as the interval between the end of knee flexion to the end of knee extension.

During each phase of each kick, for VL and BF, the average rectified value (ARV) was computed.

For VL and BF Agonist (AGO) and Antagonist (ANT) muscle activity during each phase were obtained by normalizing the ARV obtained as antagonist or agonist to the maximal ARV obtained during the kick.

For each subject the average value among all kicks of the sequence was computed for AV-fle, AV-ext, VL-AGO, BF-AGO, VL-ANT, BF-ANT. Mann-Whitney $U$ test was performed to test for differences between the subjects in all the above-mentioned parameters. Significance level was set to 0.05 .

Results: The Kata athlete demonstrated higher AV-fle $(670 \pm 76 \%$ s vs 539 $\pm 101 \%$ s; $\mathrm{p}=.0002) ;$ AV-ext $(1808 \pm 172 \%$ s vs $1670 \pm 35 \% \mathrm{~s} ; \mathrm{p}=.0004) ;$ BF-AGO $(62 \pm 14 \%$ vs $19 \pm 5 \%$; $<.0001)$. No difference emerged in VL-ANT (12 $\pm 7 \%$ vs $21 \pm 17 \%$; $=.0548)$, BF-ANT (51 $\pm 21 \%$ vs 62 $\pm 18 \% ; \mathrm{p}=.0834)$ and VL-AGO $(54 \pm 24 \%$ vs $45 \pm 28 \% ; \mathrm{p}=.1636)$.

Discussion: A different strategy seems to be adapted by these two athletes in the LP. In particular, the Kata athlete presents a more active knee flexion (BF-AGO), this aspect, coupled to the similar VL-ANT, seems to account for the higher AV-fle. During the KP a similar strategy between the subjects emerged.

\section{Exercise Intensity and inflammation markers during an ultra-cycling event: a case study}

\author{
D. Nonis ${ }^{1}$, A. La Torre', M. Bonato ${ }^{1}$ and M. Gerzevic ${ }^{2}$ \\ ${ }^{1}$ Department of Biomedical Sciences for Health, Università di Milano, \\ Italy
}

${ }^{2}$ Institute of Kinesiology Research, Science and Research Centre of Koper, University of Primorska, Slovenia

Introduction: Physiological demands and responses to ultra-endurance (UE) cycling are still need to be evaluated despite the increased number of participants. The purpose of this study was to examine the physiological demands of an UE road cycling event across the Alps and its effects on blood inflammation markers and energy intake.

Method: One recreationally well trained man subject ( $26 \mathrm{yrs}, 80 \mathrm{~kg}, 187$ $\mathrm{cm}$, VO2max: $64.8 \mathrm{ml} / \mathrm{O} 2 / \mathrm{kg}, 315 \mathrm{~W}$ at $3 \mathrm{mmol} / \mathrm{L}$ of blood lactate) covered $692 \mathrm{~km}$ from Freiburg (D) to Koper (SLO) with difference in altitude of $2700 \mathrm{~m}$, in $28 \mathrm{~h} 23 \mathrm{~m}$ (temperature ranged between: $6^{\circ} \mathrm{C} /+20^{\circ} \mathrm{C}$ and $40 \%$ of night time). Heart rate (HR), power output (PO), food and beverage intake were recorded during cycling; six blood inflammation markers (C-Reactive Protein: CRP; Myoglobine: MB; Creatine Kinase: CK; Lactate Dehydrogenase: LDH; Leukocyties: LC; Creatinine: CRN) were measured 3 days before and immediately after UE effort.

Results: The subject cycled at low intensity (HR: $120.7 \mathrm{bpm}, 67 \%$ HRmax, PO: $177.7 \mathrm{~W}, 49 \%$ POmax and VO2max: $2360.43 \mathrm{l} / \mathrm{m}, 45 \%$ VO2max) $(1,2)$. The PO profile shows that the subject started to pedal over $60 \% \mathrm{VO} 2 \mathrm{max}$ and slowly the intensity decreased to $40 \% \mathrm{VO} 2 \mathrm{max}$ towards the end. The cyclist ingested $2430 \mathrm{kcal}$ more than it was planned $(6058 \mathrm{kcal})$ and he drank $9850 \mathrm{ml}$ with $2335 \mathrm{kcal}$ dissolved. Inflammation markers were elevated soon after riding (CRN: $+16.88 \%$; CRP: $+5514 \%$; LDH: $+68.97 \%$; CK: $+300.77 \%$; MB: $+446 \%$; LC: $+45.05 \%)$.

Discussion: As this case study the UE cycling is usually performed at low metabolic intensity as literature confirmed $(1,2)$, the $67 \%$ HRmax agrees with the theoretical approach of the UE threshold $(1,2)$. According with the cyclist strategy, considering the prolonged exercise, he started riding with maximum PO sustainable for every single moment, therefore this high stress conditions probably cause the decrease PO that could be due of the impairments of central nervous system and muscles compound. Despite nutritional status is well controlled the limit could be also attribute to the energy supply. As postulated by the psychobiological model based on motivational intensity theory (3) we cannot entirely explain the drop in PO, and the motivational level should be also take into consideration to success in UE sports. Furthermore, the high release of blood and muscles inflammation markers along UE cycling exercise could limit the performance in order to pr! eserve the human being. Concluding, the PO values confirms the exercise tolerance fall (3) and the fatigue raised. In order to success in UE, we suggest coaches to prepare the athletes to adapt themselves to prolonged exercise underling the motivational training (3). 1- Neumayr G. 2004 doi: 10.1136/bjsm.2002.003707. 2- Neumayr G. 2003 doi: 10.1136/bjsm.37.1.89 3-Marcora 2010 EJAP doi 10.1007/s00421-0101418-6.

Key words: cycling, ultra-endurance, heart rate, power output, blood inflammatory markers, food intake and beverage intake.

\section{Tactical comparison in women waterpolo through video analysis}

\section{S. Napolitano ${ }^{\text {, P.A Di Tore }}$, G. Raiola ${ }^{I}$ \\ ${ }^{I}$ Facoltà di Sceinze della Formazione, University of Salerno, Italy}

The present work concerns the tactical evaluation in water polo and is a natural extension of a previous (study presented at ECSS 2012) that showed a significant correlation between the compliance of attack patterns implemented during game with attack patterns designed during training and the final result of the action. 
The analysis was performed on 9 women A1 division matches played by the Volturno S.C.

In these matches were analyzed the tactical schemes, on offense and defense, realized by different teams. For each team, different tactical situations have been identified ( under equal conditions, numerical inferiority or numerical superiority). Aim of this work is to analyze the relationship between the coefficients of offensive and defensive effectiveness and compliance of patterns implemented during game with patterns designed during training.

This study is thus a pilot work; adopted method involves the quantification of tactical variables and the definition of offensive and defensive effectiveness coefficients, absolute and relative, through the adoption of a control board validated in previous studies ((Argudo, F \&. Lloret, M. 1999). By way of explanation, here are reported criteria for the calculation of several coefficients taken into consideration:

Effectiveness Coefficient $=$ success actions number $/$ actions total number;

Game level coefficient $=$ actual playing time $/$ (number of points in favor - number of points against)

The data recruitment has been entrusted to the performance analysis, conducted by a team of performance analysts, coaches and statisticians. The video analysis of matches was conducted using the Dartfish TeamPro software.

"The practical value of performance analysis is that well-chosen performance indicators highlight good and bad techniques or team performances" (Hughes, Bartlett 2002)

The expected result is the determination of a significant correlation between the tactical patterns compliance and offensive effectiveness coefficients, in each of the examined conditions.

The existence of such correlation would confirm the need of developing a common methodology for teaching and training waterpolo through tactics.

\section{References}

- Argudo F, Lloret M. (1999) .Coeficientes de eficacia ofensiva y defensiva en Waterpolo femenino. Comunicaciones Técnicas. 2:45-6

- Napolitano, S., Di Tore, P. A., Raiola, G. (2012). Tactical analysis on 9 women water polo matches during The 2011-2012 Regular Season. Paper presented at the 17th annual Congress of the European College of Sport Science

\section{Postural analysis screening on 14 women water polo players}

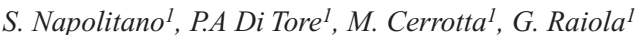

\section{${ }^{1}$ Facoltà di Scienze della Formazione, University of Salerno, Italy}

The present study examined the incidence of postural aspects on wellness status and performance of 14 women waterpolo players of Italian A1 Championship. The research consisted in the assessing the 3D detection of the surface of the trunk of the athletes in order to detect the state of their vertebral column to evaluate the incidence of posture in the athletic performance. (Téczely \& ANGYAN, 2006).

Object of investigation, in particular, was the ability of the athletes body to produce adjustments capable of compensating the presence of pathological curves.

Screening was performed at Centro CORPORA of Gricignano (CE) with the "Spinometria Formetric" device, under agreements between the Centro CORPORA (which has provided the equipment) and the company Volturno SC (which has provided the athletes).

The "Spinometria Formetric" allows for quick detection of the 3D morphology of the trunk, with extreme accuracy (with a margin of error of less than $0.2 \mathrm{~mm}$ ), fast (a few minutes for the entire procedure) and safety (do not use ionizing radiation as the conventional radiography). The Formetric check-up provides a series of indicators which allow to obtain a detailed assessment of the subject posture, thus allowing complete clinical examination with quantitative elements.

Data collected show that pathological curves observed in athletes may be compensated by other adjustments of the body, produced in vigorous in water exercise and have no effect on the state of wellness nor on the performance, where states of soreness resulting in a lower performance levels have been detected in subjects who had no pathological curves.

- (Téczely, T. , Ángyán, L., Investigation Of Postural Stability, Reaction Time And Body Measures In Basketball Players, 2006)

\section{Rugby Sevens: a scientific road map to Rio 2016}

\section{G. Granatelli ${ }^{1}$, B. Ruscello ${ }^{I}$, S. D'Ottavio ${ }^{I}$}

${ }^{1}$ School of Sport and Exercise Sciences, Faculty of Medicine and Surgery, University of Rome Tor Vergata, Rome, Italy

The aims of our study were to investigate the process of fatigue in Rugby Sevens over time through the observed temporal patterns of some physical (speed zones) and physiological (Heart Rates) features, by the means of an analysis carried out on the whole of the games and minute per minute. We also tried to verify the difference of these parameters in relation to the positional play, according to the roles: back and forward. We think that the sevens presents a peculiar set of characteristics in consideration to the space, the play time and the number of players and so it is desirable to analyse it having a different point of view from the one usually utilized in other team sports. Nine Italian elite players men (age $25.1 \pm 3.1$ years; body mass $86 \pm 9.4 \mathrm{~kg}$; height 180.5 $\pm 3.5 \mathrm{~cm}$; BMI $=27.71 \pm 2.62 \mathrm{~kg} \cdot \mathrm{m}-2$; maximal oxygen uptake (VO2max) $52.1 \pm 3.4 \mathrm{ml} \cdot \mathrm{kg}-1 \mathrm{~min}-1 ; 5$ years of Sevens specific training), were monitored during 15 matches $\mathrm{a}$ ! t the "Rome Sevens" international tournaments, held in Rome in 2010 and 2011 through the GPS (SPI Elite, GPS Sports Systems Ltd., Camberra, Australia), 5 cardio-frequency meters (Team Polar, Polar Electro Oy, Kempele, Finland) and the software GPSport Team AMS v1.2.1.0. Percentage of time spent in each speed zones per minute, Repeated measure Anova: [Main Factor (MF): minute; Interaction (I): minute x role] $(0-6.0 \mathrm{~km} / \mathrm{h})(\mathrm{MF}): \mathrm{P}=0.017$, (I): $\mathrm{P}=0.21 ;(6.1-12.0 \mathrm{~km} / \mathrm{h}) \mathrm{P}=0.014, \mathrm{P}=0.017 ;(12.1-14.0 \mathrm{~km} / \mathrm{h}) \mathrm{P}=0.070$, $\mathrm{P}=0.461 ;(14.1-18.0 \mathrm{~km} / \mathrm{h}) \mathrm{P}=0.070, \mathrm{P}=0.120 ;(18.1-20.0 \mathrm{~km} / \mathrm{h})$ $\mathrm{P}=0.704, \mathrm{P}=0.581 ;($ Over $20 \mathrm{~km} / \mathrm{h}) \mathrm{P}=0.632, \mathrm{P}=0.507$. Peak (HR per$\mathrm{min}$ ) during the matches: mean absolute values recorded per minute in all the matches: [(min. 1) $157.30 \pm 22.90)$, (2) $177.10 \pm 10.71$, (3) $182.00 \pm 9.60$, (4) $184.20 \pm 8.42$, (5) $178.20 \pm 13.44$, (6) $182.00 \pm 10.40$, (7) $181.20 \pm 7.56$, (8) $164.50 \pm 14.81$, (9) $175.10 \pm 8.38$, (10) $180.80 \pm 7.22$, (! 11) $181.80 \pm 10.05$, (12) $182.80 \pm 15.59$, (13) $181.20 \pm 17.17$, (14) $178.80 \pm 16.61]$. Having found a certain consistency with some pattern of behavior, both from a physiological and a kinematics point of view, we might say that a match of sevens, though divided in two halves by the regulation, may actually be considered as a single performance, continued for 14 minutes, where quite often the dynamics we found might be labeled as follows: (Phase 1) (1st-2nd minute) intense starting; (2) (3rd4th minute) maintaining phase; (3) (6th-7th minute) a crescendo final; (4) (8th-10th minute) recovery of the initial levels and progressive increase; (5) (11th-14th minute) significant decrease. Match management: 1) Adeguate warm-up Adequate arousal Clear strategic plans; 2) implementation of the strategic plans and tactical adaptation; 3) maximum effort to take the advantage and to control the pace of the game (take the lead); 4) readjustment and implementation of the strategic plans at high speed; 5) Fatigue! Management. Match Economy: controlling the risk rate. 
Correlation between ballistic stretching and conditional capacity: explosive strength training in young athletes practicing karate

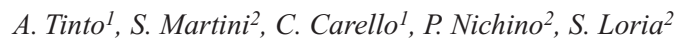

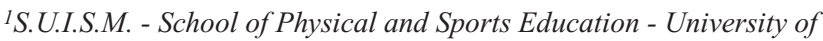 \\ Turin, Italy \\ ${ }^{2}$ Italian Karate Federation
}

Aim: The purpose of this study is to find a method for explosive strength training in the pre-puberty and puberty ages, as they are periods, in which bones, muscles, tendons, ligaments and cartilages are very sensitive to overloading and, therefore, risk being damaged easily in case they stressed by plyometric training.

Methods: The study was conducted on a group of pre-agonist karate athletes with ages between eight and twelve years (age 10,5 \pm 2.2 years, weight $44.34 \mathrm{Kg} \pm 4.5$, height $138.94 \pm 5.6 \mathrm{~cm}$ ) featuring both genders heterogeneously, which was divided into the experimental group (EG) and the control group (CG). The experimental group was administered regularly with joint mobility protocols that focused on the use of ballistic stretching exercises. An initial protocol was maintained for two months, followed a one-month break, and, subsequently, a new protocol, similar to the first one by content and methods was administered on the GL. The control group pursued standard training protocols. The experiment lasted a year. Three assessments were conducted: at the start, middle and end of the project.

Results: The tests which were carried out on the athletes at the beginning and end of the experiment focused both on the explosive strength and joint mobility: long jump from standing, triple jump, bending forward of torso, jumps on the spot with even feet ( 4 jumps). The EG showed a statistically significant improvement in $75 \%$ of the tests, than the CG, according to the $\mathrm{P}$ value for Wilcoxon test and Mann-Whitney test. In the final comparison, the difference between the groups is significant for the test 1 (long jump, $\mathrm{P}=0.0180$ ), for the test 2 (triple jump, $\mathrm{P}=0.0034$ ), for the test 3 (Sit \& Reach $=0.0053$ ), for test $4(\mathrm{SJ}, \mathrm{P}=0.0094)$ and for the test $5(\mathrm{CMJ}, \mathrm{P}=0.0170)$ while there are no significant differences in reactivity test and in test 2 of flexibility.

Conclusions: The decline in results observed between $\mathrm{T} 0$ and $\mathrm{T} 1$ may be attributed to the difficulty encountered by the athletes when adding some new exercises to their training programs and the need to perform them with utmost precision. The purpose of the constant presence of observers during training, especially at the beginning, was to help coaches by correcting the execution procedures, if necessary, and to explain the importance of certain principles in the execution of the exercises.

The high percentage of improvement obtained between $\mathrm{T} 0$ and $\mathrm{T} 3$ indicates, apart from the results that were statistically proven, the seriousness of the athletes during a part of training, which was not always appreciated and often repetitive.

\section{Experimental study: plyometric training in Rhythmic Gymnastic}

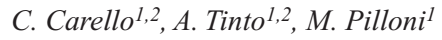 \\ IS.U.I.S.M. - School of Physical and Sports Education - University \\ of Turin, Italy \\ ${ }^{2}$ Italian Gymnastics Federation
}

Aim: The aim of this study was to evaluate the effects of plyometric training on a group of athletes who practice rhythmic gymnastics.

Subjects: The group consisting of 17 female gymnasts, with ages between 14 and 22 years, was divided into two subgroups: the first group, consisting of subjects with ages between 14 and 17 years (age 15.2 \pm 1.1 yrs; weight $47.3 \pm 4.9 \mathrm{~kg}$; height $162 \pm 4.4 \mathrm{~cm}$ ); and the second group, consisting of subjects with ages between 18 and 22 years (age 19.6 \pm 1.2 yrs; weight $54.6 \pm 3.9 \mathrm{~kg}$; height $166.7 \pm 6.1 \mathrm{~cm}$ ).

All of the subjects performed the same exercise: the first period (October through January) was characterized by "regular" preparation, without the addition of specific plyometric exercises, and the second period (March through May) featured the addition of an experimental protocol of specific plyometric exercises.

Methods: The duration of the motor project was 8 months with three workout sessions for week. The last part of each session was devoted to the experimental part that characterized the specific physical preparation of gymnastic jumps. Three evaluations were made: initial, intermediate and final assessments. The following tests were proposed, which were carried out using Optojump: squat jump, stiffness, gymnastic jump, squat, standing calf exercises.

The following exercises were proposed during the experimental protocol: drop jump ( 7 repetitions for 5 series with a drop from a height of 50 $\mathrm{cm}$; 14 repetitions for 5 series with a drop from a height $20 \mathrm{~cm}$ ) and step-climbing $(50 \mathrm{~cm}) 7$ repetitions for 5 series.

Results: According to the results, there is a slight improvement in both group 1 and group 2. Group 2 showed more evident improvements. As a matter of fact, their improvement in the squat jump test was $0.4 \mathrm{~cm}$; in gymnastic jumping, the improvement was $1.5 \mathrm{~cm}$ and in stiffness test, an improvement of $1.1 \mathrm{~cm}$ was recorded. The flight time deteriorated by 0.02 seconds, the contact time decreased by 0.07 seconds (improved responsiveness). Muscle power increased by 6 repetitions during squat jump and 7 repetitions during the execution of the calf exercises.

The statistical analysis performed (non-parametric Wilcoxon test) showed the validity of the experimental method with significant differences between the two work methods for some tests only.

Conclusions: According to the results obtained, muscular variables and responsiveness have positive impact on the final execution of the jump. Since the two groups were of different ages, further study is required to investigate the selection, repetitions and the procedure of performing the exercises suitable to the gymnasts' ages and the competitive level practiced.

\section{Biomechanical aspects of running analysis: technical shoes vs barefoot conditions}

\section{Russo, G. Petrollini, M. Mondonico, E. Guerra}

\section{ELAV Institute, Città di Castello, Italy}

Introduction: Barefoot running is becoming a trend, it allows an higher stride frequency, a lower stride length, a lower ground reaction force and a lower foot pronation in the loading phase respect to the shod running exercise (Divert 2005, Bishop 2006; Divert 2008; Squadrone 2009; Jungers 2010; Lieberman 2010). Literature took in consideration trained and expert people. Few data are available about novice to barefoot conditions. Aim of this study is to compare the acute kinematic and dynamic effects of barefoot running in novice people.

Methods: 13 healthy and physically active men $(28.7 \pm 5.2$ y.o., $173.8 \pm 3$ $\mathrm{cm}, 70.7 \pm 4.8 \mathrm{~kg}$ ) were tested. The participants were volunteers and each one was informed about the study procedures and gave his consent for the tests. The subjects were tested in three different conditions: using an anti-skid sock (SOCK), using Revolution (Akkua, Brescia, Italy) a special lightweight shoe (REV) and using their own running shoes (SHOE); condition sequence was random assigned for each trial on the treadmill. Testing session lasted two days: day 1 subjects ran at 5 and $11 \mathrm{~km} / \mathrm{h}$, day 2 subjects ran at 8 and $14 \mathrm{~km} / \mathrm{h}$. Each test lasted 6 minutes, work $/$ rest ratio was 1:1. During each test the subjects were video recorded at $240 \mathrm{fps}$ (Casio Exilim FH-100, Shibuva, Tokyo, Japan) and analyzed with an inertial sensor at $200 \mathrm{~Hz}$ applied in the lumbar zone (Sensorize Free Sense, Roma Italy). Sole angle, ankle angle at impact, stride frequency and 
length, peak acceleration, vertical displaceme! nt were measured. Kolmogorov-Smirnov, and ANOVA for repeated measures were used for statistical analysis.

Results: No significant differences in any parameter were measured between SOCK and REV. SHOE was significant higher in sole angle respect SOCK and REV for each speed. The opposite happened for the ankle angle at impact. No significant difference between the three conditions was present for the other parameters.

Discussion: Barefoot running in novice subjects does not significantly change frequency and length of the stride but changes the sole angle and the approach of the runners' feet with the ground. We think that a training period in barefoot condition is necessary to avoid injuries, cause the absence of difference in peak acceleration between barefoot and shod running. The special lightweight shoe Revolution is identical to the real barefoot condition (SOCK): it is useful to barefoot running offering a correct and essential protection of the foot.

\section{References}

1. Bishop M. et al. (2006) J Athl Train 41(4):387-392

2. Divert C. et al. (2005) Int J Sports Med 26(7):593-598

3. Divert C. et al. (2008) Int J Sports Med 29(6):512-518

4. Junger WL. (2010) Nature 463(7280):433-434

5. Lieberman DE. et al. (2010) Nature 463(7280):531-535

6. Squadrone R. (2009) J Sports Med Phys Fitness 49(1):6-13

\section{"Sellaronda ski-marathon": exercise intensity and physiological demands} A. Savoldelli, A. Brighenti ${ }^{1}$, R. Modena ${ }^{1}$, B. Pellegrini
L.,

${ }^{1}$ CeRiSM, Research Center Sport Mountain \& Health, University of Verona, Rovereto, Trento, Italy

${ }^{2}$ Department of Neurological, Neuropsychological Morphological \& Movement Science, University of Verona, Italy

Introduction: Exercise intensity and physiological demands were measured during endurance competitions such as running, XC skiing and offroad cycling while we have found only one study which analyses a short $(15 \mathrm{~km}) \mathrm{WC}$ ski-mountaineering race. The aim of this study was to quantify the exercise intensity of a particularly long ski mountaineering race, the Sellaronda skimarathon $(42 \mathrm{~km} ; 2700 \mathrm{~m}+$ divided in four different uphills).

Methods: Ten trained athletes (VO2max 61.9 \pm 3.2 mlO2*kg- $1 *$ min- 1 ), who participates to the Sellaronda ski-marathon, performed a graded exercise test to exhaustion with roller skis adapted for ski-mountaineering on a treadmill in order to evaluate the first ventilatory threshold (VT1) and the respiratory compensation threshold (RCT). Three exercise intensity zones were determined (Z1:less VT1, Z2:between VT1 and RCT, Z3: above RCT). During the competition heart rate (HR) was continuously recorded and then evaluated respect the different zones. Energy cost (EC) during the ascents was calculated from the HR-VO2 relationship obtained in the maximal test.

Results: VT1 and RCT thresholds were found at $84.4 \pm 1.8 \%$ and $94.0 \pm 1.0 \%$ of HRmax and at $73.7 \pm 4.1$ and $89.4 \pm 2.9 \%$ of VO2max. The mean race time was $267 \pm 43$ minutes $(82.7 \%$ of which spent during ascents) and the mean HR was $84.6 \pm 2 \%$ of HRmax. The percentages of race time spent in $Z 1, Z 2$ and $Z 3$ were $41.4 \pm 13.0,57.5 \pm 12.3,1.0 \pm 2.4 \%$, respectively. The mean value of EC during the four uphill of this race was $10.86 \pm 1.35 \mathrm{~J} * \mathrm{~kg}-1 * \mathrm{~m}-1$. A significant correlation $(\mathrm{R}=-0.88, \mathrm{P}=0.009)$ has been found between time spent to climb the 4 uphills and the percentage of HR at RCT. The correlation between the parameters obtained during the incremental test and the total race time is not significant. We found a significant decrease of $\%$ of HRmax and $\%$ of HR at RCT across the 4 ascents. However there is a significant increase of the climbing speed during the 4 uphills and an increase of the slope $(8.7 \%, 11.9 \%$, $13.3 \%, 18.7 \%$ ).

Discussion: Our results demonstrates that in this kind of competition the majority of race time is spent in moderate and low intensity zones. Moreover the significant correlation between time spent to cover uphill tracts of the competition and the \% $\mathrm{HR}$ of RCT bring us to sustain that great aerobics capacities are needed for achieve good results in skimarathon races. The mean HR\% found in this study and the time spent in $\mathrm{Z} 3$ zone are lower than those found for shorter races, and confirms the knowledge that HR decreases with exercise time.

References:

- Achten, J. and A. E. Jeukendrup (2003). "Heart rate monitoring: applications and limitations." Sports Medicine 33(7): 517-538.

- Duc, S., et al. (2011) "Physiology of Ski Mountaineering Racing." International journal of sports medicine. 32:856-863

- Tosi, P., et al. (2009). "The energy cost of ski mountaineering: effects of speed and ankle loading." Journal of sports medicine and physical fitness 49(1): 25-29.

\section{Overload and neovascularization of shoulder tendons in volleyball} players

\section{A. Notarnicolal, ${ }^{1,2}$,F. Fischetti ${ }^{1}$, D. Gallone ${ }^{1}$, L. Moretti ${ }^{2}$, S. Tafuri ${ }^{3}$,} B. Moretti $^{1,2}$

${ }^{1}$ Corso di Laurea in Scienze delle Attività Motorie e Sportive, Università di Bari, Italy

${ }^{2}$ Dipartimento di Neuroscienze ed Organi di Senso, Sezione di Ortopedia, Università di Bari, Italy

${ }^{3}$ Dipartimento di Scienze Biomediche, Sezione di Igiene, Università di Bari, Italy

Background: In overhead sports like volleyball, the onset of a rotator cuff tendinopathy due to functional overload is a common observation. An angiofibroblastic etiopathogenesis has been hypothesized, whereby a greater anaerobic metabolism occurs in critical zones of the tendon with a lower degree of vascularization; this would induce collagen and extracellular matrix degradation, that could then trigger a compensatory neovascularization response.

We performed a clinical observational study of 80 elite volleyball players, monitoring the perfusion values of the supraspinatus tendons by oximetry.

Results: No statistically significant differences were found between the oximetry data and age, sex or years of sports activity, nor when comparing the right and left arm or the dominant and nondominant arm. A statistically significant difference was found for the dominant arm values in relation to the competitive role, higher values being obtained in outside hitters $(62.7 \%)$ than middle hitters $(53.7 \%)(p=0.01)$, opposite hitters $(55.5 \%)(\mathrm{p}=0.02)$ and libero players $(54.4 \%)(\mathrm{p}=0.008)$, whereas there were no differences in setters $(56.2 \%)(\mathrm{p}>0.05)$.

Conclusions: The different tendon vascularization values found in players with different roles in the team may be attributed to a response to the specific biomechanical demands posed by the different overhead throwing roles. 


\section{ORAL PRESENTATIONS}

\section{Saturday, October $6^{\text {th }}, 2012$}

\section{Session 5.2. Sport Technology and Materials}

\author{
The Repeatability of Jump tests measured with the Opto Jump
}

M. Golllin, E. Scarafiotti

Motor Science Research Center, University School of Exercise \& Sport Sciences, University of Turin, Italy

Aim: Repeatability evaluation of the flight time (FT) tests for SJ, CMJ,
CMJBL and SPJ (Spike jump)
Methods: Tests were carried out on 8 volleyball players (age $16 \pm 1$, height
$166,4 \pm 8 \mathrm{~cm}$, weight $65 \pm 8 \mathrm{~kg}$ ) who had been trained at least twice a
week and for no fewer than three years. Before beginning the repeata-
bility protocol, the participants had completed one month of training in
the jumping techniques in order to reduce the learning effect. The pro-
tocol consisted of 3 tests per day on 3 different days; each test day was
followed by a day of rest. The recovery time between each test on a giv-
en day was 60 minutes. After a specific warm-up, the athletes moved on-
to the tests which consisted of 3 trials for each type of jump with a re-
covery time of 60 seconds, and of three minutes between different jump
types. Statistical Analysis: The ICC, the variance between the tests and the variance between the days were calculated. Furthermore, the standard error of the mean was calculated within (SEMw) and between (SEMb) the participants.

Results: The data shows that the opto jump allows a repeatable measurement of flight time (FT) with an average ICC of $95 \%$. The percentage of mean variance due to the tests $(4 \%)$ was found to be greater with respect to the mean variance between days $(2 \%)$. The SEMw was $1 \%$, while the $\mathrm{SEMb}$ was $3 \%$.

Discussion/Conclusion: The FTs in each test, with ICC value, were greater than $90 \%$. Furthermore the results confirm not only the high level of repeatability of the measurements but also that the observed differences were mainly due to individual differences between the participants.

Stabilometric and electroencephalographic monitoring of sea wave- motion effects during a sealing boat trip

\section{Pizzigalli, D. Formicola, M. Ivaldi, A. Rainoldi}

Centro Ricerche Scienze Motorie, Scuola Universitaria Interfacoltà in Scienze Motorie, Università degli Studi di Torino, Italy

To maintain balance, information from visual, vestibular and proprioceptive sensory systems are required. When one or more inputs is distorted or absent, the CNS is able to manage the situation adapting the input/output ratio. As already known, exposure to sea condition results in both intravestibular and vestibular-visual conflicts. This work aims to analyze the adaptation of the postural sensory systems during a sailing boat trip in male and female subjects.

Fifteen young subjects ( 11 males and 5 females; average age $20 \pm 2$ years; weight $74 \pm 8 \mathrm{~kg}$; height $177 \pm 9 \mathrm{~cm}$ ) performed balance tests on a posturographic balance platform (ProKin, TecnoBody, Bergamo, Italy) in bipodalic stance position (60 s), with (EO) and without (EC) visual inputs before, during and after a sealing boat trip. In nine participants EEG signals were recorded, using a wireless device (OtBioelettronica, Torino, Italy) during bipodalic tests in EO and EC conditions. Mean frequency and mean amplitude from the total scalp were calculated from signals recorded. According to the sensory rearrangement theory, center of pressure (COP) data monitored during three days of navigation will show the subject adaptation to this unusual moving environment. During quay trials (embarkation) and during the sailing boat trip at stern, no statistical differences where found between male and female groups (U Mann-Whitney test). On the contrary, during the sailing ! boat trip, trials at prow showed statistical significant differences between the two groups $(\mathrm{p} \leq 0.03)$. Both in EO than in EC condition female group reported the best balance performance for mean velocity in anterior-posterior and in medium-lateral plane, perimeter length and ellipse area of the COP. During quay trials (debarkation) male showed worse postural stability than female group for COP area $(p<0.05)$ in both visual conditions. However, an increase of balance performance was observed in male group between the first and the latest trial at prow position both in EO and in EC condition (Wilcoxon test $\mathrm{p} \leq 0.005$ ). Such findings will be related with results obtained from EEG signals. Data collected, for the first time during a sealing boat trip, supported the existence of gender based differences in postural ability both in a motion environment (navigation) and also in a static situation after a one week conditioning (debarkation).

aGrisù: a patent project to highlight how physical activities affect the rate of absorption of air pollutants

\author{
M. Ivaldi ${ }^{1}$, M. Turturici ${ }^{2}$, L. Ferreri ${ }^{3}$, L. Feletti $^{1}$, M. Giacobini ${ }^{3}$, \\ A. Rainoldi ${ }^{I}$
}

${ }^{1}$ Centro Ricerche Scienze Motorie, SUISM, Università degli Studi di Torino, Italia

${ }^{2}$ Dipartimento di Neuroscienze, Università degli Studi di Torino, Italia ${ }^{3}$ Gruppo di Epidemiologia Computazionale, Dipartimento di Scienze Veterinarrie, Università degli Studi di Torino - Unità di Sistemi Complessi, Centro di Biotecnologia Molecolare, Università degli Studi di Torino, Italia

Aim: A number of papers in literature show that physical activities increase the exposure to air pollution and the poisonous deposition within the body. In fact, physical activities increase pulmonary ventilation and hence the volume of inhaled gases, harmful or not. A device providing the level of various air pollutants with respect to own physiological parameters was designed and implemented to became a tool for individual health awareness relating air quality according to exercise performance. Methods: The general architecture of $a$ Grisù is made by:

-a wearable sensor for the detection of physiological parameters (heart and respiratory rate), communicating via BT technology with a mobile terminal;

-a mobile terminal equipped with BT, GPS, GSM technology, communicating to a Main Server physiological parameters, received from the wearable sensor, and the position, detected by GPS;

-monitoring stations for the detection of air pollutants: Carbon Monoxide (CO), Sulfur dioxide (SO2), Metals (Lead, Arsenic, Cadmium, Nickel), Benzene $\left(\mathrm{C}_{6} \mathrm{H}_{6}\right)$, Nitrogen Dioxide $\left(\mathrm{NO}_{2}\right)$, Ozone $\left(\mathrm{O}_{3}\right)$ and Particulate matter $\left(\mathrm{PM}_{10}, \mathrm{PM}_{5}, \mathrm{PM}_{2,5}\right)$;

-a Main Server bringing together morphological and physiological parameters, geographical and environmental data (derived from the public servers and specific monitoring locations). The Main Server processes the data obtained and sends to the mobile terminal customized information about air quality and its influence on the physical health.

It's not possible to specify in details the structure of the algorithm because the patent pending.

Conclusions: For our knowledge $a$ Grisù is the first system that try to 
provide a real-time indication of how physical activities affect the rate of absorption of air pollutants. $a$ Grisù could offer a more conscious vision about consequences of practising physical activities in certain area. Moreover, $a$ Grisù could raise awareness about air quality, driving a change in the policies for health protection.

\section{Analysis of skinfold thickness: a comparison among skinfold caliper, ultrasound scanner and ultrasound based caliper}

\section{Formicola, M. Ivaldi, A. Rainoldi}

Centro Ricerche Scienze Motorie, SUISM, Università degli Studi di Torino, Italia

Aims: The importance of a correct body composition for a healthy and long-term life has been confirmed by scientific literature in the last decades. In particular, an excess of body weight is one of the the major risks of all-cause of mortality and cancer. Therefore the importance of an accurate and precise measurement is evident.

The study aims to compare three different instruments used to measure skinfold thickness: skinfold caliper, ultrasound scanner and BodyMetrix (an ultrasound based caliper with head@2.5 MHz) and to investigate repeatability and reliability of estimates.

Methods: The study was conducted analyzing ten subjects (five male and five female). For each of them we measured 19 skinfolds for each side; any skinfold was measured in three places (medially, proximally and distally with a distance of $2.5 \mathrm{~cm}$ in between them) in four different times (after three hours from the first measurement, after three and after nine days) for a total of 13,680 observations. The statistical analysis aimed to assess reliability and repeatability of the three devices describing thickness estimates as a function of the sources of variability (location, body area, subjects). A suited Matlab interactive routine was created to allow the analysis of ultrasound images.

Results: Non parametric statistic procedures were executed to compare the three devices (Dunn-Sidak Post Hoc Test after Kruskall Wallis with critical value fixed at 0.05$)$. Statistical significant differences $(p<0,05)$ were found in ultrasound machine between distal and proximal positions. These differences were highlighted in male. Comparisons among the three devices pointed out significant differences in skinfold thickness estimates in the following anatomical sites: bicipital, tricipital, chest, subscapular, costal, suprailiac, abdominal, lumbar and calf. Only ultrasound scanner was able to discriminate small displacements with respect to the median skinfold. In no cases was highlighted a significant change in skinfold thickness during the nine days of observation. Finally, no differences were observed among the three devices in male or female estimates and in right or left side estimates.

Conclusion: They offer quite comparable repeatability and standard error of the mean. They are non invasive but ultrasound machine was found to be more accurate in highlight minor displacement but it is the most expansive among the three devices. Bodymetrix is an accurate instrumentation, with good reliability and repeatability, but it doesn't do a substantial improvement of accuracy of measurement, in fact BodyMetrix has less accuracy than other instruments $(\sigma$ BodyMetrix $=$ 10,$50 ; \quad \sigma_{\text {Caliper }}=8,82 ; \quad \sigma$ Ultrasound 7,59 ) the caliper is, as known, the most cheaper and easy to use.

Postural Interventions as instruments to improve the physical wellness: a research-intervention in a sicilian soccer school

\section{Francavilla, F. Sgrò, P. Mango, S. Pignato, G. La Delfa}

Engineering, Architecture and Physical Activities Faculty, University of Enna, Italy
Introduction: The control of the posture represents a function of the utmost importance which allows the movement optimization and make it more functional minimizing its energy expenditure.

The Tonic Postural System manage this process, through a set of receptors, nerve centers, and control effectors which are capable of correcting any variation of equilibrium conditions. Complex biomechanical rules expressed in three moments: the posture in physiological values achieved in static, dynamic, and cybernetic conditions.

The latest is also a psychological variable which allows a relationship between human and their environment through the "body language". The human body favors the lowest energy cost, which enables it to communicate with the external environment by means of a system extraverbal. Methods: In this work we addressed a randomized sample of 300 athletes, aged between 8 and 16 years practicing soccer, at the same school, with the same frequency during the year. We provided a past medical history, proximate and remote, for all of the participants. Furthermore, athletes were observed through a clinical global postural examination, baropodometric, stabilometric, and posturographic tests.

Results: Our data revealed an alarming trend: $90 \%$ of the sample children presented a postural deficit, $75 \%$ of whom complained of pain, and $50 \%$ reported recurrent injuries that forced the dropout of the training camp.

The most shocking data were:

-) The excessive use of NSAIDs in young;

-) The $95 \%$ of patients having postural deficits wore everyday shoes with anti-physiological characteristics.

Discussion: In the context of the epidemiological study presented, it was carried out a research on the usefulness of postural and proprioceptive gymnastics administered by the graduate in Sports Science combined with a normal physical training of the young soccer players and active Participants were divided into three groups, with well-coded inclusion criteria, and compared with each other both clinically and athletically. Findings outlined that the usefulness of the gymnastics and active proprioceptive plantars, with both morpho-functional and postural improvement.

Furthermore, these solutions can improve children performance and their wish to play sports following.

\section{Contribution to the definition of the performance model of triathlon using Notational analysis}

\section{P. Mango, F. Sgrò, S. Di Martino, S. Pignato, M. Lipoma}

\section{Engineering, Architecture and Physical Activities Faculty,} University of Enna, Italy

Introduction: In recent years, there has been a growing interest among people in ultra-endurance events, such as the Ironman triathlon, although there are only a few scientific studies about this sport. The study was carried during the latest Deca Ironman European Championship, held in Enna (Italy).

19 athletes (17 males and 2 females), from different countries, were involved in the international competition and were asked to participate in our research. All participants signed an informed consent prior to data collection.

The Ironman triathlon race consists of $3.8 \mathrm{~km}$ of swimming, $180 \mathrm{Km}$ of cycling and $42 \mathrm{Km}$ of running, raced in that order and without a break. Deca Ironman is a ten Ironman-distance triathlons in 10 days, in which athletes swim for $38 \mathrm{Km}$, ride on bikes for $1800 \mathrm{Km}$ and run for $420 \mathrm{Km}$. Objective: The study aimed to define a triathlon performance model, throughout the analysis of athletes' performance during the race.

Method: Total times and single discipline times for each athlete were measured with chronometers (for swimming) and sensors (for cycling and running). Using a Notational Analysis Methodology, athletes were 
observed in their performance for ten days, both in performances and management of exertion.

Results and discussion: 12 athletes completed the race. The winner completed the race after 120 hours, the worst took approximately 200 hours to finish. Data provides a descriptive model of Deca Ironman athletes' performance. Further research should investigate the relationship between ultra-triathlon performers presented in this work and other variables, such as psychological aspects, nutrition, and physiological parameters.

\section{ORAL PRESENTATIONS}

Saturday, October $6^{\text {th }}, 2012$

\section{Session 5.3. Testing and Performance Evaluation}

\author{
Fractal dimension and sway ellipse area comparison for postural \\ stability assessment
}

\author{
F. Masedu ${ }^{1}$, R. Di Giminiani ${ }^{1}$, A. Di Giulio ${ }^{1}$, J. Tihanyi ${ }^{2}$, M. Valenti $^{1}$ \\ ${ }^{1}$ Department of Applied Clinical Sciences and Biotechnology, \\ University of L'Aquila, Italy \\ ${ }^{2}$ Faculty of Physical Education and Sport Science, Semmelweis \\ University, Budapest, Hungary
}

Introduction: The recent literature regarding the issue of postural stability assessment in subjects with muscular-skeletal disorders, or neuromuscular pathologies affecting their motor skills, has suggested, both for practical and theoretical reasons, the use of some fractal dimension as a good outcome measurement for overall postural status $(2,3)$.

Objective: This study considers the issue of analysing postural sway by comparing the use of a fractal dimension outcome (DBOX) with the usual sway ellipse area (SEA), calculated with the least squares method (1). Both of the response variables come from centre of pressure (COP) dynamics that are detected by means of a force platform.

Methods: The general definition used for the dimension of an object with a hypervolume (i.e., length, area, volume or fractal hypervolume) given by $\mathrm{V}$ is $\mathrm{DBOX}=(\log \mathrm{N}-\log \mathrm{V}) / \log$ ? $(1 / \delta)$, where $\mathrm{N}$ is the number of hypercubes of side length $\delta$ required to cover the object, so that rearranging the definition, we have the equation of a straight line, where the gradient of the line DBOX is the box-counting dimension of the object: $\log$ ? $N=D B O X \log$ ? $(1 / \delta)+\log$ ? $V$, which is the form used for calculations (4). The DBOX was calculated using the box-count $\mathrm{m}$-file running on Matlab (MathWorks, Inc.), written by Frederic Moisy (downloadable at http://www.fast.u-psud.fr/ moisy/ml).

A sample group of 24 male subjects was recruited. The postural stabilogram was recorded both with eyes open (EO) and eyes closed (EC) while standing upright. A matched-pair comparison of the sway ellipse area with the COP dynamics box counting dimension was performed. A ROC analysis of the outcome variables was performed. Then, a ROC comparison of the tests, using the area under the curve (AUC) index, was conducted.

Results: The comparison of paired groups showed a statistically significant difference between EO and EC status, according to the sway ellipse area and the fractal dimension $(\mathrm{p}<0.05)$. The intraclass correlation was 0.62 . The ROC analysis that was performed, which described the test performance in terms of AUC difference, was statistically significant $(p<0.05)$. The comparison of the AUCs showed an overall superior performance of the DBOX with respect to the SEA $(p<0.05)$.

Conclusions: This study, focusing on the specific situation of the eyes being opened or closed, showed a statistically significant better overall performance of the DBOX with respect to the SEA, suggesting possible improvements of clinical practise, as well as theoretical insights into the response patterns.

\section{References}

1. Sparto PJ, Redfern MS. Quantification of direction and magnitude of cyclical postural sway using ellipses. Biomedical Engineering: Applications, Basis, and Communications, 2001; 13: 213-217.

2. Pascolo PB, Marini A, Carniel L, Barazza F. Posture as a chaotic system and an application to the Parkinsons disease Chaos, Solitons and Fractals 2005; 24: 1343-1346. 
3. Blaszczyk JW, Klonowski W. Postural stability and fractal dynamics. Acta Neuro Exp 2001; 61: 105-112.

4. Addison PS. Fractals and chaos: an illustrated course. IOP Publishing Ltd, Bristol, UK, 1997: pp 31-34.

The 3 minutes OptoJump® Step Test (3M-OST): repeatability of a new protocol to measure physical functions in elderly

\section{Roppolo, D. Magistro, A. Mulasso, P. Brustio, M. E. Liubicich}

Motor Science Research Center - S.U.I.S.M. - University of Turin, Italy

Introduction: the step test is a useful and very common test to study physical functions/efficiency. Unfortunately the majority of test protocols reported in scientific literature were studied for the young - adult population, with the set of very high step and a imposed frequency of movement.

Objective: to propose a new protocol of step test specifically designed for the elderly population and to assess the repeatability of the 3M-OST during a specifically designed protocol.

Method: the innovative aspects of the 3M-OST are: (a) the height of the step, setting at $16 \mathrm{~cm}$, as a common step (ecological situation), (b) application time, 3 ' that represents the time spend by elderly to cover about four floor of stairs, (c) the freedom rate of climb/descent, (d) the use of OptoJump for the evaluation of flight/contact time. The repeatability test consisted in 3 trials a day for 3 non consecutive days; the recovery time between two trials was set in 90 minutes. The test protocol consisted in a maximal three minutes of 3M-OST. Participants were 11 subjects (4 men and 7 women). The repeatability of the 3M-OST was calculated with the Intraclass Correlation Coefficient (ICC), the variance due to trials (VT) and the variance due to days (VD). Moreover, the standard error of the mean for each subject (SEMw) and between all subjects $(\mathrm{SEMb})$ were calculated.

Results and discussion: The results show that the 3M-OST offers a very repeatable measure of since ICC was found greater than $98 \%$. The portion of variance due to day-to-day repetitions $(0,1 \%)$ was found lower than the variance due to trial-to-trial repetitions $(1,1 \%)$. The SEMw was $1,2 \%$ and the SEMb was $9,4 \%$.

Conclusion: The high value of ICC confirmed the great repeatability of the $3 \mathrm{M}-\mathrm{OST}$, highlighting that the differences observed in the test were mainly due to subjects differences. Finally, 3M-OST estimates were available with high precision since SEMw was find lower than $2 \%$.

\section{Assessment and comparison of the motor abilities of young people normal weight and overweight/obese with the Fitnessgram protocol}

\section{Colella, M. Morano}

Department of clinical and experimental medicine, University of Foggia, Italy

Introduction: Physical activity is beneficial to health of children and should be a normal part of their growth and development1; physical education and sport in schools is essential to apply the Guidelines2. In the Apulia region of young 11,13,15 years showed percentages of overweight respectively of $21 \%, 16.65 \%$ and $14.29 \%$ and obesity by $3.8 \%$, $3.7 \%, 2.2 \%$ with values almost double of males than females 3 . The aim of the study was evaluate and compare the levels of motor performance according to difference of group ( $\mathrm{Nw} v s \mathrm{Ow}-\mathrm{Ob}$ ), with different protocols, for a systematic monitoring of physical and motor development of young people.
Methods: Motor test have been proposed4,5 : standing long jump; 20-m speed; curl-up; $90^{\circ}$ push-up; pacer $20 \mathrm{~m}$ (progressive aerobic cardiovascular endurance run) to 230 boys and girls middle school ( girls $=106$; age: $\mathrm{M} \pm \mathrm{ds}=12.26 \pm 0.89$; boys $=124$; age: $\mathrm{M} \pm \mathrm{ds}=12.29 \pm 1.0$ ). The sample was divided into groups according to BMI: normal weight and overweight / obese according to cutt off Cole6 (girls: $\mathrm{Nw} \mathrm{M} \pm \mathrm{ds}=$ $18,30 \pm 1,85$; Ow-Obese $\mathrm{M} \pm \mathrm{ds}=24.82 \pm 2,90$; boys: $\mathrm{Nw} \mathrm{M} \pm \mathrm{ds}$ $18.66 \pm 1.66$; Ow-Obese $\mathrm{M} \pm \mathrm{ds}=24.16 \pm 2,58$ ).

Results: Apart from the descriptive statistics, it was carried out the ANOVA 2 BMI (group) analysis of the variance; the significativity index was set to $p<0,05$. In relation to the group, the girls show differences in the tests: standing long jump $(p=0,003) ; 20$ m speed $(p=0,002)$; curlup $(\mathrm{p}=0,01)$; pacer $20 \mathrm{~m}(\mathrm{p}=0,01)$; the boys in the test: standing long jump ( $\mathrm{p}=0,001) ; 20 \mathrm{~m}$ speed $(\mathrm{p}=0,000)$; curl-up $(\mathrm{p}=0,01) ; 90^{\circ}$ push-up $(\mathrm{p}=0,009)$; pacer $20 \mathrm{~m}(\mathrm{p}=0,02)$.

Conclusion: The study confirms that normal-weight students showed strength and speed of motor performance higher than students in overweight / obese7,8. The different motor performance in relation to BMI are determinants for the evolution of motor of young. The levels of development of motor abilities are indicators of physical fitness and health for children and young people9. The monitoring of motor performance is needed for surveillance of health conditions5. Recent studies have shown that physical activity interventions conducted in schools to improve physical fitness of young 10 .

\section{References}

1. Brambilla $P$ et al. (2010), Int J Obes , 35,16-28

2. Janssen \& LeBlanc (2010), IJBNPA, 7, 40

3. http://www.epicentro.iss.it/territorio/puglia.asp

4. Morrow JR et al. (2005), Champaign, IL: HK

5. Meredith MD \& Welk GJ. (2007), Fitnessgram /Activitygram, Champaign, IL: HK

6. Cole TJ et al. (2000), BMJ, 320:1240-3

7. Poulsen AA et al. (2011), IJPO, 6, e464-e471

8. D'Hondt $\mathrm{E}$ et al. (2011), APAQ, 26,21-37

9. Ortega FB et al. (2008), Int J Ob, 32, 1-11

10. Kriemler S et al. (2011), Br J Sports Med, 45,923-930

Effectiveness of exercise training in increasing ultrasound-mediated improvements in body composition preliminary results F. Lucertini ${ }^{1}$, M. Greco
, M. Rinaldi
A. Dederici

${ }^{1}$ Department of Biomolecular Sciences, Division of Exercise and Health Sciences, University of Urbino "Carlo Bo", Urbino (PU), Italy ${ }^{2}$ Studio dermatologico Dr. Greco, Modena (MO), Italy

Ultrasound treatment is commonly used in aesthetic medicine as a nonsurgical method to reduce localized fat deposits. Transdermal focused ultrasound waves cause adipocytes cell membrane destruction: this lead to the liberation of the cell content in the interstitial space which, in turn, may increase high-energy substrate availability in the bloodstream. Highenergy substrate utilization is augmented by both aerobic and resistance exercise: fat is the major fuel during low-intensity aerobic exercise whereas resistance training increases post-exercise high-energy substrate utilization. Although ultrasound treatment has been proved to be a safe and effective method in reducing localized fat deposits, the possible adjunctive effects of aerobic and resistance exercises has never been studied. The aim of this study is to compare the effectiveness of combining exercise training and multiple ultrasound treatments (EU group) versus either exercise (E group) or ultrasound (U group) interventions alone. Sixteen young female subjects were randomly assigned to the $\mathrm{EU}(\mathrm{n}=9$; 
$29.3 \pm 3.8 \mathrm{yr})$ and the $U(\mathrm{n}=7 ; 32 \pm 4.9 \mathrm{yr})$ groups, while the recruitment of the $\mathrm{E}$ group is still in progress. Intervention of the EU group consisted in 10 ultrasound treatments (1/wk) plus combined walking and resistance training ( $2 / \mathrm{wk} \times 10 \mathrm{wks})$. U group intervention was identical but restricted to the ultrasound treatments. Before ( $\mathrm{t} 0$ ) and after (t1) the intervention, subjects underwent the following measurements: height $(\mathrm{m})$, weight $(\mathrm{kg})$, body circumferences $(\mathrm{cm})$, skinfold thickness $(\mathrm{mm}), 6$ minute walk distance (6MWD) covered (m) and the number of steps taken (a step counter was used) during a 6-minute walk test. Measurements were used to estimate body mass index (BMI) and fat mass (FM), while the 6MWD and the step count were used to prescribe the walking speed (step•min-1) of aerobic training in the EU group. Once-a-week, subjects exercised no later than $24 \mathrm{hrs}$ after the ultrasound treatment. E group has not been recruited yet, thus preliminary results focus on single-group (t0 $v s \mathrm{t} 1$ in either EU or E group) and between-group (EU $v s$ $\mathrm{U}$ of $\mathrm{t} 1-\mathrm{t} 0$ differences) comparisons (Wilcoxon matched-pair test or Mann-Whitney U-test for single-group or between-group comparisons, respectively).

Single group results evidenced, at $\mathrm{t} 1$, significant reductions (values in \%) of BMI (EU -3.4; U -3.1), weight (EU -3.3; U -3), FM (EU -5.5; U -4.9), and hip (EU -3.6\%; U -2.1), umbilicus (EU -2.6; U -1.7), right thigh (EU -4.4; U -3.9), and left thigh (EU -3.8; U -3.3) circumferences, whereas $6 \mathrm{MWD}$ improvement was non-significant $(\mathrm{EU}+5.6$; $\mathrm{U} 0.8)$ in both groups.

Although between group comparisons revealed no statistical differences among body shape and composition, single group results clearly showed superior effects of the combined intervention. Furthermore, 6MWD which represents a reliable cardiorespiratory health index - as expected showed a significantly higher improvement in the EU group compared to the U group.

\section{BMI, total hours of physical activity (CPM) and testing of boys and girls from the metropolitan area of Cagliari}

\section{Frau, A. Loddo, G. Cao, P. Pillosu, F. Marcello}

\section{CONI Provincial Committee of Cagliari, Italy}

Objectives: Most pre-adolescents and adolescents are not sufficiently engaged in physical activity, and this condition is not good for their health. There is a need for a precise and objective assessment of the percentage of youth who follow the recommendations of physical activity for health. Assess the physical activities and their entity and distribution, and the responses of the subjects to the various tests of motor assessment is of fundamental interest to better understand the phenomena related to adolescent lifestyles and to develop effective intervention programs.

Methods: A sample of 236 people was involved in the study: 10 to 21 years (138 males and 98 females) from sports clubs and secondary schools located in Cagliari and its hinterland. The following variables were measured: BMI, hours of operation / year (CPM), and various tests to evaluate motor skills.

Results: The average level of motor practice is around 55 minutes daily (S. Dev Std 26.43). Boys are more active than girls: 62 minutes against 46. The average BMI is equal to 20.75 (3.22 Std. Dev); 20.33 for boys, 21.35 for girls.

Conclusions: 69 girls (about 2/3 of the female sample) stood below the level of MVPA recommended for health, in the range 5 to 18 (60 minutes per day), while 81 boys were (slightly over a half of the sample). Also the average MBI for girls was higher (21.35 vs 20.33) with a few sporadic alarming cases. The maximum result among girls was 38.60 , while among boys was 30.40 .
The Shintaido training effects on physical and psychological health of elderly

\author{
A. Mulasso ${ }^{1}$, M. Roppolo $^{1}$, D. Magistro ${ }^{1}$, A. Roggero ${ }^{2}$, P. Andreoli ${ }^{1}$, \\ M. E. Liubicich ${ }^{1}$ \\ ${ }^{1}$ Motor Science Research Center - S.U.I.S.M., University of Torino, \\ Italy \\ ${ }^{2}$ Department of Psychology, University of Torino, Italy
}

Introduction: the elderly population and the life expectancy in the Western world are constantly increasing. This social change can be seen as a challenge to the identification of protective factors for maintaining as long as possible a good level of physical and psychological functioning in old citizens. Physical activity is one of the major protective factors on the elderly health. In this area of research is growing the interest in new oriental disciplines, as Tai Chi or Shintaido, that combined physical and mental aspects.

Objective: to investigate the effects of Shintaido activity in a group of elderly citizens in terms of physical and psychological health.

Method: 40 elderly subjects living in Piemonte participated at the study. The group was randomly divided in an experimental (EG) and control group (CG), that at baseline were homogeneous. The EG performed 20 weeks (twice a week, one hour per session) of Shintaido training. We collected data before (T0) and after (T1) the intervention using the following validated instruments: the Chair Sit and Reach test for measuring the flexibility of lower limb and trunk; the Back Scratch test for the upper limb flexibility; the Short Form-36 for the quality of life and the Self-Efficacy Perception in Physical Activity (APEF) for the self efficacy.

Results and discussion: the introduction of the Shintaido caused a significant increase in the physical - psychological functions in the EG. Regarding the physical aspects, we found an increase of the lower limb/trunk flexibility $(p=0.001)$ and upper limb flexibility $(p=0.001)$. For the psychological variables, we observed an improvement in quality of life $(p=0.001)$ and self-efficacy $(p=0.004)$. No differences were found for the CG.

Conclusion: the results highlight that Shintaido discipline can have a strong impact in physical and psychological health necessary to the maintenance of autonomy in activity of daily living in the elderly population. Further studies are necessary to prove the real effectiveness of the Shintaido training in a larger sample size and compared with most traditional training protocols.

\section{Whole body vibration training: a functional procedure for weight management}

M. Lombardo ${ }^{1}$, E. Padua
,A. Aellia
F. Mellamo

${ }^{I}$ Corso di Laurea in Scienze Motorie, Università Telematica

San Raffaele, Roma, Italia

${ }_{2}^{2}$ Dipartimento di Medicina dei sistemi, Università di Roma Tor

Vergata, Italy

${ }^{3}$ Dipartimento di Sanità Pubblica e Biologia Cellulare, Università di

Roma Tor Vergata, Italy

${ }^{4}$ Corso di Laurea in Scienze Motorie, Università di Roma Tor Vergata, Italy

Objective: Evaluate the effects of whole-body vibration (WBV) in overweight patients on body composition, bone density and anthropometric 
measures and parameters related to glucose metabolism, insulin resistance and systemic inflammation.

Methods: We recruited 23 obese (BMI $>30 \mathrm{~kg} / \mathrm{m} 2$, age $42.5 \pm 13$ years) 9 males and 14 females. Patients were subjected to a characterization of metabolic and hormonal baseline (T0) and then randomized into two arms (WBV group: 12 male subjects 48 females vs GC: 11 subjects 5 males and 6 females) and tested after 2 months (T1) . Dietary therapy was the same for WBV and GC (low-calorie, 55\% carbohydrates, 30\% fat, $15 \%$ protein and fiber $>30 \mathrm{~g}$ ). Specifications of WBV were: frequency $30 \mathrm{~Hz}$, duration 30 minutes session (alternating 1 minute of vibration 1 minute of rest), tri-weekly basis.

Results: All subjects completed the study without complications. Both groups showed weight loss (WBV $4.1 \pm 1.3 \mathrm{p}<0.01$, GC $4.3 \pm 1.8 \mathrm{~kg} p$ $<0.01)$, a reduction in waist circumference $(103.7 \pm 6.1 v s$ WBV $98.1 \pm$ $5.8 \mathrm{~cm}, \mathrm{p}<0.05$, GC $101.4 \pm 5.4$ vs $99.4 \pm 3.8 \mathrm{~cm}, \mathrm{p}<0.05)$ and an improvement in area under the insulin curve after OGTT (AUCinsulina) (WBV $121.8 \pm 56.8$ vs $80.4 \pm 42.1 \mathrm{mcu} / \mathrm{ml} * \mathrm{hp}<0.01,134.4 \pm$ GC 53.8 vs $95.6 \pm 72.1 \mathrm{mcu} / \mathrm{ml} * \mathrm{~h}, \mathrm{p}<0.05)$. For these parameters, however, differences between groups were not significant. WBV subjects showed a significant reduced of plasma leptin levels $(\mathrm{p}<0.01)$ and reduction of fat mass percentage $(\mathrm{FAT} \%, \mathrm{p}<0.001)$ and the amount of fat mass (FAT kg, $\mathrm{p}<0.01$ ), higher than the control group (difference between the two groups $\mathrm{p}<0.01)$.

Conclusions: In obese patients WBV could reduce body fat and improve insulin sensitivity more than dietary therapy alone.

\section{Evaluation of the level of young physical activity in fitness center}

\section{T. Iona and A. Ammendolia}

\section{School of Medicine, University of Magna Graecia, Catanzaro, Italy}

Introduction: Recent studies underlined the importance of an adequate physical activity for the physical and mental development in children and adolescents (Biddle 2004). The knowledge of the anthropometric characteristics and physical abilities is very important for improving training programs for young people in the fitness centers. This study reports basic data referred to physical activity in young population during 4 years of activity (2008-2012).

Methods: 148 children (mean age: $8,49 \pm 2,57$ years; $56 \%$ are girls; $44 \%$ are boys), were enrolled. For all subjects anthropometry testing (height_H, weight_W, BMI \%, weist circumefernce_WC), EUROFIT (flexibility test) and leg measures tests (Weiner and Lourie,1981) were measured at the beginning of their activities (PRE), after 8 hours/lessons (POST_8L) and finally after 3 months of lessons (POST_24L). Statistical analyses including cross-tabling and the analysis of variance were used to analyze the assiociation between physical activity, screen time and subjectively reports.

Results: Same differences were observed after POST_8L and POST_24L in the test WC, but body measures showed negative difference in W, BMI . In the leg measures and flexibility differences statistically significant were noted.

Conclusion: The results show that children who attend a systematic motor activity have a regular grids growth. Objective and subjective measures may reflect different construct and contexts of physical activity in association with anthropometric measures. Present data are useful to realize an adequate training program for young people, based on anthropometric caracteristics.

\section{References}

- Biddle S, Gorely T, Stensel D (2004): Health-enhancing physical activity and sedentary behaviour in children and adolescents. Journal of Sports Sciences 22:679-701

- National Association for Sport and Physical Education. (2004)
Physical activity for children: a statement of guidelines for children ages 5-12. AAHPERD Publications;

- Weiner JS, Lourie JA (1981) Practical Human Biology. Academic Press, London

- Ogden CL, Flegal KM, Carroll MD, Johnson CL. (2002) Prevalence and trends in overweight among US children and adolescents, 19992000. JAMA. 288:1728-1732

\section{Group fitness activities on elderly: an useful approach to prevent} injuries and dropping out

\section{A. Patti ${ }^{1}$, M. Raccuglia ${ }^{1}$, M.C. Cataldo ${ }^{2}$, M. Bellafiore ${ }^{1}$, G. Battaglia , C. Mammina ${ }^{3}$, A. Bianco ${ }^{1,4}$, A. Palma $^{1,4}$}

${ }^{1}$ University of Palermo, Department of Sport and Exercise Sciences (DISMOT)

${ }^{2}$ National Health Service (ASP 6)

${ }^{3}$ University of Palermo, Department of Sciences for Health Promotion "G. D'Alessandro"

${ }^{4}$ Scuola dello Sport, CONI Sicilia, Italia

The aim of this study was to examine the opportunity to adopt already validated function ability rating scales on group fitness activities with the intention of preventing injuries and reducing dropping out of activities. The study was conducted with a quasi-experimental design. The Berg Balance Scale (BBS) and the Barthel index (BI) were administered to a Control Group (CG) of elderly and a Sperimental Group (SG) of elderly as well. SG consisted of four classes of ballroom dancing. Participants were invited to answer to BI questionnaire and afterwards to carry out the BBS under supervision of a specialist. The exclusion criteria were: for CG and EG 1) persons with less age than 65 years old; 2) persons with a positive diagnosis of disease; 3) ex-professional athletes; 4) moreover for SG, no more than 3 months of ballroom dancing experience. At this stage, an excel file with means and SD were used to report preliminary descriptive results. We fixed a minimumgrade of 7 ! $0 \%$ for both scales (BBS-70\% and $\mathrm{BI}-70 \%$ ) as score able to confirm our hypothesis. 122 people participated to the study. We recruited 47 people as CG $(74,3 \pm 5,4$ yrs. $26,8 \pm$ $4,4 \mathrm{BMI})$ and 75 people as $\mathrm{SG}(73,0 \pm 5,6 \mathrm{yrs} 26,1 \pm 3,9 \mathrm{BMI})$. The BI and $\mathrm{BBS}$ of $\mathrm{CG}$ were $76,70 \pm 33$ and $30,9 \pm 14,9$ respectively, while the $\mathrm{BI}$ and BBS of SG were 98,13 $\pm 7,0$ and 50,5 $\pm 5,4$ respectively. The BI of CG shown 0,29 K-S value while the same analysis for SG shown 0,49. We found the same trend on BBS values of CG $(0,07)$ compared to the BBS of SG $(0,16)$. In SG the BBS-70\% shown $96,0 \%$ of cases compared with $27,6 \%$ of cases of CG. The BI indicated a very similar profile than BBS. In SG the BI-70 shown $98,6 \%$ of cases while the BI-70 of CG shown $70,2 \%$ of cases. Moreover, $36,0 \%$ of SG reported falls during the previous six months while $\mathrm{CG}$ reported $53,2 \%$ of fall in the same period of time.The BBS values of $\mathrm{SG}$ who reported falls was $49,4 \pm 5$ ! ,8 with a value of $\mathrm{BI}$ corresponding to $97,0 \pm 9,8$. The $\mathrm{CG}$ w ho reported falls shown $\mathrm{BBS}$ values of $26,7 \pm 14,8$ and $\mathrm{BI} 67,8 \pm 36,0$, respectively.

BBS and BI seem to be able to predict who are eligible to start with Group Fitness and who are not. The majority of the cases of SG reported BBS and BI values higher than $70 \%$ of maximal score. On the other side the $\mathrm{CG}$ shown opposite values of BBS and BI. As expected, BBS seems to better predict than BI. A large scale of cases and an accurate statistical analysis should confirm those findings.

\section{A specific training protocol for the elderly}

\section{S. Pignato, R. Schembri, V. Patania, P. Mango, M. Lipoma}

Facoltà di Ingegneria, Architettura e Scienze motorie, 
Università di Enna Kore, Enna, Italy

Introduction: The present research examined the influence of specific training, based on conditional and coordinative motor abilities, in adults aged from 65 to 75 . The main aim of this research was to lead subjects through modification of sedentary lifestyle factors, that account for the largest numbers of deseases, such us obesity, arterial hypertension, cardio-vascular pathologies.

Objectives: The objectives of this study were:

- to compare, at baseline assessment, physically active subjects with sedentary controls, according to gender;

- to verify, at the end of a specific training protocol, possible differences between physically active subjects and sedentary controls, according to gender.

Method: Participants $(n=20)$ were divided into two groups: Active subjects ( 5 men and 5 women), who were regularly involved in physical activities (Experimental Group), and Inactives ( 5 men and 5 women) who, conversely, were not physically actives (Control Group).

Intenvention was arranged for a month, a three-times-per-week. Subjects trained for 60 minutes each time.

The baseline assessment, the intervention, and the final assessment were carried out in a gym.

To measure motor abilities were used the following Motor Tests, validated by S.I.G.G, Gerontology and Geriatry Italian Society:

- Tinetti Balance and Gait Scale

- Up \& Go Test

- 30 sec. Chair-Stand Test

- $30 \mathrm{sec}$. Arm Curl Test

- Back Scratch Test

- Chair Sit and Reach Test

Results: Comparisons between baseline and final assessment showed that in Experimental Group, final scores were higher than baseline ones. Females scores increased 2 points in all of the test, males scores increased 3 points in $30 \mathrm{sec}$. Chair Stand Test.

In Control Group, females' final scores were lower in $30 \mathrm{sec}$. Arm curl test and Chair Sit and Reach Test than baselines ones; Males in Control Group not had any change in final scores, except in Back scratch test and Chair sit and reach test.

Conclusions: Findings showed that physical activity enhanced the muscle strength in the arms and legs, the balance, and the flexibility in Experimental Group subjects.

Conversely, a sedentary life style affected anatomical and physiological parameters in women of Control Group. In Control Group men, sedentary behaviors limit changes torward an healthier lifestyle. Finally, physical activity provides a valid tool to reduce degenerative processes of aging.

\section{A web-based survey about protein supplementation amongst net-surfers. An innovative approach using Protein Project Google App ${ }^{\odot}$}

\section{Raccuglial, G. Caramazza3, C. Mammina 2, M. Bellafiore ${ }^{1,3}$, G. Battaglial ${ }^{1,3}$, R. Palma ${ }^{1}, A$. Bianco ${ }^{1,3}$, Antonio Palma ${ }^{1,3}$ \\ ${ }^{I}$ University of Palermo, Department of Sport and Exercise Sciences (DISMOT), Palermo, Italy \\ ${ }^{2}$ University of Palermo, Department of Health Promotion \\ "G. D'Alessandro", Palermo, Italy \\ ${ }^{3}$ Scuola dello Sport, CONI Sicilia, Italia}

Web surveys present methodological challenges including lower response rates as compared to other survey methods like face to face interview, self reports using questionnaire and phone interview. The effi- cacy and appropriateness of design elements of invitations to participate in a web survey is not yet well understood for many reasons. To understand the feasibility of this innovative approach, an experiment (Protein Project) with key questions regarding protein supplementation was set up according to previous findings (Goston et al. 2004; Bianco et al. 2011). A Google App (C) was used to generate a questionnaire with seven-items and subsequently the created form was redirected to various social networks and fitness/wellness web communities (fitnessa360.com; iarfonline.com; facebook.com; twitter.com). The target of the Protein Project was 20/50 years old net-surfers (NS) that voluntarily and phishing free, visited the webpage and answered the questions. At this stag! e $326 \mathrm{NS}$ participated to the study. As expected, the majority of them were male $(63,40 \%)$ and the range of age more representative was 21-30 years $(70,90 \%)$. Three to five hours $(38,60 \%)$ sitting time was the most chosen interval from NS followed by 5-8 hour interval $(31,20 \%)$. The results show a percentage of 36,20 of protein supplement usage among NS, of these, $38,50 \%$ declare that have autonomously chosen to take them and $32,30 \%$ that have been advised from their instructor. It is interesting to note that despite the methodological approach is new with a low response rate, the results are in line with Goston's and Bianco's findings. What's new in this approach is to collect a huge amount data with low costs, simple and direct procedures. The project at this stage leads to the validation so it needs to achieve the reliability of web page surveys (currently in Italian and English) hoping that future results will still remain in line with what already found in $\mathrm{t}$ ! his pilot.

References

- Bianco A et al. (2011) Protein supplementation in strength and conditioning adepts: knowledge, dietary behavior and practice in Palermo, Italy. J Int Soc Sports Nutr. Dec 29;8(1):25.

- Goston JL, Correia MI (2010) Intake of nutritional supplements among people exercising in gyms and influencing factors. Nutrition 26:604-611

- Morrison LJ, Gizis F, Shorter B (2004) Prevalent use of dietary supplements among people who exercise at a commercial gym. Int J Sport Nutr Exerc Metab 14(4):481-92

\section{The effects of three different training protocols on beginners in eight weeks}

\section{P. Proia, A. Aprile, F. Lo Curcio, A. Bianco, A. Palma}

Dipartimento di Studi Giuridici, Economici, Biomedici, Psicosociopedagogici delle Scienze Motorie e Sportive, DISMOT, Università degli Studi di Palermo, Palermo, Italia

The aim of this study was to compare three different methods of training in order to determine the effectiveness on weight loss in relation to body composition in adults.

One hundred subjects aged 18 to 38 years, with a BMI equal to or greater than 27 and a body fat percentage greater than $22 \%$ for women and $12 \%$ for men, were enrolled in this study. They were subdivided into 3 groups that followed a different protocol.

Before to start the training protocols was detected the starting level of fitness by a test of maximal oxygen consumption, $\mathrm{VO} 2$ max and maximal strength tests that it was detected by one maximal repetition of the major muscle groups; this has allowed to draw up personalized training cards, with specific loads for each participant of the project. However, to detect the health conditions blood tests were made. Particularly, we checked glucose, BUN, total cholesterol, triglycerides, HDL and LDL as well as CK and LDH (markers of health status of the muscles).

The three different training protocols are called ERE (EnduranceResistance-Endurance), ECE (Endurance-Circuit-Endurance) and CIRCUIT TRAINING. 
The ERE protocol included three parts: the first and the third phase were aerobic in which the heart rate was $65 \%$ of the maximum. The central anaerobic phase in which there were the involvement of different areas through the use of muscle strength machines. The intensity of the exercises was maintained at $60-70 \%$ of maximal repetitions.

The ECE protocol was different just in the central part, in which it required repetitions of free body exercises with an intensity maintained at $50 \%$ of maximal repetitions. Obviously, the CIRCUIT TRAINING protocol was the central part of the previous protocol.

At the end of the eight weeks of training all the determinations (body composition and blood tests) was made.

The results obtained suggest that the optimum methods for the purpose of improvement of the physical form (increase lean body mass at the expense of fat mass) is Endurance-Circuit-Endurance that is the combination of aerobic and circuit training. Future studies are needed in order to confirm this preliminary results.

The evaluation of motor skills on young soccer players: an innovative approach

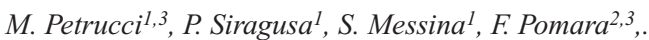 \\ M. Bellafiore ${ }^{1}$, G. Battaglial ${ }^{I}$ A. Bianco ${ }^{1,3}$, A. Palma $^{1,3}$ \\ ${ }^{1}$ University of Palermo, Department of Sport and Exercise Sciences \\ (DISMOT) \\ ${ }^{2}$ Medeor Research Centre, Palermo, Italy \\ ${ }^{3}$ CUS Palermo Lab, Italy
}

It is known that motor skills development should have the role of a control parameter in the entire development of a child; therefore, the studies on this field refer to assessing skills in the early stages of development with the aim of intervening in the sense of learning new movement structures, prevention, and skill development. Testing motor abilities and monitoring their development is difficult, especially when testing children's skills. It involves a high level of accuracy, reliability, and objectivity. To date, in literature a number of motor ability tests are present. The aim of this study was to compare the already validated motor skill tests with a modified Harre ability test also called "Petrucci Ability Test". A number of 56 young male soccer player $(8,94 \pm 0,96 \mathrm{yrs}$; $137,5 \pm 6,8 \mathrm{~cm} ; 34,9 \pm 6,4 \mathrm{~kg}$ ) participated to the assessment of motor skills. Standing broad jump, 20mt sprint, Harre Test and Petrucci Ability Test (PAT) wer! e administered to the participants for a total of three times in a period of three weeks. The best score performed in each test was used for statistic correlations and for a paired $t$ test. The PAT $(15,07 \pm 2,00 \mathrm{sec})$ resulted strongly related to the Harre test $(17,14 \pm 2,7 \mathrm{sec})$ as expected, 0.87 . The results of standing broad jump $(132,13 \pm 28,9 \mathrm{~cm})$ and $20 \mathrm{mt}$ sprint $(4,26 \pm 0,33 \mathrm{sec})$ appear to be in line with standards. Stratifying by age (8-9 yrs and 10-11 yrs) we found a significantly difference between 8-9 yrs Harre tests and 8-9 yrs PAT (0.0001) while no difference were found between 10-11 yrs groups. Pearson correlations have shown similar trend. Preliminary results show an interesting accuracy, reliability and objectivity of PAT, in particular for 8-9 yrs participants. The absence of dive roll in PAT seems to be the key point characterizing the originality of the Test. In conclusion, more studies with a large number of subjects are necessary to confi! rm that PAT is a reliable test for the evaluation of motor skills on y oung soccer players.

\section{Lower limb performance evaluation of professional and young soccer players}

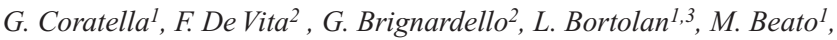
G. Corradini ${ }^{2}$, N. Rinaldo ${ }^{1}$, F. Schena ${ }^{1,3}$
${ }^{1}$ Department of Neuroscience and Kinesiology, University of Verona, Verona, Italy

${ }^{2}$ Technical \& Medical Staff, AC ChievoVerona, Verona, Italy

${ }^{3}$ CeRiSM, University of Verona, Rovereto Italy

Aim: Evaluation of soccer players physiological measurements is important to understand overall fitness of each athlete and to avoid injuries due to muscular imbalances. Since 2010 Sports Science Faculty of University of Verona and A.C. Chievo Verona collaborate to assess anthropometric and physiological tests on main and youth team athletes in order to check potential imbalances or physical problems. Therefore aim of project is to verify differences between First League and youth team, to detect within subjects imbalances and to correlate physiological and performance measures.

Methods: A total of 104 evaluations have been concluded, considering First League team (54) and youth team (48). Knee extensors and flexors concentric and eccentric peak torque $\left(60^{\circ} / \mathrm{sec}, 85^{\circ}\right.$ as ROM) has been measured using Cybex isokinetic dynamometer. Functional ratio has been calculated as ratio between hamstring eccentric peak torque and quadriceps concentric peak torque (Delextrat 2010). Jump height and single limb force development during jump have been measured using force platform. One way ANOVA has been used to detect differences between groups, while correlation among variables has been detected using Pearson coefficient.

Results: A good lateral balance resulted both in main team and in youth team in knee extensors strength ( 0.9 N,CI95\% $-1,9$ to 3,7, n.s.) and knee flexors $(0,8 \mathrm{~N},-24$ to 23 , n.s.).Quadriceps strength was significantly greater in main team compared to youth team ( $43 \mathrm{~N}, 17$ to $70, \mathrm{p}<0,01)$, no difference appeared in knee flexors strength, while $\mathrm{H} / \mathrm{Q}$ functional ratio resulted higher in latter ( 0,05 A.U., 0,023 to $0,093, p<0,01)$. Significant correlation was found between single limb knee extensors peak torque and single limb force developed during jump ( $\mathrm{dx} 0,630, \mathrm{p}<0,05$ and $\mathrm{sx}$ $0,724, p<0,05)$. Bilateral knee extensors peak torque and jump height was not significantly correlated.

Conclusion: Nonetheless dominant limb is involved much more in kicking and dribbling, no differences appeared between legs. Classical soccer training improves quadriceps strength, as shown by greater knee extensors force found in main team. Consequently, decrease of functional ratio should suggest an enforcement of knee flexors, due to the use of functional ratio as marker of hamstrings strain injury odds. Jump performance seems to be not totally dependent on lower limbs strength, even if strength influences the ground reaction forces.

Delextrat A. et al, The use of the functional H:Q ratio to assess fatigue in soccer. Int J Sports Med. 2010 Mar;31(3):192-7. Epub 2010 Feb 15.

\section{Accuracy of Sensewear Armband ${ }^{\circledR}$ in estimating flat $v s$ inclined pole walking energy expenditure}

\section{G. Vernillo ${ }^{1,4}$, A. Leonardi ${ }^{1}$, A. Grainer ${ }^{2}$, C. Doria ${ }^{3}$, A. Savoldelli ${ }^{1}$, C. Reggiani ${ }^{2}$, G. Fanò-Illic ${ }^{3}$, V. Verratti ${ }^{3}$, F. Schena ${ }^{1,4}$}

${ }^{1}$ CeRiSM, University of Verona, Rovereto.

2 Physiology Lab, Department of Biomedical Sciences, University of Padua Padova, Italy

${ }^{3}$ Department of Neuroscience \& Imaging, University "G. d'Annunzio", Chieti-Pescara, Italy

${ }^{4}$ Department of Neuroscience and Kinesiology, University of Verona, Verona, Italy

Aim: The interest in the determination of metabolic response during different life style conditions has showed in the last years a wide increase specially related to the availability of portable devices which can be applied in many living situation. Walking is a very common physical ac- 
tivity which regularly includes flat or slope path. The purpose of this

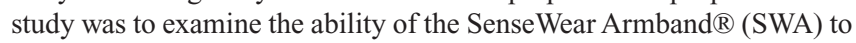
accurately assess energy expenditure (EE) during laboratory treadmill walking with poles at different slope.

Method: 7 experienced women trekkers (age $34.75 \pm 6.89 \mathrm{yrs}$; body mass $62.70 \pm 5.86 \mathrm{~kg}$; adjusted body mass $72.23 \pm 5.77 \mathrm{~kg}$; height $163.50 \pm 6.38 \mathrm{~cm}$ ) were recruited. In a cross-sectional fashion, after 4 min of basal sampling subjects performed in a randomized-counterbalanced order $4 \mathrm{~min}$ at 3, 4, 5 and $6 \mathrm{~km} \cdot \mathrm{h}-1$ at $0 \%$ slope and $4 \mathrm{~min}$ at 3 $\mathrm{km} \cdot \mathrm{h}-1$ at 5,10 and $15 \%$ slope. During the entire protocol subjects wore simultaneously SWA and a K4 b2 portable metabolic system (K4, Cosmed, Italy) combined with a $6 \mathrm{~kg}$ rucksack and trekking poles. The adjusted body mass $(\sim+9 \mathrm{~kg})$ was always taken into account for the specific calculations. The last minute of each step were used to compute a caloric value ( $\mathrm{kcal} \cdot \mathrm{min}-1)$ which was the average $\mathrm{O} 2$ and respiratory exchange ratio for K4; while, for SWA the information from the sensors, together with gender, age, height, and weight, are incorporated into proprietary algorithms. All data were t! ime-matched to within $1 \mathrm{~s}$ of K4. The normal distribution of the data was confirmed using the Kolmogorov-Smirnov test. Paired-sampled t tests were used to determine differences in EE values between the two devices. Bland-Altman plots were drawn to establish the bias of the EE values, assuming K4-EE value as criterion variable.

Results: Data are presented as means \pm SD and the -level was set at $\mathrm{p}<$ 0.01 . The SWA significantly overestimated $(p<0.001)$ the EE of flat walking (by $0-34.57 \%$ ) and corresponding to 5.47 (2.16) Kcal•min-1. While, the SWA significantly underestimated $(\mathrm{p}<0.001)$ the EE of inclined walking (by 16.43-46.05\%) and corresponding to 4.43 (2.09) Kcal•min-1. Thus, EE-SWA was significantly higher than EE-K4 at flat walking conditions; while it was significantly lower than EE-K4 at inclined walking conditions.

Conclusions: The results of the present study seem to indicate that SWA, in the tested conditions, do not guarantee an accurate a stable measurement of EE in different walking condition. More specifically it cannot be applied, without previous calibration with direct energy expenditure determination when the slope of the walking path has frequent and short lasting changes.

\section{ORAL PRESENTATIONS}

\section{Saturday, October $6^{\text {th }}, 2012$}

\section{Session 5.3. Physical Activity \& Health}

\author{
The role of physical education in the choice of the lifestyle \\ in student at the end of secondary school: preliminary \\ interpretation
}

\section{Lanza, I. Salvadori}

Department of Neurological, Neuropsychological, Morphological and Movement Sciences. University of Verona, Italy

Physical education can be a powerful influence on promoting youth physical activity (Malina, 2001, Wallhead, 2004 - Pate, 2006) but this will not necessarily result from participation, per se: the effects are likely to be mediated by the nature of the interactions among students and their teachers, parents and coaches (Bailey, 2006). In this study we examined some characteristics (BMI, physical activity level, self efficacy and evaluation in Physical Education) of the students involved in the fifth year of the secondary schools and the role that they attribute to Physical Education (PE) in order their lifestyle. The interaction among factors will be analyzed subsequently.

Methods: Four hundred eighteen students (Females: number $=220$; age $=18.55 \pm 0,61$ years - Males: $\mathrm{n} .=198$; age $=18.77 \pm 0.81$ ), recruited in three Veneto towns, have correctly compiled an articulated questionnaire that explored anthropometric and personal data, Physical Activity (IPAQ-A), Physical Self Efficacy (PSE scale) and the average of their evaluation in PE in the last two years (EV). Five questions related to the assessment that students give to the Physical Education and its teachers (Nuviala, 2011) were also proposed. In these questions students could choose between the options "strongly agree", "agree", "disagree" and "strongly disagree". T. test, Mann-Whitney Rank Sum Test, Pearson correlation were used to analyze parametric data and "Chi square" for nonparametric data.

Results: Males show higher values than females $(p<0,001)$ in Age, BMI, IPAQ-A, PSE and EV. In females there is a weak correlation between PSE and BMI/IPAQ-A/EV $(r=-0.15, \mathrm{p}<0.05-0.36, \mathrm{p}<0.001-0.42$, $\mathrm{p}<0.001$ respectively) and between EV and IPAQ-A $(r=0.16, p<0.05)$. In males there is a weak correlation between IPAQ-A and BMI/EV/PSE $(\mathrm{r}=0.17, \mathrm{p}<0.05-0.33, \mathrm{p}<0.01-0.32, \mathrm{p}<0.001$ respectively $)$, between EV and PSE $(r=0.41, \mathrm{P}<0.001)$ and between Age and EV $(r=-0.2$, $\mathrm{p}<0.01$ ).

Males and females answered to the five questions about PE without differences: almost $80.0 \%$ agree that "Physical Education classes are fun" and that "Physical Education is important for my own personal development"; the 68.7\% agree that "You don t waste time in Physical Education classes"; the 58.4\% agree that "Physical Education teaching staff help me become interested in physical activity and recreational sports"; only the $47.4 \%$ of the students agree that "Physical Education classes help me engage in the practice of sport during spare time".

Discussion: Data seems to confirm (Spence, 2010) greater values physical self efficacy and in physical activity in males. Should be investigated more thoroughly motivation about the higher evaluation of males than females in PE. If will be confirmed the limited number of student that attribute a significant role to PE for the adoption of their lifestyle, the problem should be considered in the PE guidelines and in PE teacher training. 
Relationship between performance and heart rate variability in amateur basketball players during playoffs

\author{
S. Di Fronso ${ }^{1,3}$, G. Delia ${ }^{1}$, C. Robazza ${ }^{1,2}$, L. Bortoli $^{1,2}$, M. Bertollo $^{1,2}$ \\ ${ }^{1}$ Behavioral Imaging and Neural Dynamics (BIND) Center, \\ D'Annunzio University, Chieti, Italy \\ ${ }^{2}$ Dept Medicine and Aging Sciences, D'Annunzio Univ., Chieti, Italy \\ ${ }^{3}$ Dept Biomedical Sciences, D'Annunzio University, Chieti, Italy
}

Aim: The purpose was to investigate the relationship between Heart Rate Variability (HRV) and performance in players of a basketball team during playoffs. HRV has become the conventionally accepted term to describe variations of both instantaneous heart rate and R-R intervals (Task Force, 1996). The cardiovascular system is mostly controlled by autonomic regulation through the activity of sympathetic and parasympathetic pathways of the autonomic nervous system. Analysis of HRV permits insight in this control mechanism. It can be easily determined from ECG recording resulting in time series (R-R intervals) that are usually analyzed in the time and frequency domains (Aubert, Seps, \& Beckers, 2003). In particular, High Frequency (HF) power, derived from a frequency domain analysis, is considered to be reasonably linked to vagal activity (Malliani, 1999; Task Force, 1996).

Methods: Participants were 11 basketball D-level players aged 19-26 years $(M=21.9, S D=3.1)$ involved in the playoff phase of the competitive season, but only 7 athletes completed the data collection due to injuries. HRV monitoring lasted seven weeks and was conducted in a silent room of training facilities in the morning of the match day. HRV was computed from 10 minutes of resting electrocardiogram recorded via BioHarness, using ADInstruments HRV Module, and the data were transformed into their natural logarithm ( $\mathrm{Ln})$, because of skewed distribution. Athletes' performance was statistically calculated by the team technical staff throughout every match using the procedure developed by the Italian Basket Federation.

Results: Stepwise multiple regression analysis was conducted to test if the HRV indexes in the frequency domain (VLF, LF and HF) significantly predicted participants' level of performance. Regression analysis results showed that the HF accounted for the $15 \%$ of the variance, $\mathrm{R} 2=.15, \mathrm{~F}(1,47)=5.22, \mathrm{p}<.05$.

Conclusions: Findings of this study suggest that vagal activity, expressed by HF index of HRV, can be positively related to the athletes' performance. In particular, higher values of HF index during the morning of the match were associated with higher levels of athletes' performance during the game.

\section{References}

- Aubert, A. E., Seps, B., Beckers, F. (2003) Heart Rate Variability in athletes. Sports Medicine, 33: 889-919

- Malliani, A. (1999) The pattern of sympathovagal balance explored in the frequency domain. News in Physiological Sciences, 14: 111-117

- Task Force of the European Society of cardiology and the North American Society of pacing and electrophysiology (1996) Heart Rate Variability: standards of measurement, physiological interpretation and clinical use. Circulation 93: 1043-1065

\section{Physiological response on $1 \mathrm{RM}$ bench press performance}

\section{E. Thomas ${ }^{1}$, D. Filingeri ${ }^{2}$, S. Vasile Simone ${ }^{1}$, T. Moro ${ }^{3}$, E.Mancuso $^{1}$, A.Palma ${ }^{1,4}$}

${ }^{1}$ University of Palermo, Department of Sport and Exercise Sciences (DISMOT), Italy

${ }^{2}$ Environmental Ergonomics Research Centre, Loughborough

University, $U K$
${ }^{3}$ University of Padua, Department of Biomedical Science, Italy ${ }^{4}$ Scuola dello Sport, CONI Sicilia, Italy

A number of methods for determining upper body maximal strength are present. The 1-RM is defined as the maximum amount of resistance that can be performed for only 1 repetition and it has been shown to be reliable in various populations. Further, setting an intensity of resistance training based on a percentage of the 1-RM has been found to be effective for improving outcomes such as muscle strength, muscle power, and muscle hypertrophy. Little is known about the metabolic cost of $1 \mathrm{RM}$ evaluation and which is the optimal recovery time between sessions. We analyzed the physiological response during a standardized protocol of 1RM-Bench Press Test (1RM-BP). Fourteen healthy subjects $(25,6 \pm 7,4 \mathrm{yrs} ; 166,3 \pm 0,9 \mathrm{~cm} ; 65,6 \pm 13,2 \mathrm{~kg})$ performed a $1 \mathrm{RM}-\mathrm{BP}$ following standardized procedures. VO2 and Heart Rate ware measured before, during and after the BP performance while Systolic (Sys) and Diastolic (Dys) pressure were measured only before and after the test. At the e! nd of the 1RM-BP, Blood Lactate (BL)was assessed and participants were asked for perceived exertion according with the Borg Scale (RPE). The protocol consisted of five steps; 15 repetitions with of the subjects body weight were performed in the first step, subsequently weight was added up to $1 / 2$ of bodyweight and a maximal number of repetitions were performed. In the third step the participants executed the theoretical 1RM-BP according to the Mayhew's formula that considers the number of repetitions completed with $1 / 2$ bodyweight of each subject. The other steps ware performed only if the third one was completed, if not $2-5 \%$ more weight was added on the barbell until 1RM was obtained, this was repeated if necessary for a maximum of other 2 times. The results show that there are no significant differences in $\mathrm{VO} 2$ values between male and female, only in the second step ( $1 / 2$ Bodyweight $)$ males achieve higher results $(\mathrm{p}=0,0032)$. BL was significantly! higher in male $(p=0,042)$ even if \%Lean mass was not quite significantly higher than female $(p=0,0532)$. The most interesting result is shown by Pearson's correlation between \%Lean mass $v s$ RM-BP; male were positively correlated( $\mathrm{r}=0,61)$ while female were negatively $(\mathrm{r}=-0,65)$.

Unexpected results need to be confirmed by a larger number of participants, but at this stage this study shows that in any case there are gender differences. Furthermore, this study confirms the reliability, feasibility and safety of the 1RM-BP.

\section{A web-based surveillance system on adolescents' lifestyles and obesity prevention: ASSO-FTB preliminary findings. The ASSO Project}

M. Raccuglial, ${ }^{1,2}$, G. Tabacchi ${ }^{2,3}$, E. Thomas $^{1}$, D. Filingeri ${ }^{2,5}$, C. Mammina ${ }^{2,3}$, M. Jemni ${ }^{2}$, A. Bianco ${ }^{1,2,4}$, A. Palma ${ }^{1,4}$

${ }^{1}$ University of Palermo, Department of Sport and Exercise Sciences (DISMOT), Italy

${ }^{2}$ Ministry of Health, The ASSO Project

${ }^{3}$ University of Palermo, Department of Health Promotion

“G. D'Alessandro", Italy

${ }^{4}$ Scuola dello Sport, CONI Sicilia, Italy

${ }^{5}$ Loughborough University, UK

In a context where obesity represents a cause of concern in childhood and where there is a lack of standardized data collection systems, ASSO Project (Adolescents and Surveillance System for Obesity prevention), financed by the Italian Ministry of Health, aims to develop a surveillance system structured on adolescents lifestyles. It is a prospective study with online data collection, simultaneous descriptive data analysis and real time report. The ASSO-Toolkit is made of questionnaires, forms and a fitness test battery: ASSO-PIQ (Personal Information Questionnaire); 
ASSO-PASAQ (Physical Activity, Smoke, Alcohol, Questionnaire); ASSO-FHQ (Food Habits Questionnaire); ASSO-FFQ (Food Frequency Questionnaire); ASSO-BFMF (Body and Fitness Measures Form); ASSO-FTB (Fitness Tests Battery). The aims of this study is to evaluate the predictive validity, the criterion validity and the reliability of ASSOFTB, in other words to assess how well the system operates to meet its obje! ctives.

The ASSO-FTB consists of 20mSRT (Shuttle Run Test) to estimate cardiorespiratory fitness, the handgrip strength test and the standing broad jump to assess musculoskeletal fitness, the $4 \times 10 \mathrm{mSRT}$ to assess motor fitness and last but not least the sit up test to estimate muscle endurance. Forty male adolescents $(17,32 \pm 1,0 \mathrm{yrs} ; 175,3 \pm 6,0 \mathrm{~cm} ; 72,4 \pm 14,7 \mathrm{~kg}$; waist circ. $86,2 \pm 12,4 \mathrm{~cm}$ ) attending the same secondary school, voluntarily participated to the pilot study. In a school setting, the already standardised procedures were administered by the ASSO-FTB specialist in collaboration with the (PE) teacher. All data collected were updated through the already existing website (www.assoproject.info) and codified by the dedicated ASSO-FTB server. All equipments adopted were selected according to the previous publications (HELENA Study, AVENA Study, FITNESSGRAM and ALPHA Study). Data were analyzed using STATISTICA software, two-tail paired t test, ! two-tail Unpaired t test and Pearson correlation were used when appropriate.

Participants were significantly higher in BMI compared to data published by Espana-Romeo et al in 2010. As expected, the maximal isometric strength showed no differences between $\mathrm{dx}(35,07 \pm 7,3 \mathrm{~kg})$ and $\mathrm{sx}$ $(35,8 \pm 4,8 \mathrm{~kg})$ handgrips respectively. The standing broad jump $(178,07$ $\pm 26,9 \mathrm{~cm})$ has evinced less distance than Espana-Romeo study $(183,4 \pm$ $32,75), p=0,37$. The sit-up test reported a mean value of $44,9 \pm 32,8$ reps. On the whole findings show that there is a correlation between handgrip and standing broad jump $(r=0.7)$, underlining that this last test is better indicative of the upper and lower body muscular strength.

The trial showed that the FTB-system was structurally simple, low cost, reliable and well accepted by the key partners. At this stage ASSOFTB seems to be able to meet scientific standards. More studies are necessary for the ASSO-toolkit validation. Preliminary results are very encouraging.

\section{Nutritional status and physical activity in the school-age population of a Sicilian town}

G. Napoli ${ }^{2}$, C. Mammina ${ }^{1}$, E. Amodio ${ }^{1}$, R. Asciutto ${ }^{2}$, C. Costantino ${ }^{2}$, M. Di Pasquale ${ }^{3}$, G. Maringhini ${ }^{2}$, M. Morici ${ }^{3}$, A. Palmeri ${ }^{2}$, S. Parisi ${ }^{2}$, G. Tabacchi ${ }^{1}$, B. Trobia ${ }^{2}$, G. Calamusal

${ }^{1}$ Section of Hygiene - Dept of Science for Health Promotion

"G. D'Alessandro" - University of Palermo, Palermo, Italy

${ }^{2}$ School of Hygiene and Preventive Medicine - University of Palermo, Palermo, Italy

${ }^{3}$ Specialist in Hygiene and Preventive Medicine

The aim of this study was to characterize nutritional status and physical activity in a preadolescent population. Methods. The survey was carried out in the period 2011-' 12 on 891 students enrolled in primary (classes III, IV and V) and secondary schools in Castellammare del Golfo (TP). All data (anthropometric, school and extracurricular activities, eating habits and self-perception of nutritional status) were collected, after obtaining parental consent, through a questionnaire. Results. A total of 697 questionnaires were obtained of which 349 from the primary school $(\mathrm{M} / \mathrm{F}=1.14$, median age $=9.2 \mathrm{y})$ and 348 from the secondary school $(\mathrm{M} / \mathrm{F}=0.92$, median age 12.3y). According to the Cole BMI cut-off points, overweight $(29.9 \%$ vs $26.0 \%, \mathrm{p}=0.29)$ and obesity $(11.7 v s 9,6 \%$ of $\mathrm{F}, \mathrm{p}=0.19$ ) were more frequent, but not significantly, in the male gender. Obesity was more prevalent in primary than secondary school $(13.2 \%$ vs $8.3 \%, \mathrm{p}=0.02) .74 .7 \%$ of the interviews used public or private transportation to go to school, only $25.3 \%$ moving on foot or by bicycle with no significant differences by sex and school level. $11 \%$ of respondents did not exercise at school, the median number of hours being in the primary school 1 hour/week $(\mathrm{h} / \mathrm{w})$ and in the secondary one $2 \mathrm{~h} / \mathrm{w}$. The time spent in sedentary leisure activities (e.g. watching TV and navigating internet) was higher among normal-weight boys of the secondary school (mean, $20.1 \mathrm{~h} / \mathrm{w}$ ) and lower in the girls of the primary school (mean, 15h/w). The average hours spent in extra school sport activities was higher in $\mathrm{M}(\mathrm{M}, 4.4 \mathrm{~h} / \mathrm{w}$ vs $\mathrm{F}, 3.1, \mathrm{p}=0.000)$, in secondary school students (primary, 2.9, secondary $=4.5, \mathrm{p}=0.000$ ) and among them in the overweight-obese children $(6.0 \mathrm{~h} / \mathrm{w})$. Bad nutrition habits (skipping meals and eating $<1$ time/day fruit! and vegetables) were more common in obese $\mathrm{M}$ of the secondary school (91.7\%). Daily consumption of sugary drinks was less prevalent in overweight-obese $\mathrm{F}$ of both school levels (11.7\% and $12.9 \%)$, but more frequent $(22.8 \%)$ in normal-weight $\mathrm{F}$ and overweight $\mathrm{M}(22.1 \%)$ of the secondary school. About self-perception of body weight, it matched satisfactorily with BMI levels estimated from the reported anthropometric data, mainly in the girls and the older students. Conclusions. By taking into account the limits due to the reported anthropometric data, our survey depicts a population with a concerning health risk. The leisure sedentary activities were as diffuse as exercise, which seems to depend more upon environmental/cultural factors linked in turn to age and gender. Eating habits seem to play a role in promoting overweight in the older boys. Poor awareness of overweight appears to influence, maybe with the influence of familiar determinants, body weight excess in boys of the primary school.

\section{Improved mental representation of space in beginner orienteers}

\author{
F. Fischetti ${ }^{1}$, A. Notarnicola ${ }^{1}$, G. Vicenti ${ }^{1}$, L. Laricchia ${ }^{1}$, \\ R. Guastamacchia ${ }^{1}$, S. Tafuri ${ }^{2}$, B. Moretti ${ }^{l}$
}

${ }^{1}$ Dipartimento di Neuroscienze ed Organi di Senso, SAMS Corso di Laurea in Scienze delle Attività Motorie e Sportive, Università di Bari, Italy

${ }^{2}$ Dipartimento di Scienze Biomediche, Sezione di Igiene, Università di Bari, Italy

Aims: The purpose of the present study was to monitor any improvement in orienteering skills attributable to acquiring a better mental representation of space.

Methods: Two groups were examined: the experimental group, who attended 6 mo. of orienteering lessons, versus the control group, who did jogging training instead. Each group, consisting of 20 children, was tested on the Corsi Block-tapping Test, run Forward and Backward, and the Star-Butterfly Test. Pre- and post-tests were administered. In the experimental group, scores increased in mean complexity from pre- to posttest on the Forward and the Backward Corsi tests, while on the StarButterfly Test both time and mistakes had decreased after the training. In the control group, mean complexity and Star-Butterfly Test scores were unchanged from pre- to post-test.

Results: These results showed that after continual training in orienteering techniques, the orienteering group was able to remember and repeat sequences of events with greater precision than before the training, while these skills were unchanged in the control group after training in jogging. 
Post-chemotherapy physical fitness level on children with diagnosis of leukemia: results of a pilot study

\author{
A. Patti ${ }^{1}$, S. Di Trapani ${ }^{2}$, P. Guadagna ${ }^{3,4}$, M.C. Maggio ${ }^{4}$, G. Corsello ${ }^{4}$, \\ P. D'angelo ${ }^{3}$, G. Caramazza ${ }^{2}$, A. Bianco ${ }^{1,2}$, A. Palma $^{1,2}$ \\ ${ }^{1}$ University of Palermo, Department of Sport and Exercise Sciences \\ (DISMOT), Italy \\ ${ }^{2}$ School of Sport (CONI Sicilia), Italy \\ ${ }^{3}$ Pediatric Hematology and Oncology Unit, Oncology Department, \\ A.R.N.A.S. Civico, Di Cristina and Benfratelli Hospitals, Palermo, \\ Italy \\ ${ }^{4}$ Mother-Infant Department, Pediatrics Clinic. University of Palermo, \\ Italy
}

Introduction: Children with diagnosis of leukemia are at risk for developing neuromuscular and musculoskeletal complications such as decreased muscle strength, impaired gross and fine motor performance, decreased energy expenditure, osteonecrosis, and osteoporosis. These secondary complications due to the medical interventions may lead to activity limitations and participation restrictions in daily life experiences. However, few Authors suggest that if children participate in an individualized physical therapy exercise program, these limitations may be prevented or at least reduced. We evaluated the level of physical fitness on children with diagnosis of leukemia (C-LK) during the post-chemotherapy period.

Subjects and methods: Nine $(8,3 \pm 2,7$ yrs $)$ C-LK of both sex participated to the study with the consents of their parents. We also recruited a group of thirty-two healthy children of both sex with similar age $(8,12 \pm 1,56 \mathrm{yrs})$ as control group (GC). In order to assess the fitness level of the experimental sample we used few fitness tests already validated by scientific papers. Standing broad jump, 4 x $10 \mathrm{~m}$ shuttle run test, sit up test and handgrip strength test were administered three times in a period of two weeks during the period of May 2012. Exclusion criteria for C-LK were at least six months of therapy stopped and for CG no regularly physical activity participation. The scores coming from the best performances were used for statistical purpose.

Results: Preliminary results shown no differences in standing broad jump performances. C-LK reported a mean value of $112,55 \pm 32,80 \mathrm{~cm}$, while $\mathrm{CG}$ reported $124,18 \pm 22,19 \mathrm{~cm}, \mathrm{p}=0.11$. An interesting findings is coming from $4 \times 10 \mathrm{~m}$ shuttle run test. The C-LK group performed the test in a mean value of $16.27 \pm 0.83$ seconds, while the control performed the same test in $14.02 \pm 0.25$ seconds, $p=0.001$. No differences between groups were found when comparing sit-up performances $(0.25)$ and handgrip performances ( 0.08 left arm and 0.15 right arm), respectively. Conclusions: The level of physical fitness of C-LK group seems to be in line with control group results. These preliminary data are confirming the eligibility of C-LK to start with specific exercise programs in order to reduce cardiovascular and muscular complications and improve quality of life. The only concern is about the agility test (4 x 10 SRT) who shown a very high significant differences, 2 seconds in $40 \mathrm{mt}$ sprint. To date, the sample size is not adequate to make conclusions. More data coming from C-LK groups are necessary to better understand this topic.
Active lifestyle promotion in COPD: preliminary data about recruitment and efficacy of different models of physical activity

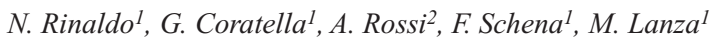

${ }^{1}$ Department of Neurological, Neuropsychological, Morphological and Movement Sciences, University of Verona, Verona, Italy

${ }^{2}$ Cardiovascular and Thoracic Department, Pneumology, ULSS 20

Background: Physical activity (PA) is considered as the most effective non-pharmacological intervention to alleviate symptoms and optimize functional capacity of patients with chronic obstructive pulmonary disease (COPD). However, a considerable proportion of eligible patients declines participation or drop out of PA programs. The aims of the study are to determine the feasibility of recruiting patients with COPD and to access the efficacy of 3 months of different types of PA training models. Methods: Two hundred sixty-nine males patients with COPD were identified from Respiratory Division of ULSS 20, but only 132 patients matched the study inclusion criteria. We were able to contact 92 patients to participate at our study and 37 accepted (mean FEV1 $=66.9 \pm 19.1 \%$ of predicted and $\mathrm{FEV} 1 / \mathrm{FVC}=55.4 \pm 14.1 \%$ of predicted, age $66.35 \pm 4.6$ years). Patients were randomized in a Fitness Center based activities $(\mathrm{FC}=13)$, in a PA Educational $(\mathrm{EDU}=12)$ and in a Control Group $(\mathrm{CG}=12)$. FC patients were involved in a PA program of 3 times/week, 90 min per session of fitness, indeed EDU participated at 10 weeks of low progressive supervised training (Nordic walking, aerobic group class, walking paths and fitness) and motivational sessions. Behavioral and physiological outcomes were reported pre and after 3 months of intervention. Repeated measures ANOVA and analysis of covariance (ANCOVA) were performed to detect the significance of the changes.

Results: No accident occurred during the study; 2 subjects dropped out for illness. CF adherence was $87 \%$ while EDU was $100 \%$. Significant time $\mathrm{X}$ group interaction has been found in 6 minute walk test, upper and lower body strength, flexibility, non dominant lower limb balance and IPAQ. Both in CF and EDU significant improvement were found in upper and lower body strength, flexibility and IPAQ. CF showed significant increases in endurance performance and balance. Post-hoc analysis revealed significant differences between $\mathrm{CF}$ and $\mathrm{CG}$ in 6 minute walk test (15.20\% vs -2.85\%), upper body strength (19.13\% vs -2.35\%) and non dominant lower limb balance (114.55\% vs 17.46); significant differences between EDU and CG were found in upper (15.25\% vs -2.35\%) and lower $(13.25 \% v s-1.08 \%)$ body strength, flexibility $(+5.92 \mathrm{~cm} v s-$ $0.55 \mathrm{~cm})$ and IPAQ $(68.13 \%$ vs $-31.66 \%)$. No differences were found between CF and EDU.

Conclusions: Compare to literature (Faulkner J., 2010) higher adherence ( $36.5 \%$ vs $7 \%$ ) to the program resulted in our study. Primary outcomes confirm the positive effects of PA programs on health related parameters. EDU increments are due to the high motivational support provided by operators and the motor skills learned during training. Moreover self-selected training can be used to improve the share of PA and consequently health status, based on ACSM guidelines about PA support strategies (Cress M.E., 2005). Results in endurance performances suggest that a structured training $(\mathrm{CF})$ should be recommended to alleviate fatigue and dyspnea. 


\section{ORAL PRESENTATIONS}

Saturday, October $6^{\text {th }}, 2012$

\section{Session 5.3. Law, Economic and Social Aspects of Sport and Fitness Activities}

\author{
Reflexivity and football fandom. The impact of social reflexivity \\ on the behaviour of fans
}

\section{Tallarita}

Facoltà di Scienze Umane e Sociali University of Enna, Italy

The main objective of this research project will be to investigate the emerging and under-researched forms of social reflexivity (Archer, 2010; Donati, 2011) of football fandom that evolving spontaneously within groups of fans who regularly attend football matches in order to broaden the knowledge on indited types and the variation of football fan culture. The questions aim at focusing on how evolution of social behaviour occurring spontaneously and on practices of resistance to the culture of modern football and the differences occurring between models detected if there are local policies that may affect regional patterns of behaviour taken by fans. The central focus of paper will be based on the analysis of social behaviour of the fans, the forms of resistance to the culture of modern football and ethical behaviour triggered by the traditional model known as supporter of the violence. It will also be on the social reflexivity influences on the experiences of fans taking a broader epistemological purpose, analysing the new approach to empirical social reflexivity in sociological theory. Socio-cultural studies of sport fans have labelled as Ultras or Hooligans and more recently consumers to leisure experience entertainment (Crawford, 2004; Giulianotti 2002). Research using a qualitative methodology and ethnographic approach will consider two countries: Italy and England and will be enriched by further exploration at the European level. We will consider various forms of aggregation of shared values, thus regarding football fans as a multifaceted phenomenon that is meaningful not only for sport but also for the wider civil society which is structured on ethical behaviour. The manifestations of reflexivity emerge in spontaneous and informal group participations through the games, accompanied by songs, slogans and banners by the exhibition of the symbol representing the identity of the group or even through newspapers (zine e-zines) or the new media. The reflectivity of the fans is represented by a wider participat ion, including the ability to transform and direct the fans to increase social activities and help build social capital (Putnam, 2000; Coleman, 1988; Coalter, 2008; Hoye \& Nicholson, 2007; Seippel, 2006). In this essay social development refers to activities aiming at promoting integration and social inclusion of ethnic minorities, women and children, now that more and more crowd the sidelines of the Italian and international stage, strengthening social cohesion within local communities. We will also analyse how the reflexivity works inside football fans whilst exploring how this affects the nature of modern football fans and the ways in which the fans put their sports experiences into practice, highlighting the features of being playful, spontaneous and creative, in particular the logic of modern marketing and spectacle (Gruneau 1983).

\section{Lifelong learning strategy for sport and active leisure}

\section{S. Digennaro}

University of Cassino and Southern Lazio Department of Human and Social Sciences and Health Epidemiology, Physical Activity, and Life Styles Section

European Obervatory of Sport and Employment, France

The Sport sector is growing fast across the European Union and is currently facing significant challenges in the field of education and employment which need to be considered if the sector wants to achieve its potential and impact on individuals and communities. The sector lacks a clear career structure that is vital to attract the best people and particularly to give graduates the opportunity to find employment. Then, there is often poor communication and co-operation between stakeholders and, frequently, training sits outside the national qualification structure. Consequently the sector is not delivering its potential as an economic and social driver and the education and training systems fail to equip the workforce with the right skills and competencies. In response to that, the European Observatory of Sport and Employment (EOSE) developed a strategy known as $<>$ (LLL Sport Strategy) that aims to: help key stakeholders to anticipate current and future skills needs; promote transparent and flexible education systems; engage main stakeholders from the sport sector; facilitate the link between the worlds of education and employment. The LLL Sport Strategy has been designed to be flexible so that it can be used by a wide range of stakeholders and to achieve a variety of education or employment objectives in the sector. Furthermore it enables the sector to understand better its current set up and status quo, to monitor its strengths and weaknesses, and help national and local stakeholders to better align with the European Qualification Framework (EQF). The methodology has been partially tested in diverse European countries within the project $<>$ (Education and Culture DG, AGREEMENT NUMBER - 2009 - 5146 / $001-001$ ). The main outcomes of the project as well as the main features of the methodology will be discussed.

\section{Sport as a tool for education, training children and adults: a resource for the school and the strategy to work}

\section{F. Perrotta, A. Pannelli}

Dipartimento di Scienze dell'Educazione, Università di Macerata, Italy

Sport is synonymous with competition, conflict, rivalry, proactive and challenge to the last breath with the dominance of its competitive dimension, more crucial to enhance the values related more to the fight and the contrast between the contenders that all "meeting, the comparison and the discovery of self and other. Meeting, discussion and discovery that are the foundation for growth and training of the person, a pedagogy that can guide the subject for the construction and sharing of basic rules for its social life, to discover and experience the values of tolerance, loyalty, solidarity, brotherhood and belonging.

A purpose and an educational process, two concepts that travel in harmony with each other, lead us to understand how the body-movementsports promote self-knowledge, the environment and its possibilities of movement and also contribute to the formation of personality through the 
pupil's knowledge and awareness of the body's identity.

In the common meaning today mean by sport physical activity which is carried on at least three different levels of emotional investment: for competition, for fun, wholesome activities like. These levels are anthropologically and psychologically acceptable if they are found together and integrated with each other, without a reason to prevail over the other.

\section{Sport and Welfare Policies in Europe}

\section{N. Porro}

Dip. Scienze umane, sociali e della salute, University of Cassino and of Lazio, Italy

The presentation shows the main results of a comparative research on the national sports systems in the European Union. The investigation focuses on the crucial role of voluntary non profit sports organizations.

In Europe the sports organizations since the late 19th century historically grounded both in the social sub-system of voluntarism and in the culture of voluntary action. By an organizational perspective they represented the social backbone of the sports system at large. Voluntary sport strongly contributed to the Nation building and gave birth to original experiences of governance.

Later they also represented the collective actors supporting an increasing insertion of sport in the Welfare agenda.

At present the most of European sports clubs are rooted in the non profit private domain, in the Third Sector organizational area and in the Social Economy. Nevertheless, by a sociological point of view, culture and practice of voluntary sport have been everywhere influenced by social changes and by the uprising of new lifestyles, attitudes, preferences and tastes.

So the European sports system constitutes a controversial and differentiated object, still reflecting heterogeneous dynamics of development. They on turn refer to the profile of the Nation building, to specific forms of modernization, to the social stratification etc. In each country role, structure and regulation of the sport system depend on (i) different legal statuses assigned to sport; (ii) various typologies of acknowledgement of the non profit system at large; (iii) organizational histories, to be linked with specific experiences (regarding sports disciplines, network frames, influence of exogenous and endogenous factors), and on (iv) the emerging role exerted since 2007 by EU (White paper on Sport, Lisbon Treaty, de Coubertin Programme).

The social, cultural and organizational landscape of sport for all itself includes many fields of action, sometimes conflicting each other. One can describe it as a political arena according to the classic Benson's definition (1988), implying different actors, several stakeholders and specific priorities and motivations. We can however identify three main organizational sub-systems: (i) grassroots movements, (ii) sport for citizenship associations and (iii) sport for everybody offer system. On turn, changes and trends in sports organizations have been analysed adopting either a socio-historical approach or sociological analysis of organizations according to emerging theories.

The presentation emphasizes the relationship between these conceptual and methodological contributions and the specific role of sport in producing social capital and logics of action inspired, according to Putnam (2001), to both cohesion oriented programmes (bonding model) and to social inclusion (bridging paradigm).

\section{A realist evaluation of an anti-doping programme: regulatory measures and change of gym managers attitudes toward performance-enhancing-substances sale}

\author{
L. Leone ${ }^{1}$, C. Pesce ${ }^{2}$ \\ ${ }^{1}$ Dept Human Motor Science Sport University Roma Foro Italico, \\ Director CEVAS (Rome), Italy \\ ${ }^{2}$ Dept Human Motor Science Sport University Roma Foro Italico, Italy
}

Background: The use of Performance-Enhancing Substances (PES) and dietary supplements among gym attendees has become a widespread public health problem extending from adults even to school-age children. Different programmes, grounded on social-cognitive theories and aimed at changing attitudes and behaviour $\mathrm{s}$ of various target groups (athletes, students, gym attendees) have been developed, but their evaluations resulted in uncertainty or lack of evidence.

Since comprehensive programmes aimed to modify environmental determinants are considered most effective (WHO 'Gaining health strategy', 2006), the present paper illustrates a realist evaluation of a threeyears regional anti-doping programme ('Safe Gym - Prevention and wellness') aimed at modifying both cultural and environmental variables of the system sustaining the consumption of PES.

Objectives: a) Identifying social and organizational determinants of gym managers' positive orientation toward PES; b) identifying regulatory mechanisms and tools that may positively influence the development of a 'Safe-Gym' network, such as a Regional Plan for health promotion and an Ethic code that bans dietary supplements sale; c) evaluating motivations and expectation related to joining the 'Safe-Gym' network; d) evaluating the impact of the latter on gym managers' and attendees. A key point of the evaluation was to identify the expected and unintended mechanisms triggered by the programme and the facilitating conditions that could explain the voluntary 'loss of income' linked to the renounce to sale supplements representing a large percentage of many fitness and sport centres' turnover.

Method: The study used a pre-post evaluation design with a self administered questionnaires addressed to gym managers and sports coaches. A path-analysis was applied on pre-test data to verify the existence of meditational chains between subjective norms, beliefs and expectations of adherence to the programme and of impact on gym attendees. Also, case analysis with in depth interviews of stakeholders was carried out in three different municipalities to analyze the implementation process of the public-private partnership. Eighty-five managers and sport coaches, belonging to 59 gyms of different provinces, volunteered. The second part of the study, focusing on the ultimate target group of gym attendees, is still ongoing. It is aimed at evaluating the individual and potential interactive effects of receiving/not receiving an 'health promotion and informative tool' and/or attending/not attending gyms belonging to the 'Safe-Gym' ne! twork on attendees attitudes to the use of performanceenhancing substances.

Results: The theory of the programme and its main results and weaknesses are presented and the implications of shifting from 'command and control' measures to ban illicit substances toward a cooperative regulatory agreement between public administrations and civil society involved in sport and health policies are discussed. The paper concludes with suggestions for further methodological improvements for a theory-driven evaluation of complex programmes operating at different system levels and implying different chains of mediational mechanisms. 
Inside a new emerging social configuration: TV Audiences and Great Sporting Events (2000-2010)

\section{S. Martelli}

\section{Facoltà di Scienze Motorie, Università di Bologna "Alma Mater Studiorum", Italy}

In this paper I'll present some findings of my analysis on the data collected in Italy and some other countries from Auditel or other public companies, which every day -and every hour, and every minute...- measures the national TV audiences, by a very sofisticated tool -the meterpeople- in order to collect national audiometric data; their samples are a representative ones, indeed. So that everyone can know both the width and the social characteristics of the Italian audiences — or of the English ones, or Spanish ones, etc.- at a rough estimate.

The SportComLab of the University of Bologna "Alma Mater Studiorum" studied the Italian audiences, which from their own TV sets followed the Olympics, the Paralympics and the European and World Football Championships of the editions disputed in the period 20002010. My younger researcher and I studied the data collected by about 2.000 sport competitions, and made comparison using more than 13,8 millions of audiometric and socio-graphic data, in order to identify and to describe the tendencies in the observed period. Two research hypothesis -the «regional nexus» between a sport champion and his/her "mediated" fans, and the «sporting sub-cultures hypothesis» about the correlation between a kind of audience and its exposition to a sport program, and a set of social characteristics, which identify a sporting "niche"- led the comparison works. Two books of mine: Lo sport "mediato" [The "mediated" sport, FrancoAngeli, Milan 2010] and Lo sport globale [The global sport, FrancoAngeli, Milan 2012], collected the main bulk of these findings about the Italian Tv audiences of the great sporting events (2000-2010).

In my paper I'll introduce some remarks about the relevance for a social scientist to gain a better knowledge of the sporting audiences in his own country: indeed the TV audiences are the bulk of a new emerging social configuration, shaped by the relations and interrelations among three systems: the sport, the media, and the sponsors companies [n. 1]. Then I'll offer a selection of the main findings about width and social details of the Italian sporting audiences in the period 2000-2010: sometimes a huge public ( 25 millions and more of italian televiewers) saw the competitions of the main sporting events, as the matches between the Italian footbal team and other national representatives, as the team of France, Spain, Germany or Holland [n. 2]. Finally, I'll show two interesting exceptions to the "male reserve", which sport is at the TV public eyes in Italy: the Eris's effect produces an unusual crowding in front of the TV set of the females viewers, attracted by crucial moments of sporting competitions [n. 3]. These findings permit to prove that sport is a "total (and global) social phenomenum" (M. Mauss), indeed!

\section{Implicit and explicit anti-fat bias among university students}

\section{E. Gobbi, I. Ferri, A. Carraro}

\section{FISPPA Department, University of Padua, Italy}

Background: Anti-fat prejudice is widespread and pervades numerous areas of society including education, employment practice and medical health care and is a concern with considerable psychosocial costs. By using the Implicit Association Test (IAT; Greenwald et al., 1998) it has been found that health professionals and physical educators display significant implicit anti-fat attitudes. The purposes of this research was to examine the implicit and explicit attitudes toward obesity among a sample of university students, and to investigate the relationships with the psychosocial construct of the social dominance orientation.

Materials and method: Data were collected over two year period, undergraduate students of exercise sciences $(n=324)$, medicine $(n=314)$, and primary education $(\mathrm{n}=259)$ filled in a battery of questionnaires including demographic information. To assess implicit anti-fat attitudes it was used the IAT. The IAT is a timed assessment that measures automatic associations of a target construct (fat versus thin people) with particular attributes (lazy and stupid). Participants had $20 \mathrm{sec}$ to complete each of the IAT tasks: people generally find the tasks easier when the paired categories match their attitude, and they are able to correctly classify more words. The Crandall's Questionnaire was used to assess explicit attitudes, the Social Dominance Orientation scale (SDO) to assess social dominance orientation.

Results: Data with a IAT error rate higher than 35\% were excluded from analysis. The whole group reported significant levels of implicit anti-fat bias both for lazy $(\mathrm{t}(650)=21.77, \mathrm{p}<.01)$ and stupid $(\mathrm{t}(824)=$ $11.00, \mathrm{p}<.01)$ attributes. Positive significant associations were found for implicit anti-fat bias and the subscale "dislike fat" of explicit prejudice $(p<.001)$. Differences for gender were significant both for implicit $(p<$ $.05)$ and explicit anti-fat bias $(\mathrm{p}<.001)$, men reported higher values with the exception of "fear of fat" Crandall's subscale.

One-way ANOVA showed that undergraduate students in medicine reported significantly higher implicit anti-fat attitudes in comparison with other university students $(\mathrm{F}(2,648)=6.35, \mathrm{p}<.01$ for "fat-lazy" prejudice and $\mathrm{F}(2,822)=5.625, \mathrm{p}<.01$ for "fat-stupid"). Explicit anti-fat bias was higher among exercise sciences students for all subscales $(\mathrm{F}(2,894)$ $=28.525, \mathrm{p}<.01$ for "dislike fat"; $\mathrm{F}(2,894)=3.166, \mathrm{p}<.05$ for "fear of fat"; $F(2,894)=38.592, p<.01$ for "willpower" subscale). Moreover, high level of explicit bias were associated with higher SDO $(\mathrm{p}<.001)$.

Discussion: Medicine students displayed stronger implicit anti-fat bias, while exercise sciences students reported higher explicit bias and SDO. These results could have important implications for health promotion as may contribute to reduce quality of life for many overweight and obese people. Intervention strategies are needed to reduce these negative and potentially dangerous attitudes.

\section{Sport for the formation of the person: "sports as a tool for education, training of adults and children: a resource for school and strategy for the job"}

\section{F. Perrotta, A. Pannelli}

\section{Dipartimento di Scienze della Formazione, Università di Macerata,} Italy

Sport is synonymous with competition, conflict, rivalry, proactive and challenge to the last breath with the dominance of its competitive dimension, more crucial to enhance the values related more to the fight and the contrast between the contenders that all 'meeting, the comparison and the discovery of self and other. Meeting, discussion and discovery that are the foundation for growth and training of the person, a pedagogy that can guide the subject for the construction and sharing of basic rules for its social life, to discover and experience the values of tolerance, loyalty, solidarity, brotherhood and belonging.

A purpose and an educational process, two concepts that travel in harmony with each other, lead us to understand how the body-movementsports promote self-knowledge, the environment and its possibilities of movement and also contribute to the formation of personality through the pupil's knowledge and awareness of the body's identity.

In the common meaning today mean by sport physical activity which is carried on at least three different levels of emotional investment: for competition, for fun, wholesome activities like. These levels are anthro- 
pologically and psychologically acceptable if they are found together and integrated with each other, without a reason to prevail over the other.

In particular, we intend to do the speech, a redefinition is necessary precisely because we want marcarne the meanings of education and training, being men, that sport can still deliver even if, in my opinion, these meanings are covered in masks and modified pollutants from many influences.

Sport, for us, is like a vehicle for growth. The use of sports metaphors for svuluppo a team and the individual. Sport as a metaphor for shared challenge and tension to the success. The shared objective, the importance of the role, respect and the ability 'to manage the skills that are here now training alongside a sports team and make a unique team.

\section{ORAL PRESENTATIONS}

\section{Saturday October $6^{\text {th }}, 2012$}

\section{Session 6. Sport Technology and Materials - Functional Adaptation to Exercise}

\author{
Thermal aspect of (sports) clothing
}

G. Havenith

Environmental Ergonomics Research Centre, Loughborough University, $U K$

This paper will provide an overview of research performed in our laboratory that has direct applications in the design and evaluation of sports clothing and equipment. Mostly it focuses on more encapsulating clothing types, like mountaineering clothing and outdoor clothing in general, but the knowledge also provides input to athletic clothing design. Most recently the research has focussed on various aspects of body mapping, charting the human body in terms of heat production and cooling zones. This combined with extensive work on body sweat production mapping, has allowed manufacturers to create clothing which shows regional variation in its properties, supporting the physiological responses. Initially maps were obtained for the torso (running shirt design), hands (racket design) and feet (shoe/socks), developing a technique that allows sweat collection from large surfaces with minimal interference with the activity studied.

Another area of interest is that of heat transfer processes in clothing. It is generally assumed that sweat removal from the skin by wicking garments is beneficial. However, though this may be the case for comfort, recent work has shown that the cooling effectivity of such sweat may be reduced, and thus in events where heat loss is compromised such wicking may also have negative effects.

Techniques for collection of such data will be presented together with initial results.

Upper body high intensity interval training: effects on different components of physical fitness

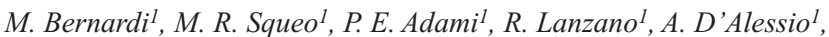
A. Rodio $^{2}$, L. Fattorini ${ }^{1}$

${ }^{1}$ Dept. of Physiology and Pharmacology, "V. Erspamer", University of Rome "Sapienza", Italy

${ }^{2}$ Dept. of Sport and Health Sciences, University of Cassino, Italy

Aim: We tested the hypothesis that a 6-7 week long (3 sessions per week) upper body (Ub) high intensity interval training (Ub-HI-IT) was effective in improving not only aerobic fitness, as previously demonstrated in endurance athletes, with training based on running (Saltin and Gollink, Handbook of Physiology - Skeletal Muscle, 555-631; 1983), and in active subjects, with series of 30 second long Wingate cyclo-ergometer exercises (Burgomaster et al., J. Physiol. 586, 1, 151-160; 2008), but also glycolitic capacity and body composition. The studied Ub-HI-IT was designed to be carried out at an intensity equal to $130 \%$ of the peak power reached in a previously performed maximal incremental aerobic arm cranking ergometer (ACE) exercise test and consisted of 5 bouts of ACE exercise, each one prolonged up to exhaustion, interspaced by a recovery time necessary to start each bout every 5 minutes.

Methods: Before and after the Ub-HI-IT, 11 moderately active healthy 
men $(37.1 \pm 10.72$ years, mass $77.1 \pm 9,13 \mathrm{~kg}$, height $1.79 \pm 0,063 \mathrm{~m})$ were submitted to an ACE maximal incremental exercise test in a standing posture to assess oxygen uptake peak (VO2peak) and the intensity ( $130 \%$ peak power) for both an ACE exhaustion test (Ex-T) and for the Ub-HIIT. The Ex-T was carried out to assess total mechanical work (TMW) and blood lactate (BL) peak. Before and after the Ub-HI-IT, also fat mass was measured through a whole body dual energy $\mathrm{X}$-ray absorption (DEXA) scan.

Results: Total mechanical work carried out during each training session (mean power: $215 \pm 23.9$ watt) changed from the initial values of $73.3 \pm 10.30$ to $135.4 \pm 19.32 \mathrm{~kJ}$ after $6.9 \pm 0.89$ weeks of Ub-HI-IT. VO2peak and TMW at the Ex-T increased after training from $37.1 \pm 7.35$ to $42.9 \pm 6.85 \mathrm{ml} \bullet \mathrm{kg}-1 \cdot \mathrm{min}-1(\mathrm{p}<0.001)$ and from $22.3 \pm 6.19$ to $33.1 \pm 6.87$ $\mathrm{kJ}(\mathrm{p}<0.001)$, respectively. The TMW improvement was paralleled by an increment of the energy expenditure of the Ex-T which changed from $159 \pm 7.01 \mathrm{~kJ}$ before training to $181 \pm 20.5 \mathrm{~kJ}$ after training with a BLpeak increase from $11.7 \pm 1.75$ to $13.3 \pm 2.77 \mathrm{mM}$. Fat mass expressed as percentage of body mass decreased from $18.2 \pm 3.09 \%$ to $16.5 \pm 3.87 \%$ $(\mathrm{p}<0.05)$ corresponding to a fat mass equal to $14.1 \pm 3.21 \mathrm{~kg}$ at the baseline and $12.1 \pm 3.15 \mathrm{~kg}$ after training.

Conclusions: A low volume Ub-HI-IT, with a single session lasting 21 minutes and with phases of actual work lasting $5.83 \pm 0.612$ and $10.60 \pm 1.938$ minutes in the first and in the last week of the training, respectively, was able to increase baseline Ub-VO2peak of $15.8 \pm 6.84 \%$ and baseline ACE TMW at the Ex-T of $46 \pm 10.7 \%$. The intensity of the exercise (VO2 reached values, as average of each bout and during the first week of training, equal to $90.2 \pm 6.72 \%$ of the baseline VO2peak and $\mathrm{BL}$ values were $19 \pm 0.2 \%$ higher than those measured at the Ex-T) and the consequent hormonal and cytokine responses to training are responsible of the improvements of aerobic and anaerobic fitness and of the better body composition.

\section{Differential effects of continuous $v s$ discontinuous aerobic exercise on oxygen uptake and vascular health}

\section{J. Landram ${ }^{\text {, A. C. Utter }}$, C. D. Curry ${ }^{2}$, J. L. Welsh', M. L. James ${ }^{2}$, S. R. McAnulty ${ }^{2}$, S. R. Collier $^{2}$ \\ IDept of Health Science, University of Rome "Foro Italico", Rome, Italy. \\ ${ }^{2}$ Dept of Health, Leisure, and Exercise Science, Appalachian State University, Boone, United States of America}

Background: Obesity and sedentary lifestyles have reached pandemic levels worldwide which over time leads to the progression of many chronic diseases or declining functional capacity. Aerobic exercise benefits both causal factors, with the current prescription of 30 minutes of moderate intensity exercise on most days of the week. Yet, few individuals can complete 30 continuous minutes and there is a paucity of literature investigating direct comparisons of continuous $v s$ discontinuous exercise.

Purpose: Therefore the purpose of this study was to examine the oxygen uptake and hemodynamic differences in continuous $v s$ discontinuous exercise in males and females.

Methods: Thirty six male and female subjects were recruited for this study. Subjects underwent a baseline test for VO2max and hemodynamic measurements. They were then assigned to a one month wait listed control period before returning to repeat the testing and to begin one month of either continuous aerobic treadmill work for 30 minutes at $70 \%$ max heart rate $(\mathrm{N}=18)$ or 3 bouts of 10 minutes of exercise at $70 \%$ of max heart rate with two 10 minute break periods in between, totaling 30 minutes of aerobic work (1:1, work:rest; $\mathrm{N}=18)$.

Results: Both continuous and discontinuous groups demonstrated a sig- nificant improvement in VO2max $(p<0.001)$, heart rate $\max (\mathrm{p}<0.05)$, augmentation index normalized for heart rate at 75 beats per minute $(p<0.01)$, central and peripheral pulse wave velocities $(p=0.05)$, and systolic and diastolic blood pressures $(\mathrm{p}<0.05)$. A treatment difference was detected between augmentation index normalized for heart rate at 75 beats per minute $(\mathrm{p}=0.021)$ with the discontinuous protocol yielding a greater reduction.

Conclusion: These data suggest that for healthy people either continuous or discontinuous aerobic training is effective in improving measures of fitness and vascular health, however in persons with arterial stiffness, discontinuous training may be more beneficial.

\section{Female skin conductance and regional differences in thermal sudomotor activity at rest and during exercise in different environments}

\section{Filingeri ${ }^{1}$, N. Gerrett ${ }^{1}$, S. Hodder ${ }^{1}$, B. Redortier ${ }^{2}$, G. Havenith ${ }^{1}$ \\ ${ }^{I}$ Environmental Ergonomics Research Centre, Loughborough University, Loughborough LE11 3TU, UK \\ ${ }^{2}$ Thermal Sciences Laboratory, Oxylane Research, Villeneuve d'Ascq 59665, France}

Introduction: Monitoring the skin conductance has been primary used within the psychological field as a method to estimate the level of tonic and phasic emotional arousal which has been shown to affect sudomotor activity. However, in the present work we explored the application of this method to the evaluation of regional variations in sudomotor activity at rest and during exercise in different environmental conditions.

Methods: Eight healthy university female students $(20.7 \pm 1.5$ years; $165.5 \pm 6 \mathrm{~cm} ; 60.4 \pm 7.9 \mathrm{~kg}$ ) volunteered to participate in the study. Each participant performed 4 trials in a climatic chamber in a balanced order. Participants entered the climatic chamber and $10 \mathrm{~min}$ were allowed to acclimate to environmental conditions. Then they were asked to rest on a chair (15 min) or cycling [ 5 min warm up ( 60 watts; $80 \mathrm{rpm}$ ) followed by 10 min exercise ( 60 watts; $40 \mathrm{rpm})]$ either in thermo neutral $\left(22^{\circ} \mathrm{C} ; 50 \%\right.$ $\mathrm{RH})$ or warm environmental conditions $\left(33^{\circ} \mathrm{C} ; 50 \% \mathrm{RH}\right)$. The MP-35 system (Biopac, USA) was used to monitor the electrodermal activity of 3 body regions (lateral upper and lower back and lateral abdomen). Local skin temperature was monitored at 5 body sites (iButtons, Maxim, USA) and mean skin temperature was calculated using Houdas-5 points formulas. The main effect of condition and time was tested using two-way repeated measures ANOVA. Post hoc comparisons were performed to analyse individual differences.

Results: Analysis of the data showed significant regional differences $(\mathrm{P}<0.05)$ in the skin conductance level during all the conditions. The lower back showed the highest absolute value $(11.4 \mu \mathrm{S})$ during exercise in the warm environment, followed by the upper back $(9.0 \mu \mathrm{S})$ and the abdomen $(8.3 \mu \mathrm{S})$. Mean skin temperature significantly increased $(\mathrm{P}<0.05)$ during the trials in the warm environment (rest: $33.6^{\circ} \mathrm{C}$; exercise: $33.7^{\circ} \mathrm{C}$ ) compared to ones performed in thermo neutral conditions ( $\mathrm{r}$ est: $31.4^{\circ} \mathrm{C}$; exercise: $31.05^{\circ} \mathrm{C}$ ) with no differences between rest and exercise $(\mathrm{P}>0.05)$.

Conclusion: The significant regional differences in skin conductance observed both at rest and during exercise seem to be aligned to the results of other studies which used more traditional methods (i.e. sweat pads, ventilated capsules) to investigate regional sweat rates. The lower back has been confirmed to play a primary role in contributing to the body's cooling power through a greater sudomotor activity than other regions during elevated thermal states. This novel approach provided useful information to indicate the evaluation of the skin conductance response as an effective method to investigate regional and intra-segmental sudomotor differences. 
Flight time is not enough when evaluating countermovement jump in old women

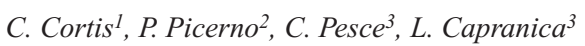

${ }^{1}$ Department of Human Sciences, Society and Health, University of Cassino e Lazio Meridionale, Cassino, Italy

${ }^{2}$ Sensorize Motion Sensing Technology, Rome, Italy

${ }^{3}$ Departement of Human Movement and Sports Sciences, University of Rome Foro Italico, Rome, Italy

Introduction: Association of stretch-shortening cycle activities, as performed in countermovement jumps (CMJ) are widely used for the assessment of muscle power in old individuals, being representative of functional ability, motor capacity and lower extremity neuromuscular function during independent daily living tasks (Spirduso et al., 2005). In field studies, CMJ measurements are usually performed by means of optical cells and contact mats. The estimate of jump height with those instruments relies on the assumption that the height of the center of mass at take-off and landing coincide, by asking the subject to land with lower limbs extended and to keep their trunk as vertical as possible from the starting position to the landing phase. However, no study investigates if these requirements are met in older individuals. Thus, the purpose of this study is to verify how CMJ performance parameters are related to jump execution in older individuals in field settings.

Methods: After a familiarization trial, 22 old women (age: $70 \pm 7$ years; body mass: $60.6 \pm 10.1 \mathrm{~kg}$; height: $160.1 \pm 6.1 \mathrm{~cm}$; BMI: $23.8 \pm 3.6$ ) were instructed to perform a fast downward movement immediately followed by a fast upward moment and to jump as high as possible keeping their hand on the hips. Instantaneous vertical acceleration, velocity and displacement of the centre of mass were estimated using a wireless 3D inertial measurement unit (FreePower, Sensorize, Italy) wore on the trunk at the level of L5 during the execution of each CMJ (Picerno et al., 2011). Jump height (as estimated using flight time), peak velocity (Vpeak), force (Fpeak), and power (Wpeak), the difference between the height of the center of mass at take-off and landing (DeltaHCM) and trunk bending (TB) were collected and submitted to a correlation analysis.

Results: Jump height $(10.9 \pm 3.5 \mathrm{~cm})$ was significantly $(\mathrm{p}<0.05)$ related with Vpeak $(\mathrm{r}=0.9 ; 1.3 \pm 0.2 \mathrm{~m} . \mathrm{s}-1)$, Wpeak $(\mathrm{r}=0.8 ; 19.2 \pm 4.2$ W.kg-1), DeltaHCM $(\mathrm{r}=0.8 ; 6.4 \pm 2.6 \mathrm{~cm})$, and TB $\left(\mathrm{r}=0.5 ; 26.4 \pm 11.7^{\circ}\right)$ while no

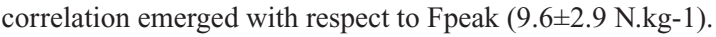

Conclusions: DeltaHCM value points out that the height of the center of mass at take-off and landing does not coincide, indicating that knee are flexed at the instant of landing, thus leading to an increase of the flight time and consequently to the artificial overestimation of jump height. TB values recorded in the present study are lower $\left(-10^{\circ}\right)$ than those reported for young subjects (Picerno et al., 2011), suggesting that older women tend to rely less on trunk rotation, which is responsible for $44 \%$ of CMJ performance (Luthanen and Komi, 1978). The results of the present study show that jump performance parameters estimated using solely flight time might be not sufficient when testing older individuals, thus indicating the need of performance parameters not only related to flight time.

References

- Luthanen and Komi (1978) EJAP 38:181-188

- Picerno et al. (2011) JSS 29:139-146

- Spirduso et al. (2005) Human Kinetics
Aerobic versus resistance exercise, cardiovascular adaptations in young and elderly female subjects

\section{A. Baraldo, C. Tarperi, A. Cevese}

\section{Dip. Sci. NNMM, University of Verona, Italy}

Purpose: A comparison of cardiovascular adaptations to resistance (RE) and aerobic (AE) exercise in Elderly (EF) and Young Females (YF) is an important and not yet completely understood topic in Sports Science. The purpose of this work was to delineate and quantify changes in the cardiovascular system during $\mathrm{AE}$ and RE bouts in $12 \mathrm{YF}$ $(23.7 \pm 2.5 \mathrm{yy}, 56.0 \pm 5.7 \mathrm{~kg}, 164 \pm 1 \mathrm{~cm})$ and $12 \mathrm{EF}(67.1 \pm 7.1 \mathrm{yy}$, $58.2 \pm 5.0 \mathrm{~kg}, 157 \pm 1 \mathrm{~cm})$.

Method: RE was performed on a leg press machine with three series (10' recovery) of 12 repetitions at $70 \%$ of individual $1 \mathrm{RM}$ (previously assessed by the Brzycki method). AR was performed on a cycle ergometer at constant load (80\% V'O2max), at 60-70 rpm for $5 \mathrm{~min}$ and repeated 3 times, separated by 10 min recovery. During both tests, we continuously recorded the finger arterial pressure by photoplethysmography (portapres, TNO) and calculated heart rate (HR), cardiac output (CO), stroke volume (SV), and total peripheral resistance (TPR).

Results: arterial pressure values increased to the same extent in YF and $\mathrm{EF}$, but the increase was greater in RE and was entirely due to changes in diastolic pressure. Heart rate increased more in YF than in EF, but the increase was unrelated to the exercise type. CO increased by $110 \%$ and $60 \%$ in $\mathrm{YF}$ and by $75 \%$ and $22 \%$ in $\mathrm{EF}$, for $\mathrm{AE}$ and $\mathrm{RE}$, respectively. These changes were caused by progressively decreasing vasodilatation, which turned into vasoconstriction in EF_RE. Changes in TPR lead to corresponding changes in SV that increased by $40 \%$ in YF_AR and decreased by $10 \%$ in EF_RE.

Conclusion: we conclude that age and exercise type affect the measured cardiovascular variables with different mechanisms. Resistance exercise increases diastolic pressure while age blunts the increase in heart rate. In addition, the relative influence of metabolic vasodilatation and mechanical hindrance on muscle blood flow, leading either to fall or to increase in TPR, depends both on exercise type and age.

Every resistance trainer, in particular with elderly females, should be considered that the efforts contained in this kind of exercise can represent a strong limitation to cardiac output increase. This can be a problem but also a useful cardiovascular training strategy.

\section{Can Wii modulate visuospatial attention lateralization?}

\section{G. Giglia' I, I, Gueli ${ }^{1}$, F. Brighina ${ }^{1}$, D. Zangla 2 , A. Bianco ${ }^{2}$, A. Palma ${ }^{2}$, B. Fierro $^{l}$}

${ }^{1}$ Department of Experimental Biomedicine and Clinical Neuroscience (BioNeC) Via G. La Loggia, 190100 University of Palermo, Italy ${ }^{2}$ Department of Sports Science (DISMOT) University of Palermo, Italy

Objectives: In a previous study we found that "open-skill" high level athletes (volleyball players) performing a line-length judgment task responded significantly faster and were also more accurate, as compared with closed skill athletes (rowers) and controls. As the design of the study did not allow to know if such results were due to training rather than self-selection of ability, in the present study, differences in visu- 
ospatial attention lateralization were investigated in healthy volunteers before and after a short period of virtual "open skill" sport playing. Materials: Subjects: 18 sedentary participants.

Experimental procedure: Computerized line length-judgement task using Psyscope X software on a portable apple computer 13".Virtual open skill sport (tennis) on an active system game consolle, the "Nintendo Wii", and the "Wii Sports games" software "tennis". The controller used was a "Wii remote", a wireless 3-axis accelerometer that works with gesture recognition. Method: Subjects responded to a computerized line-length judgment task, before and after a short period (7 days, 1 hour/day) of virtual "open skill" sport playng (tennis). Five lines, differing in the length of their right and left segments, were randomly presented; the respondent made a forced-choice decision about the respective length of the two segments. Reaction times (RTs) and percentage of errors towards left and right were recorded and analized by means of ANOVA

Results: In baseline condition subjects showed a tendency to bisect lines slightly to the left, a phenomenon known as pseudonenglect. The oneway ANOVA for the percentage of errors Pre $v s$ Post-Wii showed a significant main effect, with a significant reduction in the post-wii condition $(\mathrm{p}<0.05)$. The two-way ANOVA comparing leftward versus rightward errors between conditions: pre- vs Post-wii, with Errors (leftward and rightward) and Condition (pre- $v$ s Post-wii) as within subjects factors showed a statistically significant main effect $(\mathrm{p}<0.05)$. Duncan's post hoc analysis showed that in the Post-wii condition, subjects made statistically significantly fewer errors to the left compared to the pre-wii condition. The one way ANOVA for RTs showed no significant differences between conditions.

Discussion: Visuospatial attention is a cognitive function considered to represent an important determinant of success in open skill sports. In such sports, the environment is constantly changing and movements have to be continually adapted. The ability to process spatial information and perceive differences or asymmetries correctly in both hemi-visual fields allows athletes to perform successfully. The main result of the study was that a short period of virtual open skill sport playing was able to counteract the physiological hemispheric imbalance, improving visuospatial attention isotropy.

Conclusions: The results may suggest the possibility of changing the distribution of visuospatial attention by virtual training.

\section{Session 7. Law, Economic and Social aspects of Sport and Fitness activities}

\section{Sports law and minors' participation}

\section{Santoro, S. Rigazio}

DISMOT, Dipartimento Studi Giuridici, Economici, Biomedici, Psicosociopedagogici delle Scienze Motorie Sportive, Università degli Studi di Palermo, Italia

The topic related to the participation of minors (people under 18 years old) in sport activities represents a particular and critical theme among the sport legal system. The civil code rules in respect of legal capacity are indeed insufficient and inadequate to assist underage athletes, specifically if they are engaged in professional sport activities that require a great amount of energies and time and that involve important economic resources (i.e. sponsors contracts...).

The sport system, then, offers the chance to witness and make observations regarding the complex world of minors specifically to who, among the experts, believe that it is necessary to go beyond the traditional doctrine in civil law, which tends to put the minor completely subject to his parents' willingness and, on the contrary, tend to evaluate the minor's actions in a modern perspective based on the recognition - with specific details - of his autonomy.

Following this last statement, the most recent and clear example is given by the story of Laura Dekker, the Dutch sailor who, at the age of sixteen, has set the record in solitary around the world. While she was considered "not capable" and consequently "not mature enough" by the legal system, she was clearly not only capable but fully mature to undertake such an adventure from the sport perspective.

While it is true that the law assign to the parents the power of representation in all their sons' acts, it is also true that the "responsibility" towards them, according to article 147 of the civil code, means that they should help and assist their sons in order to make them choose what they "feel" it is right for them and not, as the traditional doctrine embraces, impose their own decisions.

Even if this is a relatively recent school of thought, it is possible to see some of this orientation, whose central element is the recognition of the "ability to discern" of the minor, on a national basis (labor law contract for 16 year old, autonomy of the student in choosing to attend Catholic religion class...) as well as on a international basis (specifically with the approval of the Convention on the rights of the child - CRC).

The demanding task for the scholars is then finding the balance between the necessity to respect the role of the parents (according to the civil code provisions) and the necessity to respect and promote the gradual acquisition of autonomy of the minor, always taking in consideration the fact that the key to this equilibrium is the acceptance of a dynamic perspective where the minor is first of all a person with a whole series of rights and not inferior to an adult.

\section{Related party transactions in Italian professional football clubs: an empirical study}

\section{S. Cincimino}

\section{Dept. DISMOT, University of Palermo, Italy}

Related party transactions assume an important role in the financial statements of limited companies, because they ensure the transparency and the substantial accuracy of the information.

In Italy, detailed information on related party transactions are scheduled by 2004 only for a limited class of companies, and only since 2009 for all the limited companies.

The professional football clubs are not exempt from this requirement. First of all, because in Italy the teams must be limited companies for participating in a professional competition.

Moreover, related party transactions are numerous and for different reasons, with inevitable effects, even distortive, on the representation of the economic and financial performances of the clubs.

For these reasons, in the recent edition of the club licensing manual, UEFA has planned for each club that wants to participate in European competitions, the clarification of specific information on related parties. Moreover, for the respect of the break-even result, useful for the purposes of the financial fair play, the club must determine the fair value of any related party transaction. Moreover regarding the incomes, no upward adjustments can be made. Thus, "if the estimated fair value is different to the recorded value, the relevant income must be adjusted". Regarding the expenses, no downward adjustments can be made. So, "if the estimated fair value is different to the recorded value, the relevant expenses must be adjusted".

The paper presents the first results of a research that has for object the qualitative and quantitative analysis of related party transactions of some 
Italian professional football clubs.

In qualitative terms, we investigate on the exhaustiveness of the information, and on its contribution to the transparency and the substantial accuracy of the financial statements of clubs.

In quantitative terms, we analyze the financial statements of the clubs and the impact of related party transactions on their economic and financial situations, with useful simulations for verifying the possible gap compared to the parameters of financial fair play.

As expected results, the paper aims to highlight the "weight" of related party transactions in the professional clubs, the quality of their representation in the financial statements and the impact on economic and financial performance.

The methodological approach of this paper is the case analysis. Carrying out a research through case analysis is, in fact, like performing laboratory experiments (Yin, 1994). We chose the so-called purposive sampling, often used to obtain illustrative outlines of specific realities through the choice of particularly representative cases.

\section{Pluralism of the professional sport figures}

\section{F. Blando}

\section{DISMOT, University of Palermo, Italy}

The present work regards the world of professions in sport. Legally speaking, there is no exact rule so that juridical relativism reigns. Indeed, on the one hand we find the only indication in the law n.91 of 1981, while the general law ignores completely the topic; on the other hand we have some examples from other legal systems such as, for example, the sport system itself and the regional system.

Based on these data, we can easily affirm that the "pluralism of the professional sport figures" is the result of different factors found between the general legal system (i.e. the State law) and other particular legal systems. Regarding these last ones, the sport legal system plays a specific role, since the different kind of professionals present in it reveals a real autonomous legal system.

Also, the regional legal system is characterized by a strong presence of rules in this regard. The DPR 616/77 is the first of a long series of laws, all related to the regional functions or found among the competence about "professionals figures".

Though, the most influential intervention in the subject is represented by the constitutional reform in 2001 with the introduction of the "sport legal system" among the "concurrent competence" between the state and the regions (art.117, comma 3, Cost). This specific division of competencies is particularly interesting from a legal point of view since it mentions specifically the topic of professions, so that, theoretically, regions could step into the ruling of this subject, allowed by a constitutional provision.

The Constitutional Court has indeed intervened with two critic decisions on the matter of the division of competencies between the central state and the regions. In particular, with the decision n. 230 in 2011, the Court has held unconstitutional a regional law, which contained a specific discipline on sport professions and the consequent creation of specific directories because the Court claimed that it is the State's role to identify the different professionals figures. This decision, then, means that the topic of sport professions appears to be a pure State matter while, on the contrary, the regional contributions in this matter has always been critical and efficient to the improvement of the sport sector.

\section{The "leaky pipeline" in academic careers of Italian movement science faculties}

\author{
F. Guidotti ${ }^{1}$, P. Impedovo ${ }^{1}$, C. Minganti ${ }^{2}$, C. Cortis, ${ }^{3}$ M. F. Piacentini ${ }^{I}$, \\ A. Tessitore ${ }^{I}$, L. Capranica ${ }^{I}$ \\ ù \\ ${ }^{1}$ Department of Human Movement and Sport Science, University \\ of Rome Foro Italico, Rome, Italy; \\ ${ }^{2}$ Department of Clinical and Experimental Medicine, University \\ Magna Graecia, Catanzaro, Italy; \\ ${ }^{3}$ Department of Sport and Health Sciences. University of Cassino \\ and Southern Lazio, Cassino, Italy
}

A strong hegemonic masculinity is present in leadership positions, especially evident in academia (European Commission, 2009) and in sport (IWG, 2012), with women representing only $15 \%$ of full professors and $10 \%$ of board directors of sport organizations. Thus, the aim of this study was to verify gender equality in Italian Sport Science academic positions (i.e., researcher, associate professors and professor).

Methods: The study was conducted from September to December 2011. Gender distribution of academic positions (i.e., researcher, associate professor and professor) in sport sciences (i.e., M-EDF/01 and MEDF/02) and in 9 Movement Science Faculties (Bologna, Cassino, Chieti-Pescara, Rome Foro Italico, Milan, Naples Parthenope, Palermo, and Verona) was extrapolated by the National database. Furthermore, a 20 -items semi-structured on-line questionnaire was created to verify gender differences in career progression, including 15 closed-ended items, 5 open-response items, and 3 comments to closed-ended items. Three-hundred-twenty-four members of the 9 Movement Science Faculties were invited to fill the on-line questionnaire. Chi-square and MANOVA were applied to verify gender differences $(p<0.05)$ for academic positions and individual responses, respectively.

Results: Differences ( $p=0.004$ ) for gender emerged only between academic positions of Movement Science Faculties ( $\mathrm{n}=324$; professors: $\mathrm{m}=72 \%, \mathrm{f}=28 \%$; associate professors: $\mathrm{m}=66 \%, \mathrm{f}=34 \%$; researchers: $\mathrm{m}=51 \%, \mathrm{f}=49 \%$ ), despite a similar trend was observed also for M-EDF $(\mathrm{n}=157$; professors: $\mathrm{m}=74 \%, \mathrm{f}=26 \%$; associate professors: $\mathrm{m}=68 \%$, $\mathrm{f}=32 \%$; researchers: $\mathrm{m}=58 \%, \mathrm{f}=42 \%$ ). Response rate of the questionnaire was $25 \%(\mathrm{~m}=29, \mathrm{f}=52$; professors $=13$, associate professors $=23$, researchers $=23$ ). With respect to men, women perceived to be less esteemed $(\mathrm{p}=0.016, \mathrm{~F}=3.8 \pm 0.9, \mathrm{M}=4.5 \pm 0.6$ ), a lack of attention to their needs to combine family and work duties $(\mathrm{p}=0.026, \mathrm{~F}=3.3 \pm 1$, $\mathrm{M}=4.1 \pm 1.2$ ) and a lack of equal opportunities in career progression $(\mathrm{p}=0.007, \mathrm{~F}=2.9 \pm 1.2, \mathrm{M}=3.9 \pm 1.2)$. In particular, lack of time, difficulties in managing their family role, lack of flexibility in working schedule, and a persistent male hegemony were mentioned as critical barriers to female academic career progression.

Discussion: These results confirm previous studies on perceived gender inequalities and critical obstacles for women's progression in academic career (Etzkowitz et al., 1994). In considering the similar proportion of male and female researchers, there is a need to monitor the progression of academic careers in relation to gender.

\section{References}

- European Commission (2009) Women and Science teh March Towards Equality. Research.eu Publ.

- International working Group on women and Sport (2012) The Sydney Scoreboard. http://www.sydneyscoreboard.com/global-scoreboard/

- Etzkowitz H. et al (1994). The paradox of critical mass for women in science. Science: 266: 5182: 51-54. 
Subnational policies for sports and motor activities: a comparison between Sicily and Emilia Romagna

\section{A. La Spina ${ }^{1}$, A. Scaglione ${ }^{2}$}

IProfessore ordinario di Sociologia, Dipartimento Studi su Politica, Diritto e Società "G. Mosca",

${ }^{2}$ Assegnista di ricerca in Sociologia, Dipartimento Studi su Politica, Diritto e Società "G. Mosca"

The Italian Legislative decree n. 150 of 2009 has set the criteria to measure and evaluate performance. In theory, it is clearly a huge shift in perspective, both from a formal and substantial point of view. Public administrations now have a duty to improve the quality of the services offered through their public policies "put in place to satisfy the eventual needs of the community". In other words, alongside a clear and precise definition of the objectives they wish to meet, the monitoring of activities and the improvement of benefits accruing to the people and transparency, the institutions are therefore required to divulge the attainment of these results on the basis of the actual satisfaction of stakeholders, among which are, primarily, citizens.

As far as the promotional interventions of sports and motor activities are concerned, it may be interesting to look at the results of sub-national public policies, through a comparative analysis and, by using the approach of public policies evaluation, of two distinct cases of policy tried by Italian regions: the first, implemented in Sicily, and the second in Emilia Romagna. The choice of these two regions is not by chance. Sicily suffers from a chronic delay in comparison to other Italian regions, both with regards to the offer in the field of sport and also the demand. The regional administration of Sicily has not been able to stimulate and encourage the practice of sport on the island, in fact their best cases have been the odd, single initiatives without any longterm planning. On the other hand Emilia Romagna is one of the topranked regions in the national statistics both for the availability of sports facilities and structures and also for the actual practise of sports. Even the regional administration has shown an acute awareness to this issue, and has reacted by introducing solid and sustainable sports policies.

The contribution which we propose, intends to highlight, after research has been made into the accurate situation in the mentioned regions, the strengths and weaknesses of the policies for sports and motor activities, especially within the context of planning and setting up initiatives, and in addition, pointing out further ideas to improve the existing initiatives.

As obvious as it may seem, we believe that an ex-ante evaluation with the aim of putting in place a correct sports policy agenda represents a decisive step to attain the stated objectives and to ensure the success of the policy itself.

The quality of the educators as a key factor of the organizational success: the case of the "Masi" Sport Club

\section{A. Borgogni, S. Digennaro, E. Vannini}

Department of Human, Social and Health Sciences, University of Cassino and Southern Lazio, Italy

The "Polisportiva Giovanni Masi" has been studied by our research group since the year 2010/11. The club is based in Casalecchio di Reno, a municipality of 38000 inhabitants bordering Bologna: it has more than 7.000 members, and a large array of services and activities implemented, resulting the leading organization for the local community.
The research has been carried out with a special focus on the quality of the services provided, the main features of the general structure and the organizational processes, the interaction with public and private stakeholders, the level of satisfaction of the members, and the effects of changes occurring within the Italian sport system.

The research, ideographic in nature, has been carried out within the case-study approach. Particularly, questionnaires with members $(n=1100)$, in-depth interviews $(n=11)$, focus groups $(n=9)$, life histories $(n=4)$ and observations have been interpolated. Results of the study show that Polisportiva Masi is recognized as a high quality organization both in terms of services for the member and in terms of organizational processes that are now undergoing a phase of sensitive development.

The current follow-up study, based on a workshop and on three focus groups, highlights that several organizational processes have started, in a measure due to the results of the research.

The main factors of the high degree of the members' satisfaction for the provided services (very good $=12,7 \%$, good $=77,6 \%$ of the respondent) are the relationship with the sports educators/coaches/instructors (very satisfied $=58,9 \%$, satisfied $=27,8 \%$ ) and the assessment of their professional competences (very good $=43,4 \%$, good $=40,7 \%$ ).

These data, which highlight as the success of the organization is, above all, linked to the quality of the didactics, are deeply rooted in the history of the Sports Club.

Masi, in fact, has been within the first associations to propose psychomotricity classes in 1975 and formation courses with A. Lapierre in 1980, as well as baby swimming; rendered the formation mandatory in 1986/87; has invested in high quality formation throughout courses and cultural events; has organized volunteering community based educational and social services; is provided with an educational internal agreement; is strongly committed in maintaining and updating the founding values.

These are the background elements leading to the high level of members' satisfaction towards the educators, 50\% Physical Education or Sport Sciences graduated, and, through them, towards the entire organization.

Our research proves that, in the investigated case, the valorisation and actualization of the history and the investment in formation are key determinants of the organizational success.

\section{Session 8. Sport Technology and Materials - Training \& Performance}

\section{sEMG assessment of upper limb muscles during dynamical contractions in different instability conditions}

\section{E. Tomasoni ${ }^{1}$, M. Romanazzi ${ }^{1}$, G. Boccia ${ }^{2}$, A. Rainoldi $^{2}$}

${ }^{1}$ School of Exercise \& Sport Sciences, University of Turin, Italy ${ }^{2}$ Motor Science Research Center, School of Exercise \& Sport Sciences, University of Turin, Italy

Introduction: The influence of unstable surface on muscle activation during resistance training has recently been studied in literature. The role of different types of instability on muscle activation during bench press exercise or using barbell or dumbbell are still unclear. The aims of the study were to assess neuromuscular activation of the muscles involved in the extensive kinetic chain of upper limbs in exercises characterized by different stability conditions and load types. 
Metodhs: Eighteen resistance-trained subjects (age $25 \pm 5$ years; height $174 \pm 5 \mathrm{~cm}$; weight $76 \pm 8 \mathrm{~kg}$ ) were evaluated performing six different exercises in randomized order: two barbell exercises (flat bench press BP) and Smith Machine bench press SMBP) and four dumbbell exercises (dumbbell bench press DBP, dumbbell incline bench press DIBP, flat dumbbell fly FDF, dumbbell bench press on swiss-ball BPSB). Load intensity was set at $8 \mathrm{RM}$. sEMG signals were recorded using multi-channel electrodes arrays from six muscles: sternal portion of the pectoralis major (PM), anterior deltoid (AD), long head and lateral head of triceps brachialis (long_TB and lateral_TB), upper trapezius (UT), and serratus anterior (SA) muscles. Average rectified value (ARV) of sEMG signal was estimated. Friedman tests were used to assess the difference of each muscle activation among different exercises.

Results: ARVs of PM, long_TB and lateral_TB, were found greater in SMBP and BP $(p<0.05)$ with respect to the other exercises. ARVs of $\mathrm{AD}$ and SA were found lower in FDF with respect to the other exercises $(p<0.05)$. ARVs of UT were found greater in DIBP with respect to the other exercises $(\mathrm{p}<0.05)$.

Discussion: The activation of PM, long_TB, and lateral_TB were found greater in barbell exercises (SMBP and BP) with respect to dumbbell ones. This finding showed that more constrained exercises generated a greater muscle activation of prime movers in bench press. The monoarticular exercise (FDF) showed the lowest activation of two synergistic muscles, SA and AD. The lower level of coordination needed in this condition seemed to decrease synergistic muscles activation. The inclination of the body seemed to influence only the activation of UT that was greater in DIBP with respect to other conditions.

\section{Neuromuscular activation during isometric contraction in the absence of a visual feedback}

\section{E. Limonta ${ }^{1}, E . C \grave{e}^{l}$, S. Rampichini ${ }^{1}$, F. Esposito $^{l}$}

${ }^{1}$ Department of Biomedical Sciences for Health, University of Milan, Milan, Italy

Visual feedback is an essential component when attempting to minimize force fluctuations and to hold force steady. The aim of the study was to evaluate the force output control in the complete absence of a visual feedback. The presence of a learning effect due to repeated contractions without visual feedback was also assessed. After determination of the maximal voluntary contraction (MVC) of the knee extensor muscles, ten physically active males (age $23 \pm 1$ years; stature $1.74 \pm$ $0.07 \mathrm{~m}$; body mass $71 \pm 6 \mathrm{~kg}$; mean \pm standard deviation, SD) performed isometric tasks at $20 \%, 40 \%$ and $60 \% \mathrm{MVC}$ for $20 \mathrm{~s}$, in a random order. For each intensity, a trial with force visual feedback (FB) was followed by 3 trials without visual feedback (noFB-1, noFB-2 and noFB-3). During contraction, force output and electromyographic (EMG) signal from the vastus lateralis muscle were recorded. From the force signal, the coefficient of variation $(\mathrm{CV}$, an index of force stability), the distance of force signal from the required target ( $F$, an index of force accuracy) and the time within the target (t-target) were then calculated. From the EMG signal, the root mean square (RMS) and the mean frequency (MF) of the power spectral distribution were calculated. MVC was $69.3 \pm 3.9 \mathrm{~kg}$. In noFB-1, while CV was similar to FB, $\mathrm{F}$ was significantly higher and $\mathrm{t}$-target significantly lower $(\mathrm{P}<0.05)$ than in FB. Moreover, in noFB-1 EMG-RMS was lower and EMG-MF higher $(\mathrm{P}<0.05)$ than in $\mathrm{FB}$. When comparing the three noFB trials, a decrease in $\mathrm{F}$ between noFB-1 and noFB-3 $(\mathrm{P}<0.05)$ and an increase in t-target from noFB-1 to noFB-3 $(\mathrm{P}<0.05)$ occurred at $20 \% \mathrm{MVC}$. On the contrary, no differences in EMG parameters were retrieved among noFB-1, noFB-2 and noFB-3. These findings suggest that visual feed- back did not affect force stability, but it increased significantly force accuracy. Moreover, the repetition of trials without visual feedback improved force accuracy at low exercise intensity, unvei ling a learning effect in this kind of motor task.

\section{Kinematic and elettromyographic differences between front and back load in a double poling ski ergometer}

\author{
L. Bortolan ${ }^{1,2}$, B. Pellegrini ${ }^{1,2}$, C. Zoppirolli ${ }^{1,2}$, F. Schena $^{1,2}$ \\ ${ }^{I}$ CeRiSM - Research Center "Mountain, Sport \& Health" - \\ Università \\ di Verona, Rovereto, Italia \\ ${ }^{2}$ Dipartimento di Scienze Neurologiche, Neurofisiologiche, \\ Morfologiche e Motorie - University of Verona, Italy
}

Introduction: The importance of double poling technique in classical cross country ski races has been underlined by a lot of authors. This technique involves massive use of upper body muscles (Holmberg, 2005 ) and requires specific force training sessions. To this aim different ergometers have been developed in the last few years. The aim of this preliminary study was to compare biomechanical and muscular differences between a standard pull-down ergometer (PDE) and a new idea of back-load ergometer (BLE), that would permit to better emulate double poling technique during maximal force training.

Methods: One subject performed, in the same session, nine repetitions using the PDE and the BLE (load: $85 \%$ of 1 RM previously defined). The force exerted through the arms, the range of movements and EMG amplitude ot the upper body and leg muscles (Triceps Brachii (TBr), Posterior Deltoid (PD), Pactoralis major (PM), Latissimus Dorsi (LT), Rectus Abdomini (RA), Obliquus Externus (OBLe), Rectus Femoris (RF), Biceps Femoris (BF), Tensor Fascia Latae (TFL) and Vastus Lateralis (VL)) were measured. The differences between the ergometers for all parameters were evaluated by a paired t-test. The statistical level of significance was set at $\mathrm{p}=0.05$.

Results: The force exerted through the arms were not different between PDE and BLE whereas BLE poling time was $70 \%$ higher. The initial direction of force exertion was comparable between the ergometers while the minimal inclination was higher for BLE (PDE: $49.6^{\circ} \pm 3.4$; BLE: $34.6^{\circ} \pm 2.9$ ). The angle between the line from tip of the foot to the shoulder and the floor (body inclination) did not change during the poling action for PDE (initial: $87.8^{\circ} \pm 1.3$; final: $88.6^{\circ} \pm 1.0$ ) whereas for BLE ranged from $57.3^{\circ} \pm 3.5$ to $86.1^{\circ} \pm 2.4$. RMS values showed an higher activation for BLE in abdominal and leg muscles (RMS\%PDE: RA:+107.5; OBLe:+121.7; RF:+77.7; BF:+60.2; TFL:+99.4; VL:74.1).

Discussion and conclusion: No differences in force exertion between the standard and the new ergometer have been found. Shoulders and arms EMG activity doesn't change between the two ergometers, however in the use of the new back-load ergometer an increase in the activity of abdominal and leg muscles was seen and it was referred to the need to recovery the legs and to reduce BLE inclination during the poling. Moreover the position of the skier during the action seems to be more similar to modern double poling technique. Nevertheless these were preliminary results, the new BLE ergometer seems to allows an higher specificity in the double poling force training. Further analysis must be conducted to check for the similarity of the biomechanics and muscular pattern between BLE and double poling skiing on the field.

\section{References}

- Holmberg, HC et al (2005) A Biomechanical Analysis of Double Poling in Elite Cross-Country Skiers. Med Sci Sports Exerc. May;37(5):807-18 


\section{A study of the position's effect on the three-points 'shooting biomechanical posture in basketball players}

\author{
F. Sgrò ${ }^{1}$, S. Pignato $^{1}$, R. Lanzerotti ${ }^{1}$, P. Mango ${ }^{1}$, M. Lipoma $^{1}$
}

${ }^{I}$ Engineering, Architecture and Physical Activities Faculty, University of Enna, Enna, Italy

Introduction: Several authors addressed the biomechanical aspects of the basketball shooting, studying the effects of position of shoot, rule, and condition of players (Miller\&Bartlett, 1996; Uygur, 2010). Everyone agrees that one of the most important elements in shoot effectiveness is the release height. In this work we present a preliminary study on kinematics adjustment of three-point shooting in different positions and conditions.

Methods: Three healthy male guards, right-handed, (age:28,8 \pm 5 ; height:1,85 $\pm 5 \mathrm{~cm}$; weight: $76,3 \pm 6 \mathrm{~kg}$ ), with about ten years of experience, were examined using a hybrid markers-based video-analysis approach, using two cameras, one on sagittal plane and the other on frontal one. The markers were placed unilaterally in order to define a four-point biomechanical model of low-leg and three-point biomechanical model of upper-leg, that describes two and one relative angles respectively. The shoots were performed in three different positions outside of the area of basket, (centre, middle and corner) and in three different physical-stress conditions (rest without opposition, after training-to-fatigue program with and without opposition). We analysed five successful shoots for each athletes, position and condition that, as suggested in previous works (Rojast et al, 2000), doesn't touch the rim or the backboard. A score analysis was also conducted examining the number of successful shoots on the total ones according to athletes, condition and position.

Results: Data reported are relative knee and elbow angles, in two different phases of shoot, maximum loading phase and release one, and the height of release, and they are examined in the three different position. A preliminary Kolmogorov-Smirnov normality test was conducted and only the relative angle values in release phase doesn't follow a normal distribution. Later we applied a Pearson and Spearman correlation test and a one-way ANOVA test, with position as independent variables and a post-hoc Bonferroni test, setting significance level to $p<0.001$ and $\mathrm{p}<0.005$ respectively.

Discussion: Results of correlation test showed that the knee angle in maximum loading position had positive correlation with the height of release $(r=0,40 \mathrm{p}<0.001)$, while no significant correlation was found among the others biomechanical index. These sentences could suggest that less loading angle improve the height of release. Furthermore, we studied the relationship between the height of release and the position with an ANOVA test confirming, as previously established also with the score analysis, that a kinematics adjustment is necessary changing the shoot position.

References

- Miller S.\& Bartlett R., Journal of Sport Sciences, 14, pp.243-253, 1996

- Rojas et al., Ergonomics, 43, pp. 1651-1660, 2000

- Uygur et al., Journal of Human Kinetics, 24, pp. 51-56, 201

\section{A proposal of a match-analysis evaluation method for the offensive} game construction in youth soccer

\section{A. Ciuffarella, N. Bovino, L. Russo}

Dept. Biomedical Sciences and Technologies, Motor Sciences Faculty, University of L'Aquila, Italy

Introduction: The aim of the study is to suggest a method used by us with a young soccer team (U16). The focus of the method is the evalua- tion of offensive game construction through notational analysis $(1,2)$. We analyzed short and long passages, tackles, fouls and shots.

Methods: 9 matches were analyzed with an Event Recording Notational Analysis $(3,4)$ through the K-VideoScout software (K-Sport, Pesaro, Italy). The first match was considered as the baseline of our study, the following 8 matches were analyzed to monitor the effects of our training proposal. Some training proposal about the ball possession and the offensive game construction were performed during each training session, basing on the results of the first match analyzed. The observation process was reliable with an high ICC value (99\%)(5).

Results: The analyzed parameters showed an increasing trend during the training period. Short passages increased from $38 \%$ to $61 \%$; long passages decreased from $28 \%$ to $5 \%$; tackles from $22 \%$ to $15 \%$; fouls from $7 \%$ to $13 \%$; shots from $5 \%$ to $6 \%$.

Discussion: Data suggest that the training proposal was an effective one and players applied on the court the training principles. Data show an improvement in offensive game construction, principally for an increase of short passages $(+23 \%)$ and a decrease of long passages $(-23 \%)$, other data do not show any significant modification.

\section{References}

1. Rampini et al., 2007

2. Capranica et al., 2001

3. Carling, Williams \& Reilly, 1995

4. Darst, Zakrajsek \& Mancini, 1989

5. Hughes \& Franks, 2004

\section{Multimodal imaging techniques in sport}

V. Cavedon ${ }^{1,2,3}$, F. Piscitelli $^{1,2,3,}$ C. Lovato $^{2,3}$, C. Milanese $^{1,2}$, A. Giachetti $^{4,5}$, C. Zancanaro $^{1,2}$

${ }^{1}$ Faculty of Motor Sciences, University of Verona, Italy

${ }^{2}$ Department of Neurological, Neuropsychological, Morphological and Movement Sciences, University of Verona, Verona, Italy

${ }^{3}$ Doctoral program in Multimodal Imaging in Biomedicine, University of Verona, Italy.

${ }^{4}$ Faculty of Education Sciences, University of Verona, Italy

${ }^{5}$ Department of Computer Science, University of Verona, Italy

Anthropometry and body composition analysis have been largely applied in scientific research to identify different subgroups within a certain population. Recent progress in both fields is changing the way these study are performed and open a lot of new possibilities.

Body composition analysis can exploit imaging devices like dual-energy X-ray absorptiometry (DXA) that currently represents the gold standard to estimate fat mass, lean mass, and bone mineral content and new generations of acquisition devices like ultrasound scanners, air displacement plethysmographs, etc.

Anthropometry is then becoming fully digital: 3D scanners can now acquire very accurate models of human subjects, allowing automatic or computer assisted measurement of body lengths, breadths, diameters, volume and surface area.

Considering that current techniques used to estimate body surface area as well as total body or segmental volume are based on the use of formulae, it is easy to understand the potential improvements provided by digital models.

A relevant task for research in body shape and composition analysis is therefore related to the validation of digital measurements. In our work we developed computer tools to evaluate a variety of shape parameters from original noisy scanner models, trying to validate them following two main research directions.

The first consists of comparing body scanner data with other well-established biometric technologies. We therefore compared measurements 
obtained with standard tape-based procedures with similar ones simulated with a custom made software tool on the digital models, obtaining comparable results for most of the analyzed measures. Results indicate that digital anthropometry allows for accurate and reproducible measurements also when operators lack specific training.

The second research approach aims at developing a totally automatic analysis of body shape in order to obtain measurements from raw scanner data. Given the great amount of data made available by the body scanning technology, developing viable, automatic and robust procedures is essential. Computational geometry tools like remeshing and skeletonization algorithms, etc. have been applied to analyze the models and automatically extract from them shape parameters independently on pose variations and with sufficient robustness against acquisition noise or holes.

Being these parameters not directly comparable with tape-based methods, a possible validation approach we are testing is related to a direct correlation of the measurements with composition data acquired with the DXA scanner. In a preliminary study we found that several automatically extracted body measurements (obtained in the abdominal region) are highly correlated with trunk fat ( $r \geq 0.9$ with maximum Anterior-Posterior diameter, maximum Mean Diameter and maximum of slice Area) and total body fat ( $\mathrm{r}=0.76$ with maximum Anterior-Posterior diameter, $\mathrm{r}=0.89$ with maximum Mean Diameter, $\mathrm{r}=0.88$ with maximum of slice Area).

\section{Reliability and validity of the MarkWiiRTM for kinematic analysis}

S.Vando ${ }^{1}$, F.Pizzolato 2 , D.Bagno ${ }^{4}$, J. Padulo ${ }^{1,2,3}$

${ }^{1}$ Faculty of Medicine and Surgery, University of "Tor Vergata" Rome, Italy

${ }^{2}$ Faculty of Sport Science, University of Verona, Italy

${ }^{3}$ CONI - Italian Regional Olympic Committee, Sardinia, Cagliari,

Italy

${ }^{4}$ Faculty of Informatics Engineering, University of "La Sapienza", Rome, Italy

Leonardo Da Vinci in the 15th centuries was the first to study the human locomotion. Since that, walking and running gait has aroused strong interest in both sport and rehabilitation science but they usually have used expensive devices. The present study compare two devices: an highspeed camera (Casio FH20 - $210 \mathrm{~Hz}$ ) and new low cost Infrared-Led (IL) device MarkWiiRTM (Padulo J and Vando S, Latina, Italy) to assess the walking and running gait. On a treadmill, ten males performed different walking speeds $(1<6 \mathrm{~km} \bullet \mathrm{h}-1)$ and running speeds $(10<13 \mathrm{~km} \bullet \mathrm{h}-1)$ for 60 " with $1: 1$ work to relief ratio. During the test, a marker attached to the malleolus was recorded simultaneously with the two devices. The displacement of the marker on the two axis, $\mathrm{X}$ and $\mathrm{Y}$, was extracted using two different software's KinoveaTM 0.8.15 and CoreMeterTM for the Camera and IL respectively. After aligning the two signals we interpolated the camera signal raising it from $210 \mathrm{~Hz}$ to $100 \mathrm{~Hz}$ and we normalized the signals and calculated the difference between both devices. Bland-Altman plots of the walking speeds showed an average error of $0.24 \pm 0.52 \%$ and $1.5 \pm 0.91 \%$ for the $\mathrm{X}$ and $\mathrm{Y}$ axis respectively. While the difference of running speeds showed a $0.67 \pm 0.33 \%$ and $1.26 \pm 0.33 \%$ for the $\mathrm{X}$ and $\mathrm{Y}$ axis respectively. The calculation of the $\mathrm{Z}$ value is less than 1.96 in all conditions, demonstrating that the two measures are similar both in the $\mathrm{X}$ axis and in the $\mathrm{Y}$ axis. These findings suggest that the MarkWiiRTM is a valid tool to assess the walking and running gait given that it is portable, cheaper and widely available on the market. Therefore it could become widely used in the sport area and in a clinical setting.
Comparison of two heart rate monitors during an outdoor training session

\section{Scotton ${ }^{1}$, L. Pizzigalli ${ }^{1,2}$}

${ }^{1}$ Scuola Universitaria Interfacoltà in Scienze Motorie, SUISM,

Università degli Studi di Torino, Italy

${ }^{2}$ Centro Ricerche Scienze Motorie, SUISM, Università degli Studi di Torino, Italy

A non professional subject (52 y.o., weight $67 \mathrm{~kg}$, height $170 \mathrm{~cm}$, BMI 23.2), did two training sessions on a free-ride mountain bike Scott Nitrus 20 wearing the heart rate monitor Polar S 725 Tour Edition and the Global Position System (GPS) Garmin Forerunner 305. The aim of the study was to gain more understanding on these instruments (leader of athletic performance analysis) and to evaluate the agreement between their collected data. Findings show that both in a dirt and in an asphalt road the maximal and the median cardiac frequencies (bpm) are the two variables less influenced by the kind of instrument used for the measurement. Analyzing the percentage of means difference among variables acquired by Polar and Garmin system during dirt road trials, only for distance $(\mathrm{km})$ and for mean velocity $(\mathrm{km} / \mathrm{h})$ some differences were reported between the two instruments: $5.8 \%$ and $6.8 \%$ respectively. Only in this condition both these two variables were underestimated with the Garmin system. On the other hand, during asphalt road training sessions the percentage of means difference were: $1.2 \%$ for time (s) and $2.9 \%$ for distance $(\mathrm{km})$. Finally, the distance $(\mathrm{km})$ was found to be the variable measured with more precision by both instruments with a standard deviation in the range $0.01-0.04 \mathrm{~km}$. The study confirms that the use of these instruments needs and objective assessment of measure repeatability, in order to allow the comparison of data collected from different athletes during their sporting practice.

Postural Experience: photographic image in the observation of posture in young hockey players

\section{A. Nart ${ }^{\circ}$, S. Scarpa ${ }^{*}$, B. Vincenzo ${ }^{\circ}$}

'Faculty of Health and Physical Exercise, "Carlo Bo”, University, Urbino, Italy

* Department FISPPA (Philosophy, Sociology, Education and Applied Psychology) University of Padua, Italy

Introduction: In the context of sports activities, postural observation is of considerable importance for the early detection of possible morphological alterations related to mental and physical development in adolescents as well as to analyze any morpho-functional alterations distinctive of asymmetric sports. Recent studies show that back pain in young athletes is often associated with the type of sport they play and its duration. This study aims to examine how the practice of roller hockey, which in literature is considered an asymmetrical sport, may affect young athletes' posture.

Method: Study participants were 80 boys aged between 10 and 14 years, divided into two groups, namely: a control group (CG), composed of students of some secondary schools, and a group of young athletes which had been practising roller hockey for at least 2 years (HG). The postural analysis was performed with the Postural Experience method, a protocol of contextual evaluation of dysmetrias by means of angular measurements of the platform balances on the three planes of space, with the use of software - Podia XP - following the instructions provided by IFSPS (International Federation on Stabilometry and Posturology Society). 
The procedure included a guided protocol for the definition of reference markers, identifiable in the photos taken. For each boy were 4 pictures taken corresponding to postures on the frontal and sagittal planes.

Results: The results of the analyzes concerning the full sample showed for each variable values reater than zero, i.e. values that do not correspond to a "correct posture". The highest values occurred in the variable that takes into account the position of the head on the sagittal plane, the angle included between the vertical line passing through the lateral malleolus and the axis passing through the malleolus and the internal auditory meatus (SP: acoustic meatus) with a mean equal to 3.08 degrees, which can mean head anteposition. Observing the averages relating to the two groups, on the contrary, the highest values were found for the hockey group (HG).

The analysis of variance showed significant differences only for the variable corresponding to the deviation of the shoulder on the sagittal plane, the angle included between the vertical line passing through the lateral malleolus and the axis passing through the malleolus and the middle point of the shoulder (SP: shoulder) with p-value equal to .016 .

Conclusion: The results of this study highlighted that the playing roller hockey could adversely affect the young athlete's posture and in particular the anteposition of the shoulders. With regard to the asymmetry induced by this discipline, on the other hand, no postural alterations attributable to the latter were observed.

References

- Jackson C, McLaughlin K, Teti B, (2010) J Pediatr Health Care, 25: 284-93

- Santos M, Silva M, Sanada L, Alves C (2009) Rev Bras Fisioter, 13: $350-5$

\section{Active videogames: valid alternative to sedentary lifestyle?}

\author{
F. Rastelli ${ }^{1,2}$, A. Di Fabio ${ }^{1}$, M. G. Vinciguerra ${ }^{1}$, S. Chiavaroli ${ }^{1,3}$
}

${ }^{1}$ Dip. Scienze Chimiche Applicate e Biotecnologiche, Facoltà di Scienze Motorie, Università degli Studi di L'Aquila (AQ), Italy ${ }^{2}$ Istituto di Bioimmagini e Fisiologia Molecolare del Consiglio Nazionale delle Ricerche, Segrate (MI), Italy

${ }^{3}$ IRCCS Neuromed, Pozzilli (IS), Italy

Introduction: Physical inactivity and excess caloric intake are associated with obesity; they are well-established risk factors for many chronic conditions and are estimated they cause 1.9 million premature deaths globally per year.

There have been many efforts in the school, workplace, and home to increase daily physical activity. One of the latest approaches consists in using active videogames (AV): their feature is the movement of the player such as would occur in "real-life" exercise participation.

Aim of this study was to verify the efficacy of AV as a safe and reliable method to reduce physical inactivity.

Methods: 12 untrained students, 6 males $(24 \pm 4 \mathrm{yr}, 74 \pm 24 \mathrm{~kg}, 175 \pm 10$ $\mathrm{cm})$ and 6 female $(26 \pm 8 \mathrm{yr}, 63 \pm 7 \mathrm{~kg}, 166 \pm 5 \mathrm{~cm})$, performed 3 test sessions in different days. During the first session, a $15^{\prime}$ incremental submaximal test on a treadmill was performed to estimate maximal oxygen uptake (VO2max). During the other two sessions, each subject performed, in random order, 10' free-running outdoors (FR) or 10' of AV ("Jojjing plus", Nintendo Wii Fit ${ }^{\mathrm{TM}}$ ). Both exercises were performed at intensities freely chosen by the subject; the only indication for the subject was to perform the exercise without stopping. For FR and AV the following parameters were considered: average energy expenditure of 10' exercise as gross (EE) and net (netEE) value, peak values of oxygen uptake (VO2peak) and heart rate (HRpeak) and rate of perceived exertion (RPE), indicated by the subject at the end of exercise.

Comparison between two exercise types was made using Wilcoxon test, while the Mann-Whitney U test was adopted to evaluate gender differences. Results: In gender comparison there were no significant difference, as well as in the comparison between FR and AV for baseline values. EE, for both sexes, was higher $(\mathrm{p}<0.05)$ in FR (males: $13.6 \mathrm{Kcal} / \mathrm{min}$; females: $11.0 \mathrm{Kcal} / \mathrm{min}$ ) compared to AV (males: $9.7 \mathrm{Kcal} / \mathrm{min}$; females: $8.0 \mathrm{Kcal} / \mathrm{min}$ ); the same significant differences was recorded for netEE (FR: $12.0 \mathrm{Kcal} / \mathrm{min}$ for men and $9.8 \mathrm{Kcal} / \mathrm{min}$ for women; AV: 8.1 $\mathrm{Kcal} / \mathrm{min}$ for men and $6.7 \mathrm{Kcal} / \mathrm{min}$ for women). During FR, VO2peak and HRpeak reached values close to their maximum references: respectively for men and women, $87.4 \%$ and $90.9 \%$ of VO2max, and $90.8 \%$ and $93.3 \%$ of HRmax; these values was significantly higher of those recorded during $\mathrm{AV}$ : respectively for men and women, $69.9 \%$ and $74.4 \%$ of VO2max, and $77.3 \%$ and $88.2 \%$ of HRmax. Finally, also RPE values were found to be higher $(\mathrm{p}<0.05)$ in FR (FR: 3.5 both for men and women; AV: 2.8 for men and 2.4 for women).

Conclusions: FR leads to higher EE that AV; AV, however, has EE comparable to that of moderate activity, without reaching high peaks of intensity such as FR. Therefore AV seems more suitable as a safe and reliable method to promote physical activity among untrained individuals.

References

1. Rütten A. Soz Praventivmed. 2004; 49(4): 281-289.

2. Lanningham-Foster L. J Pediatr. 2009; 154(6): 819-23.

3. Beers EA. Eur J Appl Physiol. 2008; 103: 353-60.

\section{Session 9. Testing and Performance evaluation - Adapted Physical Activity - Sport Nutrition}

\section{Predictive monitorscreen of overtraining by analysis of salivary Cortisol, IGA and testosterone and their association with genetic polymorphisms in gymnastics}

\section{G. Innocenti, G. Morucci, J. J.V. Branca, S. Pacini, M. Gulisano}

Dipartimento di Anatomia, Istologia e Medicina Legale, Università di Firenze, Florence, Italy.

It is well known that an excessive physical activity may stress the organism determining unbalanced hormonal orders that can negatively affect the state of optimal health and therefore the sporting output. In particular during physical exercise the serum Cortisol and Testosterone concentrations increase with the intensity of the exercise while IgA levels decrease.

On this basis, there is the possibility the determine if the program of preset physical exercise evokes an abnormal reaction of adaptation of the organism.

Since physical exercise and athletic performance are strongly related to individual genetic traits, we think that the possibility of customizing the athletic training on the basis both of genetic and of biomarkers involved in physical stress may be a tangible fact.

In this study we evaluated the role of several sport-related gene polymorphisms in affecting the physical stress (monitored by salivary biomarkers) during a standard training session. Furthermore we evaluated the feasibility of salivary tests during training session both in terms of athlete's discomfort both in terms of laboratory quality sample.

Eighteen male gymnasts (age $18.2 \pm 5.4$ years) were genotyped for the following

sport- related gene polymorphisms: Angiotensin Converting Enzyme (ACE - I/D), i j-actinin 3 (ACTN3 - RR/RX) and Vitamin D Receptor (VDR - BsmI, FokI, TaqI, ApaI).

Salivary levels of Cortisol, IgA and free Testosterone were measured before and after a standard training session. Saliva samples were collected 
by Saliva Collection Device (Grifols, Italy) and measure of salivary markers were performed by a specific measure kit (Grifols, Italy). Data were analyzed by Pearson analysis, Student $t$ test, and variance analysis (ANOVA). Results were significant when $\mathrm{p}<0.05$.

Our data show that in the majority of the examined athletes levels of salivary Testosterone increase after a training session and a strong correlation $(\mathrm{p}<0.05)$ between salivary levels of this marker before and after the training session exists. On the contrary no correlation exists between Cortisol and IgA levels before and after the training session and no significant correlation was found between genetic polymorphism and levels of salivary markers.

These preliminary results have to be interpreted considering the small size of the examined athletes group $(n=18)$. Since we are aware of the fact that in this kind of study the size of the examined population strictly affects the results, we are now extending this study to a larger group of athletes.

In fact we have reasonable scientific basis to postulate a strong link between genetic, salivary biomarkers and overtraining and we are working to get our main target: giving to athletic trainers strong tools to plan a customized training sessions avoiding a stress- related overtraining and, at the same time, adjusting the training on the basis of individual genetic profile.

Keywords. Cortisol, IgA, Testosterone, genetic polymorhisms, physical training.

\section{Monitoring of the training load in a professional football team M. Beato ${ }^{1}$, G. Brignardello ${ }^{2}$, F. De Vita ${ }^{2}$, L. Bortolan ${ }^{1}$, L. Posenato ${ }^{2}$, C. Tarperi ${ }^{l}$, F. Schena ${ }^{l}$}

${ }^{1}$ Department of Neuroscience and Kinesiology, University of Verona, Verona, Italy

${ }^{2}$ Technical \& Medical Staff, AC ChievoVerona, Verona, Italy

Aim: The aim of this study was to analyze the intensity of training sessions during the regular season of a professional football team. The monitoring of workload during training and competition represent a relevant control factor for high level football player and it could be evaluated by the system GPS1.

These sprint activities can be also monitoring using accelerometer. Although this solution is not able to esteem the information on the ability of soccer players to perform specific bouts of during exercise 2 , it can measure limited movements and jumps not recognized by the GPS system.

Method: 20 professional football players of the Chievo Verona FC team were monitored for the season 2011/12 from July to April. The goalkeepers were excluded from the study. The following parameters were evaluated: - training load index (TL), obtained by processing data collected by individual triaxial accelerometer wore by players during weekly session; - training volume index (TV), derived from the individual mean time ( $\mathrm{min}$ ) performed by the team, excluding the total time used for warm-up.Because it was not possible to detect all training sessions we analyzed data as an average per each measured trial and reported as mean $\pm \mathrm{SD}$. The statistical analysis were performed by using one-way Anova and post hoc multiple comparison versus first month of training considered as control (adjusted Bonferroni t-test). The confidence interval chosen was $95 \%(\mathrm{p}<0.05)$.

Results: We have found by the statistical analysis a significant difference between TL in July (chosen as control situation) versus November $(+83.4 \%)$ and February $(+88.8 \%)$ while there was no statistical difference between July and other months. TV show not significant changes along the whole agonistic season even if the percent changes of work were in a range of $25 \%$ with the lower in peak in January in close relationship with the number of matches played.

Conclusion: After analysis we can say that the average volume of train- ing sessions devoted to improve the overall aerobic capacity of the players does not significantly change during the season. The average training load, significantly related to the ability of repeated sprints, improves in the months of November and February. The accelerometric method has proved a useful control tool for work scheduling in professional football.

\section{References}

1. Randers MB et al. (2010) Application of four different football match analysis systems: A comparative study Journal of Sports Sciences, January 15 th; 28(2): 171-182

2. Carling C. et al (2012) Analysis of repeated high-intensity running performance in professional Soccer. Journal of Sports Sciences, February 30(4): 325-336

\section{Reliability of direct and indirect measurements of one repetition maximum}

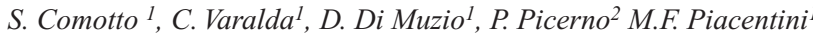 \\ ${ }^{1}$ Department of Human Movement and Sport Sciences, University \\ of Rome "Foro Italico", Rome, Italy \\ ${ }^{2}$ SENSORIZE S.r.l., Rome, Italy
}

Aim: Direct measurement (DM) of one repetition maximum (1RM) has been studied and is a reliable method to measure maximal strength. $1 \mathrm{RM}$ can also be predicted indirectly by performing a submaximal test. The aim of the present study was to evaluate the validity and the reliability of the prediction of 1RM assessed with DM and indirect measurements.

Methods: 18 subjects (14M;4F, $23 \pm 2 \mathrm{yrs}$ ), with no experience in weight lifting, were recruited for the present study. The participants performed 4 trials (T1, T2, T3 and T4) for the upper limbs (UL) at the chest press and lower limbs (LL) at the leg press. Each trial consisted in a DM and an indirect measurement for UL and LL performed in a randomized order. The DM was performed with an incremental test arriving at the load that participants could lift only once. The prediction of 1RM was assessed using the 6RM and a conversion table (CT) [1], the Brzycki formula (BF) [2] and the FreePower (FP) (Sensorize S.r.l., Italy). FP is a software that inputs acceleration data measured by a wireless inertial measurement unit fixed to the weight stack of the machine through a magnetic pocket. The software estimates $1 \mathrm{RM}$ by using a mathematical relationship between force, power and velocity measured in correspondence of at least three increasing loads [3]. Repeated measures ANOVA $(\mathrm{P}<0.05)$ was used to evaluate differences between trials and between $\mathrm{BF}$, $\mathrm{CT}, \mathrm{FP}$ and DM. Intraclass correlation coefficients (ICC) were used to evaluate reliability. Retest correlation was measured by Pearson correlation coefficient $(\mathrm{P}<0.05)$.

Results: For UL, differences emerged for 1RM between T1, and T2, T3 and T4 $(\mathrm{P}<0.05)$ and between T4 and T2 and T3, in BF, CT, FP and DM $(\mathrm{P}<0.05)$. Thus, to evaluate the ICC only T3 and T4 were considered for each measurement. A high ICC (range 0.979-0.997) was found for UL. In addition, the retest correlation was $>0.969$. Moreover, differences in each trial emerged between $\mathrm{BF}$, and $\mathrm{CT}$, FP and $\mathrm{DM}(\mathrm{P}<0.05)$. No differences emerged between FP and DM. Similar results are evident for LL. In particular, differences emerged for 1RM between T1, and T2, T3 and T4 $(\mathrm{P}<0.05)$ in BF, CT, FP and DM. To evaluate the ICC, T1 was excluded. A high ICC (range 0.990-0.998) was found for UL. In addition, the retest correlation was $>0.958$. Finally, for $L L$ differences in each trial emerged between $\mathrm{BF}$ and $\mathrm{CT}, \mathrm{FP}$ and $\mathrm{DM}(\mathrm{P}<0.05)$. Also for $\mathrm{LL}$ no differences emerged between FP and DM.

Conclusions: DM of 1RM is time consuming, and may lead to muscle soreness as well as temporary deterioration of the function of the tested muscles. Differences found for T1 and T2 demonstrate that a familiarization session is needed both for DM and indirect measurements. 
Differences emerged between $\mathrm{CT}$ and $\mathrm{BF}$, and the lack of differences between DM and FP demonstrated that FP is the best reliable and useful method to determine 1RM.

\section{References}

1. Baechle et al. (2000) Human Kinetics

2. Brzycki (1993) Journal of Physical Education, Ricreation and Dance 64:88-90

3. Squadrone et al. (2012) JSMPF 52:40-6

\section{A time-space distribution analysis of action in foil athletes: a preliminary study on 2011 World Championship}

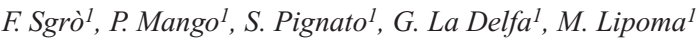 \\ ${ }^{I}$ Engineering, Architecture and Physical Activities Faculty, University of Enna, Enna, Italy}

Introduction:The scientific research in fence sport has been conducted in several fields, such as biomechanical, physiological and medical (Gholipour et al., 2008; Tsolakis\&Vagenas, 2010; Roi\&Bianchedi,2008; La Delfa et al., 2012) but, in the best of our knowledge, no one was conducted in the specific field of performance analysis. In this work we will address if there are some tactical-based elements related with springboard position or each one of the three-game-period in the performance of foil elite athletes.

Methods: The foil is one of the three types of weapon include in fencing and it is defined as a "formal weapon", because the winning stroke must be conducted by means specific rules and in chronological frame. In order to analyse the foil matches, following the hint of the Coaches of Italian Foil Olympics Team, we will define a specific notational analysis platform, based on a software for video-analysis and KorePAS, an ad-hoc software for computerised notational analysis (Sgrò et al., 2010). We analysed 14 elite athletes involved in the latest world championship (8 males and 6 females), which played 8 matches and performed 595 actions totally. For each action we noted: the result of bout, the results of action, the type of action, the body target, the position on springboard and the period. The action result was classified in: winning, loosing and invalid. The springboard was divided in three parts, called: defensive, centre and attack position; furthermore, for the analysis, the match was divided in 3 periods of 3 minutes each one.

Results: According to Nevil et al. (2002), we used non-parametric statistics tests. Initially we organize the data in two contingency tables and we applied a chi-square test and a correlation analysis, in order to evaluate the characteristics of the data distribution. In each table data were grouped by the result of bout, in row there were the results of action while in the column the springboard position and the period of game respectively. A successive chi-square test was applied in a third contingency table where the position data was divided in two groups: centre and left-right side. In all chi-square tests we obtain a non-significance level of results and for each cell crossing we didn't obtain interesting standardize residual values.

Discussion: The notational analysis, unify with appropriate statistical methods, can be used in fence as support both for the tactical decision that to establish a specific training protocol. The result obtained with chisquare test show that the distribution of strokes both in space that in time analysis has a casual trend, so specific training isn't suggest to improve the performance of the athletes linking with these two variables. Furthermore, for the space analysis, no relevant notation for the performance improving was found also dividing the springboard in only two side.

Acknowledgements: Italian Federation of Fencing for the support to this research.

\section{CoM displacement influences the energetic cost of locomotion during the double poling technique}

\section{Zoppirolli, B. Pellegrini, A. Savoldelli, L. Bortolan, F. Schena.}

Neurological, Neuropsychological, Morphological and Movement Science Department, (University of Verona - CeRiSM (Research Center of Sport Mountain \& Health, Rovereto), Italy

Introduction: Double poling technique (DP) is a particular type of skiing locomotion due to the extensive use of the upper body. Cost of locomotion is generally related to metabolic adaptations to training, but also biomechanical strategies can concur to set the amount of energy spent to travel at a given speed. The aim of this investigation was to analyze metabolic and biomechanical characteristics of two differently skilled groups of cross-country skiers, in relation to the energetic cost of DP locomotion.

Method: Eleven recreational (R) and ten high level (HL) cross country skiers were ask to ski on a treadmill with the DP, performing 3 steady state trials $(10,12$ and $14 \mathrm{~km} \bullet \mathrm{h}-1)$. For each trial energetic cost of locomotion (EC) was calculated by considering the contribution of aerobic and anaerobic metabolism. Individual EC was computed as the mean values of the trials. For the fastest speed we computed the 3-D coordinates of the body centre of mass (CoM), during 30 seconds of acquisition. The moment acting on a skier at each instant of time on the sagittal plane was calculated using the formula: $\mathrm{M}=\mathrm{r}^{*} \mathrm{~F}^{*} \sin ($ ), where $\mathrm{r}$ is the line joining CoM and the medial point between feet CoM, F is body weight and is the angle of $r$ with respect to the vertical. The force applied to the ground through the poles was recorded as well, and the average poling force exerted during a mean cycle (PFavg) was calculated.

Results: EC was significantly higher for the RG than for HLG $(4.51 \pm 0.03$ and $3.73 \pm 0.24 \mathrm{~J} * \mathrm{~m}-1 * \mathrm{~kg}-1$ respectively; $\mathrm{p}<0.01)$. Percentage anaerobic contribution was significantly higher for RG than for HLG in all the trials $(p<0.01)$. From biomechanical point of view we noticed that at each cycle $\mathrm{M}$ reaches its maximum just before the starting of the poling phase. Between the groups significant differences were observed for the maximal values of $\mathrm{M}(78.6 \pm 20.0 \mathrm{Nm}$ for $\mathrm{RG}$ and $98.6 \pm 22.5 \mathrm{Nm}$ for HLG; $\mathrm{p}<0.05)$ and for PFavg $(70.2 \pm 7.5 \mathrm{~N}$ for RG and $76.8 \pm 7.0 \mathrm{~N}$ for HLG; $p<0.05$ ). Within each group, inverse significant relationships were found between EC and maximal $\mathrm{M}$ values $(\mathrm{r}=-0.80$ and $\mathrm{p}<0.01$ for $\mathrm{RG} ; \mathrm{r}=-0.66$ and $\mathrm{p}<0.05$ for $\mathrm{HLG}$ ).

Conclusion: Less skilled skiers need more metabolic power to sustain a given mechanical load. Furthermore they use a larger percentage of energy deriving from the anaerobic metabolism. The groups are characterized by biomechanical differences as well: high level skiers presented higher moment values before pole plant. This biomechanical characteristic probably indicated that a more advanced position of CoM probably allow the skiers to load the poles during the body weight fall; this mechanism would economize the propulsion resulting in a lower cost of skiing. The significant relationship found within the groups between the EC cost and the maximal moment values, further support these speculation. This locomotion strategy should help to save upper limb muscle work during the poling phase, contributing in the limitation of blood lactate production. 


\section{A contribution to the Italian validation of the Physical Activity Questionnaire for Older Children (PAQ-C)}

\section{E. Gobbi, I. Ferri, A. Carraro}

\section{FISPPA Department, University of Padua, Padua, Italy}

Background: Knowledge of children' physical activity levels is fundamental in interdisciplinary health care and educational interventions. In recent literature it is underlined the need to adopt objective measure of physical activity, however the use of self-report instruments are still the most used in large population studies because of their cost-effectiveness.

The Physical Activity Questionnaire for Older Children (PAQ-C, Crocker et al., 1997) is a questionnaire designed to assess habitual moderate to vigorous physical activity levels of school aged children from grades four to eight. It is a 7-day recall instrument, consisted of 10 items and it lastly scores by means of 9 items between 1 (low physical activity) and 5 (high physical activity). In order to contribute to the validation of the PAQ-C in the Italian population, an Italian version of the PAQ-C was obtained by means of a back translation procedure.

Materials and method: One thousand one hundred and forty-six children of 4th and 5th grade completed the Italian version of the PAQ-C during a physical education lesson, with a class administration method, in April. As indicated in the scoring manual, pupils who indicated that they cannot perform the normal physical activity during the previous week were excluded from the analysis. The considered group consisted of 576 boys and 517 girls with a mean age of 9.3 years $(\mathrm{SD}=0.6$ years). General test score characteristics of the Italian version of the PAQ-C were investigated. Descriptive analysis of items were examined separately for boys and girls as well as for the total group.

Results: MANOVA reported a significant gender effect (Wilks Lambda $=0.957 ; \mathrm{p}<.001)$. Univariate ANOVA shown significant differences by gender on all items scores, with boys scoring higher than girls $(\mathrm{p}<.01$ and $p<0.05$ on item 4 ), with exception for the first item in which boys and girls reported similar values. An independent sample t-test on the total PAQ-C score, reported values significantly higher for boys $(\mathrm{t}(1091)=$ $6.04, p<0.01)$. Scale reliabilities for girls $(\alpha=0.76)$, boys $(\alpha=0.71)$ and total group ( $\alpha=0.74$ ) were acceptable. Mean corrected item-total scale correlations were .45 when considering the whole group, 0.42 for boys and 0.47 for girls.

Discussion: The Italian version of the PAQ-C resulted homogeneous with the original version. Results of the descriptive analysis reported a low mean value and variance for the first item and a high mean value for the physical education item, as in the original version of Crocker and Colleagues (1997).

The Italian version of the PAQ-C appears a useful, inexpensive instrument to assess habitual moderate to vigorous physical activity in children large population studies. However, further research is necessary to confirm the reliability of the questionnaire in test-retest administrations, over long-term periods and in comparison with objective data (e.g. accelerometers and pedometers).

Performance enhancing substances and supplements consumptions among Italian gym users: prevalence and effects of a knowledge-based intervention

C. Pesce ${ }^{1}$, L. Leone $e^{2}$

${ }^{1}$ Department of Human Motor Science and Sport, University of Rome Foro Italico, Italy

${ }^{2}$ Department of Human Motor Science and Sport, University of Rome Foro Italico, Italy
Background: Strong correlations between ergogenic substances, fitness legal supplements and anabolic androgenic steroid use among athletes and non athlete young sportives recently emerged (Backhouse et al., 2011). The so called 'gateway theory' (Hildebrandt $T$ et al. 2012) gives an explanation of this association considering ergogenic substances as precursor of doping. Also among Italian adolescents an association between protein and vitamin supplements, no prescription drugs (Paoli, Donati 2012, Pesce et al., 2004 ; Pesce \& Donati, 2009 , Pesce, 2010 ) and doping substances has been reported (Lucidi et al., 2004). The ISS Istituto Superiore di Sanità and the Anti doping national Commission have commissioned a study to evaluate the effects o an anti-doping campaign aimed at improving correct knowledge among students and gym users (Leone 2012, Leone, Pesce 2012).

Aim: The aims of our study were: (a) identifying data about prevalence of licit and illicit performance enhancing substances (PES), attitudes towards doping, believes and knowledge related to healthy nutrition and use of supplements; (b) evaluating the effects of a knowledge-based intervention about sport and nutrition, doping and consumption of PES. Methods: The survey has been carried out in the period February-April 2012 on a sample of 422 gym users in four Emilia Romagna and Lazio cities. An experimental design, with pre-post interviews and a control group, has been adopted to assess the effects of the intervention.

Results: Vitamin and mineral salts supplements are widely diffused in a sample of gym users aged 15-30years (50\%), ergogenic supplements (creatine, amino acids) is quite high (12\%) and gender related. Pattern of consumptions related to gender, type of sport activity, age, socio-economic class emerged as well annual expenditure for each class of product and place of purchase (Gyms, internet shops, pharmacies). A textbook about nutrition for sportsperson and a DVD about doping were given to a group chosen with random criteria. A theory driven approach of evaluation was used. The paper presents the results of the intervention evaluation and discuss them in the light of a comprehensive approach of health promotion.

Effects of supraphysiological doses of single or combined doping agents in human peripheral blood lymphocytes

\section{E. Imperlini ${ }^{1,2}$, S. Spaziani ${ }^{3}$, A. Mancini ${ }^{1,3}$, A. Alfieri $^{1,3}$, P. Buono ${ }^{1,2,3}$, S. Orrù ${ }^{1,2,3}$}

${ }^{I}$ CEINGE Biotecnologie Avanzate, scarl, Naples, Italy

${ }^{2}$ SDN-IRCCS Foundation, Naples, Italy

3 Dept. DiSIST, University "Parthenope", Naples, Italy

It is widely believed that insulin-like growth factor-1 (IGF-1) is abused by athletes and sport amateurs, either alone or in combination with anabolic androgenic steroids (AASs) such as dihydrotestosterone (DHT). IGF-1 is the main effector for the action of the growth hormone; it has effects on carbohydrate, lipid and protein metabolism in different cell types. DHT, that shows greater affinity for androgen receptor (AR) to respect to testosterone, is responsible for the androgenic effect. Until now, several preclinical studies have demonstrated a direct correlation between tumorigenesis and IGF-1 or DHT abuse (Socas L et al. Br J Sports Med 2005, 39(5):e27; Pollak M. Nat Rev Cancer 2008, 8 (12): 915928.). We have recently demonstrated that supraphysiological doses of DHT activate the drug detoxification process, stimulate cell motility and exert a pro-survival effect in human peripheral blood lymphocytes (Imperlini E et al Proteomics 2010, 10 (17): 3165-75). To gain insight into adverse effects of abuse of single or combined doping agents, we analyzed protein expression profiles in human lymphocytes treated with supraphysiological doses of IGF-1 alone or in combination with DHT using 2D DIGE technology. The differential proteomic analysis showed that 29 and 28 protein spots are differently expressed after IGF-I or IGFI/DHT treatment, respectively (fold change $\geq 1.20$ or $\leq-1.20$; t-test: 
$\mathrm{p} \leq 0.05$ ). The characterization of the differentially expressed proteins will be performed by using mass spectrometry analysis coupled to bioinformatic tools. This study will allow to identify IGF-I/DHT sensitive markers and to define cellular processes affected by the single or the combined treatment.

Psychological well-being and social participation assessment in visually impaired subjects playing Torball: a controlled study

E. Iuliano ${ }^{1}$, G. Aquino ${ }^{1}$, A. Giombini ${ }^{1}$, C. Battaglia ${ }^{2}$, G. Fiorilli ${ }^{1}$, A. Di Cagno ${ }^{1}$, G. Calcagno ${ }^{1}$

${ }^{I}$ Department of Medicine and Health Sciences, University of Molise, Italy

${ }^{2}$ Department of Health Science Italian University of Sport and Movment Rome, Italy

Introduction: Visually impaired subjects tend to have lower level of social participation and psychological well-being. Physical activities and sport could be a way to bridge the gap between subjects with and without disabilities and to learn social integration increasing well-being perception.

Aims: The aim of this study was to evaluate the differences between torball players and non-players blind subjects, in social participation and psychological well-being.

Methods: Thirty-one blind male subjects were recruited, 18 Torball play-

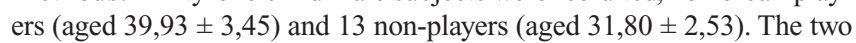
groups were homogeneous for the visual disability and school level instruction. The participants were evaluated in three different areas: (a) social participation level, (b) psychological well-being and (c) psychological disorders. Each of this areas were examined respectively using three validated self-report questionnaires: Participation Scale, Psychological WellBeing Scale [PWB Scale] and Symptom Checklist 90 R [SCL-90-R].

Results: The statistical analysis showed significant differences in overall the three areas between the two groups. The social restriction score of the Participation Scale in the non-players group was significantly higher $(p<.001)$ than the players group. The PWB Scale showed significant differences $(\mathrm{p}<.001)$ in 5 dimensions (Positive Relations with Others, Environmental Mastery, Personal Growth, Purpose In Life, SelfAcceptance), with better scores for the sportive group; no significant differences in the Autonomy dimension were found. In the SCL 90, the scores of the sportive group were significantly lower in 8 symptomatic dimensions (Obsessive-compulsive disorder, Interpersonal sensitivity, Depression, Anxiety, Phobic anxiety, Paranoid, Psychoticism, Sleep disorders); no differences in the Somatization and Hostility dimensions were found. Similarly the three global scores of the SCL 90 (Global Score Index, Positive Symptom Total, Positive Symptom Distress Index) were significantly better for the sportive group than controls $(\mathrm{p}<.001)$.

Discussions: The main results of this study indicated that torball athletes with visual impairment had a higher level of social participation and psychological well-being than controls. Torball, as a team sport, created occasions of social interaction and friendly relationships for blind subjects.

\section{References}

1. Morelli T., Folmer E., Foley J.T., Lieberman L. (2011) Improving the lives of youth with visual impairments through exergames; Research and practice in visual impairment and blindness: 4(4): 160-170

2. Movahedia A., Mojtahedia H., Farazyanib F. (2011) Differences in socialization between visually impaired student-athletes and non-athletes; Research in Developmental Disabilities: 32(1): 58-62
3. Valliant P. M. (1985) Psychological Impact Of Sport On Disabled Athletes; Psychological reports: 56: 926-929

\section{Soccer practice as vehicle to improve sporting performance and psychophysical health in psychotic subjects}

G. Battaglia ${ }^{1,2}$, M. Bellafiore
G. $^{1,2}$, M. Inguglia $^{3}$, M. $^{2}$ Alesi $^{4}$,

${ }^{1}$ Department of Sports Science (DISMOT), University of Palermo, Italy

${ }^{2}$ Regional Sports School of CONI Sicilia, Italy

${ }^{3}$ Local Health of Palermo, Italy

${ }^{4}$ Department of Psychology, University of Palermo, Italy

In recent years there has been a growing attention in the use of soccer as a vehicle to improve mental health. Local health organizations are beginning to understand that soccer may be an effective way to promote good mental health and help increase access to, and uptake of, services for mental health service users (Pringle 2009). The aim of this study was to investigate effects of soccer practice on the psychophysical perception and performance in psychotic subjects. Eighteen overweight male subjects, 10 trained psychotics [TPs] (age: $36+/-6$ years; height: $164+/-7.00 \mathrm{~cm}$; weight: $77.44+/-13.60 \mathrm{~kg}$; BMI: $28.55+/-3.70$ ) and 8 no-trained psychotics [NTPs] (age: $35+/-5$ years; height: $163+/-$ $4.00 \mathrm{~cm}$; weight: $76.71+/-9.75 \mathrm{~kg}$; BMI: $28.65+/-2.62$ ) of Local Health of Palermo (Sicily, Italy) participated in this study. TPs were trained for 8 weeks by two sessions/week. In particular, every trained session included: a recording phase $(\sim 10 \mathrm{~min})$, a social interaction phase $(\sim 10 \mathrm{~min})$, a warm up period $(\sim 20 \mathrm{~min})$, a training period $(\sim 50$ min) including soccer technical-tactical exercises and a football game, cool down period $(\sim 10 \mathrm{~min})$ and a feedback phase $(\sim 10 \mathrm{~min})$. NTPs did not perform any sport activity during the experimentation. Physical condition and performance of the subjects have been evaluated by body weight (BW), body mass index (BMI), 30 meter sprint test $(30 \mathrm{mST})$ and slalom test running with ball (STB) between 5 cones located $50 \mathrm{~cm}$ apart. Instead, psychophysical perception from psychotics was studied using the SF-12 health status questionnaire measuring self-rated health (Faulkner $\mathrm{G}$ et al., 2007). It investigated the summary measures PCS-12 (physical domain of SF-12, scores ranging from 0 to 70 ) and MCS-12 (mental domain of SF-12, scores ranging from 0 to 70). All data were acquired before and after the experimentation. We found that TPs improved PCS-12 and MCS-12 scores and $30 \mathrm{mST}$ and STB performances than NTPs group after the training period. Moreover, TPs group decreased BW and BMI than NTPs after the experimental period.

Soccer practice appears to be able to improve psychophysical health in psychotic subjects. Indeed, our study showed that programmed physical activity can reduce antipsychotic medication-related weight gain and improve psychophysical perception and performance in psychotic subjects. This because soccer, in agreement with Pringle (2009), could be seen as an organized social phenomenon able to promote a sporting therapeutic and healthy antagonism.

\section{References}

- Faulkner G, Cohn T, Remington G, Irving H (2007) Body mass index, waist circumference and quality of life in individuals with schizophrenia. Schizophr Res 90(1-3):174-8

- Pringle A (2009) The growing role of football as a vehicle for interventions in mental health care. J Psychiatr Ment Health Nurs 16(6):553-7 


\section{Session 10. Functional Adaptation to Exercise - Adapted Physical Activity - Mental Training}

\author{
Physiological demands in mountain biking: a new concept \\ of assessment \\ L. Nadalet ${ }^{l}$, N. Vettorello ${ }^{I}$, Y.O. Schumacher ${ }^{2}$, D. Nonis ${ }^{1}$ \\ ${ }^{1}$ Department of Biomedical Sciences for Health, University of Milan, \\ Italy \\ 2 Department of Sports Medicine, University of Freiburg, Germany
}

Introduction: Mountain biking (MTB) racing is a high demanding discipline where MTB athletes competing at their maximal individual Power Output (PO) throughout the race. The PO is closely correlated to the course profile and frontal position in the group (steep climbs/forward position=higher PO), therefore there are a lot of PO Variations (POV) during races. From basic physics, it become clear that repetitive increases in speed or PO are physiologically much more demanding than a constant $\mathrm{PO}$ over time. It means the cyclists have to increase many times the PO producing high levels of muscle force. To date the mean PO value for a given section (laps) or time is used to evaluate the training load using a common software (Golden Cheeta, Training Peaks), as consequence this does not provide any information about POV and therefore it does not reflect the real MTB effort of the athlete. The purpose of this study is to develop a new method and system to evaluate POV during a race, in order to better understand the importance of such power output variations.

Method: For this purpose, a new software was developed to quantify POV along recorded data through common power meter (SRM, Powertap, Quark, Power+). The software displays the real number (numerosity, $\mathrm{n}^{\circ}$ ), amplitude (watts) and duration (seconds) of POV. By these means, it is possible to obtain the POV in function of race time, in order to quantify the number of times that cyclist increases, decreases and maintains stable the PO. The firsts $10 \mathrm{~min}$ of one athlete during MTB World Cup race (Offenburg 29.05.2011) were analyzed to briefly demonstrate the software utilities.

Results: 206 POV were found, of the duration: $1 \mathrm{sec}\left(\mathrm{n}^{\circ}: 69<400 \mathrm{~W}\right.$ and $73>400 \mathrm{~W}), 1.5 \mathrm{sec}\left(\mathrm{n}^{\circ}: 33<400 \mathrm{~W}\right.$ and $\left.21>400 \mathrm{~W}\right)$ and $2 \mathrm{sec}\left(\mathrm{n}^{\circ}\right.$ : $4<400 \mathrm{~W}$ and $6>400 \mathrm{~W}$ ). To allow a comparison of the data recorded, the PO was $440 \pm 219 \mathrm{~W}$, averaged by Excel (mean and SD).

Discussion. The new software returns several information, such as the high numbers of POV, mainly groups at low time length (1-2 sec). As expected length and amplitude of POV are indirectly related. Despite the mean PO gives only one value, it cannot be neglected in order to evaluate the absolute and relative effort in a different unit of measure. The method presented contribute adding worth at the mean $\mathrm{PO}$ value. It looks inside the mean PO in order to reflect the high demanding discipline, furthermore, it is valuable from a scientific point of view, so as to better describe the performance model of MTB. Concluding, it might help coaches to improve the assessment methods and plan training accordingly. In this way technical staff could enhance the performance. However, further investigations are necessary to provide the validity and reliability of the new software.

\section{High-volume and high-intensity training in masters swimmers}

L. Pugliese ${ }^{1}$, M. Bonato ${ }^{1}$, G. Bellistri ${ }^{2}$, A. La Torre $^{\text {I }}$, M. Marzorati ${ }^{2}$, M. Maggioni ${ }^{1}$, A. Parisi ${ }^{3}$, F. Pigozzi ${ }^{3}$, S. Porcelli ${ }^{1,4}$

${ }^{1}$ Department of Sport, Nutrition, Health Sciences, Faculty of Exercise Sciences, University of Milan, Italy
${ }^{2}$ Institute of Bioimmaging and Molecular Physiology, National Research Council, Italy

${ }^{3}$ Department of Health Sciences, University of Rome "Foro Italico", Italy

${ }^{4}$ Faculty of Exercise Sciences, Telematic University of San Raffaele, Italy

Introduction: Training volume of swimmers averages approximately 20-30 training hours per week depending on the individual performance level (Sharp 2000). Moreover, a great part of the weekly training volume is usually performed at low intensity (Maglischo 2003; Mujika 1998; Sharp 2000). Recently the benefits on performance and physiological responses of high-intensity training has been reported in several athletes (Laursen \& Jenkins 2002). As for high-intensity training in swimming, few studies mainly focused on youngs are available (Faude et al. 2008, Sperlich et al. 2010).

The aim of the present study was to evaluate in high-level masters swimmers the effects of different training programs on performance. Methods: Twelve master swimmers (males, $32 \pm 5 \mathrm{yr}$ ) participated to 15 weeks of training. In the first 6 weeks they performed a high volume-low intensity training (HV-LI), swimming about $14 \mathrm{~km} /$ week. In the last 6 weeks, after 3 weeks of tapering, they performed a low volume-high intensity training (LV-HI) of about $7 \mathrm{~km} /$ week. All swimmers were tested before (PRE), after HV-LI and after LV-HI. In laboratory, they performed an incremental arm exercise test until exhaustion (891E, Monark, Sweden) in order to assess peak cardiovascular and metabolic values. In $25 \mathrm{~m}$ swimming pool, they performed a $2000 \mathrm{~m}$ endurance test and a $7 \times 200 \mathrm{~m}$ protocol for individual anaerobic threshold (Vant) assessment. The best time performance was assessed in $400 \mathrm{~m}$ and $100 \mathrm{~m}$ test.

Results: In relation to PRE: 1) peak oxygen consumption significantly increased both after HV-LI and LV-HI; 2) time performance on $2000 \mathrm{~m}$ was not significantly reduced both after HV-LI and LV-HI; 3) Vant significantly increased only after LV-HI training; 4) time performance on 400m significantly improved both after HV-LI and LV-HI; 5) time performance on $100 \mathrm{~m}$ significantly improved only after LV-HI.

Conclusions: Maximal aerobic power increased after HV-LI and didn't changed after LV-HI. This was associated with a better time performance on $2000 \mathrm{~m}$ and $400 \mathrm{~m}$. Additionally, LV-HI training also improved $100 \mathrm{~m}$ time performance and velocity at anaerobic threshold. Despite a consistent reduction (about 50\%) of training volume, high-intensity training preserves the improvements in aerobic swimming performance and it is more effective on time performance in short distances.

Combined pulmonary fibrosis and emphysema syndrome (CPFE); case report of role of aerobic physical retraining

G. Aquinol, G. Calcagno ${ }^{1}$, A. Bianco ${ }^{1}$, A. Di Sorbo ${ }^{2}$, G. D'Addio', G. De Simone ${ }^{2}$

${ }^{I}$ Department of Medicine and Health Sciences, University of Molise, Italy

${ }^{2}$ CdC Privata di Riabilitazione Villa Margherita, Benevento, Italy

Background: The combination of pulmonary fibrosis and emphysema (CPFE) is a recently defined syndrome. It estimated prevalence is between $5 \%$ and $10 \%$ of cases with diffuse interstitial lung disease. This pathology is present in smokers over 65 years of age and it is frequently under diagnosed. The etiology is yet unknown, but one of the main risk factor remains smoke. In CPFE, upper lobe emphysema and lower lobe fibrosis coexist in a single patient, with a characteristic lung function profile. Apparently, the altered dynamic and static lung 
volume are normally or minimally, however this is in contrast with a significant reduction of carbon monoxide transfer (DLCO) and hypoxemia. The most common symptoms are severe dyspnea, cough, severely impaired transfer capacity for carbon monoxide, hypoxemia at exercise. Moreover, the pulmonary hypertension is a common and important complication in this condition.

Aim: To evaluate the positive effects of aerobic physical retraining in patient with CPFE, in order to consider the physical exercise one treatment option.

Methods: We describe the single case of a 65 years-old male patient with CPFE. Diagnosis was based on chest computer tomography. He complained of dyspnea on exertion, cough and sputum off-white. Pulmonary hypertension (PAP $60 \mathrm{mmHg}$ ) was confirmed by echocardiography. The patient received long term oxygen therapy (OTLT). Retraining program provided 10 to 40 minutes of activity for 2 training sessions per day, for 4 weeks. It consisted of endurance exercise at work rate of $50-60 \%$ of the heart rate and breathing exercises. Before and at the end of program subject was tested with blood gas analysis, spirometry to evaluate respiratory parameters (value-forced expiratory FEV1, forced vital capacity FVC, FEV1/FVC), the walking test $6 \mathrm{~min}$, to assess its physical performance and MRC, VAS and Borg Scales for the evaluation of dyspnea. The quality of life was measured by St. George's Respiratory Questionnaire.

Results: At the end of retraining respiratory parameters did not changes in a patient, but physical performance and quality life and humor improved. Also, the most important result is the reduction of OTLT of about a liter.

Discussion:Functional respiratory parameters do not change but this physical program decreases the perception of dyspnea, the oxygen therapy and improves exercise tolerance in a patient with CFPE, reducing disabilities. This leads to consider the aerobic physical exercise to a therapeutic strategy.

\section{References}

1. Cottin V. and Cordier J. (2009) Fibrosis and emphysema the syndrome of combined pulmonary; Chest : 136 (1): 1-2

2. Kosacka M., Brzecka A., Jankowska R. et al. (2009) Combined pulmonary fibrosis and emphysema: case report and literature Review; Pneumonologia: 77(2): 205-210

3. Nici L., Donner C., Wouters E., Zuwallack R., et al. (2006) American Thoracic Society/European Respiratory Society statement on pulmonary rehabilitation; American Journal of Respiratory And Critical Care Medicine: 173: 1390-1413

\section{Pilates method and multiple sclerosis disease: a pilot study}

\author{
M. Gollin, L. Beratto, C. Mazza \\ Motor Science Research Center, University School of Exercise \& \\ Sport Sciences, University of Turin, Italy
}

Aim: To investigate whether the Pilates method modifies the parameters of flexibility of the trunk, body balance and posture of the column, compared with static stretching exercises in MS subjects.

Methods: Eighteen multiple sclerosis subjects (MS) divided in two groups were studied. The first group (GP) included 8 subjects $(44 \pm 15$ years, $66 \pm 18 \mathrm{~kg}$ weight, $163 \pm 8 \mathrm{~cm}$ height, EDSS $2 \pm 2$ Expanded Disability Status Scale) who followed the Pilates method. The control group (GSS) included 10 subjects ( $45 \pm 9$ years, $62 \pm 13 \mathrm{~kg}$ weight, $163 \pm 6 \mathrm{~cm}$ height, EDSS $4 \pm 2$ ) who did static stretching exercises of a duration of 30 seconds.The groups were evaluated after one month of training (T0) to avoid the learning effect of the exercises, one month later T0 (T1) to observe the effect of one month of training and, three months later T0 (T2), to observe the effect of the long time condition- ing that the Pilates and SS methods have created. The tests used were: 1) Stabilometric platform, to estimate body balance. 2) Sit and reach test, to evaluate trunk flexibility. 3) SpinalMouse ${ }^{\circledR}$, for morphological

evaluation of the spinal column and joint mobility in the sagittal plane using three different positions of the trunk: erect, maximal flexion and extension. 4) Hand grip (HG), to measure forearm strength.

Results: The results showed in GP and GSS a significant variation between T0 vs T2 in the seat and reach test (GSS, Anova p $<0,001$; Post hoc, $\mathrm{p}<0,01,+20 \%$; GP, Anova $\mathrm{p}<0,001$, Post hoc, $\mathrm{p}<0,01,+16 \%$ ). Significant variation was observed in Inc flexion (GSS, $+9 \% ; \mathrm{p}<0,001$ ) and trunk extension $(\mathrm{GP},-13 \% ; \mathrm{p}<0.01)$ measured with the Spinal Mouse. No significant variation was found in $\mathrm{HG}$ and body balance.

Discussion/Conclusion: The Pilates method increased the extension of the vertebral column and pelvis mobility. The SS improved forward column flexion. Training had a conservative effect on strength and body balance. The results indicate that a mix of these types of exercises can be used for MS subjects.

\section{Exercise dependence: a study among nonclinical population}

\section{A. Carraro, I. Ferri, E. Gobbi}

\section{Dipartimento FISPPA, Università di Padova, Italy}

Background: Research documented the benefits of regular exercise (Haskell et al., 2007; United States Department of Health and Human Services, 2008), as well as its role in the prevention and treatment of both physical and mental disorders. On the other hand, exercise may have negative consequences when it becomes excessive. Exercise dependence is a craving for leisure time physical activity, that results in uncontrollable excessive exercise behaviours, manifesting in physiological (e.g. tolerance/withdrawal) and/or psychological (e.g. anxiety, depression) symptoms (Hausenblas \& Downs, 2002a). Exercise dependence can become a compulsive behaviour, where people feel forced to exercise despite injuries, obligations or attempts to reduce their activity (Allegre et al., 2010; Grandi et al., 2010).

The purpose of this cross-sectional study was to explore the prevalence of exercise dependence in a nonclinical mixed gender sample.

Materials and method: A total of 476 participants volunteered, 207 (95 men and 112 women, mean age $=22 \pm 2.2$ years ) were university students, 269 (145 men and 124 women, mean age $=29.8 \pm 11.3$ years $)$ were habitual physical exercisers (organized practice in fitness clubs; $\mathrm{n}=80$ ) or competitive sportsmen/women in endurance (running, swimming, cycling and triathlon; $\mathrm{n}=100$ ) and aesthetic (gymnastic, dance and bodybuilding; $\mathrm{n}=89$ ) disciplines. Participants completed a package of self-report questionnaires including the Baecke habitual physical activity questionnaire - leisure and sport scales - (Baecke, Burema $\&$ Frijters, 1982) and the Exercise Dependence Scale (EDS-21, Hausenblas \& Downs, 2002b).

Results: One-way ANOVA showed significant difference by the students and the sport groups in the Baecke sport scale $(\mathrm{p}<0.001)$, in the EDS tolerance $(p<.001)$, control $(p=0.030)$ and time $(p<0.001)$ subscales and in the EDS total score $(p=0.003)$. Significant differences were found by sport group in the Baecke leisure $(p<0.001)$ and sport $(p<0.001)$ scales, with endurance sportsmen/women reported significantly higher values ( $p<0.001$ at post-hoc test) and in the EDS continuance $(p=0.002)$, tolerance $(p=0.001)$, reduction $(p=0.002)$ and time $(\mathrm{p}=0.002)$ scales.

When considering the whole sample, significant differences by gender $(p<.001)$ were found in the Baecke sport scale (with men had higher values) and in all the EDS scales except reduction, with men had always higher values excluding the withdrawal scale. 
Discussion: Considering the students group, $2.9 \%$ can be categorized as at-risk for exercise dependent, $70.0 \%$ as nondependent-symptomatic and $27.1 \%$ as nondependent-asymptomatic. In the sport group percentage were respectively: $6.3 \%$ at-risk, $78.8 \%$ nondependent-symptomatic and $14.9 \%$ nondependent-asymptomatic. Data appears consistent with prior research findings among habitual exercisers.

Results confirm the importance of recognising exercise dependence among adults engaging in physical activity, to prevent physical and psychological problems.

Movement and cognition: age differences and relations among decision making, motor skills and abilities in childhood

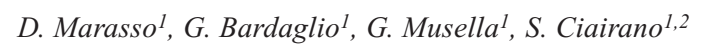

${ }^{1}$ Motor Science Research Center, Research Unit Motor Skills and Development, SUISM, University School of Motor \& Sport Sciences, University of Turin, Italy

${ }^{2}$ Department of Psychology, University of Torino, Torino, Italy

Decision Making (DM) is a vital characteristic of movement mastery. Cognitive and motor systems interact in the process of DM (Johnson, 2009). Even if skilled children show good DM performance since from the age of 8-9 years (William \& Ward, 2003), few studies focused on DM during school age (Marasso, Bardaglio \& Ciairano, under evaluation).

For this reason, our main objective is to study the characteristics of DM in primary school, with a specific attention to the relationship between DM, motor abilities and motor skills.

Participants were 75 children (53\% Males), aged between 8 and 11 years old $(\mathrm{M}=9.7 ; \mathrm{SD}=1.0)$, attending 3rd and 5th classes of primary schools in Cuneo, a city of Piedmont. We used the object control subscale of the Test of Gross Motor Development (TGMD - Ulrich, 1985) to assess skills. About abilities, we assessed the response orientation (Fleishman, 1975) with an ecological test proposed by Hirtz (1985). Finally, to assess DM in team sport, we used a modified version of the GPAI (Oslin, Mitchell, \& Griffin, 1998) for the evaluation of execution, choose, accuracy and speed of DM in a real-game setting. We analyzed control of the ball, and in particular throw and passage, during a session of dodge-ball.

Concerning throw, correlations (divided by class to eliminate the influence of age) showed significant relations between response orientation, skill of control object, speed and accuracy of decision in the 5th class. In the 3 th class, only response orientation was correlated with accuracy. About passage, the percentage of accuracy was related to the percentage of correct execution for both classes.

In light of these results, univariate ANOVA (with gender and BMI as covariates) were used to explore age differences. The two groups differed in the percentage of correct execution when throwing $(\mathrm{F}(1,67)$ $=19.256 ; \mathrm{p}<.001)$, and in percentage of correct chose $(\mathrm{F}(1,67)=$ $11.044 ; \mathrm{p}=.001)$ and accuracy $(\mathrm{F}(1,65)=8.103 ; \mathrm{p}=.006)$, when passing. Also the response orientation was influenced by age.

These preliminary analyses suggest that cognitive and motor systems interact to successful DM in sport. In particular, response orientation is the only stable characteristic between different classes. Differences between results in throw and passage are probably due to the different characteristics of the two actions: level of time constraints and influence of the skill level of the opponent.
Further analysis are need to understand mechanism underlie DM during school age. By this way, instructors and teachers could propose specific interventions to increase the level of DM in sport, and for extension, in all other fields of movement.

\section{Session 11. Physical Activity \& Health - Training \& Performance}

Analysis of weight status and back pain in public employees

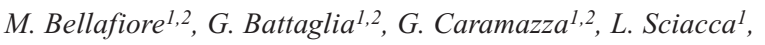
A. Calabretta ${ }^{1}$, A. Bianco ${ }^{1,2}$, A. Palma ${ }^{1,2}$

${ }^{1}$ DISMOT, Palermo, Italy

${ }^{2}$ Regional Sport School of Sicily CONI (Olympic National Italian

Committee), Italy

Introduction: World Health Organization (2007) showed that the job task is responsible for $37 \%$ of low back pain episodes. Among the categories with the highest risk are included the office employees because they often maintain an uncomfortable sitting posture for long time while working.

The aim of our study was to examine the weight status and back pain in office workers.

Methods: One-hundred and twenty workers (29-62 years old) employed to "Corte dei Conti" for Sicily Region with administrative tasks took part into this study and filled a form containing information about their anamnesis, lifestyle and musculo-skeletal disorders. The weight status and intensity of back pain were respectively evaluated with body mass index (BMI) and visual rating scale (VRS) ranging from 'no pain' to 'very strong' (Mannion, 2006). One-way ANOVA test with Bonferroni's correction and Chi-squared test were used to analyze significant differences $(p<0.05)$. Correlations among age, sex, BMI, study title, lifestyle and back pain were calculated.

Results: The sample consisted of men for $39.10 \%$ and women for $60.90 \%$. Men and women had average similar age $(49.09 \pm 6,41 \mathrm{vs}$ $49.46 \pm 6.10$ years old) while were significantly different for weight $(80.42 \pm 8.03 v s$

$64.4 \pm 11.50 \mathrm{~kg})$ and height $(176.42 \pm 6.04 v s 162.16 \pm 6.04 \mathrm{~cm})$. BMI indicated that the weight gain was bigger in men than women $(55 \% \mathrm{vs}$ $44 \%$ ). This result was significantly related to a lower study title in women but not in men. Additionally, in both sex an active lifestyle was associated with a decrease in obese employees but with no variation in overweight status. The women reported stronger pain in cervical than lumbar spine. The opposite condition has been stated by men. A more intense pain was correlated to sedentary lifestyle and overweight status in both sex.

Discussion: These results indicate that a large number of employees shows a weight gain and back pain. Therefore, the organization of education programs about nutritional and postural habits by managers might be a useful strategy for improving the productivity and life quality of their workers.

References

- Mannion AF et al. (2006) Development of a German version of the Oswestry Disability Index. Part 1: cross cultural adaptation, reliability, and validity, Eur Spine, 15: 55-65 
Sports practice and personality assessment in adolescents exposed to the 2009 earthquake in L'Aquila, Italy: a longitudinal study

\section{Valenti, F. Masedu, M.G. Vinciguerra}

Dept. of Biotechnological and Applied Clinical Sciences, University of L'Aquila, L'Aquila, Italy

Objective: To assess and estimate the personality changes that occurred before and after the 2009 earthquake in L'Aquila and to model the ways that the earthquake affected adolescents according to gender and sport practice. The consequences of earthquakes on psychological health are long-lasting for portions of the population, depending on age, gender, social conditions and individual experiences. Sports activities are considered a factor with which to test the overall earthquake impact on individual and social psychological changes in adolescents.

Design: Before \& after design.

Setting: Population-based study conducted in L'Aquila, Italy, before and after the 2009 earthquake.

Participants: Before the earthquake, a random sample of 179 adolescent subjects who either practised or did not practise sports (71 vs 108 respectively). After the earthquake, of the original 179 subjects, 149 were assessed a second time.

Primary outcome measure: Minnesota Multiphasic Personality Inventory for Adolescents (MMPI-A) questionnaire scores, in a supervised environment.

Results: An unbalanced split plot design, at a 0.05 significance level, was carried out using a linear mixed model with quake, sex and sports practice as predictive factors. Although the overall scores indicated no deviant behaviours in the adolescents tested, changes were detected in many individual content scale scores, including depression (A-dep score Mean \pm SEM: before quake $=47.54 \pm 0.73$; after quake $=52.67 \pm 0.86$ ) and social discomfort (A-sod score Mean \pm SEM: before quake $=49.91 \pm 0.65$; after quake $=51.72 \pm 0.81$ ). The MMPI-A profiles show different impacts of the earthquake on adolescents according to gender and sport practice.

Conclusions: The differences detected in MMPI_A scores raise issues about social policies required to address the psychological changes in adolescents. The current study supports the idea that sport should be considered part of a coping strategy to assist adolescents in dealing with the psychological effects of the earthquakes on their personalities.

\section{Reliability and validity of dexterity circuits in the "Alfabetizzazione Motoria" project}

\author{
F. Merni ${ }^{1,3}$, C. Aversa ${ }^{3}$, S. Brugnara ${ }^{3}$, A. Ceciliani $^{2,3}$
}

${ }^{1}$ Dipartimento di Istologia, Embriologia e Biologia Applicata, Università di Bologna, Bologna, Italy

${ }^{2}$ Dipartimento di Scienze dell' Educazione "Giovanni Maria Bertin", Università Di Bologna, Bologna, Italy

${ }^{3}$ Facoltà di Scienze Motorie, Università di Bologna,Bologna, Italy

"Alfabetizzazione Motoria" is a national project involving primary school students, carried out jointly by the Ministry of Education Universities and Research and the Italian Olympic Committee, with the purpose of promoting and transmitting the importance of practicing sport. The project involves a monitoring program based on 5 dexterity circuits, one for each primary school class. The purpose of this study was to assess the reliability and validity of such dexterity circuits. Data were collected in March (T1) and in May (T2) in two primary schools:
Casalecchio Di Reno (10 classes, 178 children), and Zola Predosa (6 classes, 135 children). In the second school, a test-retest procedure was carried out both in at $\mathrm{T} 1$ and $\mathrm{T} 2$, with the circuits repeated two times in the same day. The subjects were filmed while executing the circuit, and an a-posteriori evaluation was carried out concerning the total time required to perform the circuit, the time needed to execute different motor tasks included in the circuit, and the number of errors in each section. Analysis of variance and Cronbach's Alpha were used to assess the reliability. Principal component analysis (PCA) and hierarchical cluster analysis were used to analyze the internal consistency of each circuit.

The Alpha coefficient ranged from 0.6 and 0.9 in the different trials of the circuits, showing a sufficient to good reliability for all the classes. However, the differences between trials were always statistically significant, even when the tests were repeated two times in the same session. This leads to hypothesize the presence of a learning effect involving short-term improvements. This hypothesis is confirmed especially in the fifth class, in which the improvement throughout the four trials is much more evident than in the other classes. The multivariate statistical analysis allowed to evaluate which sections of the circuits provided the most relevant contribution to the total time. The balance, slalom and hurdles sections were the most related with the overall performance. This was evident in all the classes with the exception of the fourth. In all the circuits, the highest difficulties were noticed in the balance and hurdles sections that strongly affected the total time. This was confirmed by the analysis of errors, actually showing the highest number in those sections. Four error types only concerning the balance section negatively affected the performance. In the other cases it would be worth to insert a time penalty because making an error allows to gain time, as shown in the balance section of second classes both in March and May. Factor analyses showed that the balance sections are the most important in determining the final result both in a positive and negative direction. This kind of studies allows to perform more accurate and valid assessments.

Specific performance models evidenced in elite taekwondo athletes based on different body protector systems

F. Tornello ${ }^{1,2,3}$, L. Capranica $^{\text {I }}$, C. Minganti ${ }^{2}$, S. Chiodo ${ }^{1,3}$, A. Tessitore 1

${ }^{I}$ DISMUS, University of Rome Foro Italico, Rome, Italy

${ }^{2}$ University of Magna Graecia, Catanzaro, Italy

${ }^{3}$ Italian Taekwondo Federation, Italy

Taekwondo is a full-contact sport in which both head and body protectors are used. After becoming an official Olympic sport at the 2000 Sydney Games, there has been the necessity of rendering it more spectacular and of having an objective scoring system. To this end were the changes of the "Competition Rules" and the "electronic Protector and Scoring Systems (PSS) introduced. Currently the "Official Competition Rules" permit the athletes to compete both with trunk protectors not equipped with electronic sensors and with the PSS.

Purpose: Thus, this study was aimed at investigating whether different trunk protector systems and types of scoring affect the performance of elite taekwondo athletes.

Methods: Thirty six official matches of elite male senior athletes, twelve for each trunk protector system (Traditional-TRA, Daedo-DAE and LaJust-LAJ), were observed during three different International Taekwondo Championshi! ps. The technical-tactical analysis was applied to investigate: a) The number of combat activity profiles in relation to Fight (F), Non-Fight (NF) and Stoppage (ST) (Tornello et al, in press); b) The strategy adopted for actions (Attack, Defense and Block 
actions); c) The selected scoring area of the kicks was classified into trunk and head (Falco et al, 2012).

Statistical analysis: Means, standard deviations and ranges were calculated for each dependent variable. Statistical analyses were conducted using an SPSS package (version 17) and the criterion for significance was set at $\mathrm{P} \leq 0.05$. The effects of the different protector systems on each technical and tactical indicator were identified using chisquare tests. Successively, for every significant effect evidenced, a post-hoc analysis was applied on each comparable couple of competition levels by means of a chi-square to which the Bonferroni correction was applied.

Results: The tru! nk protector systems exerted a notable influence on all considered tec hnical-tactical indicators: Activity profiles, Strategy and Selected scoring area $(\mathrm{P}<0,001)$. Post hoc revealed: For activity profiles a significant difference of DAE [54 $\pm 19(33-83)] v s$ LAJ [62 $\pm 20(36-107)]$ and TRA [61 $\pm 25(30-101)]$ for $\mathrm{F}$ and between all protector systems, DAE $[10 \pm 6(2-21)]$, LAJ $[9 \pm 4(5-19)]$ and TRA $[7 \pm 4(2-$ 12)], for ST; Differences for Strategy in Attack between DAE [29 $\pm 12(11-59)]$ and LAJ [33 $\pm 13(15-67)]$, and between DAE [16 $\pm 8(7-$ 38)], LAJ [12 $\pm 6(3-25)]$,TRA [11 $\pm 8(2-32)]$, and, between DAE [10 \pm 7(2-28)], LAJ [17 $\pm 9(3-38)]$, TRA [17 $\pm 12(3-56)]$, for Defense and Block actions respectively; A significant preference of kicking in DAE [32 $\pm 16(12-62)]$ vs LAJ [29 $\pm 12(11-55)]$ and TRA [27 $\pm 12(9-49)]$ to the trunk, and between all protector systems, DAE [6 $6 \pm 3(2-11)]$, LAJ $[11 \pm 7(1-25)]$ and TRA $[9 \pm 8(1-38)]$, to the head.

Conclusions: The findings of this study showed that each protector system imposed a specific performance model of combat. This fact suggests that athletes have to adopt specific strategies in relation to the typology of protector system they will use during competition.

Reference:

- Falco et al (2012), Tornello et al, in press.

\section{Stress-recovery balance in road cyclists during the "Girobio-Giro} d'Italia Elite \& Under23"

\section{F. Forzini ${ }^{1}$, T. Agostini ${ }^{1}$, L. Bortoli ${ }^{2,3}$, S. Di Fronso, ${ }^{2,3}$, C.Robazza ${ }^{2,3}$, M. Bertollo ${ }^{2,3}$}

${ }^{1}$ Dept Life Sciences, Trieste University, Trieste, Italy

${ }^{2}$ Behavioral Imaging and Neural Dynamics (BIND) Center,

D’Annunzio Univ., Chieti, Italy

${ }^{3}$ Dept Medicine and Aging Sciences, D'Annunzio University, Chieti, Italy

Aim: The purpose was to investigate the influence of nine-stage bicycle competition on stress recovery balance in elite road cyclists. In athletes the accumulation of stressors, accompanied by insufficient recovery, causes a change in homeostasis of the psycho-physical general well-being which can lead to dysfunctional phenomena of overreaching or overtraining, (Jones \& Tenembaum, 2009). There is a doseresponse relationship between training loads and stress-recovery balance, as assessed by the multidimensional Recovery-Stress Questionnaire for Athletes (RESTQ-Sport, Kellmann \& Gunther, 2000). Given that RESTQ-Sport also includes measures of other forms of stress and recovery responses that are not necessarily related to training overload per se, competition may also potentially cause a mismatch between stress and recovery (Kellmann, 2002).

Methods: Participants were 72 road cyclists aged $19-26$ years $(\mathrm{M}=$ $21.9, \mathrm{SD}=1,6)$ involved in the "Giro d'Italia elite \& under 23". The Italian version of RESTQ-Sport (Kellmann \& Kallus, 2001) was administered during the day before of the first stage and repeated again before the last stage.

Results: RM-MANOVA showed a within subjects mean effect on time
(Wilks's lambda $=3.44 \mathrm{~F}(19,53)=5.32, \mathrm{p}<0.001$, eta $2=0.656$, power=1.00). Following univariate test showed differences in 11 of 19 subscales comparing pre and post competition, with no differences in the last 4 sport-specific recovery scales (Being in Shape, Personal Accomplishment, Self-Efficacy, Self-Regulation): General Stress $\mathrm{F}(1,71)=31.18, \mathrm{p}<0.001$; Emotional Stress, $\mathrm{F}(1,71)=19.65, \mathrm{p}<0.001$; Social Stress $\mathrm{F}(1,71)=23.20, \mathrm{p}<0.001$; Fatigue, $\mathrm{F}(1,71)=59.13$, $\mathrm{p}<0.001$; Lack of Energy $\mathrm{F}(1,71)=12.97, \mathrm{p}<0.001$; Physical Complaints, $\mathrm{F}(1,71)=45.27, \mathrm{p}<0.001$; Physical Recovery, $\mathrm{F}(1,71)=10.25, \mathrm{p}=0.002$; General Well-being, $\mathrm{F}(1,71)=9.48$, $\mathrm{p}=0.003$; Disturbed Breaks, $\mathrm{F}(1,71)=34.25, \mathrm{p}<0.001$; Emotional Exhaustion, $\mathrm{F}(1,71)=37.82, \mathrm{p}<0.001$; Injury, $\mathrm{F}(1,71)=22.90, \mathrm{p}<0.001$ ! .

Conclusions: Findings confirm that RESTQ-Sport is a sensitive instruments to measure stress recovery balance in athletes and suggest that hard competition, like a bicycle race stage, have strong impact on stress recovery balance, affecting general stress and recovery, specific stress but not specific recovery that possibly help the athletes to cope with the race.

References

- Jones, C. M., Tenenbaum, G. (2009) Adjustment disorder: A new way of conceptualizing the overtraining syndrome. International Review of Sport and Exercise Psychology, 2: 181-197

- Kellmann, M. (Ed.) (2002) Enhancing recovery: Preventing underperformance in athletes. Champaign, IL: Human Kinetics

- Kellmann, M., Kallus, K. D. (2001) Recovery-Stress Questionnaire for athletes: User's manual. Champaign, IL: Human Kinetics

- Kellmann, M., Günther, K. D. (2000) Changes in stress and recovery in elite rowers during preparation for the Olympic games. Medicine and Science in Sports and Exercise 32: 676-683

Putting in golf: a comparison between professional and amateur players based on stabilometric analysis

\section{Pizzigalli ${ }^{1,2}$, F. Gennaro $^{2}$, G. Nazzi ${ }^{2}$, A. Rainoldi $^{1,2}$}

${ }^{1}$ Centro Ricerche Scienze Motorie, Scuola Universitaria Interfacoltà in Scienze Motorie, Università degli Studi di Torino, Italia

${ }^{2}$ Scuola Universitaria Interfacoltà in Scienze Motorie, Università degli Studi di Torino, Italia

Putting is one of the most important golf shots both for professional and amateur players; the literature shown that no score differences among them in short distance $(<3.5$ meters). Therefore, the aim of this study was to investigate the biomechanical aspects of the putting comparing five professional and five amateur players performing five putting swings, at 3.5 meters of distance. The biomechanics of the movement was recorded by two digital cameras $(50 \mathrm{~Hz})$ and divided into four phases: from one second before the back swing to the back swing; from the back swing to the inversion; from the inversion to the impact and from the impact to one second after the impact. The analysis of the distribution of the plantar loads was acquired by four load cells: (FA6 anterior right side; FA4 anterior left side; FA8 posterior right side and FA2 posterior left side) housed in a stabilometric board (TecnoBody PROKIN PK 214 P). Besides, the analysis of trunk's inclinations was acqu! ired by a sternal accelerometer (Tecnobody Prokin Trunk Sensor TKS ).

In the first phase, professionals exerted a lower plantar pressure on FA4 than amateurs $(\mathrm{p}=0.008)$. Also $1 \mathrm{~s}$ before the backswing and during the backswing, professionals exerted a lower plantar pressure than amateurs on FA6 ( $p=0.0015 ; \mathrm{p}=0.0001$ respectively). Moreover, analyzing trunk inclinations, professionals remained on the vertical $(p=0.014)$ and tilted the trunk of $4^{\circ}$ to the left, while the amateurs only $2^{\circ}$ $(p=0.016)$. During the second phase, professionals did not increase 
plantar pressure, whereas amateurs did it, on FA2 $(\mathrm{p}=0.0022)$ and tilted the trunk $13^{\circ}$ to the right side $(\mathrm{p}=0.0001)$ such as amateurs. During the inversion phase amateurs showed a larger FA6 plantar pressure $(\mathrm{p}=0.0165)$ and a lower trunk tilt to the right side $(\mathrm{p}=0.0001)$ compared to professionals. During the third phase professionals continued their tilt trunk on the right side greater $(\mathrm{p}=0.008)$ than amateurs such as during the impact phase $(\mathrm{p}=0.0001) 13^{\circ}$ and $2^{\circ}$ respectively. During the! last phase professionals exerted less plantar pressure than amateurs on
FA4 $(p=0.0468)$. One second after the ball impact, professionals continued their trunk tilt to the right side more than amateurs $(p=0.0001)$. In conclusion, findings highlight a better balance distribution of the plantar loads and a stronger trunk counterbalance of the putter in professionals group. Moreover, analyzing the five putts of the two groups of players, professionals demonstrated to be more constant in the putting execution, highlighting a better repeatability of the technical movement. 
Adami P. E. 51

Agnello S. 5

Agostini T.69

Alberti G. 8

Alesi M. 3,21,22,24,25,64

Alfieri A. 5,63

Ammendolia A. 41

Amodio E. 46

Andolina G. 11

Andreoli P. 40

Annino G. 16,28,40

Aprile A. 42

Aquino G. 10,64-65

Arrigo R. 4

Asciutto R. 46

Aversa C.68

Bacchi E. 11,15

Baldari C. 6

Baraldo A. 53

Bardaglio G. 16,20,67

Barresi M. 10

Bartolucci C. 14

Bascelli A. 11

Battaglia C. 4

Battaglia G. 20-22,41-43,64,67

Bazzucchi I. 6

Beato M. 43,61

Bellafiore M. 20,41-43,64,67

Bellagamba S. 14

Bellavia D. 29

Bellia A. 40

Bellian A. 16

Bellistri G.65

Beratto L. 18,66

Berlutti G. 14

Berlutti G. 14

Bernardi M. 51

Bertollo M. 1,45,69

Bianco A. 12,18-20,24,41-43,45,47,53, 64,67

Bianco A. 65

Biscarini A. 23

Blando F. 55

Boccia G. 56

Bonato M. 13,33,65
Bonora E. 11

Bonsignore M. R. 4

Borgoni A. 56

Borrione P. 4

Bortolan L. 8,35,43,57,61-62

Bortoli L.1,45,69

Bossolasco S. 13

Botti F. M. 23

Bovino N. 58

Branca J. J. V.60

Brasili P. 31

Brighenti A. 5,15,35

Brighina F. 53

Brignardello G. 43,61

Brugnara S.68

Brunetti A. 23

Brunetti O. 23

Brustio P. 39

Cairo F. 30

Calabretta A.67

Calamusa G. 46

Calavalle A. R. 11

Calcagno G. 4,10,64,65

Camomilla V. 32

Campanozzi A. 1

Cao G. 40

Capelli G. 4

Capogrosso M. 19

Capranica L. 17,53,55,68

Caramazza G. 42,47,64,67

Carandente F. 8

Cardillo I. 4

Carello C. 27,34

Carnicelli V. 22

Carraro A. 25-26,50,63,66

Casagrande A. 3

Castagna S. 12

Cataldo A. 29

Cataldo M. C. 41

Cavedon V. 15,29,58

Cè E. 5,57

Ceciliani A.68

Cerasola D. 29

Cerrotta M. 33

Cevese A. 53
Chemello A. 9

Chiavaroli S.60

Chiodo S.68

Ciairano S. 16,20,67

Cibelli C. 17

Cilia S. 7,24

Cincimino S. 54

Cinque P. 13

Ciuffarella A. 58

Collella D. 1,16,39

Collier S. R. 6,52

Comotto S.61

Condello G. 8

Contrò V. 19

Cook C. 6

Coratella G. 9,28,43,47

Corradini G. 43

Corsello G. 47

Cortis C. 13,53,55

Costantino C. 46

Costantino D. 20,21

Costantino S. 23

Curry C.D. 6,52

D'Addio G.65

D'Aliesio F. 4,51

D'Amico M. A. 11

D'Angelo C. 47

D'Arcangelo G. 18

D'Egidio R. 22

D'Ottavio S. 16,28,33

Dal Toè L. 18

Dalla Libera M. 28

Davanzo M. 17

De Rosas M. 17

De Simone G.65

De Vita F. 43,61

Delia G. 45

Dell'Aia M. 15

Di Angelo L. 12

Di Baldassarre A. 11

Di Cagno A. 4,30,64

Di Fabio A.60

Di Fonso A. 11,69

Di Fronso S. 44

Di Giminiani R. 38 
Di Giulio A. 12

Di Giulio A. 2,38

Di Giuseppe F. 8

Di Gregorio M. N. 2

Di Michele R. 1

Di Muzio D.61

Di Pasquale M. 46

Di Sarra D. 15

Di Sorbo A.65

Di Stefano P. 12

Di Stefano S. 7,24

Di Tore P. A. 9,22,29,32,33

Di Trapani S. 47

Digennaro S. 48

Digennaro S. 56

Doria C. 43

Esposito F. 5,57

Fanò-Illic G. 6,43

Fasano M. 27

Fattorini L. 51

Faure Rolland F. A. 30

Federici A. 14,39

Federico B. 4

Feletti L. 36

Felici F. 6

Ferreri L. 36

Ferretti G. 19

Ferri I. 25-26,50,63,66

Fierro B. 53

Filingeri D. 18,45,52

Fiorellini A. 26

Fiorilli G. 4,64

Fischetti F. 35,46

Formenti D. 8

Formicola D. 36,37

Forzini F.69

Foti C. 40

Francavilla C. 37

Frank C. 18

Frau M. 40

Friard O. 30

Fulle S. 6

Galardini A. 26

Galbusera C. 3,9

Gallà M. A. 20

Galli L. 13

Gallina S. 11

Gallone D. 35

Gennaro G.69

Gerrett N. 52

Gervasi M. 11
Gerzevic M. 10,32

Giachetti A. 58

Giacobini M. 36

Giglia G. 53

Giombini A. 4,64

Gobbi E. 25,26,50,63,66

Gollin M. 18,36,66

Gollin M. 31

Grainer A. 43

Granatelli G. 33

Greco M. 39

Guadagna P. 47

Guastamacchia R. 46

Gueli I. 53

Guerra A. 31

Guerra E. 34

Guidetti L. 6

Guidotti F. 55

Gulisano M.60

Havenith G. 51, 52

Hodder S. 52

Iellamo F. 40

Impedovo P. 55

Impellizzeri F. 9

Imperlini E. 5,63

Inguglia M.64

Innocenti G.60

Inzitari M. T. 12

Iona T. 12,41

Iuliano E. 10,64

Ivaldi M. 36,37

Izzicupo P. 11,22

James M. L. 52

Jemni M. 45

Jia W. 6

Krustrup P. 5

La Delfa G. 24,37,62

La Spina A. 56

La Torre A. 10,13,32,65

La Torre R. 28

Lacitignola D. 13

Landram M J. 52

Lanza M.2,11,44,47

Lanzano R. 51

Lanzerotti R. 58

Laricchia R. 46

Lebone P. 16

Leonardi A. 43

Leone L. 49,63

Limonata E. 5,57

Lipoma M. 7,10,41,58,62
Liubicich M. 17,39,40

Lo Curcio F. 42

Loddo A. 40

Lombardo M. 16,28,40

Longo D. 39

Loria S. 34

Lovato C. 58

Lucertini F. 14,39

Maggio M. C 47

Maggioni M.65

Magistro D. 17,39,40

Magno F. 16,20

Mammina C. 41,42,45,46

Mancini A. 5,63

Mancuso E. 45

Mango P. 10,37,41,58,62

Manno R. 16

Manzi V. 40

Marandola D. 4

Marasso D. 16,20,67

Marcello F. 40

Maringhini G. 46

Martelli S. 50

Martini S. 34

Martone D. 5

Marzorati M.65

Masedu F. 38,68

Mazza C.66

McAnulty S. R. 52

Melchiorri G. 16,18

Merati G. 13

Merni F. 1,68

Messina R. 8

Messina S. 43

Meucci M. 6

Micheletti Cremasco M. 14,30

Milanese C. 15,29,58

Minganti C. 55,68

Modena R. 5,15,35

Moghetti P. 11,15

Moisio V. 2

Mondelli I. 26

Mondini P. 13

Mondonico M. 34

Montaruli A. 17

Montini M. 6

Morano M. 1,39

Moretti B. 35,46

Moretti L. 35

Morici G. 4

Morici M. 46 
Moro T. 12,45

Morucci G.60

Mulasso A. 17,39,40

Musella G. 16,67

Nadalet L.65

Napoli G. 46

Napolitano S. 9,32,33

Nart A. 59

Nazzi G.69

Negri C. 11,15

Nichino P. 34

Nicolosi S. 7,24

Nonis D. 10,32,65

Notarnicola A. 35,46

Novero F. 17

Omeri M. 9

Orrù $\mathrm{S} .63$

Pacini S.60

Padua E. 16,18,28,40

Padulo J. 10,28,59

Palazzo F. 28

Palma A. 18-20,24,41-43,45,47,53, 64,67

Palma R. 20,42

Palmeri A. 46

Pannella A. 48,50

Panzarino M. 16

Paolo A. 12,18

Parisi A.65

Parisi L. 21, 22

Parisi S. 46

Patti A. 41,47

Pavei G. 13

Pellegrini B. 5,8,15,35,57,62

Pepi A. 3,24, 25

Perini R. 19

Perrotta F. 48,50

Pesce C. 49,53,63

Petrollini G. 34

Petrucci M. 43

Piacentini M. F. 55,61

Piazza M. 14

Piazza M. 4,30

Piccinino A. 16

Picerno P.61

Pietrangelo T. 6

Pignato S. 24,37,41,58,62

Pigozzi F. 4,65

Pilloni M. 34

Pillosu P. 40

Piscitelli F. 29,58
Pizzigalli L. 36,69,59

Pizzolato F. 59

Pomara F. 43

Porcelli S.65

Porro N. 49

Posenato L.61

Procenesi M. 23

Proia P. 19,42

Pugliese L. 10,65

Quagliani K. 14

Quarantelli M. 3,9

Quinzi F. 32

Quirino Lucciola A. 13

Rabaglietti E. 16,20

Raccuglia M. 18,24,41,42,45

Raimondi P. 12

Rainoldi A. 36,37,56,69

Raiola G. 9,22,29,32,33

Rampichini S. 5,57

Randers M. B. 5

Rappo G. 25

Rastelli F.60

Redortier B. 52

Reggiani C. 43

Rigazio S. 54

Rinaldi M. 14,39

Rinaldo N. 43,47

Robazza C. 1,45,69

Roccella M. 21, 22

Rocchi M. B. L. 11

Rodio A. 13,51

Rodorigo M. 2

Roggero A. 40

Romanazzi M. 56

Roppolo M. 17, 18,39,40

Roselli M. I. 17

Rossetta A. 6

Rossi A. 47

Rossi G. 22

Ruscello B. 16,33

Russo G. 29,30

Russo L. 30,34,58

Rutigliano I. 1

Sacchetti M. 6

Saladino P. 19

Salli M. 40

Salvadori I. 44

Santoro L. 54

Savoldelli A. 5,15, 35,43,62

Sbraccia P. 40

Sbriccoli P. 32
Scaglione A. 56

Scagliotti C. 18

Scarafiotti E. 36

Scarpa S. 59

Scatigna M. 2,22

Schembri R. 7,24,41

Schena F. 2,5,8,9,15,28,35,43,47, $57,61,62$

Schiera G. 19

Schumacher Y. O. 65

Sciacca L.67

Scichilone N. 4

Scotton C. 59

Semprini G. 1

Sgrò F. 10,37,58,62

Siragusa P. 43

Sisti D. 11

Sivieri A. 19

Spaziani S.63

Spiga F. 31

Spina S. 24

Spineto C. 11

Squeo M. R. 51

Stocchi V. 11

Tabacchi G. 45, 46

Tafuri S. 35,46

Tallaria L. 48

Tancredi V. 16,18,28

Tarperi C. 53,61

Tessitore A. 8,55,68

Testa D. 21, 22

Thomas E. 18,45

Tihanyi J. 38

Tinto A. 14,27,30,34

Tomasoni E. 56

Tornello F.68

Toselli S. 31

Tosi F. 15

Traina M. 19,29

Trevisson P. 30

Triggiani A. I. 17

Triossi T. 18

Trobia G. 46

Trombetta M. 11

Tursi D. 9

Turturici M. 36

Utter A. C. 52

Valenti M. 38,68

Valenzano A. 17

Vando S. 28,59

Vannini E. 56 
Varalda C.61

Vasile Simone S. 45

Verdecchia L. 28

Vernillo G. 43

Verratti V. 43

Vettorello N.65

Vicenti G. 46
Villani S. 17

Vincenzo B.59

Vinciguerra M. G. 2,2,22,60,68

Vitale J. 8

Welsh J. L. 52

Weydahl A. 8

Zambotti F. 15
Zamparo P. 8

Zancanaro C. 15,29,58

Zangla D. 4,29,53

Zanolin M. E. 11

Zoffoli L. 39

Zoppirolli C. 8,57,62

Zucchetti G. 20 
$\underline{1^{\text {st }} \text { National Congress SISMES }}$

Date: October $2^{\text {nd }}-3^{\text {rd }}, 2009$

Venue: Noto Marina, Sicily

Abstracts: 85

Participants: 157
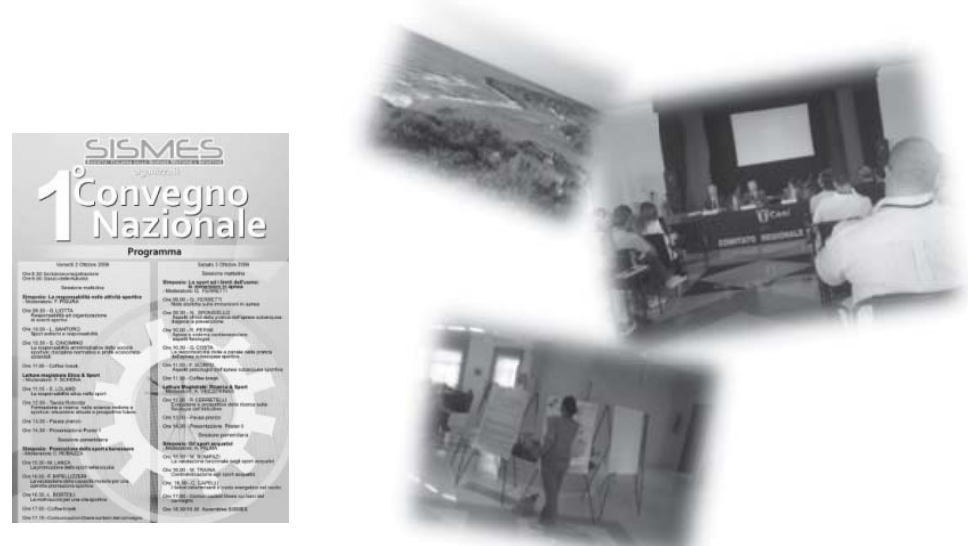

$\underline{2^{\text {nd }} \text { National Congress SISMES }}$

Date: September $30^{\text {th }}-$ October $2^{\text {nd }}, 2010$
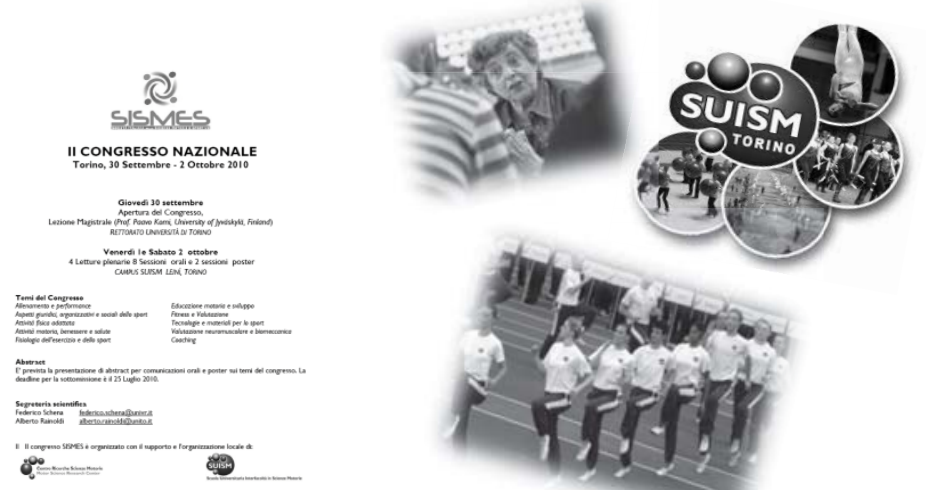

Participants: $\mathbf{2 3 0}$

$3^{\text {rd }}$ National Congress SISMES

Date: September $29^{\text {th }}$ - October $1^{\text {st }}, 2011$

Venue: Verona, Veneto

Abstracts: 164

Participants: 306
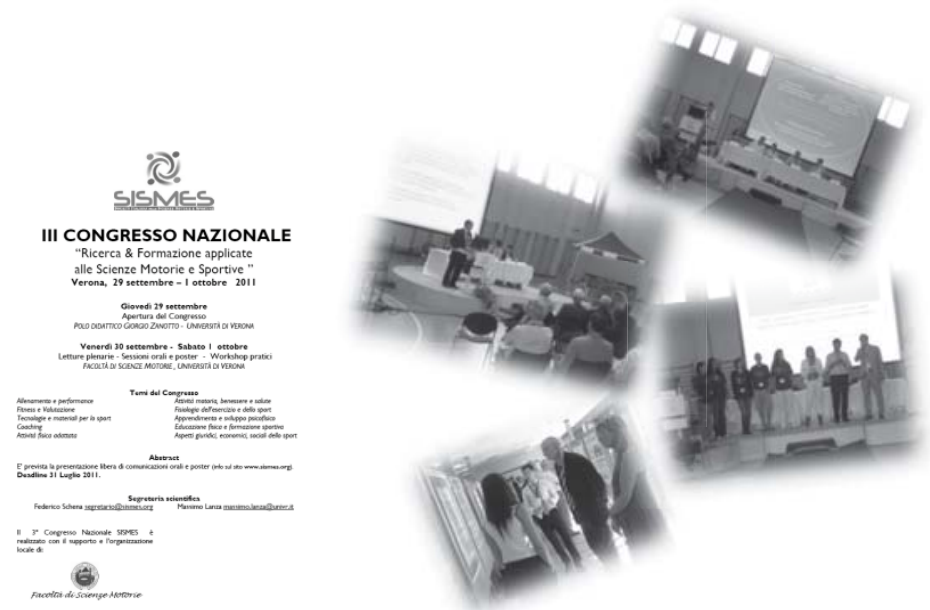
$4^{\text {th }}$ National Congress SISMES

Date: October $5^{\text {th }}-7^{\text {th }}, 2012$

Venue: Palermo, Sicily

Abstracts: 172

Expected participants: 350
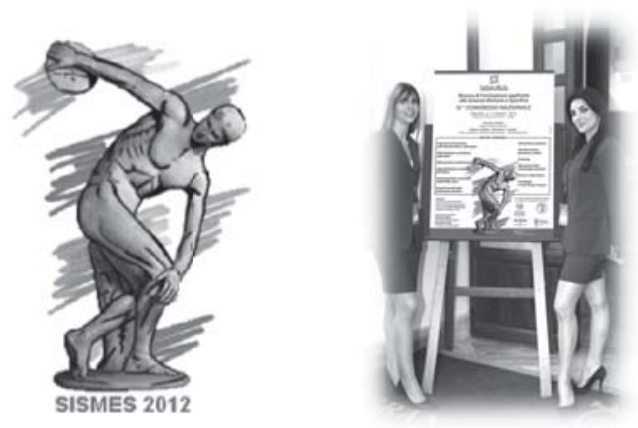

Abstract distribution by Author's affiliation

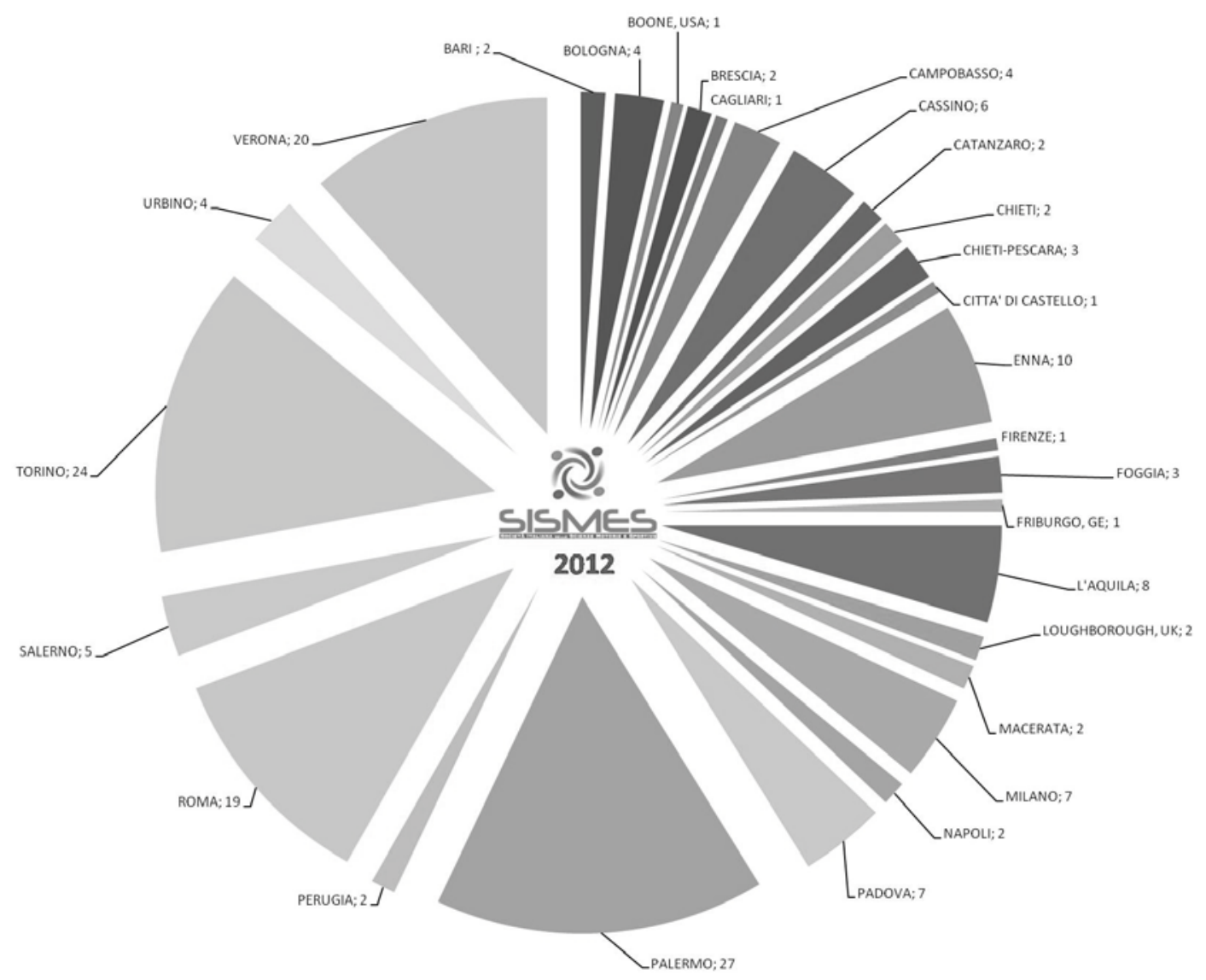

